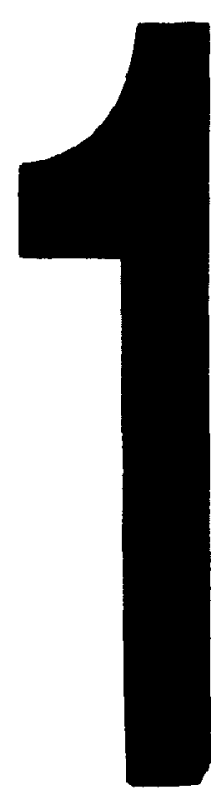

PM-1 3\%" $x 4$ " PHOTOGRAPHIC MICROCOPY TARGET NaS 1010? ANSI/ISO \#2 EQUIVALENT

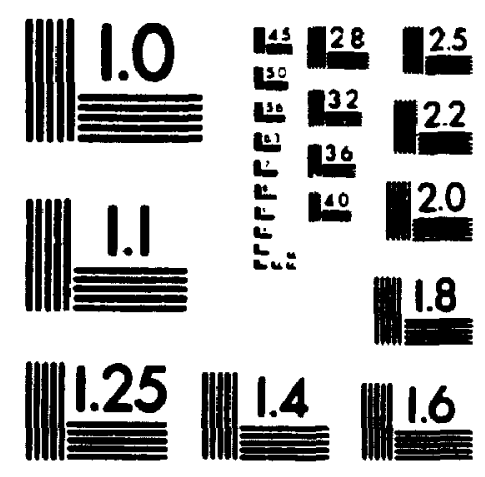

PRECISIONEW RESOLUTION TARGETS 
National Library

of Canada

Acquisitions and

Bibliographic Services Branch

395 Wellington Sireet

Ottawa. Ontario

KIA ONA
Bibliothéque nationale

du Canada

Direction des acquisitions et des senvices bibliographiques

395. rue Wellington

Otawa (Ontario)

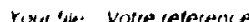

\section{NOTICE}

AVIS

The quality of this microform is heavily dependent upon the quality of the original thesis submitted for microfilming. Every effort has been made to ensure the highest quality of reproduction possible.

If pages are missing, contact the university which granted the degree.

Some pages may have indistinct print especially if the original pages were typed with a poor typewriter ribbon or if the university sent us an inferior photocopy.
La qualité de cette microforme dépend grandement de la qualité de la these soumise au microfilmage. Nous avons tout fait pour assurer une qualité supérieure de reproduction.

S'il manque des pages, veuillez communiquer avec l'université qui a conféré le grade.

La qualité d'impression de certaines pages peut laisser à désirer, surtout si les pages originales ont été dactylographiées à l'aide d'un ruban usé ou si l'université nous a fait parvenir une photocopie de qualité inférieure.

La reproduction, même partielle, de cette microforme est soumise à la Loi canadienne sur le droit d'auteur, SRC 1970, c. C-30, et ses amendements subséquents. 


\title{
Measurement and Performance Analysis of World Wide Web Applications
}

by

\author{
Jie Ma
}

\author{
A thesis submitted to \\ The Faculty of Graduate Studies and Research \\ in partial fulfillment of the requirements for the degree of \\ Master of Engineering \\ Faculty of Engineering \\ Department of Systems and Computer Engineering \\ Carleton University, \\ Ottawa, Ontario, Canada
}

January 19, 1996

Supervisor: Professor C. M. Woodside

(C) copyright

1996, Jie Ma 
National Library

of Canada

Acquisitions and

Bibliographic Services Branch

395 Werington Streed

Onawe. Onterio

K1A ONA
Biblotheque nationale

du Canada

Direction des acquisitions et

des senices bibliographiques

395. rue Wellington

Orima (Ontaro)

K1A ONA

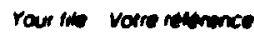

Or ine notre reldrence
The author has granted an irrevocable non-exclusive licence allowing the National Library of Canada to reproduce, loan, distribute or sell copies of his/her thesis by any means and in any form or format, making this thesis available to interested persons.
L'auteur a accordé une licence irrévocable et non exclusive permettant à la Bibliotheque nationale du Canada de reproduire, prêter, distribuer ou vendre des copies de sa thèse de quelque manière et sous quelque forme que ce soit pour mettre des exemplaires de cotte thèse à la disposition des personnes intéressées.

L'auteur conserve la propriété du droit d'auteur qui protège sa thèse. Ni la thèse ni des extraits substantiels de celle-ci ne doivent être imprimés ou autrement reproduits sans son autorisation.

ISBN $\quad 0-612-08980-0$ 


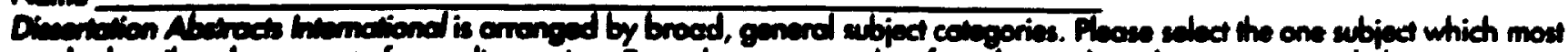

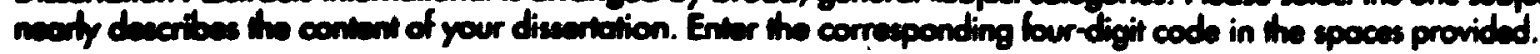

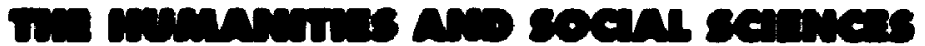
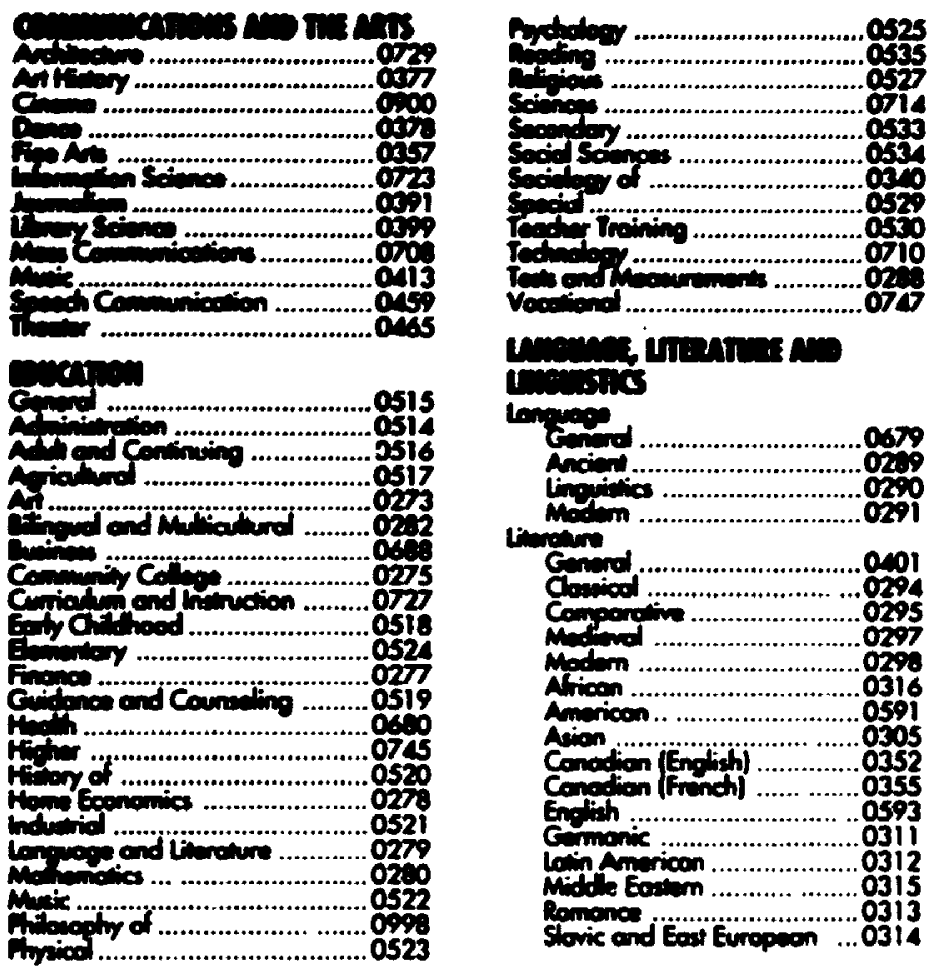

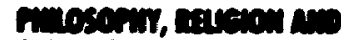
ingeor

momophy .............................0422

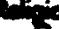

Extrod .........................0318

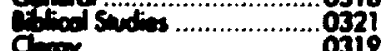

QDery.

nitom of .......................0322

Thedegy ..................................0868

sents:iss

Arution sudie

Arimesing:

Adincion ................032

Cingra..........................0326

hiprice

Genced ............................0310

focounting ............................. oz

gonding

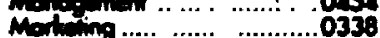

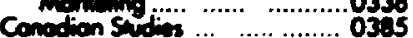

Economics

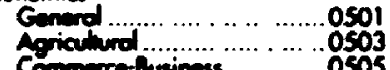

finconese

lowery

Thery

Fentore

Gogreciny

Hivery

Generot

0510

Osil

0358

0351

0578

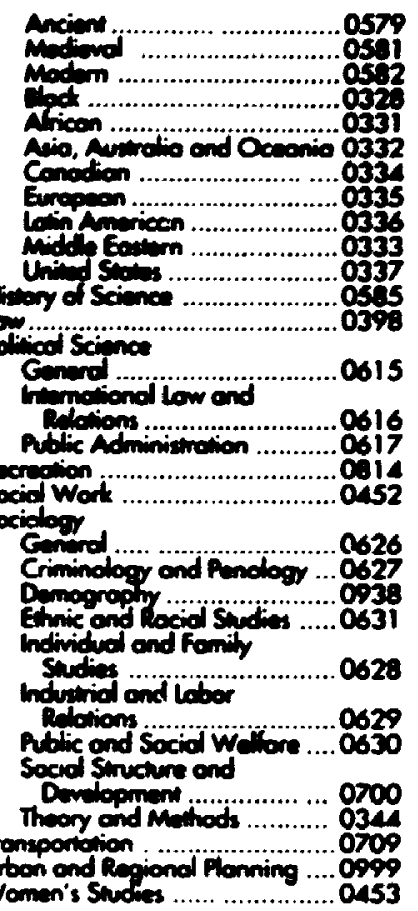

$\begin{array}{llll}\text { Spench Pathology } & \ldots . & \ldots & .0460 \\ \text { Toxicology } & \ldots & \ldots & .0383\end{array}$ Home Economues ................. 0386

\section{mrexcu scances}

Pure scinences

Chemisty

Gemerd.. 0485

Acricumured. .......... .. 0749

Andyeol ............... . . . . .

Diochiminghy ................ 0487

Inorronic . ................... 0489

Oreonic.. ...... ............ 0490

Finecuried .................0491

Finzicel ......................... 049

Pofmer ........................045

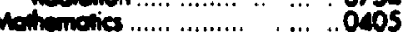

Pintives

Generd.. ........... 0405

Acountics ..... ....... 0 .786

Atironery end

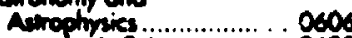

Amespherie Science. . ......... O40

Alomic ......................... O74

Etchonic ond El cotricity .....0607

Enmion Porictes and 079

fuidend fry .................. .07\%

Malader ...........................6\%

Nuctor ............................ OS10

Opics ............................ 075

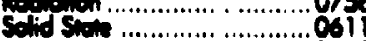

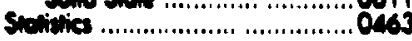

Antind Saines:

Apolind Muchonict

Cempiner Science

.034
Enginatring

Generol ....... .............0537

Ampopoce ................ 0538

Agriecthurol .......................

Auromotive ..................... 0540

Biomendicol ................ OSA

Chemicel ....................0542

Civil .........................

Electronics ond Einctricol....... 054

Aptratic . . ...................... .

Industrial .........................05

Morime .................. OSA7

Molertols Science ...............0794

mochonicel.. .............0548

Mathory ... . ................ 0743

Mining ...........................055

Nuctor ......................055?

Podedoging . ......................0Sd9

Pamolom

Sonitury ond Municipol ...... Oss

Goouchnology ...................... O42

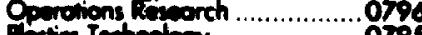

Plouties Technology ................. Orgs

crovicar

Gomed ……..................821

Chinied

Demopomenoil ........................... 0620

Experimentel .............................0623

Intwied .................................628

perendity i...........................825

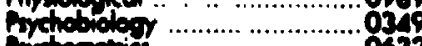

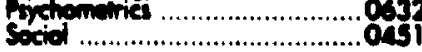


The undersigned hereby recommend to the Faculty of

Graduate Studies and Research acceptance of the thesis

\title{
Measurement and Performance Analysis of World Wide Web Applications
}

\author{
submitted by Jie Ma
}

in partial fultillment of the requirements

for the degree of Master of Engineering

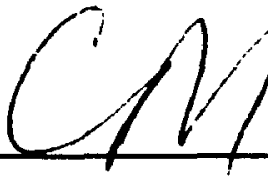

Professor C. M. Woodside, Thesis Supervisor

\section{Chair, Department of Systems and Computer Engineering}

Department of Systems and Computer Engineering

Faculty of Engineering

Carleton University

January 19, 1996 


\section{Abstract}

Among the internet applications, World Wide Web (WWW) applications have become the number 1 of traffic of internet backbone. It is necessary to study the impact of WWW application on the system performance. Measurement and performance modelling techniques were employed for both the server and client side in this thesis. Mosaic was chosen as the client target since it was the most popular and most powerful WWW browser when this project was started. A experimental version of Mosaic with a software monitor was created for measurement. With the measured data, a User Behavior Graph model of Mosaic was built based on the users actions which are described as states. By solving the model, the transition probabilities among the states, and the system resource demands for each state are obtained. This model provides information about the user's behavior and the workload parameters of the program which are used for an analytic performance model of the WWW system. The performance model also used data from the WWW Server in the Department of System and Computer Engineering. This analytic performance model described here appears to be the first performance model of an entire WWW system up to the time of writing the thesis. It gives the performance ana!ysis of the WWW system with both remote and local user requests. Performance was studied with different number of users, different rates of remote requests. different size of file transfer and different caching hit ratio. 


\section{Acknowledgments}

I would like to thank Professor C.M. Woodside for his invaluable support and guidance during the time in Carleton University. His support is not only in the guidance of the research work, but also the encouragement of completing this thesis.

Very special thanks to my daughter, Yao Ma, for her more care of herself. The time she saved for me is much valuable during my study.

I also wish to thank Dr. Greg Franks, Dr. Vidar Vetlard, Mr. Narendra Mehta. Professor Jerome Rolia and Mr. Alex Hubbard for their help. 


\section{Table of Contents}

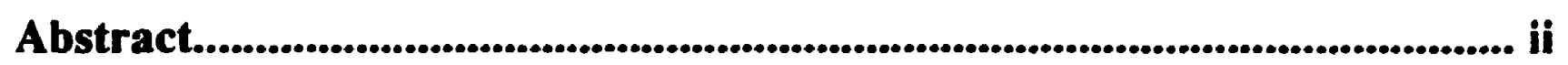

Acknowledgments . iii

Table of Contents iv

List of Figures vii

List of Tables xi

Chapter 1 Introduction 1

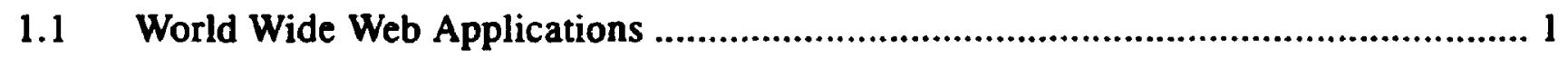

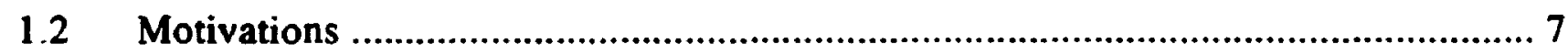

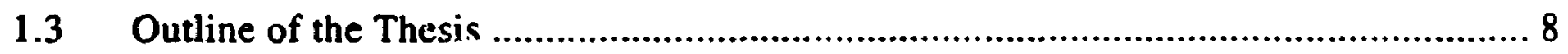

Chapter 2 Background Information ............................................................ 10

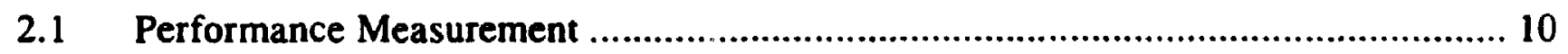

2.2 Workload Characterization and User Behavior Gragh ...................................... 15

2.3 System Performance Modelling ……..................................................................... 19

2.4 Measurement And Performance Research with World Wide Web applications ........ 21

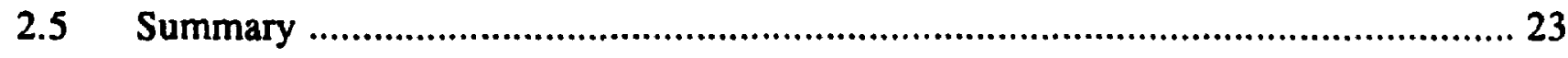

Chapter 3 Measurement of Mosaic ................................................................... 24

$3.1 \quad$ The Design and Techniques of Measurement .......................................................... 24 
3.2 Implementation of the experimental version of Mosaic ................................. 26

3.3 Description of Logging Data in Mosaic .......................................................... 30

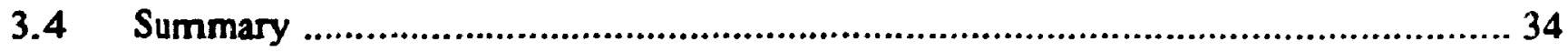

Chapter 4 User Behavior of Mosaic ....................................................\$5

4.1 The Definition of User Behavior Graph of Mosaic: Mosaic Model ........................ 35

4.2 Transition Probabilities of Mosaic Model ............................................................. 39

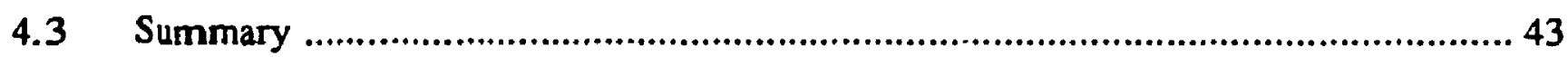

Chapter 5 Parameters and Some Metrics of Mosaic Model .................... 44

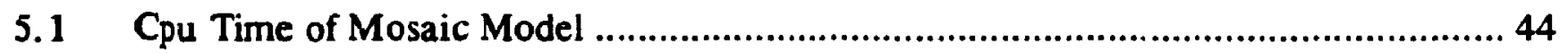

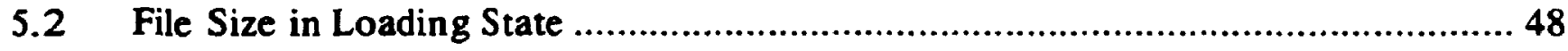

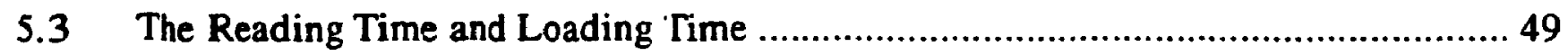

5.4 Other Parameters of Mosaic Model …............................................................... 51

5.5 Response Cpu time, Response Rate and Utilization of Mosaic ............................53

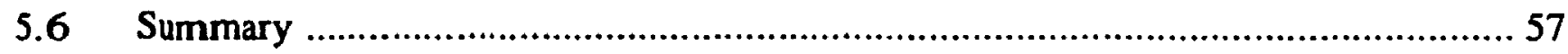

Chapter 6 The Performance Model of WWW System .............................. 58

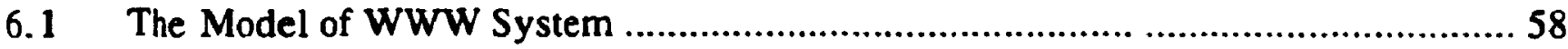

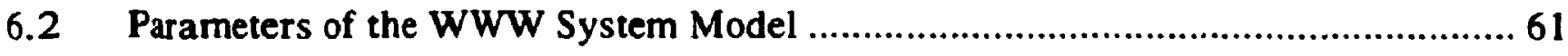

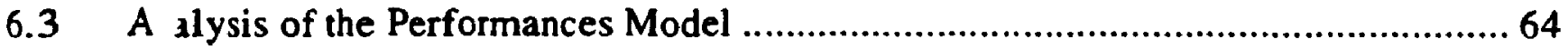

6.4 Some Issues About the Parameters and Performance ......................................... 78

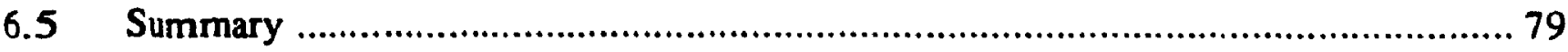

Chapter 7 The Influence of System Parameters on Performance of WWW System Model ................................................................ 81 
7.1 The Performance With Different File Sizes ........................................................81 81

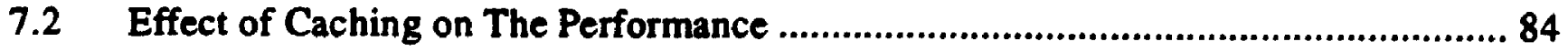

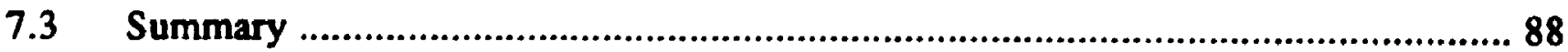

Chapter 8 Conclusions and Future Studies ............................................... 89

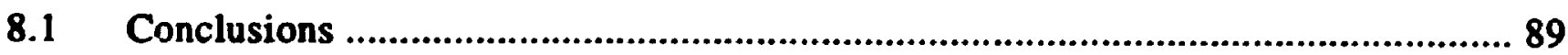

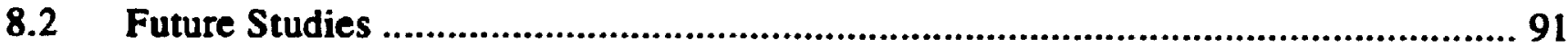

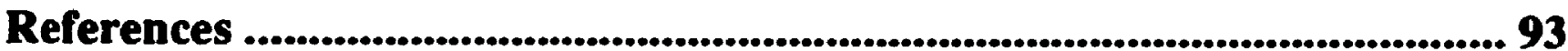

Appendix A. Common State Occurrences and Their Probabilities of Occurrences in Mosaic Model ........................................ 98

Appendix B. Cpu Time Distributions of Mosaic Model ........................ 99

Appendix C. State Parameters of Mosaic Model ............................... 103

Appendix D. More Performance Data of WWW System Model ....... 112 


\section{List of Figures}

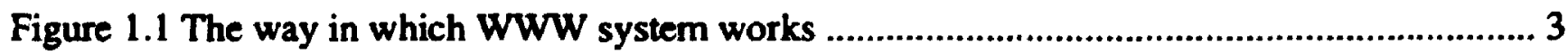

Figure 1.2 Architecture of WWW system ...................................................................................... 4

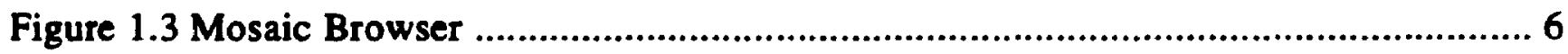

Figure 2.1 Layered view of a distributed-system monitor ............................................... 13

Figure 2.2 Steps in program execution monitoring ............................................................ 14

Figure 2.3 Procedure of workload characterization ............................................................. 16

Figure 2.4. Example of User Behavior Graph with three nodes ...................................... 18

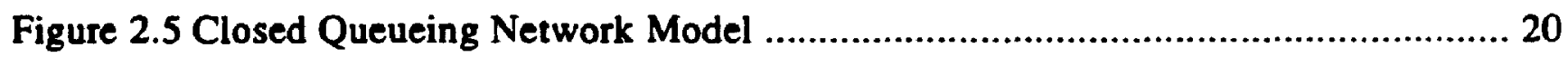

Figure 3.1 Measurement design of WWW applications ............................................................... 25

Figure 4.1 User Behavior Graph of Mosaic ................................................................... 36

Figure 4.2 User Behavior Graph of Mosaic with transition probabilities ......................... 42

Figure 5.1. Cpu Time Distribution of State 3 (main window) ........................................... 46

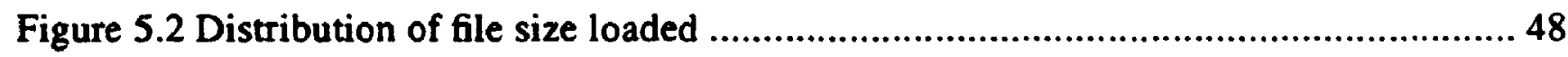

Figure 5.3. The Reading Time In State 3 (Main Window). Mean:37.0125 ......................... 50

Figure 5.4. The Loading Time In State 2 (Loading) .......................................................... 50

Figure 6.1 Performance Model of WWW System ........................................................... 59

Figure 6.2.1. Response time of remote request $\mathrm{Xr}$ vs remote arrival rate $\mathrm{Fr}$,

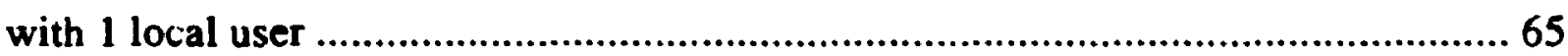

Figure 6.2.2. Total request throughput $\mathrm{Xs}$ vs remote arrival rate $\mathrm{Fr}$.

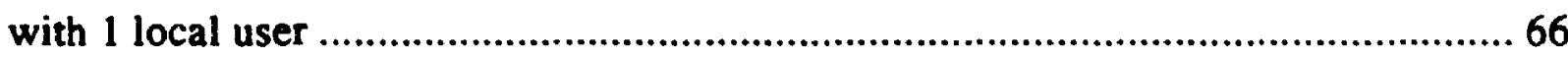

Figure 6.2.3. Local request throughput $\mathrm{Xl}$ vs remote arrival rate $\mathrm{Fr}$,

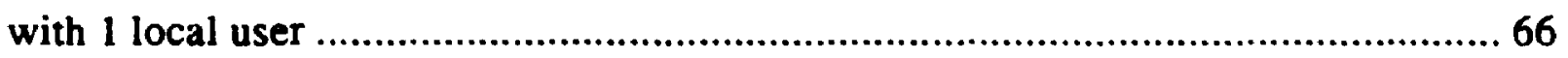

Figure 6.2.4. Utilizations of the server $U s$ and disk $U d$. vs remote arrival rate $F r$,

with 1 local user

Figure 6.3.1. Response time of remote request $R r$ vs remote arrival rate $F r$,

with 100 local users

Figure 6.4.1. The difference of remote response time of 1 local user

from 100 local users, vs the remote arrival rate Fr ................................................... 70 
Figure 6.5.1. Response time of remote requests $\mathrm{Rr}$ vs the number of local users $\mathbf{N}$ with remote arrival rate 0.001

Figure 6.5.2. Total request throughput $\mathrm{Xs}$ vs the number of local users N1, with remote arrival rate 0.001

Figure 6.5.3. Local request throughput $\mathrm{Xl}$ vs the number of local users $\mathrm{Nl}$, with remote arrival rate $\mathbf{0 . 0 0 1}$

Figure 6.5.4. Utilizations of the server Us and disk Ud, vs the number of local users N1 with remote arrival rate 0.001

Figure 6.6.1. Response time of remote requests $\mathrm{Rr}$ vs the number of local users $\mathrm{Nl}$, with remote arrival rate 3

Figure 6.7.1. The difference of remote response time of remote arrival rata 0.001 and 3

vs the number of local users

Figure 7.1.1. Response time of remote requests $\operatorname{Rr}$ vs the number of packets

of a request PK, with 28 local users and remote arrival rate 0.5

Figure 7.1.2. Response time of remote requests $\operatorname{Rr} v$ s the number of packets

of a request $P K$, with 100 local users and remote arrival rate 1.7

Figure 7.2.1. Response time of remote request $\mathrm{Rr}$ vs remote arrival rate $\mathrm{Fr}$, with 100 local users and caching hit ratio $C=0,0.1,0.2,0.3$

Figure B.1 Distribution of cpu time of "Start" state,

5 equal cells in the range $(1.34,3.76)$ seconds

Figure B.4. Distribution of cpu time of "Submit Form",

? equal cells over $(0,0.01)$ seconds

Figure B.2. Distribution of cpu time of "Loading" state,

23 equal cells in the range $(0,2.3231)$ seconds

Figure B.3. Distribution of cpu time of "Main Window",

29 equal cells in the ranger $(0,0.5139)$ seconds

Figure B.5. Distribution of cpu time of "Open URL" state,

9 equal cells over, $(0.01,0.4878)$ seconds

Figure B.6. Distribution of cpu time of "Reload" state,

14 equal cells over $(0,0.019)$ seconds

Figure B.7. Distribution of cpu time of "Home", 
3 equal cells over $(0,0.02)$ seconds

Figure B.8 Distribution of cpu time of "Active Anchor" state,

9 equal cells over $(0,0.7884)$ seconds

Figure B.9. Distribution of cpu time of "Back" state,

10 equal cells over $(0.0161,0.8177)$ seconds

Figure B.10. Distribution of cpu time of "Forward" state,

8 equal cells over $(0.0162,0.5011)$ seconds.

Figure B.11. Distribution of cpu time of "Hotlist",

17 equal cells over $(0,0.1666)$ seconds 102

Figure B.12. Distribution of cpu time of "Hotlist",

6 equal cells over $(0.04,0.562)$ seconds

Figure B.13. Distribution of cpu time of "Add Hotlist",

5 equal cells over $(0.01,0.05)$ seconds

Figure B.14. Distribution of cpu time of "Printing" state,

18 equal cells over $(0.09,1.2647)$ seconds

Figure B.15. Distribution of cpu time of "Saving" state.

10 equal cells over $(0.08,0.9397)$ seconds

Figure B.16. Distribution of cpu time of "Exit" state.

12 equal cells over $(0,1.3538)$ seconds

Figure D.1.1. Response Time of remote requests $\mathrm{Rr}$ vs arrival rate Fr.

with 500 local user

Figure D.1.2. Total request throughput Xs vs arrival rate Fr.

with 500 local user

Figure D.1.3. Local request throughput $\mathrm{Xl}$ vs arrival rate $\mathrm{Fr}$,

with 500 local user

Figure D.1.4. Utilizations of the server Us and disk Ud, vs remote arrival rate Fr. with 500 local user

Figure D.2.1. Response time of remote requests $\mathrm{Rr}$ vs the number of local users $\mathrm{Nl}$, with remote arrival rate 5

Figure D.2.2. Total request throughput $\mathrm{Xs}$ vs the number of local users NI, with remote arrival rate 5 
Figure D.2.3. Local request throughput $\mathrm{XI}$ vs the number of local users $\mathrm{NI}$ with remote arrival rate 5

Figure D.2.4. Utilizations of the server Us and disk Ud, vs the number of local users $\mathrm{Nl}$ with remote arrival rate 5 116

Figure D.3. Response time of remote requests $R r$ vs the number of packets of a request, PK, with 280 local users and remote arrival rate 5 116

Figure D.4.1. Response time of remote request $R r$ vs remote arrival rate $F r$, with 100 local users

Figure D.4.2. Total request throughput $\mathrm{Xs}$ vs remote arrival rate $\mathrm{Fr}$, with 100 local users

Figure D.4.3. Local request throughput $\mathrm{Xl}$ vs remote arrival rate $\mathrm{Fr}$, with 100 local users

Figure D.4.4. Utilizations of the server Us and disk Ud, vs remote arrival rate $\mathrm{Fr}$ with 100 local users

Figure D.5.1. Response time of remote requests Rr vs the number of local users Nl, with remote arrival rate 3

Figure D.5.2. Total request throughput Xs vs the number of lucal users N1. with remote arrival rate 3

Figure D.5.3. Local request throughput $\mathrm{Xl}$ vs the number of local users NI. with remote arrival rate 3

Figure D.5.4. Utilizations of the server Us and disk Ud, vs the number of local users $\mathrm{Nl}$ with remote arrival rate 3

Figure D.6.1. Response time of remote request $R r$ vs remote arrival rate Fr, with 100 local users and caching hit ratio $\mathrm{C}=0.0 .1,0.2,0.3$

Figure D.6.2. Total request throughput $\mathrm{Xs}$ vs remote arrival rate $\mathrm{Fr}$, with 100 local users and caching hit ratio $\mathrm{C}=0,0.1,0.2,0.3$

Figure D.6.3. Local request throughput $\mathrm{Xl}$ vs remote arrival rate $\mathrm{Fr}$, with 100 local users and caching hit ratio $\mathrm{C}=0,0.1,0.2,0.3$

Figure D.6.4. Utilizations of the server Us and disk Ud, vs remote arrival rate Fr, with 100 local users and caching hit ratio $C=0,0.1,0.2,0.3$ 


\section{List of Tables}

Table 1.1. Statistics of WWW Traffic in NSFNET Backbones ............................................... 2

Table 4.1 Meaning of the states in Mosaic model (Figure 4.1) ......................................... 37

Table 4.2. Transition Occurrences From State $i$ to state $j$...................................................... 40

Table 4.3 Transition Probabilities From State i to State j ....................................................... 41

Table 5.1. Cpu Times of All States in Mosaic Model(seconds) ........................................... 45

Table 5.2 Rank of states according to the cpu time from high to low ............................. 46

Table 5.3. Parameters of State 3 (Main Window) in Mosaic Model ...................................... 53

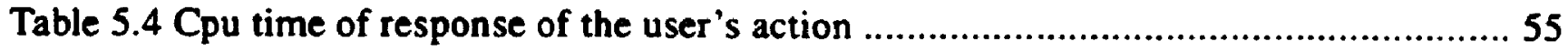

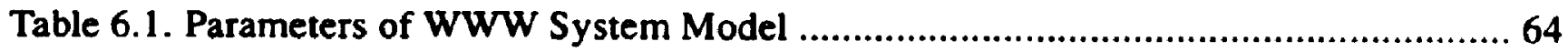

Table 7.1. Disk demands and saturation of remote arrival rate with caching ...................... 85

Table 7.2 The performance metrics at the pointer $\operatorname{Rr}=2$ seconds, local users is 100 ,

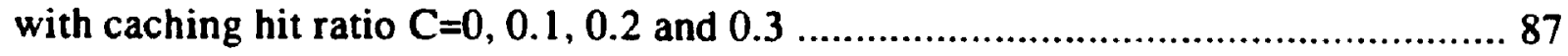

Table 7.3 Improvement of the WWW system with caching,

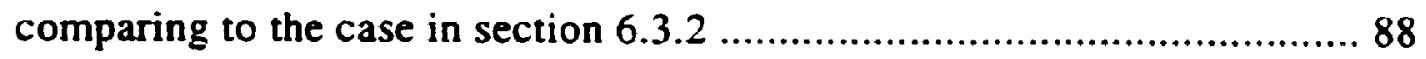

Table A.1. State Occurrences Probabilities of in Mosaic Model ............................................ 98

Table C.1 Parameters of State 1 (Start) in Mosaic Model .................................................. 104

Table C.2 Parameters of State 2 (Loading) in Mosaic Model ............................................. 104

Table C.3 Parameters of State 3 (Main Window) in Mosaic Model ..................................... 105

Table C.4 Parameters of State 4 (Submit Form) in Mosaic Model ...................................... 105

Table C.5 Parameters of State 5 (Open Url) in Mosaic Model ............................................. 106

Table C.6 Parameters of State 6 (Reload) in Mosaic Model ............................................ 106

Table C.7 Parameters of State 7 (Home) in Mosaic Model ................................................... 107

Table C.8 Parameters of State 8 (Active Anchor) in Mosaic Model .................................... 107

Table C.9 Parameters of State 9 (Back) in Mosaic Model .................................................. 108

Table C.10 Parameters of State 10 (Forward) in Mosaic Model ......................................... 108

Table C.11 Parameters of State 11 (Key Press) in Mosaic Model ......................................... 109

Table C.12 Parameters of State 12 (Hotlist) in Mosaic Model ................................................ 109

Table C.13 Parameters of State 13 (Add Hotlist) in Mosaic Model ..................................... 110 
Table C.14 Parameters of State 14 (Printing) in Mosaic Model 110

Table C.15 Parameters of State 15 (Saving) in Mosaic Model 111

Table C.16 Parameters of State 16 (Exit) in Mosaic Model 


\section{Chapter 1}

\section{Introduction}

Performance is a key criterion in the design, procurement, and use of computer systems. Computer system users, administrators, and designers are all interested in getting the highest performance of their systems for a given cost. As such, system performance analysis is an important research area in computer systems.

The World Wide Web (WWW) [1] [2] [3] is one of the most important and most popular information exchange system in the internet, and it is growing dramatically. The impact of $W W W$ application to the system performance is significant. Therefore, the research on the performance analysis is necessary for the design and use of computer system.

This thesis is my research of the measurement and performance analysis of WWW applications. It includes the workload characterization of the client side and the analytic performance model of the WWW system including both of ciient and server. The user behavior is also studied due to its importance for further development of WWW system.

\subsection{World Wide Web Applications}

As mentioned, we do the research with $W W W$ because $W W W$ is one of the most powerful and most popular information exchange applicat.uns in the internet. It is growing the fastest among the popular internet service. Table 1.1 gives some statistical data of traffic in the NSFNET (National Science Foundation Network) backbones, which are reported by Merit Network Information Center Services [4]. It shows both the traffic counts and the rank (among the service like FTP, TELNET, NNTP, GOPHER and so on). 


\begin{tabular}{|c|c|c|c|c|c|c|}
\hline Month & Rank & Packet Count & \& Pkts & Rank2 & 2 Byte Count & 8 Byts \\
\hline$=========$ & $====$ & 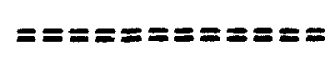 & : = ==:= = & $====$ & 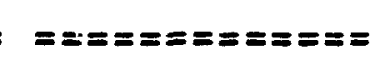 & $======$ \\
\hline Jan. 1994 & 11 & 822317950 & 1.487 & 8 & 269129084100 & 2.614 \\
\hline Feb. 1994 & 11 & 1105399700 & 1.843 & 7 & 347503518500 & 3.044 \\
\hline Mar. 1994 & 10 & 1597847800 & 2.297 & 6 & 518083617700 & 3.694 \\
\hline Apr. 1994 & 8 & 2035281650 & 2.846 & 6 & 671950150550 & 4.695 \\
\hline May. 1994 & 8 & 2589730950 & 3.403 & 5 & 799162732200 & 5.170 \\
\hline Jun. 1994 & 7 & 3060869850 & 4.066 & 5 & 946538669550 & 6.166 \\
\hline Jul. 1994 & 7 & 3355432550 & 4.477 & 41 & 1056081229600 & 6.622 \\
\hline Aug. 1994 & 6 & 4187132200 & 5.248 & 31 & 1311822496550 & 7.930 \\
\hline Sep. 1994 & 5 & 5082138200 & 5.701 & 31 & 1593463753750 & 8.557 \\
\hline Oct. 1994 & 5 & 7009616350 & 7.000 & 32 & 2152956666650 & 10.157 \\
\hline Nov. 1994 & 3 & 10402528150 & 9.755 & 23 & 3126195255050 & 13.917 \\
\hline Dec. 1994 & 2 & 11672375900 & 11.708 & 23 & 3475374706500 & 15.978 \\
\hline Jan. 1995 & 2 & 11518306800 & 13.122 & 23 & 3382697720400 & 17.693 \\
\hline Feb. 1995 & 2 & 11199998500 & 15.092 & 23 & 3266211378200 & 19.533 \\
\hline Mar. 1995 & 1 & 16880373100 & 19.075 & 24 & 4833271270950 & 23.880 \\
\hline Apr. 1995 & 1 & 12694282100 & 21.443 & 13 & 3518742182400 & 26.250 \\
\hline
\end{tabular}

Note:

(i) The traffic counts started to decrease from December 1994 because the networks began to migrate to the new NSF network architecturs. The last report is April, 1995.

(ii) Data source: gopher://nic.merit.edu:7043/1/nsfnet/statistics

(iii) "Rank" is the rank of different services in term of packets counted

(iv) "Ranks" is the rank of different services in term of bytes counted.

Table 1.1. Statistics of WWW Traffic in NSFNET Backbones

From Table 1.1, we can sce that WWW traffic became the number 1 internet service in March, 1995. It occupies $21.443 \%$ of packet transmitted and $26.250 \%$ of total traffic respec- 
tively.

WWW is the universe of network-accessible information, an embodiment of human knowledge. It is an initiative started at CERN, and now there are many participants. It has a body of software and a set of protocols (HTTP, FTP, NNTP, GOPHER, WAIS, TELNET, RLOGIN, and etc.).

WWW uses hypertext and multimedia techniques to make the web easy for anyone to roam, browse, and contribute to. The way in which WWW system works is shown in Figure 1.

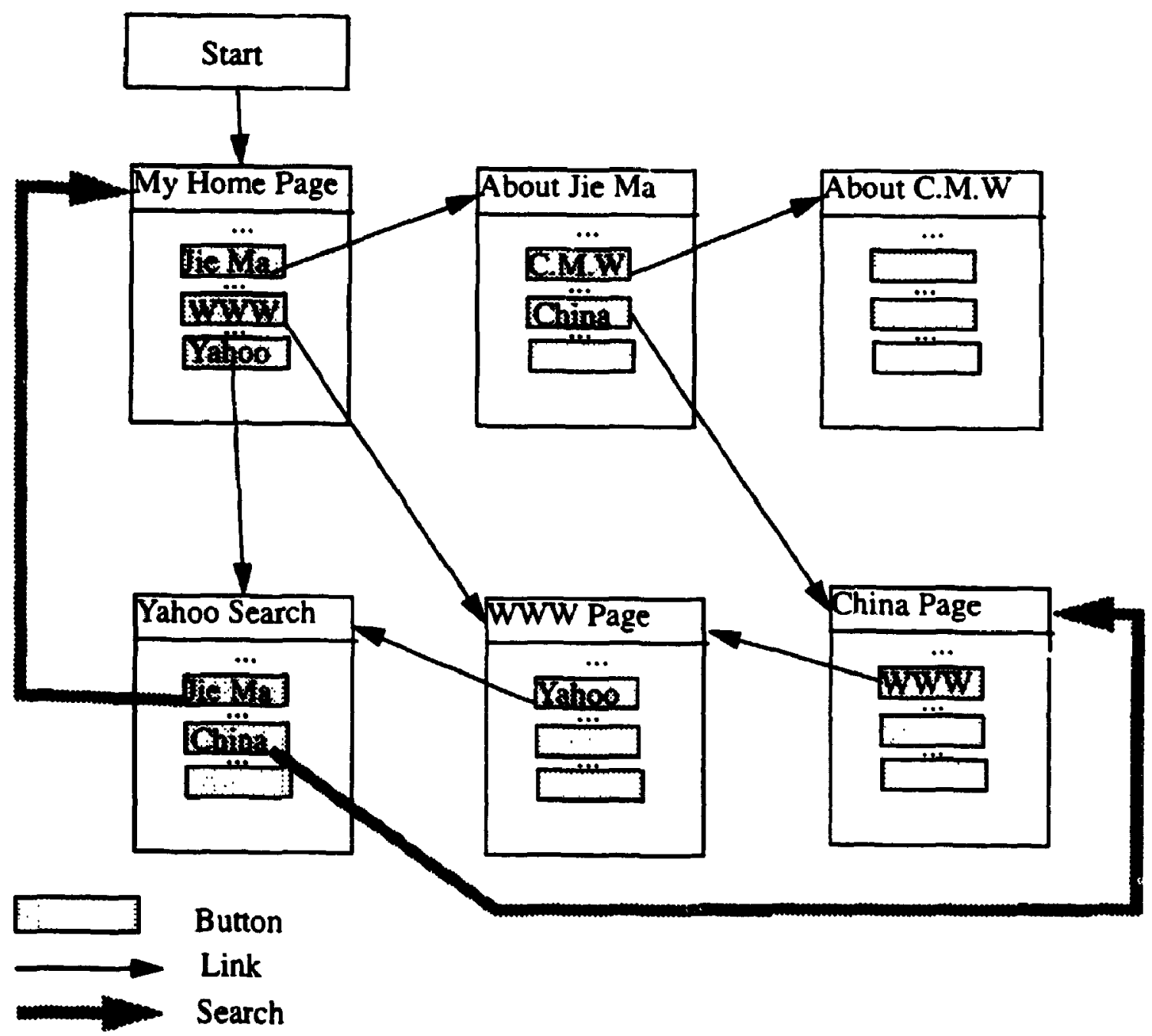

Figure 1.1 The way in which WWW system works 
The files the WWW system accesses are hypertext documents in HTML [5] [6] [7]. The first document loaded at the beginning is the Home Page. The documents refer to each other by links which appear in the document as buttons. Through links, the user can access other documents by pushing the buttons in the present document. From its similarity to a spider's construction, this system is called the Web which links the world's hypertext documents.

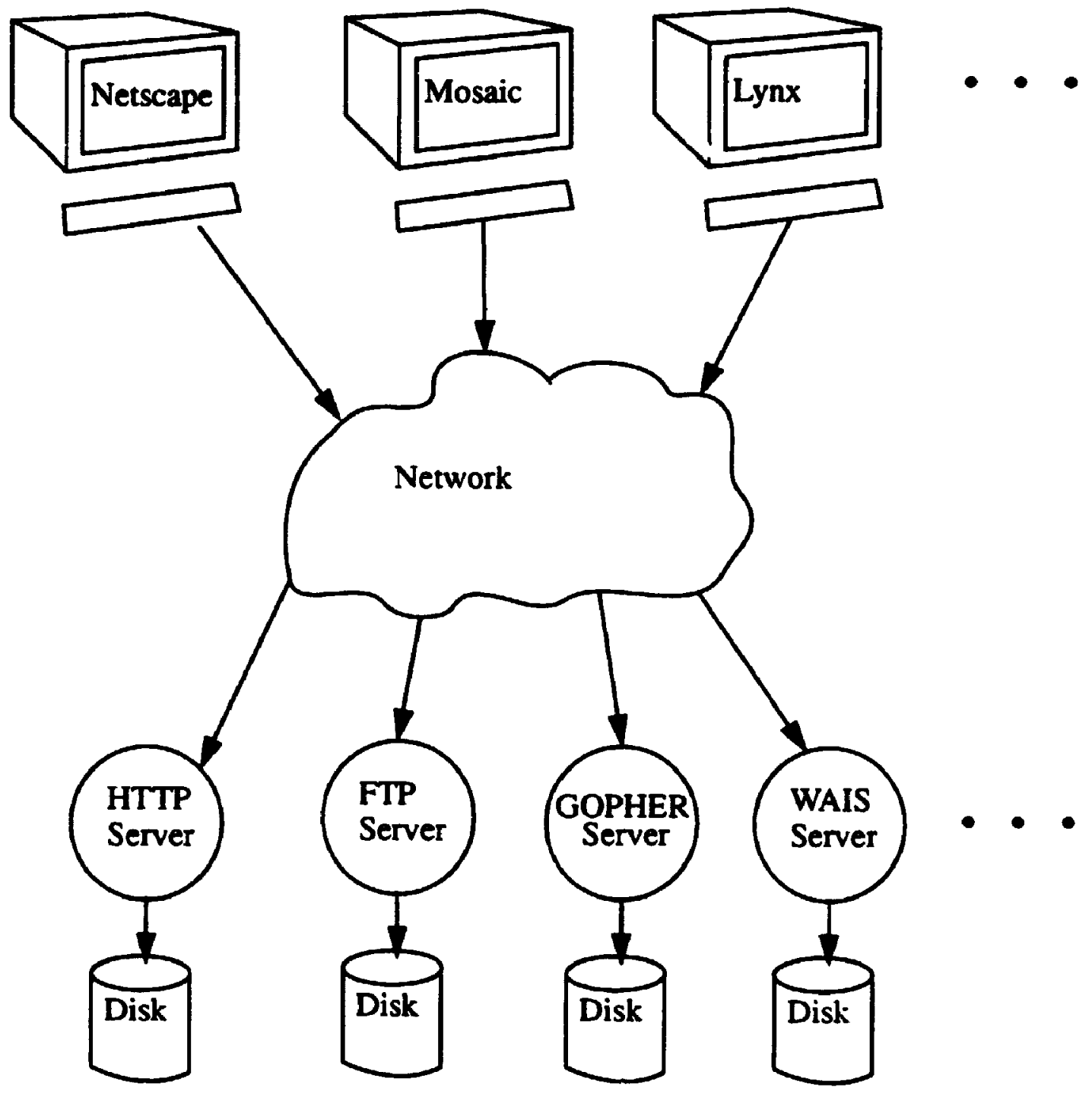

Figure 1.2 Architecture of WWW system

WWW works in client/server model. The architecture of WWW (Figure 1.2) uses browsers 
(clients) which know how to access data from the servers and present the data to users from the HTML documents, and servers which know how to extract data and provide them to clients. The WWW servers are HTTP [8] servers which are installed at many sites. A HTTP server is a daemon which provides hypertext and multimedia information to clients. Usually, a HTTP server manipulates a number of documents through its own disk. The users don't need to know from which server the document is loaded. In addition to HTTP servers, WWW also can access any data in non-WWW servers (FTP, GOPHER, WAIS or NNTP) as WWW clients have the ability to present all such data as hypertext.

The information we can get from WWW includes anything served through ftp server, gopher server, usenet, telnet, hytelnet, hyper-g, library system, CSO, X.500, whois. finger, techinfo, textinfo, man page formed info, html-formatted hypertext and hyperniedia documents. Various search engines are included through the above services. For example, the "Yahoo Search" is a very powerful $W W W$ search engine. The information which can be retrieved includes business inform, academic institution, government and public service information, individual home pages, technology resources in different area, news. weather, public discussion. and so no.

There are different WWW browsers. When the research began. Mosaic [9] was one of the most popular browsers. It is a graphic window oriented system like $\mathrm{X}$ windows, as shown in Figure 1.3. A user uses a mouse to select anchors of the window to retrieve other hypertext/ multimedia documents either in the same server or different server. The users can also select command buttons in the Mosaic window to invoke different functions, e.g. save document, maintain hotlist, open a user specified document and so on.

Lynx [10] is another WWW browser which is based on text windows like vt100 terminal. Many users who'don't have window system get benefit from Lynx. Instead of using a mouse, the user can use keyboard to choose different link buttons in the hypertext document, or to choose different command in the lynx window. In our study, measurement for lynx was also done in the same way as for Mosaic. However, we only chose Mosaic as a case in this thesis since Mosaic is more popular than Lynx. 


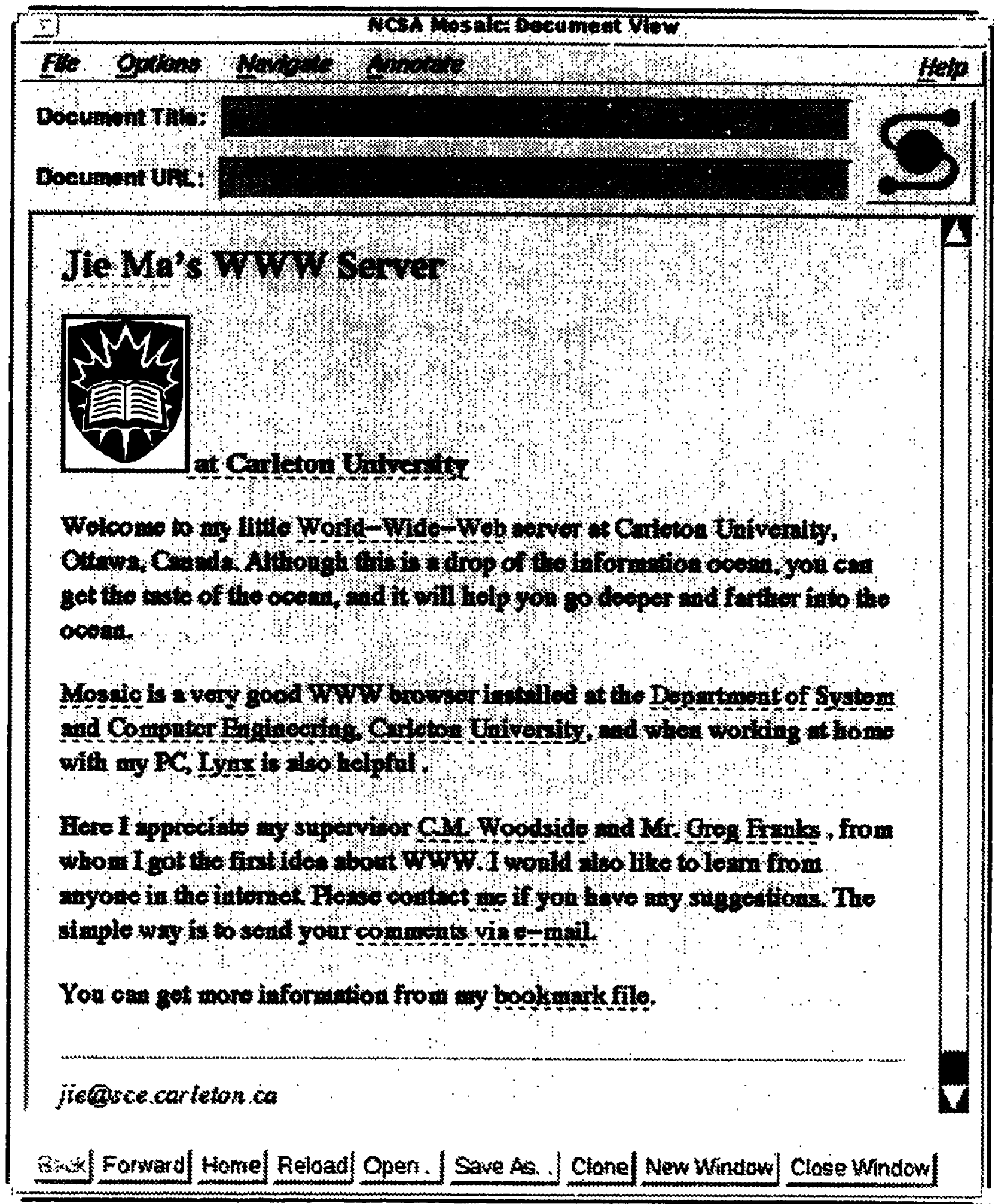

Figure 1.3. Mosalic Browner 


\subsection{Motivations}

Because of the growth of use, $W W W$ has become an important workload component. It will be helpful to understand the workload generated by user of WWW applications. This thesis describes the measurement and performance analysis of $W W W$ applications.

Among the three analysis techniques, measurement, simulation, and analytic modeling, measurement is the most costly and most difficult among the analysis techniques since it requires real system, real software, real users and more time. However, if measurement is under the real system's conditions, we can get the highest accuracy and saleability. Furthermore, measurement results can be used for the validation and further studies of analytic or simulation models. The accuracy of the parameters measured is very important for the analytic modelling and simulation solution. We will build a performance model of WWW system. Most of the parameters in the model are either from our measurement or from other statistical data.

The objective of this research is to study the general question of performance on WWW systems and identify important issues. The study has 3 aspects.

The first aspect of the study is to model the behaviors of users of Mosaic browser. This is necessary when WWW applications are workload components in any of the three analysis techniques mentioned above. Most of the operations and executions of WWW are selected depending on the user behavior since WWW clients are user interactive applications.

The second aspect of the study is to describe the measurement metrics and system parameters, including service time and some other system parameters in the Mosaic model. These parameters are represented in the system resource demands based on the user's actions. They can be used in analytic or simulation model for future study. As such, the first and the second aspects are about the workload characterization, which allow us io build a performance model. 
The third aspect of the study is a queuing network model of WWW application, including Mosaic client and WWW server. Performance with different local users and different remote request rate will be studied. This model could be used by a system analyst or planner to analyze the performance of a particular system which run a WWW server. This mainly study the impact of system performance with WWW server. Most of the system parameters of the model are from the Mosaic model we built and from measurement of the server. Other parameters are from the internet statistical data. It appears that this is the first performance model of WWW application suitable for prediction.

\subsection{Outline of the Thesis}

This thesis consists of eight chapters, references and appendices.

Chapter 2 gives the brief view of the study area related to this thesis project, which are mainly from the papers that have been published.

Chapter 3 describes the implementation of the experimental version of Mosaic as a tool for the purpose of the thesis, which is modified from the original Mosaic. The events measured and the logging data are explained in this chapter.

Chapter 4 is one of the main topics of the thesis. An User Behavior Graph model will be defined. The dynamic and static parameters of the model will be measured in this chapter. The state transition probabilities will be obtained.

Chapter 5 analyzes the system resource demands and some measurement metrics. These pa-ameters also are the workload parameters of Mosaic. This will make a useful workload model.

Chapter 6 is an analytical performance model of WWW system. Qnap2 is employed to solve the model. Performance will be studied with different users and different remote arrival rate of requests. 
Chapter 7 is the further study of the performance model of WWW system. The impacts of different file sizes and of different caching hit ratio on the system performance will be studied.

Chapter 8 gives the conclusion of the thesis and discusses the further studies of this project.

Finally, the references and appendices are given. Appendix A, B and $C$ are the additional measured and statistical data of Mosaic, which are related to Chapter 4 and Chapter 5. Appendix $D$ is the additional performance data of WWW model from Chapter 6 and 7.

The author's contributions in the thesis include:

1. Instrumentation of Mosaic and Lynx programs for logging data

2. Measurement of parameters of both of client and server

3. Statistical reduction of the logging data

4. Mosaic user model, with the users' behavior

5. Mosaic program model with the service time and other system parameters

6. Analytic performance model of WWW system with both client and server 


\section{Chapter 2}

\section{Background Information}

It is necessary to have a brief review of the background related to this thesis. In this chapter, we will describe the measurement and analytic modelling of system performance. The issues related to the workload characterization are described. This will focus on the User Behavior Gragh since it is suitable to client workload characterization of WWW system. And it also give more detailed information about the users behavior for software development of $W W W$ system. Finally, the research work of the measurement and modelling of WWW system is surveyed. This will give us a outline of the research of system performance with WWW system. The chapter simply gives a very brief review in our research area. Most of issues and concepts are summarizes from papers and publications. Others are from internet resources through the WWW browser.

\subsection{Performance Measurement}

As we know, measurement is the most costly and most difficult one among the three major performance evaluation techniques (measurement, simulation and analytic modelling). However, the result of the performance evaluation from the measurement would be more accurate and more valuable, with proper measurement methodologies and tools [11] [12] [13]. Measurement techniques are used with existing systems. This relies on knowledge of the hardware, software, workload, and monitoring tools associated with the system under study. It also requires access to information recorded by accounting and software monitors during system operation.

A Monitor is a tool used to measure the activities and events on a system. It observes the performance of systems, collects performance statistics, analyzes the data, and display results. Monitoring is the first and the key step in performance measurements. 
There are different monitors, such as software monitors, hardware monitors, firmware monitors, or hybrid monitors which is a combination of hardware, software or firmware. A monitor may be event driven or timer driven (sampling monitor). A brief overview of different types of monitors will be described here.

\subsubsection{Software Monitors}

Software monitors are used to monitor operation systems and higher level software such as networks and databases. The method is instrumentation. Some special instrumentation instructions are inserted in the source code of the application, and are executed with certain event, processor trace or timer interrupt. The data obtained are either displayed on-line or are saved for later analysis.

Software monitors generally have lower input rates, lower resolutions, and higher overhead than hardware monitors. However, they have higher input widths and higher recording capacities than hardware monitors. They are easier to develop and easier to modify. if necessary.

\subsubsection{Hardware Monitors}

A hardware monitor consists of separate pieces of equipment that are attached to the system being monitored via probes. No system resources are consumed in monitoring. Thus. hardware monitors generally have lower overhead than software monitors. Their input rate is also higher. Further, the probability of their introducing bugs into the system operation is generally lower than that of software monitors. However, they are expensive to buy, difficult to connect, and they cannot associate physical events with the application or context that caused them.

\subsubsection{Firmware Monitors}

Firmware monitors are implemented by modifying the processor microcode. These are useful 
for applications that fall between the software and hardware monitoring boundaries. However, since the microcode space is limited and there are tighter timing limitations, firmware monitors generally do very limited data reduction.

\subsubsection{Hybrid Monitors}

A monitor using a combination of software, hardware, or firmware is hybrid monitor. Software monitors can associate events with application-level operations and have good data reduction capabilities, while hardware monitors have high resolution. Thus, a hybrid monitor, consisting of a hardware data-gathering component together with a software data reduction component, provides the best of both worlds.

\subsubsection{Distributed-System Monitors}

Monitoring a distributed system is more difficult than monitoring a centralized system. The monitor itself must be distributed and shoul $\AA$ consist of several components that work separately and concurrently. These $\mathrm{c}^{\sim}$ mponents of a distributed-system monitor can be divided into a number of layers according to their functions, as shown in Figure 2.1.

The Observation layer gathers raw data on individual components of the system Normally, each component may have an observer designed specifically for it. Thus, there may be several observers located on different subsystems. Collection layer collects data from various observers. It is possible to have more than one collector on large systems. Analysis layer analyzes the data gathered at various collectors. It may consist of various statistical routines to summarize the data characteristics. Presentation layer deals with human user interface. It produces, for example, reports, Jisplays, and alarms. Interpretation layer refers to the intelligent entity (usually a human being or an expert system) that can make meaningful interpreta tions of the data. Console layer provides an interface to control the system parameters and states. Management layer makes the decision to set or change system parameters or configurations based on interpretation of monitored performance. Managers are assigned to this layer. A distributed-system monitor may consist of multiple (zero or more) components from 
each of the layers. Thus, there is a many-to-many relationship between successive layers. Most of distributed-system monitors are hybrid and make use of software, hardware, and firmware as well as human beings.

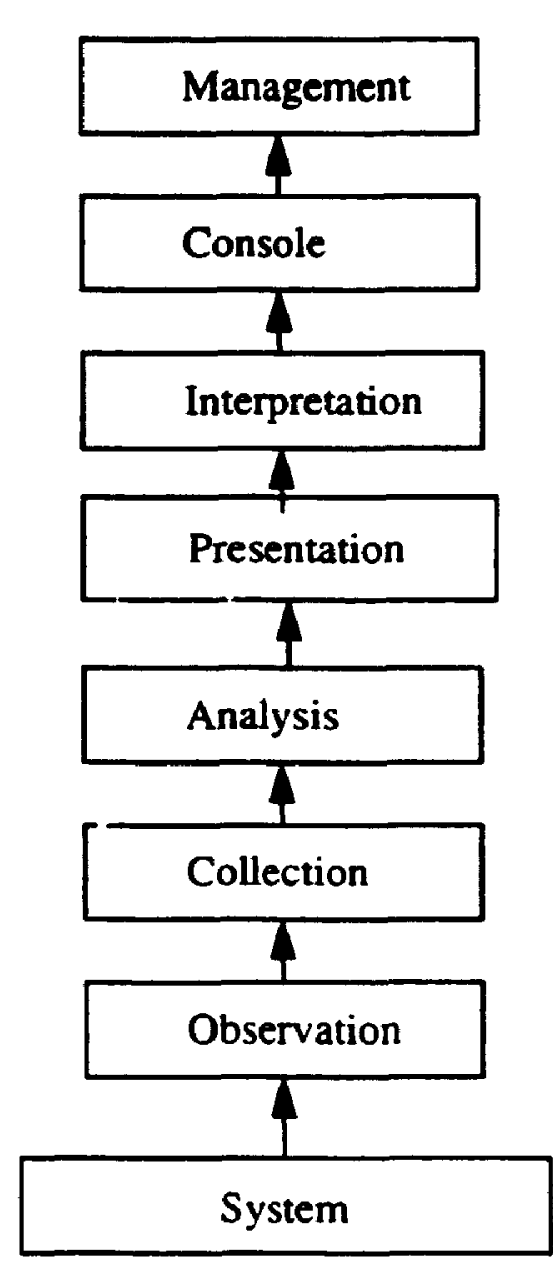

Figure 2.1 Layered view of a distributed-system monitor

\subsubsection{Program execution Monitors}

Program execution monitors, also known as program optimizers or program execution analyzers, are software monitors designed to observe application software. They help improve the performanre of the programs. Figure 2.2 shows the typical steps involved in program exe- 
cution monitoring. First, instrumentation are added to the target program. The instrumented programs then run under the control of the execution monitor. finally, the reports generated by the monitor are examined. Often the procedure is repeated several times and new instrumentation is added as more information about the execution profile of the program is obtained.

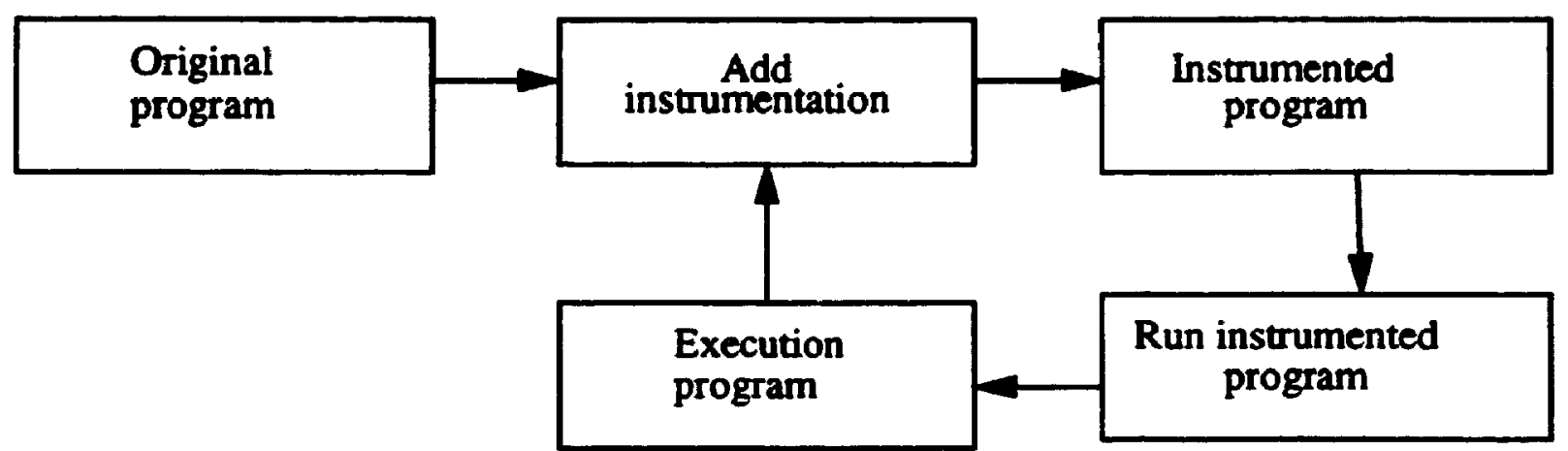

Figure 2.2 Steps in program execution monitoring.

Program execution monitors always have overhead so that it should use as less system resources as possible.

\subsubsection{Accounting logging}

Accounting logging is system monitor tool like UNIX system accounting facilities, for example, which monitors the processes $\mathrm{c} n$ the system. Some of performance data are reported, such as cpu time, disk $\mathrm{I} / \mathrm{O}$ and memory paging. Accounting logging is classified as a software monitor because it provides useful information about system usage and performance.

The main advantage of accounting logging is that they are built in the system. No extra effort is required to develop them. The overhead is generally small, so data collection can be performed for long periods of time. Further, the data reflects real-system usage.

The main disadvantage of accounting logging is that the log analysis programs can't provide 
with much detailed information. Most systems convert the log into a generally readable format or produce an overall summary. Additional analysis programs have to be written by the analysts themselves. The data in the logging may not be at the desired level of granularity. So it may not give enough information in detail. The accuracy of logging is also low. And it contains no system-level information, such as queue lengths or device utilizations.

\subsection{Workload Characterization and User Behavior Graph}

Measurement is always used with a particular workload. In order to perform meaningful measurements, the workload should be similar to the real workload in which the system works. Therefore, the workload characterization or modelling is one of the most crucial aspects of any performance evaluation project. No system evaluation study can avoid confronting the problem of modeling or at least selecting one or more workload. All performance analysis techniques, including simulation and measurement, require one or more workload model to be built.

In an interactive system, the workload mostly are from users, the interactive workload. It is heavily influenced by users' characteristics: think time, type time, user generated interrupts, and so on. This requires that the workload characterization includes the users' behavior. Therefore, the modelling of workload with users' behavior is needed. The typical workload is the user shell session. It begins with login command and ends with logout command. In a user shell session, the user issues different command type interactively [14]. There could be many workload components. Usually, we choose those workload components which are most used.

\subsubsection{Workload Characterization}

The major goal of workload characterization is to obtain a artificial workload for the system performance analysis. The general procedure of workload characterization design is shown in Figure 2.3 [13] [15]. 


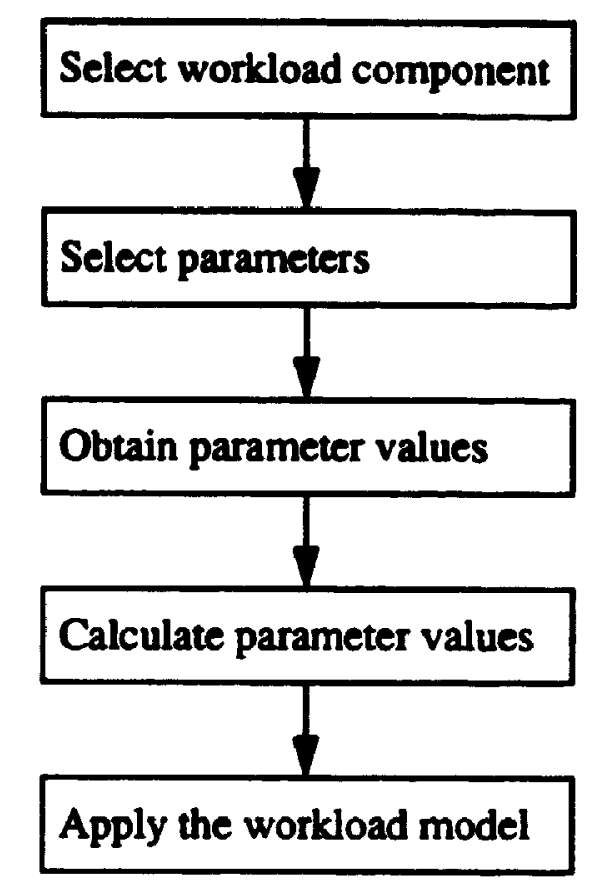

Figure 2.3. Procedure of workload characterization

The procedure of workload characterization in Figure 2.3 includes 5 steps:

(1). Select the workload component to be characterized. Common component types are the job, the command or interaction, the transaction, and the job step. The selection of workload component depends on the purpose of the study of the system. For the purpose of comparing different systems, some types of test workload can be used like Addition instruction, Instruction mixes, Kernels, Synthetic programs and Application benchmarks [11]. However, if we want to evaluate the system performance with a particular application, this application is the major workload component. For example, some machine only run one application like the library catalog service.

(2). Select the parameters which will be used to characterize the workload component. These parameters are the hardware and software resource demands of the component. Examples of the resources are CPU's, main memory, 1/O channels and devices, communication 
networks, terminals, and other peripherals. Logical parameters may be also needed, i.e, transition probabilities among the users actions, the user thinking time.

(3). Obtain values for each of these parameters by measuring a real workload. Typically, data is collected over a period of several weeks, often by monitoring as discussed in section 2.1 .

(4). Calculate values for these parameters by statistical analysis of collected data. Calculate the distribution and range of each parameter and transform or scale them to get data suitable for comparison. At this point, values outside the common range are discarded. The statistical techniques which are applied to the data include sampling, clustering, average, histogram and Markov modelling [11]. At this point, the workload model is obtained.

(5). Apply the characterized model, or some of its components, into the system performance analysis techniques, analytic modelling, for example.

Using the above workload characterization, the workload model can be built. An analyst can construct a synthetic workload representative of the actual system. There are several workload characterization techniques[11].

\subsubsection{User Behavior Graph}

The design of executable, artificial workload models is a preliminary fundamental step for all studies dealing with the performance evaluation of cornputer systems. The workload of an interactive system can be adequately described by a User Behavior Graph [15], as shown in Figure 2.4. The User Behavior Graph consists of a set of nodes, or states, and arcs. The states represent different commands issued by a user. When a command is chosen, it move from one state to another, and it remains in the state until the command is finished. The arcs represent the choice of the next command upon the completion of the current one. It means both the finishing of the previous state and the beginning of the new state with a new command. Similar, a Mosaic session could be described by a User Behavior Graph model. In a User Behav- 
ior Graph of Mosaic session, the nodes could represent the different selections of mouseoriented buttons or key-oriented commands. The arcs of the user graph are the next buttons or commands chosen upon the completion of the current one.

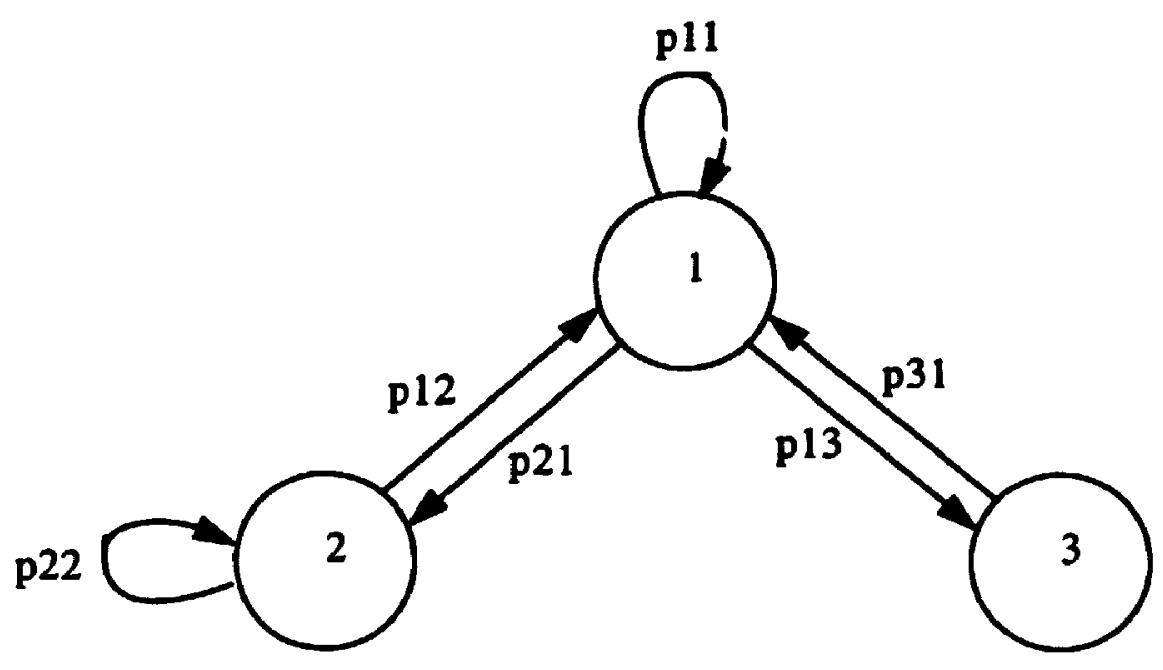

Figure 2.4. Example of User Behavior Graph with three nodes

The workload characterization includes static characterization which specifies the resource consumption of states, and dynamic characterization which specifies the changing of the workload [14]. Clearly, a User Behavior Graph can be modeled as a discrete time Markov chain with the transition probability matrix $P=\left[p_{i j}\right]$, so that the dynamic workload characterization of a interactive session can be performed. The Markov chain model could be aggregated or clustered based on the functions of the states or the resource consumed by the states. The resource based method of aggregation is more accurate than function based method [14]. Furthermore, by measuring the system resource utilizations in every state of the User Behavior Graph, the static characterization can be performed. Some techniques of classifying, reaggregating and clustering can be employed in both of functional and resource levels.

The User Behavior Graph in Figure 2.4 also provides useful an detailed information on the user behavior for the further software development. Some of the information may be not used 
in some system analysis techniques. For example, the static workload characterization may be enough for a performance analytic model. On the other hand, the dyramic workload characterization is useful for software developers to analyze and improve the software.

\subsection{System Performance Modelling}

Analytic modelling is one of the important techniques. Comparing to the measurement and simulation, analytic modelling requires the least time and the least cost. Usually, an analytic model is employed with another technique to evaluate the system performance. Many analytic models exist for system performance analysis. Some of them are summarized in [17] [18] [19].

Queueing Network Model (QNM) is a powerful and popular analytical model for evaluating system performance [20] [21] [11]. A Queue Network (QN) is a collection of service centers representing system resources, and customs representing users or jobs or transactions. Each service center is a queue on which the customs are either in service or waiting to be served. A job departing from one queue arrives to another queue (or possibly the same queue). Queuing network models are classified as either closed queueing network or open queueing networks. An open queueing network has external arrivals and departures. The number of jobs in the system varies with time. A closed queueing network has no external arrivals or departures. The jobs in the system keep circulating from one queue to the next. The total number of jobs in the system is constant. Figure 2.5 shows a typical closed network model of a computer system, in which the terminals are the jobs to be served, and the cpu and disks are queues. 


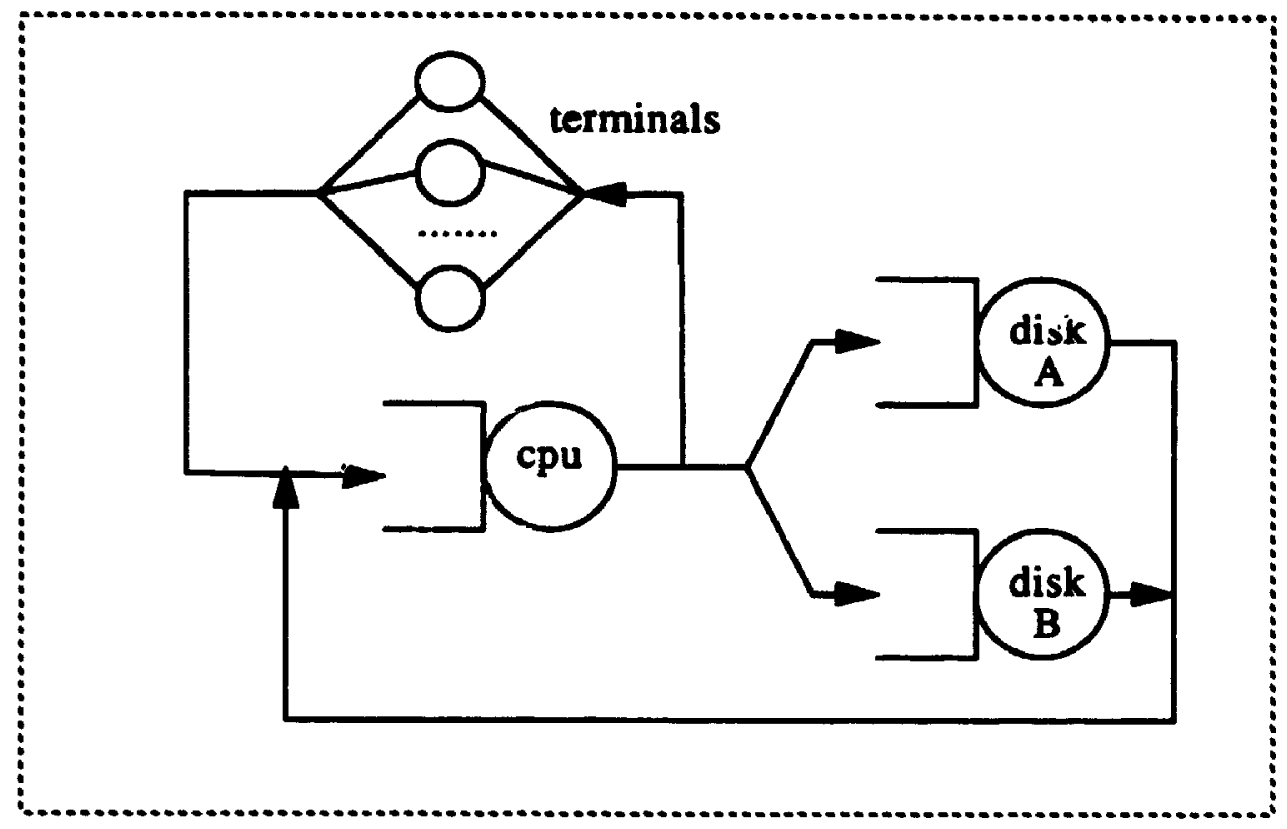

Figure 2.5 Closed Queueing Network Model

The queuing network could be either product form [11] or non product form. There are powerful and efficient techniques to solve product form model [17][21][11][22]. Mean-Value Analysis (MVA) [22], for example, is a exact solution. The approximating methods are needed for non product form QNs. Surrogate delay [23], for example, is one of the approximating methods.

The QNM is widely used in performance analysis of computer systems because it has many advantages. It provides both the modelling power and efficient solutions. It is easy to build, highly abstract and requires minimum of information as parameters. QNM also has its limitations of representing synchronization directly and in modelling parallel software for distributed systems. The WWW servers and their disks are queue service centers in the internet. Therefore, the WWW system could be modeled as QNM.

Another popular used modelling method is the Petri-Net Model [24][25]. Petri-Net contains a set of places, a number of tokens that reside at the places, and a set of transitions that repre- 
sent events causing tokens to move from one place to another following firing of transitions. With its advantages, It is promising tool for describing and studying information processing systems that are characterized as being concurrent, asynchronous, distributed, parallel, nondeterministic, and/or stochastic. Therefore, petri-net is a powerfu! modelling technique. But petri-nets have a weakness in modeling contention for resources and its low level of abstraction capability. It has no construct to represent queues and scheduling policies. The exact analysis in petri-net has a limitation on small models.

There are other techniques introduced in the past years. Stochastic Rendezvous Network Model (SRVN) [26] [27] [28] is a model of multi-tasking parallel software inter-task communication via rendezvous. Three View Models [29] describes performance modelling of concurrent software. Software Performance Engineering (SPE) [30] presents a systematic way to analyze the performance of software systems during their development process.

\subsection{Measurement And Performance Research with WWW applications}

Many measurement studies have been done on WWW applications. Some of them focus on the server side, others focus on the client/user side. For example, $\mathrm{H}$. Braun and $\mathrm{K}$. Claff [31], J. Sedayao [32], Lara D. Catiedge and J.E.Pitkow [33] have done measurement with WWW applications.

J. Braun [31] measured the queries to the popular NCSA Mosaic server to assess the geographic distribution of transaction request. They examine the WWW access log files for the NCSA Mosaic server, depict a time series of bandwidth and transaction demands, and break down the transaction load by geographic zones. They analyze the impact of caching the results of queries from the same zones, in terms of reduction of transactions and bandwidth from the main server, and discuss implications for cache maintainer regarding optimal timeouts or cache configurations. Clearly, their study is about the Workload characterization of the WWW server, the measurement of network traffic impact of WWW requests, and how to refine to web architecture using according to their measurement data. This study is mostly useful for the server design and improvement. 
J. Sedayao's paper [32] studied the typical network effects of Mosaic users, and how to minimize any negative affects and still give users good performance. Through examination of packet traces, analysis of $\log$ files, and simulation of an HTTP proxy server, they discover the effects of running Mosaic. First, they attempt to construct a network profile of an "average" Mosaic user at Intel. They take a look at the characteristics of common HTTP operations and the sizes of URLs that users retrieve. With this information in hand, they recommend some ways to improve performance and minimize the resource demands of network resources. We can see from the paper that the measurement focus on the server side, although the Mosaic is studied as it mentioned.

Both papers above study and analyze the performance of the network traffic of WWW applications. Both of them discuss the improvement of WWW applications. In other words, there are no much information about the user behavior.

Catledge and Pitkow's study [33] has more information about the user behaviors. Their paper presents the results of a study conducted at Georgia Institute of Technology that captured client-side user events of NCSA's XMosaic. Actual user behavior, as determined from clientside log file analysis, supplemented their understanding of user navigation strategies as well as provided real interface usage data. The methodology they used is to capture all events generated by consenting Georgia Institute of Technology's College of Computing staff, faculty and student populations who operate NCSA's XMosaic running Sun OS 4.1.3. Towards this end, a version of XMosaic was coded to trap all user interface level events. The analysis of the results is the user behavior which includes the methods of interaction, the site navigation, site analysis and directions for design. This is the most detailed paper in measuring and analyzing the client site of WWW applications. It gives the most realizable information of user behavior.

J. Pitkow' WWW user surveys [34] [35] give the general information of the user behavior, or the characterization of the WWW user. Different from [33] which measures a local system and give more detail of user behavior, these $s$ - reys give the global view of user behavior 
without measurement. Since the limitation of responses of users, the survey may just reflect those users who use WWW application frequently and skilfully. For example, the survey [34] show that $50 \%$ of the users have authored over ten HTML documents and only $7 \%$ have never authored a HTML document. It might be the case that most users who never authored a HTML document did not respond the survey. Therefore, more detailed and reliable characterization of WWW users is needed.

\subsection{Summary}

A brief review of the background related to this thesis is described in this chapter. Their review focuses on the measurement and analytic modelling since they are used in thesis. Measurement with different monitors are introduced. The most interested monitor for us is software, program execution and event-driven monitor. In several analytic modelling techniques introduced in this chapter, Queuing Network Model has its advantage for the thesis project. Workload characterization is both a part of the measurement evaluation and a part of performance modelling. Some of techniques of workload characterization is explained. The User Behavior Graph is introduced, not only because it is a good workload characterization model, but also because it can provide much detailed information for the software developer to analysis and improve the software. Finally, measurement and Performance research with $W W W$ applications are summarized. There was no performance modeling with $W W W$. It is a new research project for us. 


\section{Chapter 3}

\section{Measurement of Mosaic}

The measurement with both Lynx and Mosaic have been done during this study. Lynx is a typical text window based client, and Mosaic is a typical graphic window based program. Because most users use graphic window based clients like Mosaic and Netscape, we only include Mosaic as the case in the thesis. In this chapter, the measurement of Mosaic is explained. First, we describe the techniques used in the measurement, which is mainly instrumentation. Then, our experimental version of Mosaic with logging data will be introduced. Finally, the logging data will be explained.

\subsection{The Design and Techniques of Measurement}

The papers on measurements research of the WWW, as mentioned in last chapter, focus on different issues like server side, client side, traffic impact, user behavior, and so on. The methodology are also different, as for example, analyzing the logging file of server, measuring the events of client, or doing user survey. The methodology we used in our research is also different from other papers. Figure 3.1 shows the design of the measurement of WWW applications. The measurement of Mosaic client and of the WWW server are shown in Figure 3.1 (a) and 3.1 (b) respectively.

The measurement tool used in the server side includes the system accounting tool and built-in logging functions in the original program. In order to monitor the server process, the accounting tool has to keep trace of the process from start to end. In other words, the accounting tool starts first on the machine on which the server will run, and then the server starts. After the time of running for the measurements, the server process has to be stopped. At this point, the accounting logging data is recorded. Other logging data are saved in logging file through the built logging functions. Later on, the logging data are reduced for the use in WWW perfor- 
mance model. This performance model and more detailed explanation of measurement of the server side will be dealt with in chapter 6 . This chapter mainly focuses on the client side.

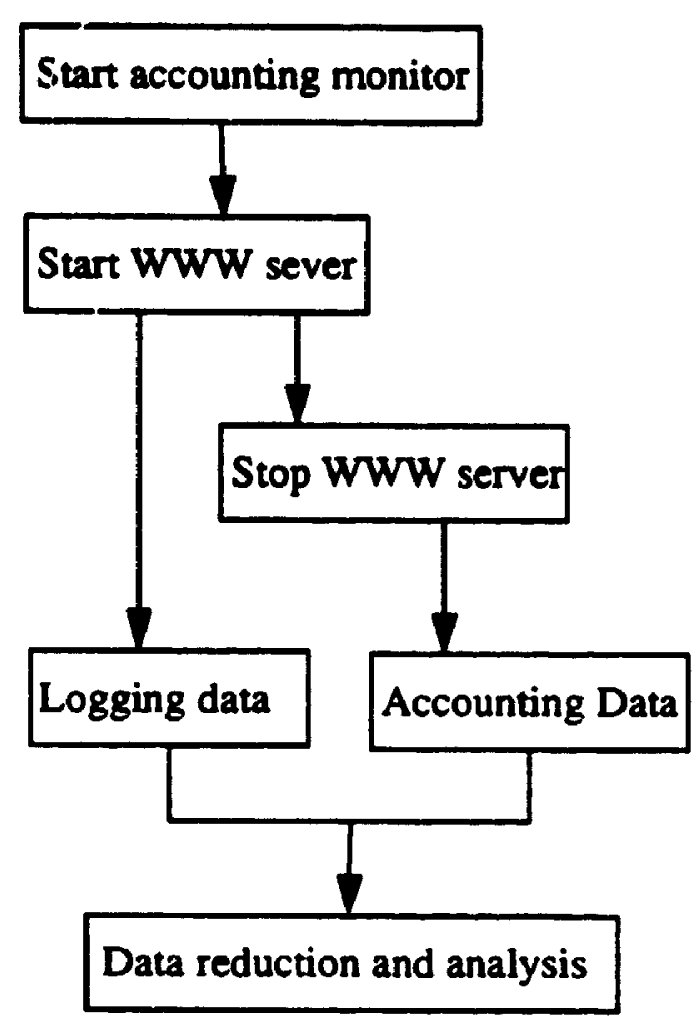

(a). Measurement of WWW server

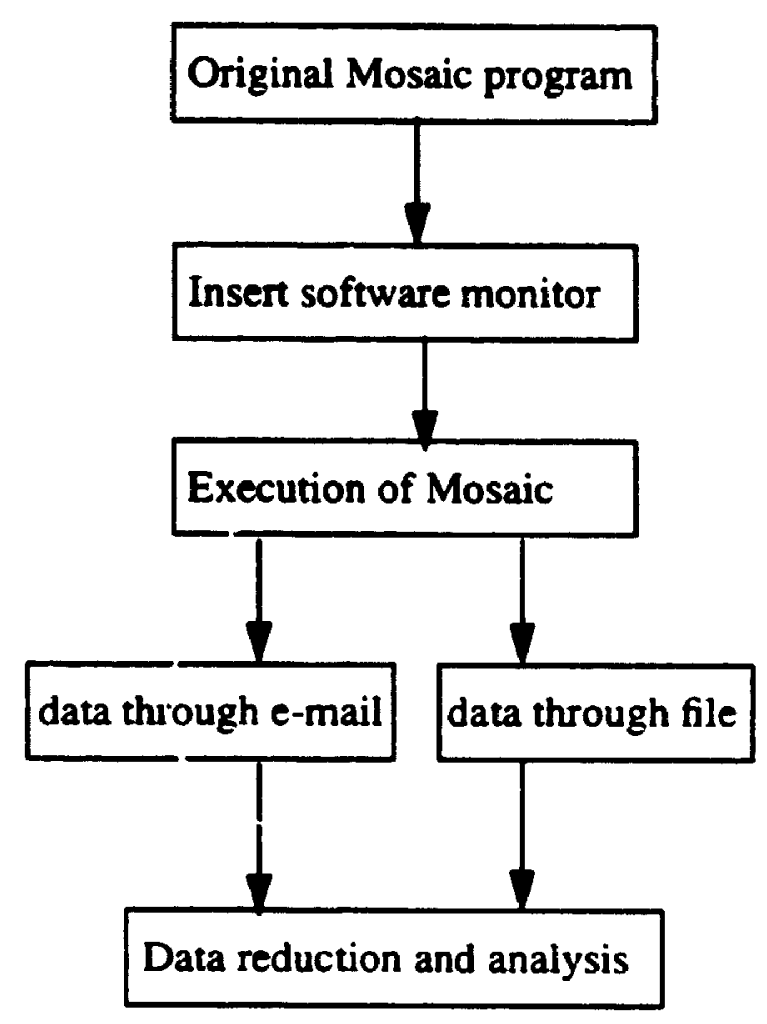

(b). Measurement of Mosaic

Figure 3.1 Measurement design of WWW applications

In a system which does not run WWW server or which has only an infrequently requested server, the impact of using $W W W$ is mostly from the client side. For this reason, more measurement is in the client side, the user behavior. the workload characterization of Mosaic client. The methodology is to use a software, program execution, event-driven monitor. First. instrumentation was installed in the original Mosaic source code, which is similar to [33]. We tried to capture most of user events in the client. This experimental version of Mosaic can be either set in the system as system application or just run by user as a user applications. The date measured can be collected either through e-mail or saved in a logging file. After gathering the data, new data reduction programs and some system tools are used to reduce the data and get the statistical results. With these measurement data, the User Behavior Graph [19] 
will be build. This is an Markov chain model of WWW client which will be solved. This is the dynamic workload characterization. In addition, the system resource demand of every state is also measured, so that statistical workload characterization will be obtained. That is, we will get $\mathrm{t}$ : - ystem resource demands of every state of the model. Also, the network traffic made by $W W W$ users are measured. This gives a complete description of a user's behavior and system impact.

In the User Behavior Graph model, some transitions and resource parameters depend on other parameters. For example, the probabilities of being 'active anchor' may be related to the number of anchors in current document. The time of reading a document is related to the size of the document. Hence, the information about the current document in the main window are also measured.

\subsection{Implementation of the experimental version of Mosaic}

As mentioned, we need a new version of Mosaic to monitor and capture the events and other measurement parameters. The monitor with the new version of Mosaic is a typical software, event-driven and program execution monitor.

The major and also the hardest work of the research project was the understanding of the source code of Mosaic, since there is no documentation with the source code. This includes the extent and functionality of the user interface, the communication, the processing and presentation of the hyper document. In the other word, we need to understand most of the source code of Mosaic which we need to modify. The source code also includes the source code of the CERN World-Wide Web Library. We should have the ability of $X$ window programming, UNIX communication programming and other programming techniques in order to instrument them.

For the convenience of study, I have installed my own WWW server which is: http://sundial.sce.carleton.ca:2500. This was my home page for the research period. Our local network have another WWW server for System and Computer Engineering Department at Carleton 
University, which is: http://www.sce.carleton.ca. It is also used for our research.

The original source codes of Mosaic is Mosaic-2.4 from the National Center for Superconducting Applications (NCSA). It uses the CERN World-Wide Web Library of Common Code from The European Laboratory for Particle Physics. Mosaic uses X windows interface including Motif, Xlib and Xt.

To implement the experimental version of Mosaic, the source code was modified for the purpose of getting logging data. The rizain changes are in those files or functions which are related to users input, window callbacks, operations before and after loading file(s), hypertext information and WWW widgets. Some monitoring instructions were added in the source codes. For example, getrusage() is the major system function for measure the system workload parameters. Here is a example of the use of getrusage() in the program:

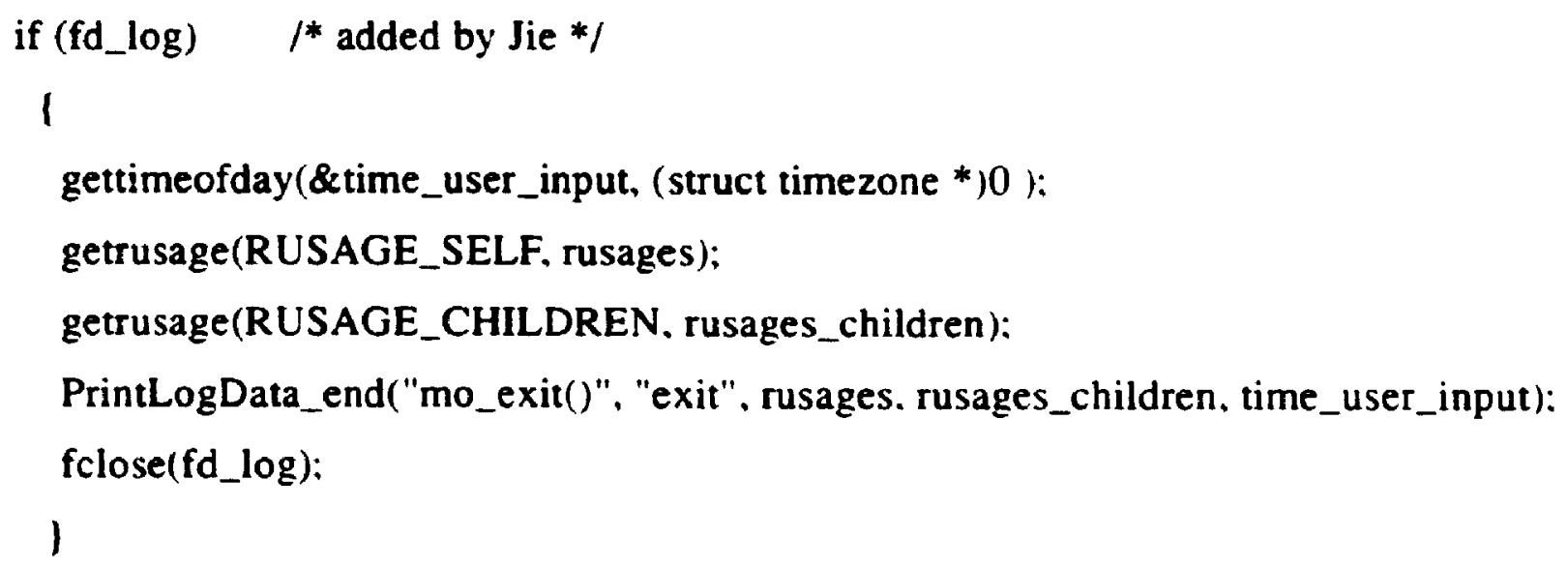

This segment of code is for monitoring the logging event "Exit" when the user exits Mosaic. The 4th line, getrusage(RUSAGE_SELF, rusages). is for obtaining the system resource consumed by the process from the start. The data are saved in the structure variable "rusages". The 5th line, getrusage(RUSAGE_CHILDREN, rusages_children). is for obtaining system resources consumed by all of its children processes from the start. The data are saved in the structure variable "rusages_children". The 6th line, PrintLogData_end("mo_exit()", "exit", rusages, rusages_children, time_user_inpur', is for saving the data into the logging file. PrintLogData_end is another function added for saving data. Most of logging events call it. The 
structure variable in which the data are stored gives the following system resources:

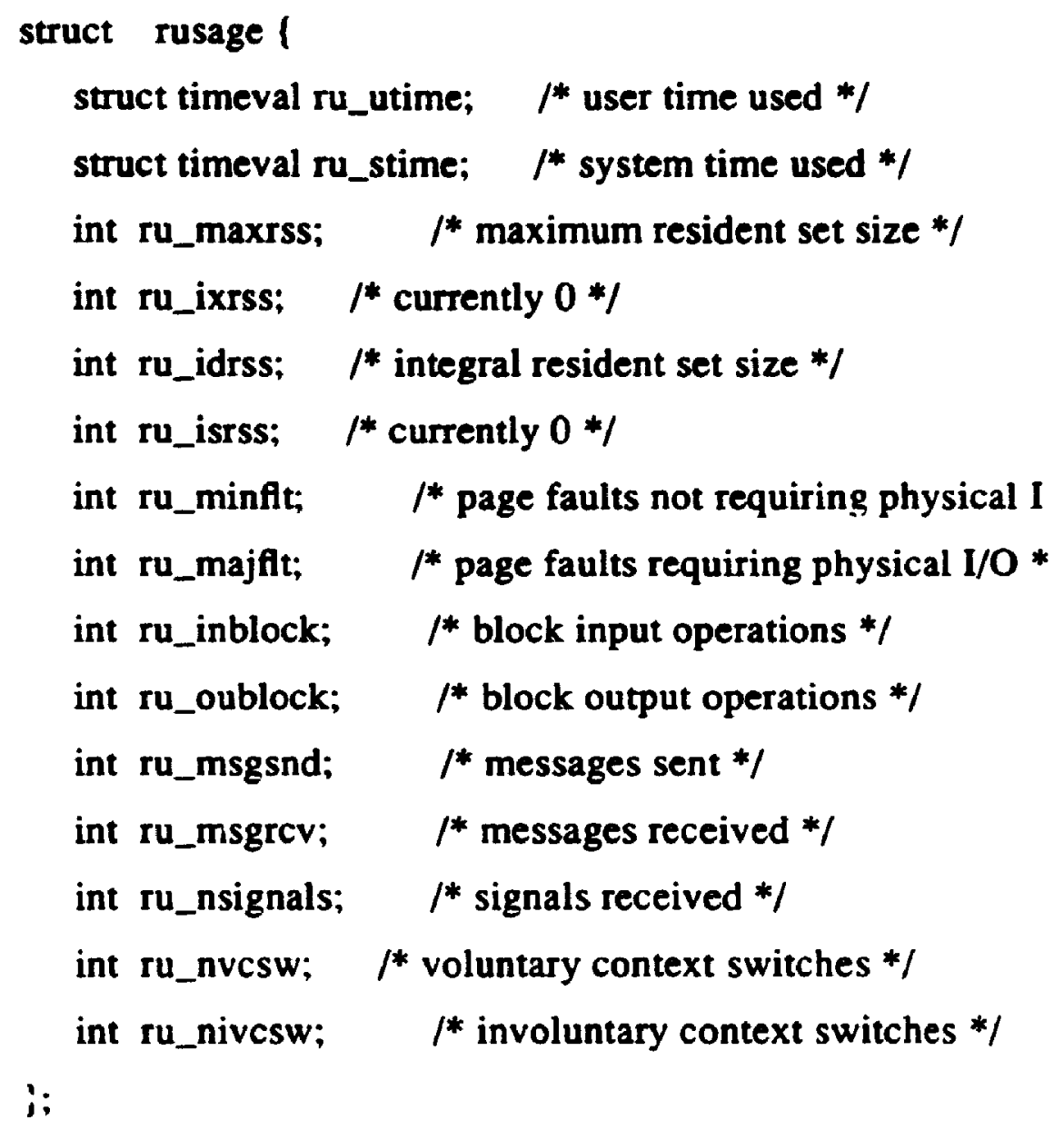

New functions for collecting data were added together with the monitor. One of them is to use an e-mail tool. The data are saved in a logging file in the user's home directory first. At the end of a session, the experimental version of Mosaic invokes the system e-mail tool to send the logging file to the author of this thesis. And the logging file in the user home directory is then deleted, and the program exits. The following source code is for implementing email function in the experimental version of Mosaic:

if (mail_log \&\& strcmp(log, mail_log) == 0)

I char system_command[256]; /* invoke system command */ sprintf(system_command,"mail jie@sce.carleton.ca < \%s\n", log); 
system(system_command); /* mail to jie the logging file */

sprintf(system_command, "rm -f \%sin", log);

system(system_command); $/ *$ delete the logging file */

1

I

exit (0);

In above code, the command "log" is the name of logging file which has the process ID as its suffix. The variable "system_command" stores the "mail" system command command first. After the logging file is sent to the author, it is replaced by the " $\mathrm{rm}$ " system command for deleting the logging file.

Another way to collect the data is just to save the data in a logging file. Some users prefer to invoke Mosaic (or Lynx) from the author's directory instead from the system, especially before and after the experimental version of Mosaic is installed in the system. In this case. some special files in the author's directory are open for the users. When users invoke Mosaic from the author's directory, the logging data are saved in these special logging files. This strategy is only useful for the local users. Another choice is to save the data in the user's own files without sending to the author, if the user prefer to do so.

The modified versions of Mosaic were built on SunOs and set in the local system with the system administrator's help. The uses of Mosaic were monitored whenever users invoked them, so that the measurement takes place in a real system environment.

The command lines of the experiment version of Mosaic are almost the same as the original one, except one more option is added. This additional option is for collecting logging data to a logging file. The format of the additional option is following:

Mosaic - $\log$ logfile 
Here, the 'logfile' is a file name in which the logging data will be put. Without providing the logfile option, the logging data will be sent to the author by e-mail, Most of logging data were collected through e-mail since users normally didn't use the option.

\subsection{Description of Logging Data in Mosaic}

In this section, we describe the logging data when Mosaic is running. Some of the data may be useful in the future study even though they were not used in the research in the thesis.

Following is a example of two events logging data script:

**********Mosaic_v4:Begining of a new session $* * * * * * * * * *$

Hostname: helicon.sce.carleton.caProcess ID: 1318

Host: helicon.sce.carleton.caPID: 1318

Begin_Loading_File: http://www.sce.carleton.ca/sce.html

Current_Tinie: 796691221.739171 seconds

ru_utime: 1.910000 ru_stime: 0.990000 seconds

ru_maxrss: 576 ru_idrss: 124763

ru_minflt: $290 \quad$ ru_majflt: 241

ru_nswap: 0 ru_inblock: 24

ru_oublock: 1 ru_msgsnd: 231

ru_msgrcv: 408 ru_nsignals: 0

ru_nvcsw: 504 ru_nivcsw: 443

Host: helicon.sce.carleton.caPID: 1318

Ended_Loading_File: http://www.sce.carleton.ca/sce.html

Bytes_loaded: 2610 Current_Time: 796691222.982054 sec'snds

ru_utime: 2.030000 ru_stime: 1.080000 seconds

ru_maxrss: 624 ru_idrss: 137662

ru_minflt: $301 \quad$ ru_majflt. 254 
ru_nswap: 0

ru_oublock: 1

ru_msgrcv: 436

ru_nvcsw: 540 ru_inblock: 24

ru_msgsnd: 271

ru_nsignals: 0

ru_nivcsw: 479

Success

There are many events and data in the logging file. To simplify them, we consider just 6 different events:

1). Begin_Loading_File: Just the beginning of loading a file.

2). Ended_Loading_File: Just the end of loading a file.

3. Begin_Loading_Image: Just the beginning of loading a image.

Many of the loaded files contain images which are separated from the text file. The loading of the images take place just after the completion of loading a text file in which the images are contained. Therefore, loading images is a different event from loading a text file. However, in the user behavior model, we will put the "loading file" and "loading image" together as one state as "Loading".

4). Ended_Loading_Image: Just the end of loading a image.

5). User_Input: Just after the user selection of any buttons or key commands.

In this event, there are many different user inputs. Some of them are rarely selected by users so that they are not included in our logging data, but 14 types of user inputs are monitored. Every user input is specified by a special field in the logging data.

6). Ended_User_Input: The end of the running user selection and just returning to main the main window 
In every Mosaic session, the first two lines of the logging data tell us that a new session of Mosaic begins to run, and give the host name on which Mosaic is running, and give the process ID. After the first two lines, all the data between two blank lines are from one logging event. Some of the logging data are the same in different events, and others are different:

All of the 6 events have the following data since the start of the program:

Host: $\quad$ The host name on which Mosaic is running;

PID: $\quad$ The process id of the running Mosaic session;

Current_Time:The current time (in seconds) since 00:00 GMT, January 1, 1970;

ru_utime: The total cpu time (in seconds) spent executing in user mode;

ru_stime: The total cpu time (in seconds) spent executing in system mode;

ru_maxrss: The maximum resident set size in pages ( $4 \mathrm{Kbytes} /$ page by default);

ru_idrss: Amount of memory in use by the process while it is running;

ru_minflt: The number of page faults serviced which didn't require any physical I/O activity;

ru_majflt: The number of page faults serviced which required physical $1 / O$ activity;

ru_nswap: The number of times the process was swapped out of main memory;

u_inblock: The number of times file system had to perform input in servicing a read request;

ru_oublock: The number of times file system had to perform output in servicing a write request;

ru_msgsnd: The number of messages sent over sockets;

ru_msgrcv: The number of messages received from sockets;

ru_nsignals: The number of signals delivered;

ru_nvcsw: The number of times a context switch resulted due to the process voluntarily giving up the processor before its time slice was completed;

ru_nivcsw: The number of times a context switch resulted due to a higher priority process becoming runnable or because the current process exceeded its time slice; 
Additionally, every event has its own logging data which are different from other events. Following are the logging data which are different in different events:

1). Begin_Loading_File:

Begin_Loading_File:The begging of loading a file. The URL of the file being loaded is followed;

2). Ended_Loading_File:

Ended_Loading_File:The end of loading a file, The URL of the file loaded is followed: Bytes_loaded: The file size of the loaded file;

Status: A string which is 'Success' or 'Failed', to indicate if the loading is success.

3. Begin_Loading_Image:

Begin_Loading_Image:The begging of loading a image. The URL of the image being loaded is followed;

4). Ended_Loading_Image:

Ended_Loading_Image:The end of loading a image. The URL of the image loaded is followed;

Bytes_loaded: The file size of the loaded image:

Status: A string which is 'Success' or 'Failed', to indicate if the loading is success.

5). User_Input: 
User_Input: The begging of user input. The name of the user selection is followed;

lineCount: The number of lines in the current presenied text;

anchorCount: The number of anchors in the current text;

activeAnchorPosition:The position of the active anchor in the whole document anchors;

\section{6). Ended_User_Input:}

Ended_User_Input The end of user input. The name of the user selection is followed;

\subsection{Summary}

In order to build the user behavior and the program model of Mosaic, a software, eventdriven and programming execution monitor is built for Mosaic. This is our experimental version of Mosaic. The implementation of the experimental version of Mosaic took the author's most time of the research. With this experimental virsion of Mosaic, we monitor the execution of Mosaic and gather the logging data for most user input events and program events either through e-mail or in logging files. There are 6 kind events in the logging data: Begin_Loading_File, Ended_Loading_File, Begin_Loading_Image, Ended_Loading_Image, User_Input and Ended_User_Input. The User_Input includes 14 different types of user inputs. In monitoring of the events, parameters and system utilizations are measured. 


\section{Chapter 4}

\section{User Behavior of Mosaic}

In this chapter, a user behavior model of Mosaic is built, which is a Markov chain model. The user inputs and program events are defined as states in the model. Then we measured the model using our logging data from experimental version of Mosaic. The transition probabilities is the major issue to be measured in this model, which representative the user behavior. The mean transition probabilities of the states the model are given.

\subsection{The Definition of User Behavior Graph of Mosaic: Mosaic Model}

Mosaic is an interactive application. Users make their own decision to choose different operation .. In Mosaic session, the different user selections lead to different state (refer to the User Behavior Graph in 2.2). We first define a term. user input type, in Mosaic. In Mosaic, a user can either click a widget button (i.e., 'Back'. 'Forward', 'Save As', 'Hotlist', etc.) or click a anchor in the hyper text window. All of user selected key commands, widget buttons and anchors are called User Input Types. A Mosaic session is similar to a shell session so that we can employ the same techniques of workload characterization described in section 2.2.2.

The User Behavior Graph of Mosaic is shown in Figure 4.1.There are 16 states in this model. Other states which occurred less than 4 times of 115 Mosaic sessions were ignored in the model. A action is completed in a state. Two events are needed for one state. One is for entering into the state. The other one is for exiting from the state. For example, the "saving" state represents the "saving file" action from the users' choice. When starting the "saving" function in the program, it enters to the "saving" state in the Mosaic model. When "saving" is finished, the program returns from the "saving" function, and it leaves the "saving" state. Table 4.1 gives the meaning of all the states in the model. 


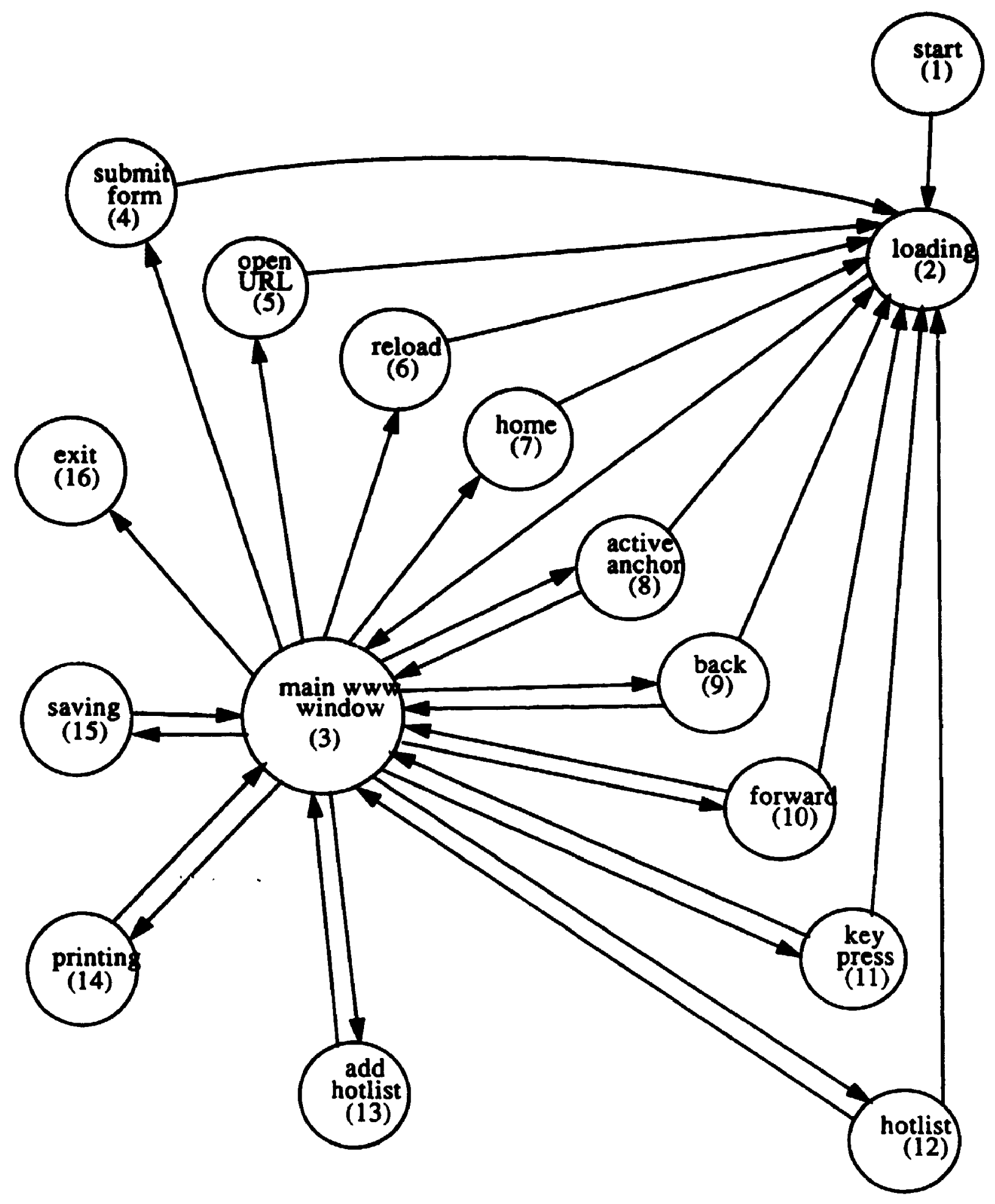

Figure 4.1 User Behavior Graph of Mosaic 
State

start

loading

main www window

submit form

open URL

reload

home

active anchor

back

forward

key press

hotlist

add hotlist

printing

saving

exit

\section{Meaning}

Start running the program until the beginning of loading the home page. Loading a file, from the start of loading to the end of loading The dormant state where the user reads document/thinks, from the completions of the previous action until choosing next action.

Action of sending a form message back to servers, e.g. "search", from start of submitting until the beginning of loading the result.

Open (or load) a document by providing the address, URL [36], manually instead of by pressing a button. It lasts from clicking the "open" button until beginning of loading the document.

Reload (or upgrade) the current document, from pushing the "reload" button until the beginning of load the document.

Go to the home page, from pushing the "home" button until the beginning of loading the home page document.

Choose a button which has a link to another document, from pushing a button in the document until the beginning of loading the new document linked to the button, or until returning to the "main window" (in this case, the document is already in the memory) Back to the previous document, from pushing the "back" button until the beginning of loading or until returning to "main window".

Go to the document from which the user backed last time. Starting by pushing the "forward" button until the beginning of loading document or until returning to the "main window"

A key command is pressed, which is handled by a special function. The state is entered by pressing a key in "main window" and left on loading a file or returning to the "main window"

A subwindow for maintaining the bookmark file, from selecting the hotlist window until loading a file or returning to "main window" add the current document URL to the bookmark file, from pushing the "add hotlist" button and return upon its completion.

A subwindow for printing the current document, from selecting the "printing" hutton until returning to the "main window"

A subwindow for saving the current document, from selecting the "saving" button until returning to the "main window"

Exit the program, from selecting the "exit program" button or "close", until the program exit.

Table 4.1 Meaning of the states in Mosaic model (Figure 4.1). 
Each state in the graph represents an interactive user input type, with the exception of state "loading" and "start". When a Mosaic session starts, it stays in "start" state for a while. After starting, the "loading" state follows since Mosaic loads the home page automatically. Whenever Mosaic leaves the state "loading", it enters the "main window" state, which is the "dormant node". In this state, the user read the text, looks at picture, scrolls the scroll bar and moves the mouse pointer. During the Mosaic session, the states are chosen following the arcs of the graph. Whenever the user choose a input type (e.g. pushes a button), Mosaic enter a new state. The "loading" state is a special state, which is chosen by Mosaic instead of users. For example, when in state 'open URL', the 'loading' would be the next state automatically. The choosing of the next state is based on the state transition probabilities.

From the model in Figure 4.1, we can see that most states are followed by only one or two other states, except the "main window" state. Not any state could go to the "start" state because it is the start of a new Mosaic session. When a session starts, it enter "start" state, and then go to "loading" to load a file which is the home page. Whenever a file is loaded, it always go to the "main window" state in which the user is reading/typing. From state "main window", it can go to most of other state except "start", "lor:ting" and "exit". The "exit" state is the end of the Mosaic session. It is only entered from "main window" state. Some state can only $g 0$ to one state upon its completion, and their transition probabilities to other states are known as 1 .

Another issue we should explain is that all the states don't have re-entry to themselves. This is because we integrated those self-reentry transition of a state inside the state. For example, in the "main window" state, the user can have more actions like reading, typing, moving mouse and scroll the scroll bars. But all of this actions is in the document (the same file loaded). Therefore we comprise them inside of the state.

The experimental version of Mosaic was installed on SunOS of our system for 29 days. There were total 115 sessions. We previewed the logging data and only selected those actions which occur frequently (more than 4 occurrences) as the states in the model of Figure 4.1. About 13 actions like 'refresh document' and 'mail document' never occurred. The rest of actions 
occurred only 4 times or less, like 'search' and 'open local document', and also are ignored. For the reason of simplifying the model, some other actions which happen inside Mosaic are included in other states. For example, the scroll bar operation and moving the mouse pointer are included in "main www window" state.

\subsection{Transition Probabilities of Mosaic Model}

A program "data_process" was written to process the logging data. It has the following functions:
1. Compress data file
2. Restore data file.
3. Raw report on compressed file
4. Detail report on compressed file
5. Quit

The function 4, "Detail report on compressed file", produces about 30 files as the input files of the Mathematica package. Mathematica programs read these files. and give the text and visual results.

Different statistical analysis results are obtained from the $C$ program and Mathematica programs. From all of the 115 sessions, the sate transition occurrences and state transition probabilities are obtained. Table 4.2 shows the transition occurrences of the Mosaic model defined in Figure 4.1. And Table 4.3 shows the transition probabilities of the model. For the convenience of view, Figure 4.2 show the Mosaic model again from Figure 4.1, but with the transition probabilities shown on it. The probabilities which are not shown in Figure 4.2 are unity. As we already saw from the definition of the User Behavior Gragh in Figure 4.1, most of the transition occurrences and transition probabilities are zero. The remaining transition probabilities are only meaningiul with states $3,8,9,10,11$ and 12 since the any of other states can only go to a definite state next.

As we mentioned that the "Main_Window" is a dormant state for the user. The user inputs are 
always come from "Main_Window", and transition always go back to "Main_Window" eventually, except the "Start" and "Exit" states. From the "Main_Window" state, mostly the user loads a new document (state 8 ) by pushing a button in the WWW document, which has about 0.45 of the nrobability. This means the users always like to find new information through the easy-linked buttons. Also, from Table A.1 and Table 5.2, we can prove that the most frequent state in Mosaic is "Loading" documents. And this is just the power of WWW system. The next most frequent chosen user selection is the "Back" button (state 9), which is about 0.3 of the probability. There is no surprise that the users like to explore the new documents before reading a the whole old one, and then come back to read it again. It is surprising that users rarely load files from the "hotlist" which is only about 0.03 of the probability. This might be because the most users are inexperienced. The logging data show that most of the users of the experimental version of Mosaic are students.

In Table 4.2, the "Surn" column is the accumulation of all the states occurrences. We can also see, from the table, that the most occurred states are "Man_Window", "Loading", "Active Anchor" and "Back". This also means the user use their most time to load and read document. either by getting a new docrment or backing to the old one.

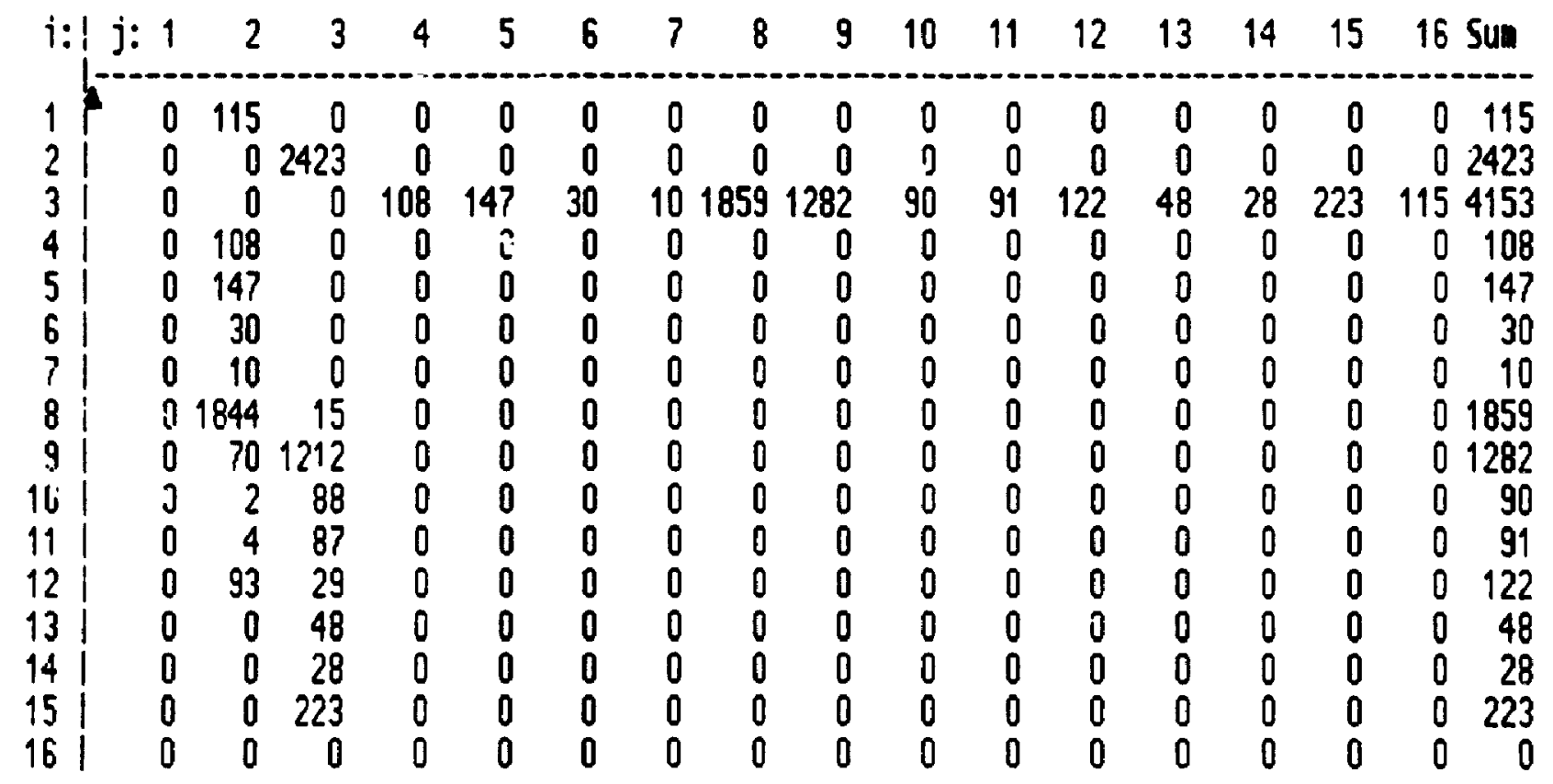

Table 4.2. Transition Occurrences From State i to state $\mathrm{j}$ 


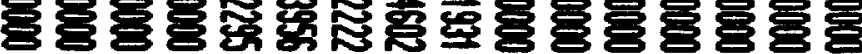

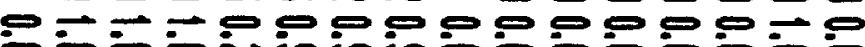

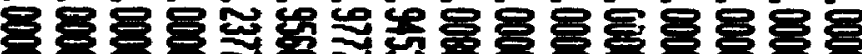

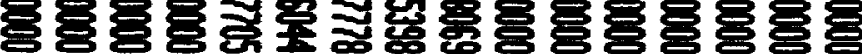

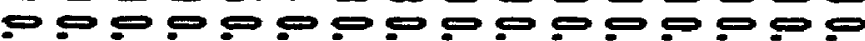

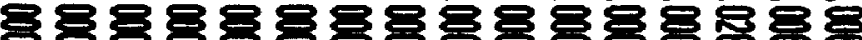

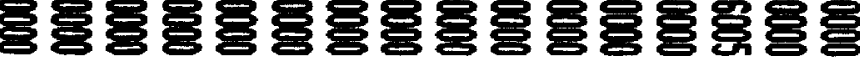

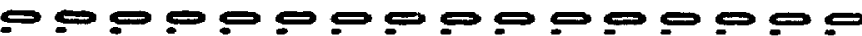

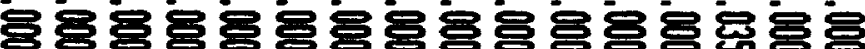

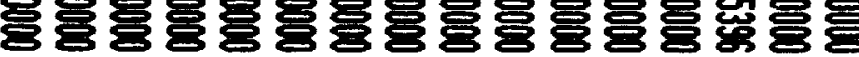

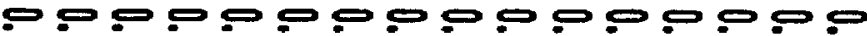

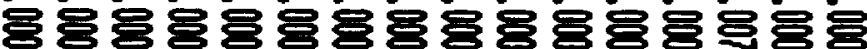

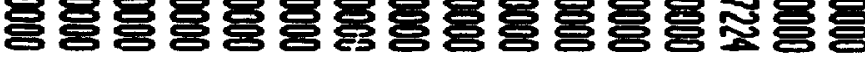

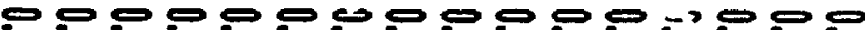

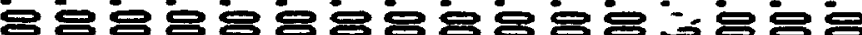

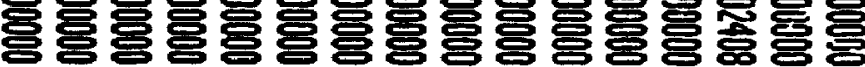

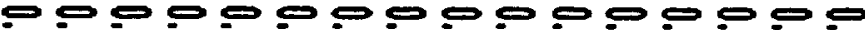

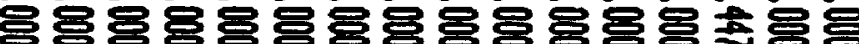

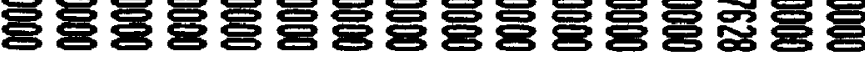

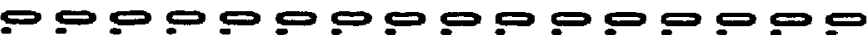

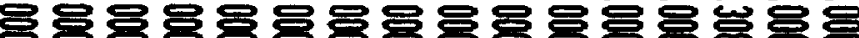

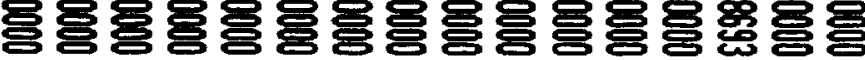

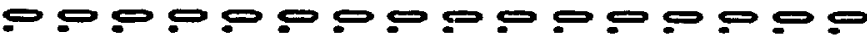

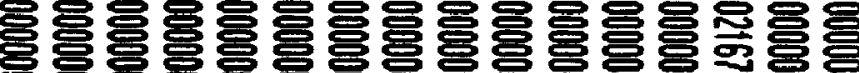

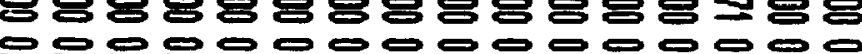

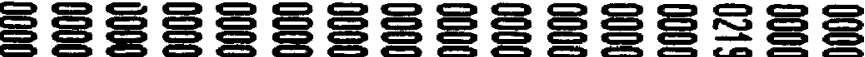

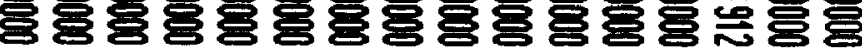

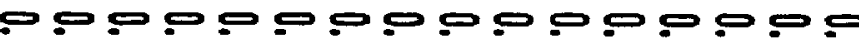

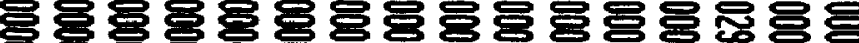

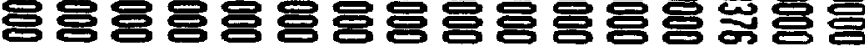

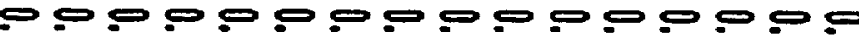

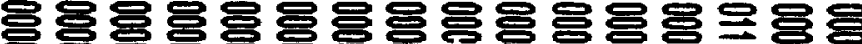

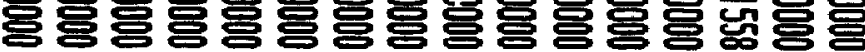

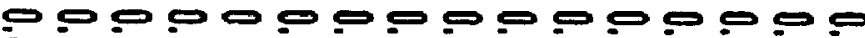

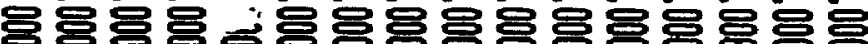

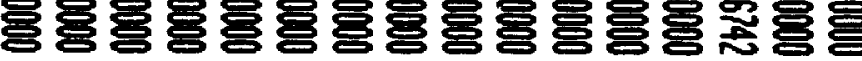

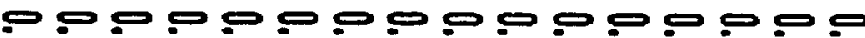

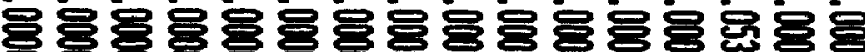

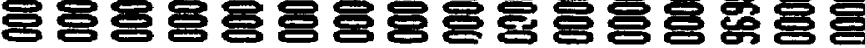

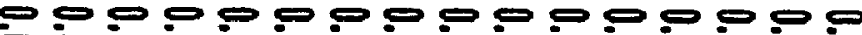

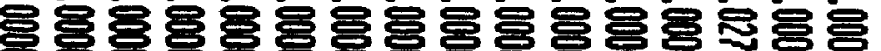

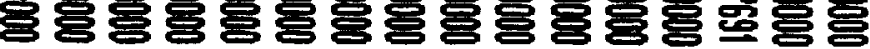

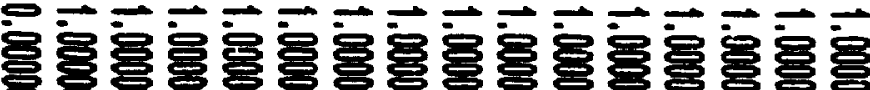
$\infty$
$\overrightarrow{0}$
$\overrightarrow{0}$
$\vec{\sigma}$
$\vec{\sigma}$

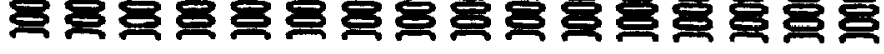




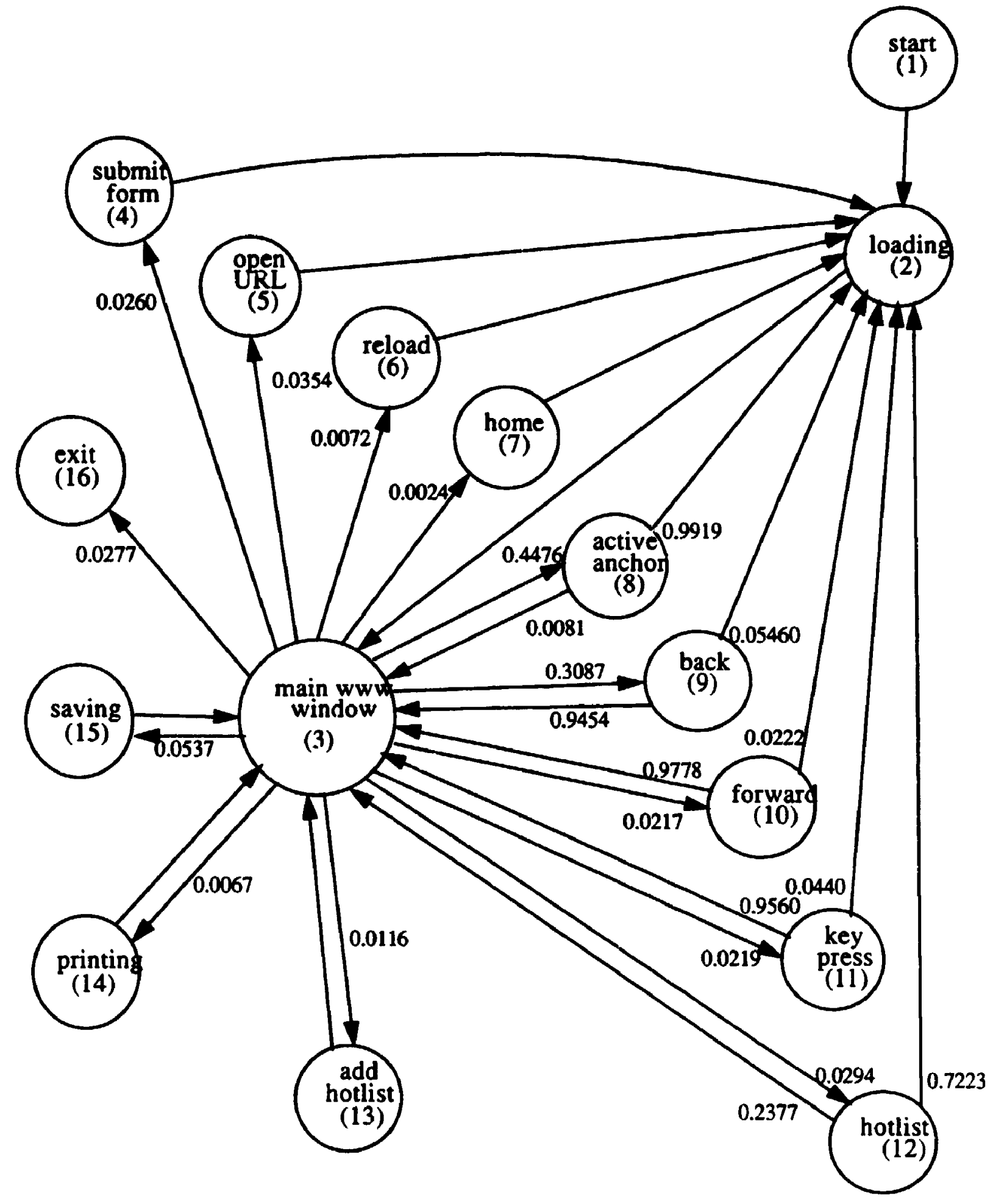

Figure 4.2 User Behavior Graph of Mosaic with transition probabilities.

The probabilities not shown are $1 \mathrm{~s}$. 
Some other statistical data are also obtained, e.g. the common state occurrences which are the number of occurrences of every state among all the logging data together, and the probabilities of the state occurrences among all of the state occurrence. These data are presented in Appendix A.

\subsection{Summary}

Mosaic is an interactive application which can be described with a User Behavior Graph. The actions either chosen by the users or invoked by the program itself are defined as the states the User Behavior Graph model, a Markov Chain model. Using the measured data from the experimental version of Mosaic, the transition probabilities of the model is obtained. It shows that the users mostly go and back to explore the informations. The model is not only useful for our performance research, but also the further understanding of the software. 


\section{Chapter 5}

\section{Parameters and Some of Metrics of Mosaic Model}

In chapter 4, we defined the User Behavior Graph of Mosaic, and we found the transition probabilities of the user model. We must also characterize the parameters of the Mosaic software when it executes an command. All the parameters and measurement metrics are given in term of the states of the model in Figure 4.1. They are represented by the measurement in one visit to a state, from the start of visiting until the exit of the state (refer to Figure 4.1 and Table 4.1).

In the measurement of Mosaic, we try to get as many parameters as possible for present and future analysis. We will emphasize on the most common and important parameters and metrics which we need for the research. Then all of the parameters measured will be described briefly. In the User Behavior Graph model, different states may have different parameters. For example, the file size loaded is a important parameter for "loading" state, but not for other states. Some of parameters used in the thesis are not system resources, but they are also important for some states. For example, the elapsed time in the "main window" state means the reading time in which the user read the current hypertext. The number of anchors in the "main window" is meaningful since the transition probabilities may be related to it.

\subsection{Cpu Time of Mosaic Model}

Because cpu time is the most important system resource and parameter, so that we pay more attention on the cpu time. A cpu time in a state is defined as the cpu time demand in one visit to the state, from entering until leaving the state. Table 5.1 shows the total cpu times spent in every state. These cpu time also described with means, variances, standard derivations and confidence intervals 


$\begin{array}{cllll}\text { State } & \text { Mean } & \text { Variance } & \text { SD } & 95 * \text { CI } \\ 1 & 2.1177 & 0.3244 & 0.5695 & \{1.9956,2.2398\} \\ 2 & 0.6115 & 14.5038 & 3.8084 & \{0.4537,0.7693\} \\ 3 & 0.1337 & 0.1195 & 0.3456 & \{0.1228,0.1446\} \\ 4 & 0.0051 & 0 . & 0.005 & \{0.004,0.0063\} \\ 5 & 0.1744 & 0.062 & 0.2491 & \{0.129,0.2199\} \\ 6 & 0.0037 & 0 . & 0.0055 & \{0.0019,0.0056\} \\ 7 & 0.0038 & 0 . & 0.0053 & \{0.0022,0.0054\} \\ 8 & 0.2198 & 0.2164 & 0.4652 & \{0.1982,0.2414\} \\ 9 & 0.169 & 0.0584 & 0.2416 & \{0.1559,0.1821\} \\ 10 & 0.1722 & 0.0568 & 0.2384 & \{0.1121,0.2322\} \\ 11 & 0.0808 & 0.0341 & 0.1847 & \{0.0441,0.1175\} \\ 12 & 0.2997 & 0.1962 & 0.443 & \{0.1451,0.4543\} \\ 13 & 0.0275 & 0.0002 & 0.0144 & \{0.0198,0.0352\} \\ 14 & 0.2098 & 0.0349 & 0.1867 & \{0.1589,0.2608\} \\ 15 & 0.3353 & 0.0684 & 0.2616 & \{0.2092,0.4613\} \\ 16 & 0.1599 & 0.0436 & 0.2089 & \{0.1101,0.2097\}\end{array}$

Table 5.1. Cpu times per visit of states in Mosaic model (seconds)

It may be worth to look at the distributions of cpu times. From our statistical analys; distributions of the cpu times are closed to exponential distributions, although they are not exponential distribution, according to the Chi-Square test. According to our data statistics. the more logging data was obtained, the closer it was to an exponential distribution. An example of thic cpu time distributions, which is the "main window" state, is shown in Figure 5.1 which is a histogram. The horizontal axis is the number of cells of the sample data of $\mathrm{cpu}$ time from minimum to maximum. The vertical axis is the number of occurrences of the sample data. The number of cells depends on the range of the cpu time. More cells are for large range of cpu time. The pair of number at the right end of horizontal axis is the range of cpu time. We can see from Figure 5.1 that its distribution is close to exponential. All of the other cpu time distributions are similar, and are shown in Appendix B. 


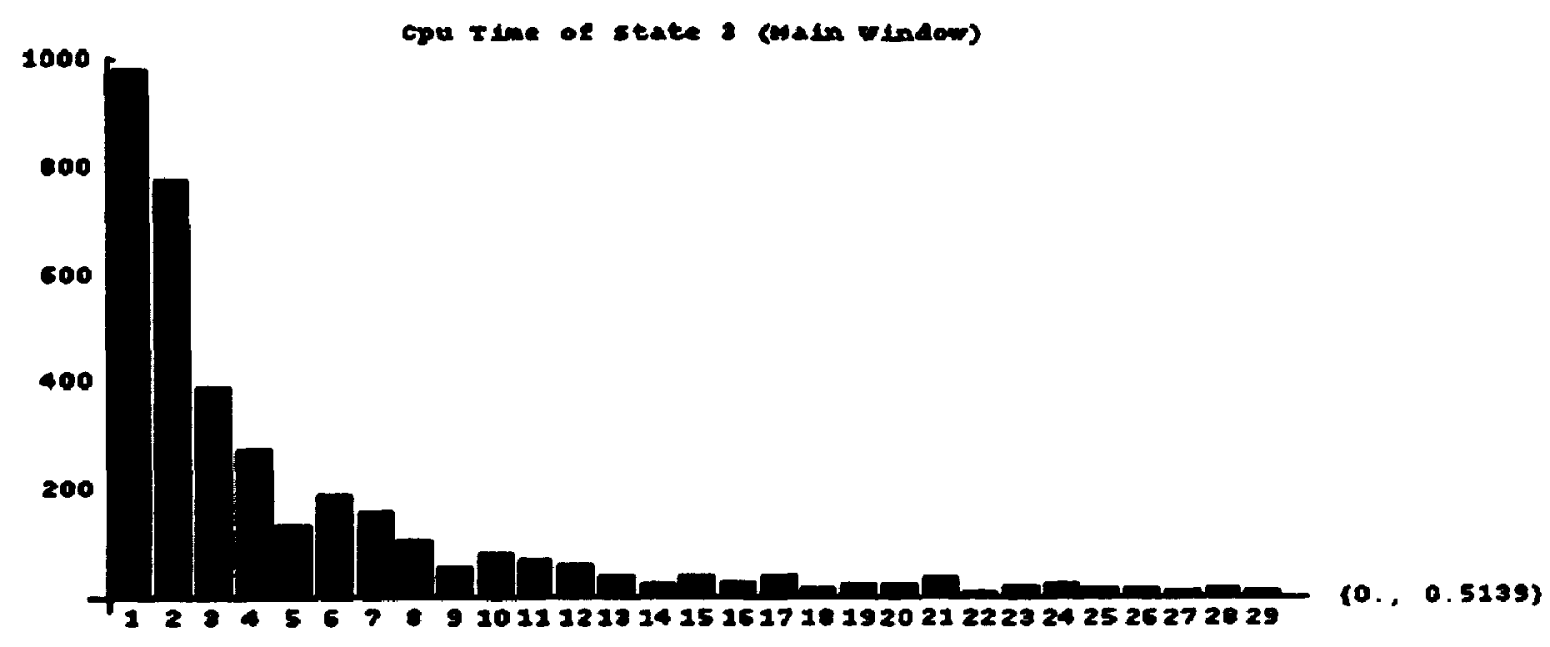

Figure 5.1. Cpu Time Distribution of State 3 (main window), from 0 to 0.5139 Seconds into 29 cells

No. State Name Cpu/visit Rank Occurred Cpu_total Rank2 Percentage

$\begin{array}{lllllrlr}1 & \text { Start } & 2.1177 & 1 & 115 & 243.5344 & 4 & 7.08 \% \\ 2 & \text { Loading } & 0.6115 & 2 & 2423 & 1481.6645 & 1 & 43.09 \% \\ 3 & \text { Main Window } & 0.1337 & 11 & 4153 & 472.1961 & 2 & 13.73 \% \\ 4 & \text { Submit Format } & 0.0051 & 14 & 108 & 0.5508 & 14 & 0.02 \% \\ 5 & \text { Open URL } & 0.1744 & 7 & 147 & 30.3456 & 9 & 0.88 \% \\ 6 & \text { Reload } & 0.0037 & 16 & 30 & 0.1110 & 15 & 0.00 \% \\ 7 & \text { Home } & 0.0038 & 15 & 10 & 0.0380 & 16 & 0.00 \% \\ 8 & \text { Active Anchor } & 0.2197 & 5 & 1859 & 407.1041 & 3 & 11.84 \% \\ 9 & \text { Back } & 0.1690 & 9 & 1282 & 216.6580 & 5 & 6.30 \% \\ 10 & \text { Forward } & 0.1722 & 8 & 90 & 15.4980 & 11 & 0.45 \% \\ 11 & \text { Key Press } & 0.0808 & 12 & 91 & 73.5280 & 7 & 2.14 \% \\ 12 & \text { Hotlist } & 0.2997 & 4 & 122 & 36.5634 & 8 & 1.06 \% \\ 13 & \text { Add Hotlist } & 0.0275 & 13 & 48 & 1.3200 & 13 & 0.04 \% \\ 14 & \text { Printing } & 0.2098 & 6 & 28 & 5.8744 & 12 & 0.17 \% \\ 15 & \text { Saving } & 0.3355 & 3 & 223 & 74.8165 & 6 & 2.18 \% \\ 16 & \text { Exit } & 0.1599 & 10 & 115 & 18.3885 & 10 & 0.53 \%\end{array}$

Table 5.2 Rank of states according to the mean cpu time, from high to low. 
Table 5.2 shows the rank of state with the cpu time consumed from high to low. The meaning of the columns are following:

No. The number of the state as shown in Figure 5.1

State Name The name of the state as shown in Figure 5.1

Cpu/state Mean cpu time of a state in one occurrence.

Rank The rank of the state with the cpu time in one occurrence

Occurred Total occurrences of state in our measurement as shown in Table A.1.

Cpu_total Total cpu time of state in our measurement

Rank2 The rank of state with the total cpu time in our measurement

Percentage The percentage of the cpu time of a state to the total cpu time

We can see, from the table, that the "Start" start consumed the most cpu time among the state. This is the initialization of Mosaic, including loading the program to the machine. However, this state only occurred once for a session so that its rank is reduced to number 4 according to the total cpu time consumed during the measurement period.

State "Loading" is the second most cpu consumed state. However, it is the number one cpu consumed state according to the total cpu time. The percentage of cpu time of "Loading" state is $43.09 \%$ of the cpu time consumed. This is because the this state is the most occurred state.

"Main Window" and "Active Anchor" states is the number 2 and number 3 total cpu consumed respectively. It is also because they occurs frequently.

Some states like "Home", "Reload", "Submit Form" and "Add Hotlist" only take very little cpu time from the total cpu time. So the performance of Mosaic wouldn't affected by these state. 


\subsection{File Size in Loading State}

The file size of the document loaded is another important parameter. Many of other parameters or performance metrics depend on how big is the document fle. For example, the bigger the file, the bigger delay of loading. The bigger the file, the more demand on the server per request.

The file sizes of the documents in the internet are very different. The biggest file which the author loaded is more than $14 \mathrm{Mb}$. The biggest type of files normally are audio files. The image files are the second biggest type of files. Text file are normally small. According to our measurement, the mean file size had been loaded from Mosaic is $\mathbf{2 7 5 6 1}$ bytes with standard derivation 322826. And the confidence interval is $\{14185,40936\}$. The files counted are the new file loaded, not including those in cache.

The distribution of the file size is shown in Figure 5.2.

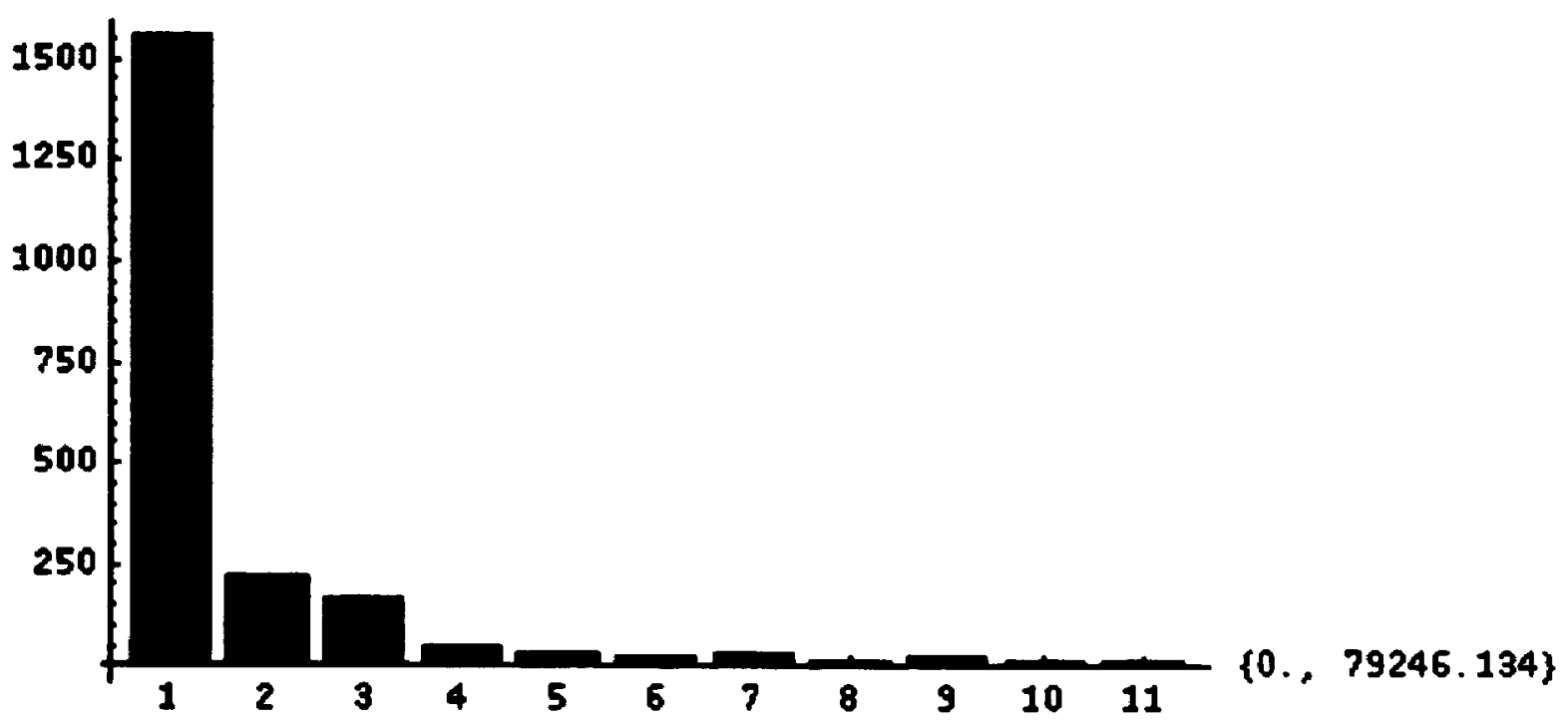

Figure 5.2 Distribution of file size loaded. Mean: 27516;

Standard Derivation: 322826; Confidence Interval: $\{14185,40936\}$ 


\subsection{The User Reading Time and Loading Time}

Most of time in a Mosaic session is spent on loading a file and reading the file loaded so that the loading time and the reading time are important, although they are not the system resource parameters. The distribution of both of them are closed to exponential distribution.

The loading time is mostly spent on the network, including connection, waiting and loading. The local system doesn't have much control on it. The loading times we measured are for the new fles loaded, not including those file in cache.

The reading time when the user stays/reads a loaded file is another important parameter because it would be the thinking time of the workload. It would determine the frequency of arrivals in performance model. In our Mosaic model, the reading time (thinking time) is the entire time when the user read, think, look around (move the mouse pointer and scroll bars) after a file is loaded. It even includes the idle time when the user is doing something else without working on the Mosaic session. Figure 5.3 and Figure 5.4 show the distribution of the reading time and loading time respectively. Similar to the cpu time distributions, the reading time distribution and the loading time distribution appears to be close to exponential. 


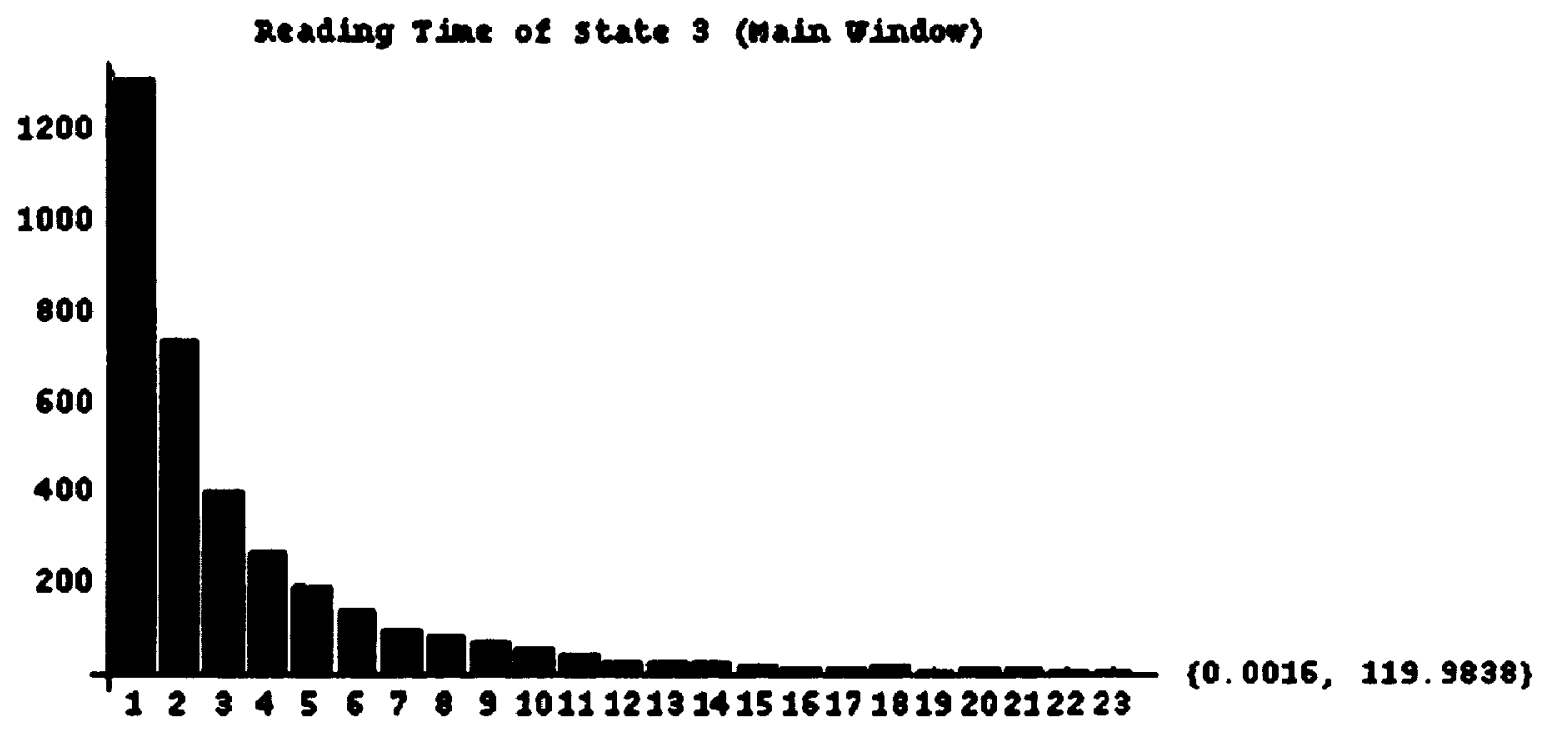

Figure 5.3. The Reading Time In State 3 (Main Window).

Mean:37.0125; Standard Derivation: 147.9771;

Confidence Interval on the Mean:\{32.3402,41.6902\}

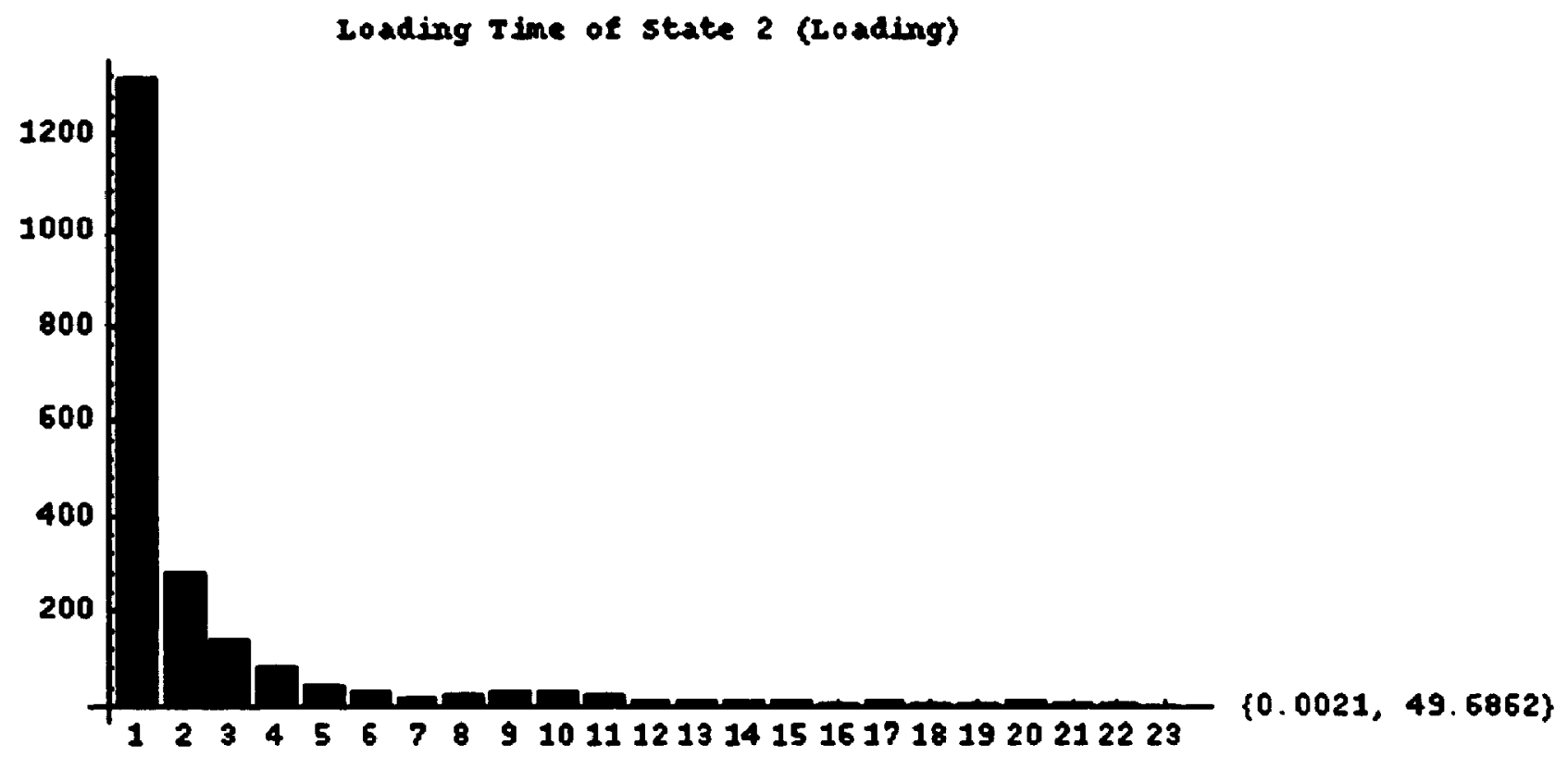

Figure 5.4. The Loading Time In State 2 (Loading)

Mean: 12.3732; Standard Derivation: 70.n166;

Confidence Interval on the Mean: $\{9.4721,15.2742\}$ 


\subsection{Other Parameters of Mosaic Model:}

As mentioned, the experiment measured as many system parameters as possible. Although some of them are not needed at the current time, they could be used in the further studies. Following are the parameter definitions in all of the states of Mosaic model:

Parameter 1: elapsed_time

The elapsed time in a state.

Parameter2: ru_utime

The total cpu time (in seconds) spent executing in user mode;

Parameter3: ru_stime

The total cpu time (in seconds) spent executing in system mode;

Parameter4: ru_maxrss

The maximum resident set size in pages ( $4 \mathrm{Kbytes} /$ page by default) when it is running;

Parameter5: ru_idrss

Amount of memory in use by the process while it is running;

Parameter6: ru_minftit

The number of page faults serviced which didn't require any physical I/O activity; Parameter7: ru_majflt

The number of page faults serviced which required physical I/O activity;

Parameter8: ru_nswap

The number of times the process was swapped out of main memory;

Parameter9: $u_{\text {_inblock }}$

The number of times file system had to perform input in servicing a read request; Parameter 10: ru_oublock

The number of times file system had to perform output in servicing a write request; Parameter 11: ru_msgsnd

The number of messages sent over sockets;

Parameter 12: ru_msgrcv

The number of messages received from sockets; 
Parameter13: ru_nsignals

The number of signals delivered;

Parameter 14: ru_nvesw

The number of times a context switch resulted due to the process voluntarily

giving up the processor before its time slice was completed;

Parameter 15: ru_nivcsw

The number of times a context switch resulted due to a higher priority process

becoming runnable or because the current process exceeded its time slice;

Parameter 16: Bytes_loaded

The file size loaded in "loading" state.

Parameter 17: lineCount

The number of lines of the current presented text in "main www window" state;

Parameter 18: anchorCount

The number of anchors the current text in "main www window" state.

Since there is a large quantity of data for all the states, we show them in Appendix C. With the same reason, we only give the mean, variance, standard derivation and confidence interval of all parameters measured, the distribution of the parameters are ignored. Here we just give the parameters of state 3 (main wincic:.) as a example in Table 5.3.

Some of parameters are not all meaningful for every state. For example, parameter 16 (Bytes loaded) is only meaningful for state 2 (loading), not for any other states. Parameter 17 (lineCount) and parameter 18 (anchorCount) are only for state 3 (main window), not for other states. 


\begin{tabular}{|c|c|c|c|}
\hline Paraneter & Mean & SD & $95 * C I$ \\
\hline $\begin{array}{l}1 \text { elapsed_tine } \\
2 \text { ru_utime } \\
3 \text { ru_stime } \\
4 \text { ru_maxrss }\end{array}$ & $\begin{array}{l}37.0152 \\
0.0999 \\
0.0338 \\
1357.518\end{array}$ & $\begin{array}{l}147.9771 \\
0.2997 \\
0.0705 \\
466.513\end{array}$ & $\begin{array}{l}\{32.3402,41.6902\} \\
\{0.0904,0.1094\} \\
\{0.0315,0.036\} \\
\{1342.7796,1372.2565\}\end{array}$ \\
\hline $\begin{array}{l}5 \text { ru_idrss } \\
6 \text { ru_minflt } \\
7 \text { ru_majflt } \\
8 \text { ru_nswap } \\
9 \text { u_inblock } \\
10 \text { ru_oublock } \\
11 \text { ru_msgsnd } \\
12 \text { ru_msgrcv } \\
13 \text { ru_nsignals } \\
14 \text { ru_nvesw } \\
15 \text { ru_nivcsw } \\
17 \text { lineCount } \\
18 \text { anchorCount }\end{array}$ & $\begin{array}{l}1.996710 \\
33.6092 \\
0.9525 \\
0 . \\
0.0099 \\
0.1838 \\
42.1161 \\
137.1288 \\
0 . \\
129.677 \\
33.0989 \\
88.8972 \\
31.336\end{array}$ & $\begin{array}{l}2.345110 \\
110.8486 \\
12.7434 \\
0 . \\
0.1609 \\
3.4605 \\
91.6131 \\
242.0182 \\
0 . \\
205.5729 \\
209.0512 \\
230.544 \\
82.4732\end{array}$ & $\begin{array}{l}\left\{1.922610,2.070810^{6}\right\} \\
\{30.1072,37.1112\} \\
\{0.5499,1.3551\} \\
\{0.0 .\} \\
\{0.0948,0.0149\} \\
\{0.0745,0.2932\} \\
\{39.2218,45.0104\} \\
\{129.4828,144.7748\} \\
\{0.0 .\} \\
\{123.1823,136.1716\} \\
\{26.4944,39.7035\} \\
\{81.6136,96.1807\} \\
\{28.7305,33.9416\}\end{array}$ \\
\hline
\end{tabular}

Table 5.3. Parameters of State 3 (Main Window) in Mosaic Model

\subsection{Response Cpu time, Response Rate and Utilization of Mosaic.}

More analysis from the Mosaic model in Figure 4.2 can be done. In this section, we will obtain the average cpu time and the response rates of response of the user's actions in this section. Then the utilization of Mosaic client workstation will be given.

\subsubsection{CPU time of the Response of user.}

As we know that Mosaic is a interactive application. Upon the user's input. the user actions start from "main_window" to different state, and complete when the action is completed and return to the "main_window" again, as shown in Figure 4.1. The completion of starting a user's action until the start of next action is the a response of the user action. In order to 
cbtain the cpu time, we define $y_{3 i}$ as the visit ratio of state 3 "main_window" to other states, as following:

$$
y_{3 i}=\frac{\text { Visits to state } i}{\text { Visits to state } 3}=\frac{\text { Occurrences of state } i}{\text { Occurrences of state } 3}
$$

The occurrences of states are shown in Table 4.2. and Table A.1, with the occurrences of state 3 as 4153 .

Let the cpu time of state $i$ per visit be $\mathrm{CPU}_{\mathrm{i}}$ which is shown in Table 5.1, then the average cpu time of a response of the user's action is $\mathrm{CPU}_{\Gamma}$ as following:

$$
\mathrm{CPU}_{\mathrm{r}}=\sum_{\mathrm{i}=1}^{16}\left(\mathrm{y}_{3 \mathrm{i}} \cdot \mathrm{CPU}_{\mathrm{i}}\right)
$$

Combine Table A.1 and Table 5.1, we show the visit ratio $\mathrm{y}_{3 \mathrm{i}}$ and the $\mathrm{cpu}$ time per response. $\mathrm{CPU}_{\mathrm{i}}$ /response (the last column), in Table 5.4 .

From the summary of last colum.a in Table 5.4, the average cpu time per response of the user's action is

$$
\mathrm{CPU}_{\mathrm{r}}=0.6863 \text { (seconds) }
$$


No. State Name Occurrences $\mathrm{y}_{3 \mathrm{i}} \quad \mathrm{CPU}_{\mathrm{i}} /$ visit $\quad \mathrm{CPU}_{\mathrm{i}} /$ response

$\begin{array}{llclll}1 & \text { Start } & 115 & 0 * & 2.1177 & 0 \\ 2 & \text { Loading } & 2423 & 0.5840 * * & 0.6115 & 0.3572 \\ 3 & \text { Main Window } & 4153 & 1.0000 & 0.1337 & 0.1337 \\ 4 & \text { Submit Format } & 108 & 0.0260 & 0.0051 & 0.0001 \\ 5 & \text { Open URL } & 147 & 0.0354 & 0.1744 & 0.0062 \\ 6 & \text { Reload } & 30 & 0.0072 & 0.0037 & 0.0000 \\ 7 & \text { Home } & 10 & 0.0024 & 0.0038 & 0.0000 \\ 8 & \text { Active Anchor } & 1859 & 0.4476 & 0.2198 & 0.0984 \\ 9 & \text { Back } & 1282 & 0.3087 & 0.1690 & 0.0522 \\ 10 & \text { Forward } & 90 & 0.0217 & 0.1722 & 0.0037 \\ 11 & \text { Key Press } & 91 & 0.0219 & 0.0808 & 0.0018 \\ 12 & \text { Hotlist } & 122 & 0.0294 & 0.2997 & 0.0088 \\ 13 & \text { Add Hotlist } & 48 & 0.0116 & 0.0275 & 0.0003 \\ 14 & \text { Printing } & 28 & 0.0067 & 0.2098 & 0.0014 \\ 15 & \text { Saving } & 223 & 0.0540 & 0.3353 & 0.0181 \\ 16 & \text { Exit } & 115 & 0.0277 & 0.1599 & 0.0044 \\ \text { Sum } & & & & & 0.6863 \text { (seconds) }\end{array}$

Table 5.4 Cpu time of response of the user's action.

Note in Table 5.4

* "Start" state is not invoked by the user from the "Main Window" so that it is not included in the user's responses.

** Although the "Loading" state is not visited by the "Main Window" directly, it is invoked by those states which are invoked by "Main Window" state (except the "Start" state), so that it should be included in the response.

\subsubsection{Response Rates of User's Actions and Utilization of Mosaic Client Workstation}

From the Mosaic model in Figure 4.1 and other measurement metrics, the response rate of user's actions and the utilization of Mosaic client on its workstation can be obtained. It is the speed of pushing buttons by the users. Let $X$ be the throughput of file loading requests to the 
local server, $a$ the ratio of the throughput of requesting remote servers to $X$,

$$
a=\frac{\text { Throughput of requesting remote servers }}{\text { Throughput of requesting local server }}
$$

and $\mathbf{r}$ the ratio of response rate to the file loading rate:

$$
r=\frac{\text { response rate }}{\text { file loading rate }}=\frac{\text { occurrences of actions }}{\text { occurrences of loading }}=\frac{4153}{2423}=1.714
$$

Then, the response rate contributing to the local file lcading requests is

$$
\text { R_rate_l }=(r \bullet X)
$$

The response rate contributing to remote file loading is

$$
\text { R_rate_r }=(a) \cdot(r \cdot X)
$$

Clearly, the Mosaic client utilization of the workstation is:

$$
\mathrm{Uc}=\left(\mathrm{CPU}_{\mathrm{r}}\right) \cdot(1+\mathrm{a}) \cdot(\mathrm{r} \cdot \mathrm{X})
$$

there, CPUr is the cpu time per response, as described in section 5.5.1.

Usually, the local users consider the local server as a home page or a reference page to other server so that we assume all of the local user use the local server as their home page. And also we assume that the time in which the users are reading on the same document should be less than 1 hour since most of users wouldn't spend a very long time to read a document. If this reading time is more than 1 hour, we assime that the client is idle, and that the user starts a new sessien of WWW client. With these conditions, we obtained the average throughput of loading local files is $\mathrm{X}=0.0018 / \mathrm{second}$. With the same assumption, the ratio of the throughput of requesting remote servers to $X$ is $a=15$. Then, we have: 
R_rate_l: $\quad 0.0031 /$ second, response rate contributed to local file loading requests.

R_rate_r: $\quad 0.0463 /$ second, total response rate to an average user's action.

Uc: 0.0338 , workstation utilization by a user.

\subsection{Summary}

One of the major work of workload characterization is to measure and obtain the system resource demands. With the model of User Behavior Graph of Mosaic, these parameters are associated with different states. They are measured in every state of the model. The parameters are given in mean, standard derivation and confidence intervals. The cpu times are the most important parameters of the states. Th.ey have nearly exponential distributions. "Loading file" is the nost cpu consuming state in Mosaic. The reading time of a document and the delay of loading a new file are the other important parameters for the purpose of workload characterization and user behavior. Their distributions are all close to exponential. More parameters with different states are mostly shown in Appendix B and Appendix C. And most of their distributions appear to be roughly exponential. 


\section{Chapter 6}

\section{The Performance Model of WWW System}

The whole WWW system, including clients and servers, has a significant impact on system performance. For the performance of a local system which runs a WWW server(s), the server side may be more important. A busy server could receive thousands of requests (here and later, we refer a "request" to be for loading one file) a day. It is necessary to analyze the performance of the WWW system with both client and server together. In this chapter, we will build an analytic performance model of a WWW system, including client and server. The parameter data of client and server are derived from the measurements reported in chapter 5 , plus some additional measurements as described in Chapter 3. Since it is impossible to measure the servers outside of our local system, the WWW server, which is running in The System and Computer Engineering Department at Carleton University is chosen as the target of measurement for the WWW system model.

\subsection{The Model of WWW System}

We describe the WWW system model as a queuing network model, as shown in Figure 6.1.

A WWW client can access different servers. And a WWW server also can receive requests either from the local system or from the remote site of the internet. The remote requests come to the local server, get access and leave. The local WWW requests to the server are inside the local system. For the model in Figure 6.1, the remote request is the open class, and the local request is the closed class. Therefore, the model is a mixed classes model with open class for remote requests and closed class for local reques $:$ 


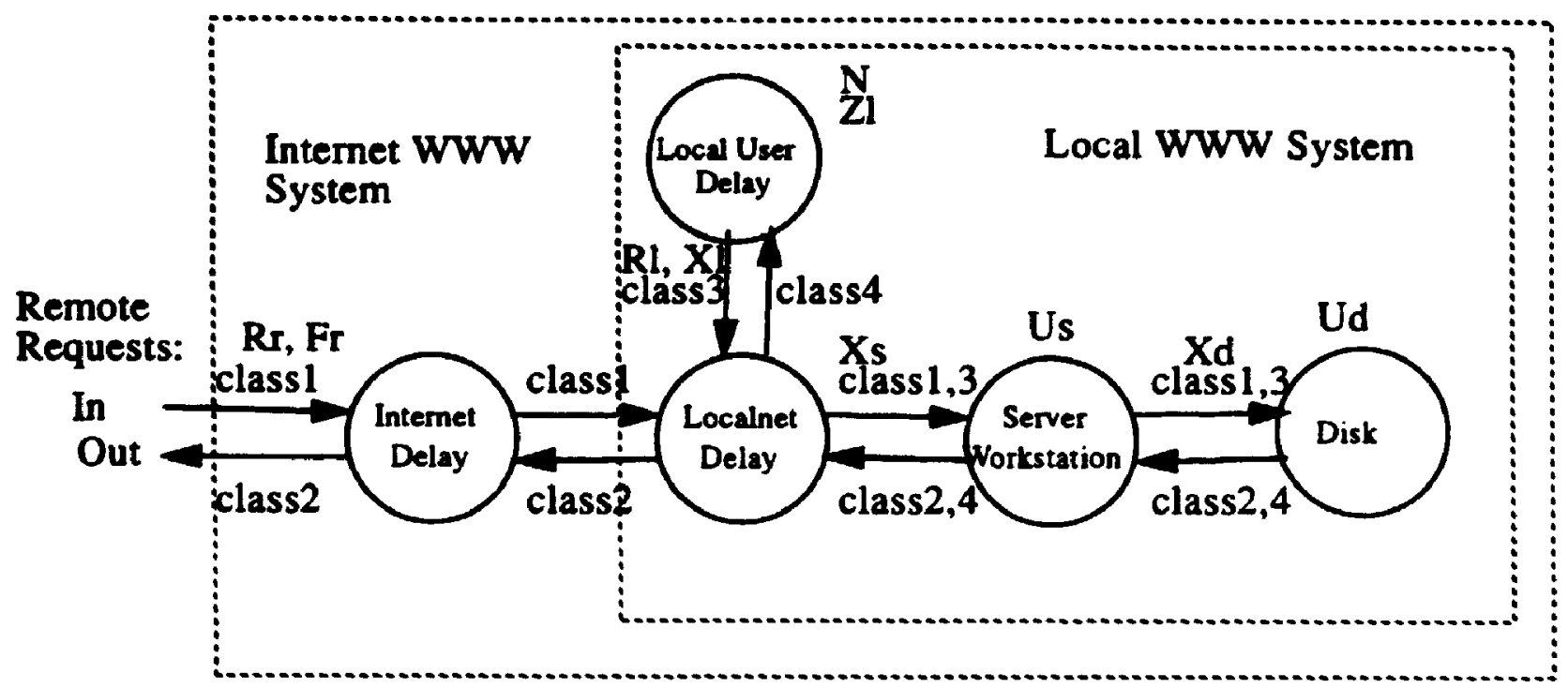

Fr: The arrival rate of remote requests (1/s). One request is for loading one file.

$\mathrm{N}$ : The Number of local users

$\mathrm{Rr}$ : The response time of remote requests (s).

$\mathrm{Rl}$ : The response time of local requests (s).

$X I$ : The throughput of the WWW requests of local users $(1 / \mathrm{s})$.

Xs: The throughput of total WWW requests, including remote and local requests (1/s).

$\mathrm{Xd}$ : The same as Xs, but for the Disk

Us: The utilization of server.

Ud: The utilization of disk.

Zl: The Surrogate Delay of local users.

Figure 6.1 Performance Model of WWW System

In the model of Figure 6.1, it is clear that the Server and Disk are queuing service centers. The Local User Delay is the local workstations on which the WWW clients work. Theses workstations are represented as an infinite service center (since there is one workstation per client, there is no queuing). We call it the Local User Delay center, a surrogate delay center. Its delay time is the time between two access request to the local server, which is measured from the measurement of Mosaic. The network delay varies widely and is not really altered by the load due to one subsystem, so we can assume the network center are delay centers. They have random delays with their mean values, which may be class dependent. So we assume the Internet and Localnet are delay centers. For the server, there are other process which are requesting the server when the WWW clients request it. In order to simplify the 
model, we reduce the speed of the server without modelling the additiona? classes by using the load concealment technique [24], p145-146. Let $D_{w, k}$ is the demand of some WWW requests on service center $k$, and let Uo,k is the utilization of other classes on service center $k$. Then the transformed demand of WWW request with reduced service speed on service center $k$ is

$$
D^{\prime} w, k=\frac{D w, k}{1-U o, k}
$$

This formula modify the demand in order to simplify the model. It will be used in the following discussion.

The parameters needed in the model are mostly from the measurement. Since the measurement data are limited, we need some assumptions to solve this model. Following are the assumptions for the model:

1. The accesses from a client to other servers are not included in the model since we analyze with only one WWW server, our surrogate server. That is, only the access to this specific server are considered. All of the time spent on loading file from other server sites is considered as part of the local user surrogate delay $\mathrm{Zl}$ of users, since they are inactive from the point of view of the local server.

2. The differences of the files size accessed through the server are ignored. We just take the average file size access among all the files the server provides. This mean file size is needed to determine the demand on server and disk.

3. Remote accesses to the server are modelled by a Poisson traffic source. This is reasonable if there are large number of independent users of the remote requests to the local system.

With the assumptions above, the model is a product form queueing network model. We can 
use product form method, Mean Value Analysis method, for example, to solve this model.

As explained before, this model is a mixed class model. One is open class for the remote requests. The other one is for local user requests. In order to simplify the model, we break each of the classes into 2 subclasses which are really shown in the model of Figure 6.1. For the remote requests, the two subclasses are:

class 1: It is the arrival of the remote requests. It goes through the Internet Delay, Localnet Delay, Server and Disk. And then it changes to class2 after leaving the Disk.

class2: It is the returning of the remote requests. It is changed from class 1 after visiting Disk, and then goes to Server, Localnet Delay, Internet Delay, and then leaves the system.

Therefore, with the 2 classes, one remote request visits Internet, Localnet and Server twice, and visits Disk only once.

For the local user requests, the two subclasses are:

class3: the arrival of the local user requests. It comes from the Client Workstation, and goes to Localnet Delay, Server and Disk. After leaving Disk, it changed to class4.

class4: the returning of the local user requests. It is changed from the class 3 after leaving the Disk, and then goes to Server, Localnet Delay and returns to Client Workstation. On arriving to Client workstation, it becomes class 3 again.

So for one local user request, it visits Localnet and Server twice, and visits Client Delay and Disk once.

\subsection{Parameters of the WWW System Model}

One of the major work in our measurement is to obtain the parameters which are needed in 
WWW performance model (Figure 6.1). These parameters are obtained in different ways, mostly from measurement. Some of them are from internet statistical data

The measurement design for the server is give in Chapter 3. We use the system accounting facilities to get some statistical data of the server side. The server has been monitored for about 9 days. And the following useful information is recorded:

1 The real time from the start to the end of running the server: 12741 minutes

2 The total cpu time of the WWW server: 4.46 minutes

3 The total cpu time of other processes: 321.10 minutes.

4 The number of disk I/O operations of the server process: 19399

In addition, the server itself maintain some logging files. One of them is the request logging file which records every request to the server. The data includes the address of the WWW client and the time when requests arrive to the server. During the measurement time, there are 5214 requests to the server. From the server logging files and the accounting logging file together, we obtained the average cpu time and average number of disk $\mathrm{L} / \mathrm{O}$ for one request, which are $51 \mathrm{~ms}$ and 3.72 respectively. This disk $\mathrm{I} / \mathrm{O}$ is not for caching since the disk $\mathrm{I} / \mathrm{O}$ are measured from the real system. As we mentioned in 6.1, there are some other process running on the same cpu. The transformed cpu time by reducing the cpu speed to account for the utilization of the other processes, which is about $2 \%$, is 52 ms.

Since we do not have the service time of the disk, the statistics of service time of some disks are obtained by using "iostat" system command. And we use the average disk service time in our model. which is about $46.44 \mathrm{~ms}$. This seems reasonable according to some document [30] [37], which state the disk service time is $10-60 \mathrm{~ms}$. Then the demand of one request on the disk would be $3.72 \times 46.44=173 \mathrm{~ms}$.

The internet delay could vary over different paths in the net, and we don't have the internet delay time from measurement. However, we can get some statistical data of some internet backbones. Here we use the statistical data of traffic in NSFNET (National Science Founda- 
tion Network) backbones, which are reported by Merit Network Information Center Services. From these data, we can get the average one way delay time among the nodes of NSFNET in April of 1995, which is $24 \mathrm{~ms}$ per packet.

In order to get the total delay time of one file for internet delay and local net delay, we need to know size of the files and the size of a internet packet. An internet IP packet is allowed to be 46 - 65536 bytes, but normally it is limited to 576 bytes. The reasonable size of an IP packet is 512 bytes [38 p.36] [39].

During the measurement time, the total bytes requested is 39867152 , and the total requests to all the files are $\mathbf{5 2 1 4}$ as mentioned above. Therefore, the average file size is $\mathbf{3 9 8 6 7 1 5 2 / 1}$ $5214=7646$ bytes for one request. And the average internet one way delay time for one request is $7646 / 512 * 21=358 \mathrm{~ms}$, assuming that packets are sent one at a time, and that the source of delay is at the sending station. The results of the performance model do not depend strongly on this number being accurate.

For the local net delay, we found that the average time of sending a 512 bytes message to the server workstation is about $3 \mathrm{~ms}$, by using system command "ping". Again with 7646 bytes of average file size, the local net delay is $44 \mathrm{~ms}$ ( $22 \mathrm{~ms}$ for one way).

The local user delay is the user behavior. The actual mean reading/thinking time in the "main window" state (refer to Figure 4.1) is about 37 seconds, as reported in Table 5.3. However, the local user surrogate delay is the time between tow request to the local server instead of the real reading/thinking time, as mentioned in 6.1. The time spent on requests to other servers and on other actiones of local users is considered as part of the local user surrogate delay. As explained in last chapter, the local users usually consider the local server as a home page or a reference page to other server so that we assume all of the local user use the local server as their home page. And also we assume that the time in which the users are reading on the same document should be less than 1 hour since most of user; wouldn't spend long time to read a document. If this reading time is more than 1 hour, we assume that the client is idle, and that the user starts a new session of WWW client. With these conditions, we obtained the 
average local user surrogate delay time from the Mosaic logging data, which is $563.757 \mathrm{sec}-$ onds. The long time local user delay may be because the local users are not interested in the local server too much.

Summarily, the parameters of the WWW system model are shown in Table 6.1, which are described as the demands of the service centers for one WWW request.

Node Service Time Visits Demand

$\begin{array}{llll}\text { Server: } & 26 \mathrm{~ms} & 2 & \mathrm{Ds}=52 \mathrm{~ms} \\ \text { Disk: } & 173 \mathrm{~ms} & 1 & \mathrm{Dd}=173 \mathrm{~ms} \\ \text { Localnet Delay: } & 22 \mathrm{~ms} & 2 & \mathrm{Dl}=44 \mathrm{~ms} \\ \text { Internet Delay: } & 169 \mathrm{~ms} & 2 & \mathrm{Di}=358 \mathrm{~ms} \\ \text { Local User Delay: } & 564 \text { seconds } & 1 & \mathrm{Z}=564 \text { seconds }\end{array}$

Table 6.1. Parameters of WWW System Model

\subsection{Analysis of the Performance Model}

The Qnap2 package was used to solve the model. Qnap2 is an system for describing, handling and solving queueing network models. We will analyze the model to find the impact of both of the remote requests and local requests on the system performance. The performance metrics we will analyze include response time, throughput, and utilization.

\subsubsection{Performance Effect of Remote Request Fr with One Local User}

First, we will study the impact of the remote arrival rate of requests, $\mathrm{Fr}$, by keeping the number of local users constant as 1 . Apparently, the bottleneck of the model is the disk which has the maximum service demand. Let Dmax is the largest service demands among the queue service center. The saturation of the arrival rate of the remote requests $\mathrm{Fr}^{*}$ is following, 
[21]p.73.

$$
\mathrm{Fr}^{*}=\frac{1}{\mathrm{Dmax}}=\frac{1}{0.173}=5.78 / \text { second }
$$

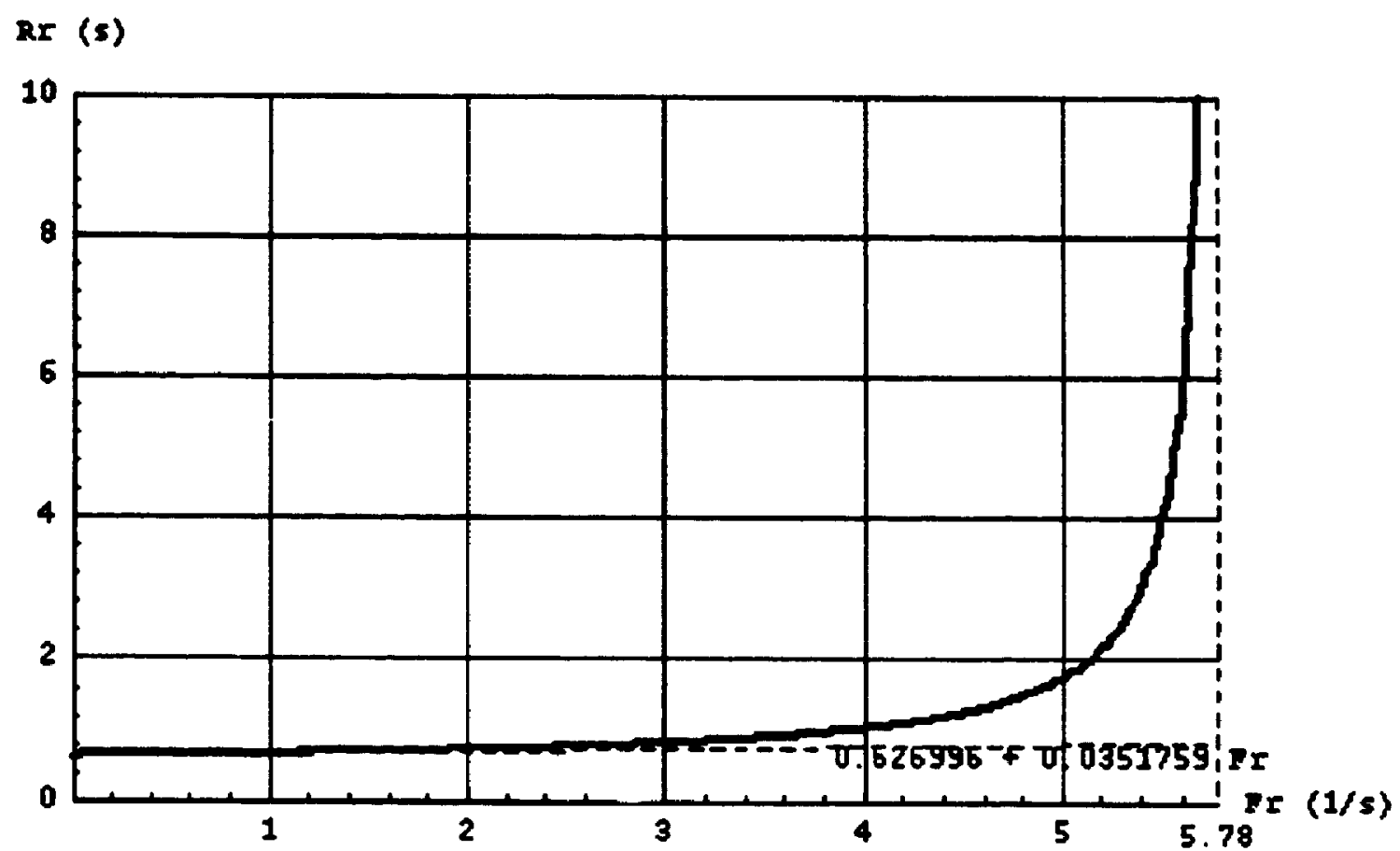

Figure 6.2.1. Response time of remote request $\mathrm{Xr}$ vs remote arrival rate $\mathrm{Fr}$, with 1 local user

Figure 6.2.1, 6.2.2, 6.2.3 and 6.2.4 show performance of the model with 1 local WWW user vs the remote arrival rate. They are the response time of the remote requests $\mathrm{Rr}$, the throughput of total requests in the local system Xs, the throughput of the local requests XI. the utilization of the server cpu Us and the utilization of the disk Ud. The response time of the local user is not shown here because it is almost the same as the response time remote requests. The only difference of the response time between the remote requests and local requests is that the local requests do not have internet delay which is $\mathrm{Di}=358 \mathrm{~ms}$. Then, the relationship of the response time of remote requests, $R r$, and the response time of the local users, $R l$, is:

$$
\mathrm{Rr}=\mathrm{RI}+358 \mathrm{~ms}
$$


$X s(1 / s)$

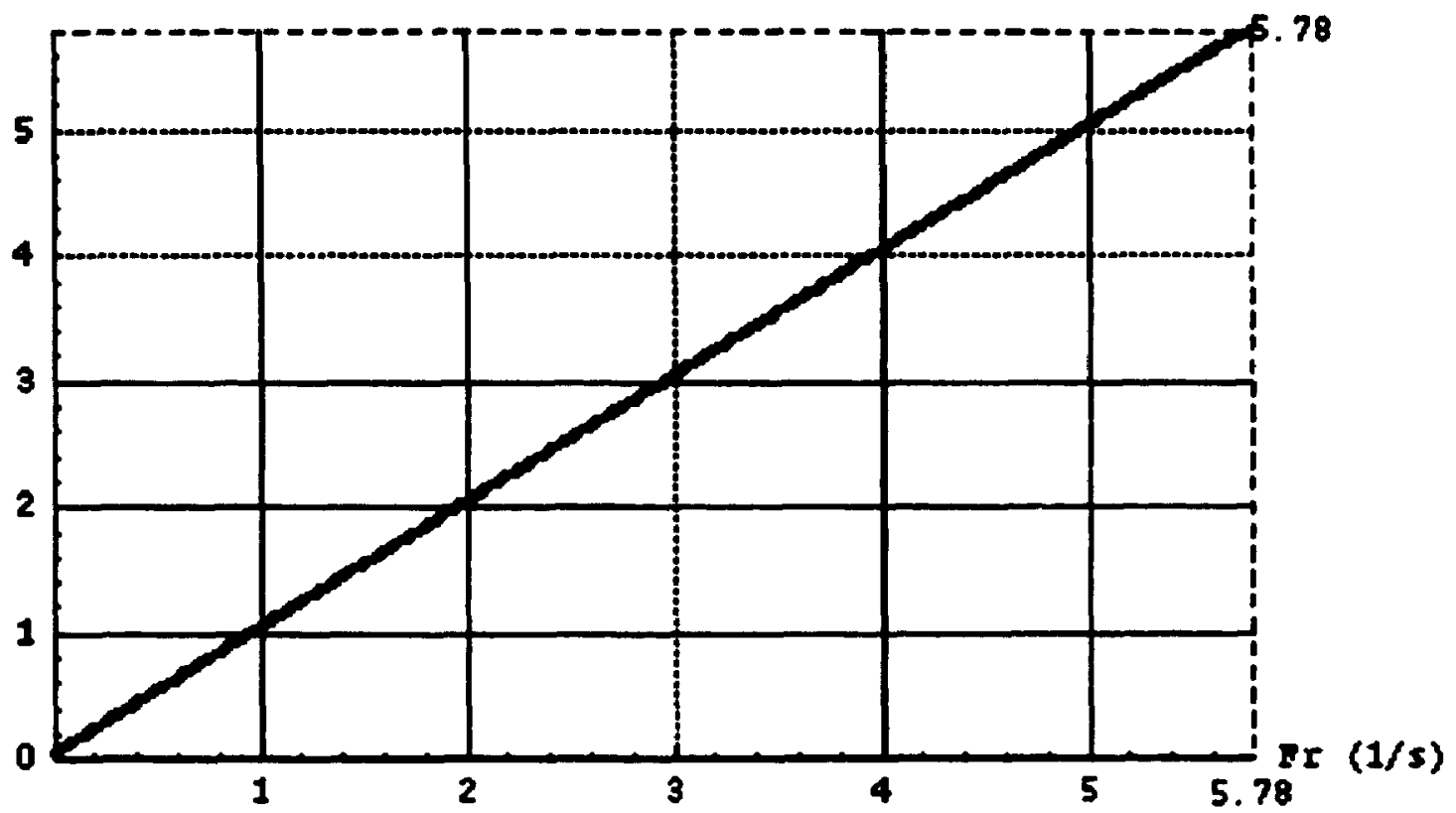

Figure 6.2.2. Total request throughput $\mathrm{Xs}$ vs remote arrival rate $\mathrm{Fr}$, with 1 local user.

$x 1(1 / s)$

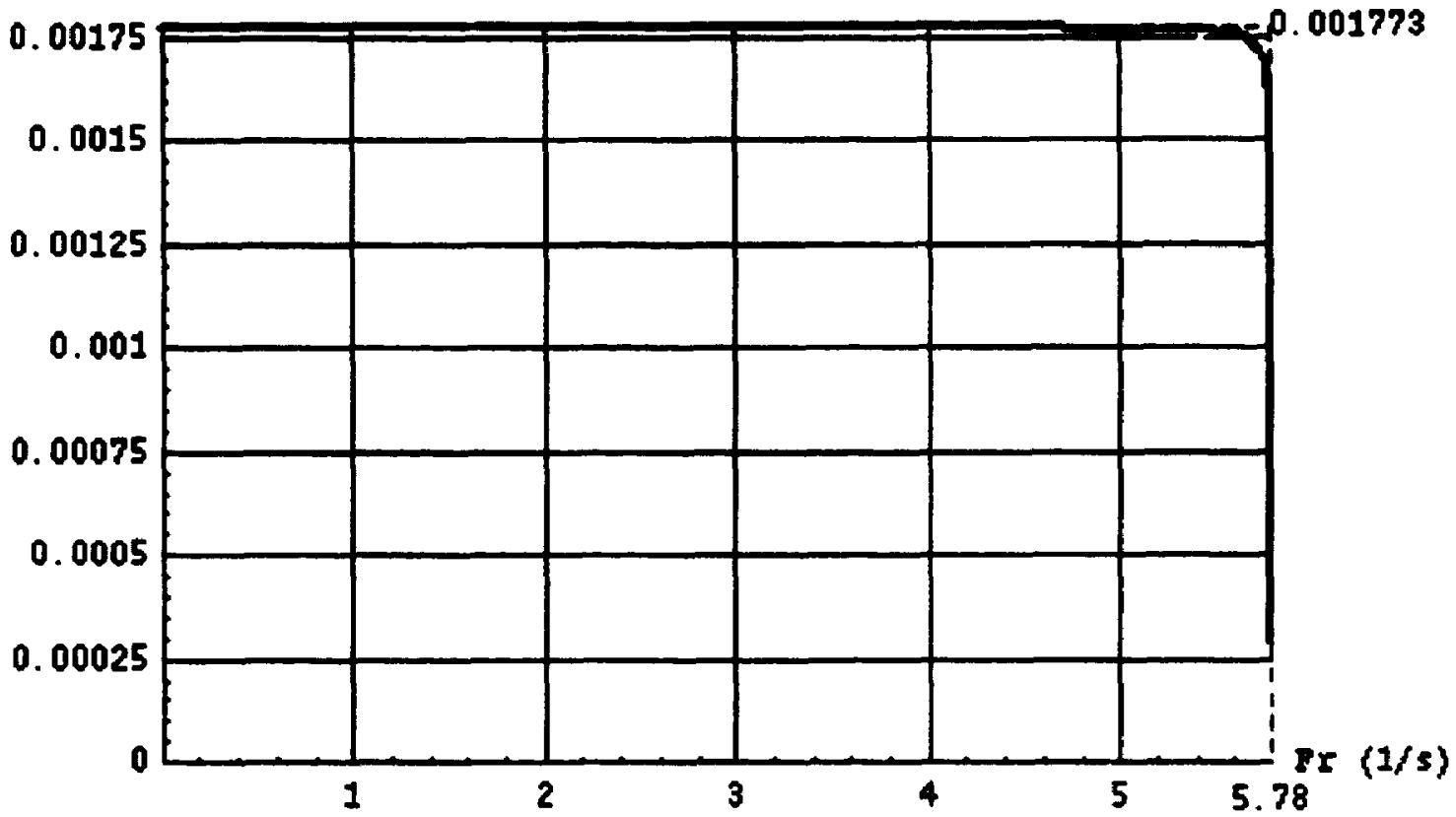

Figure 6.2.3. Local request throughput $\mathrm{XI}$ vs remote arrival rate $\mathrm{Fr}$, with 1 local user 


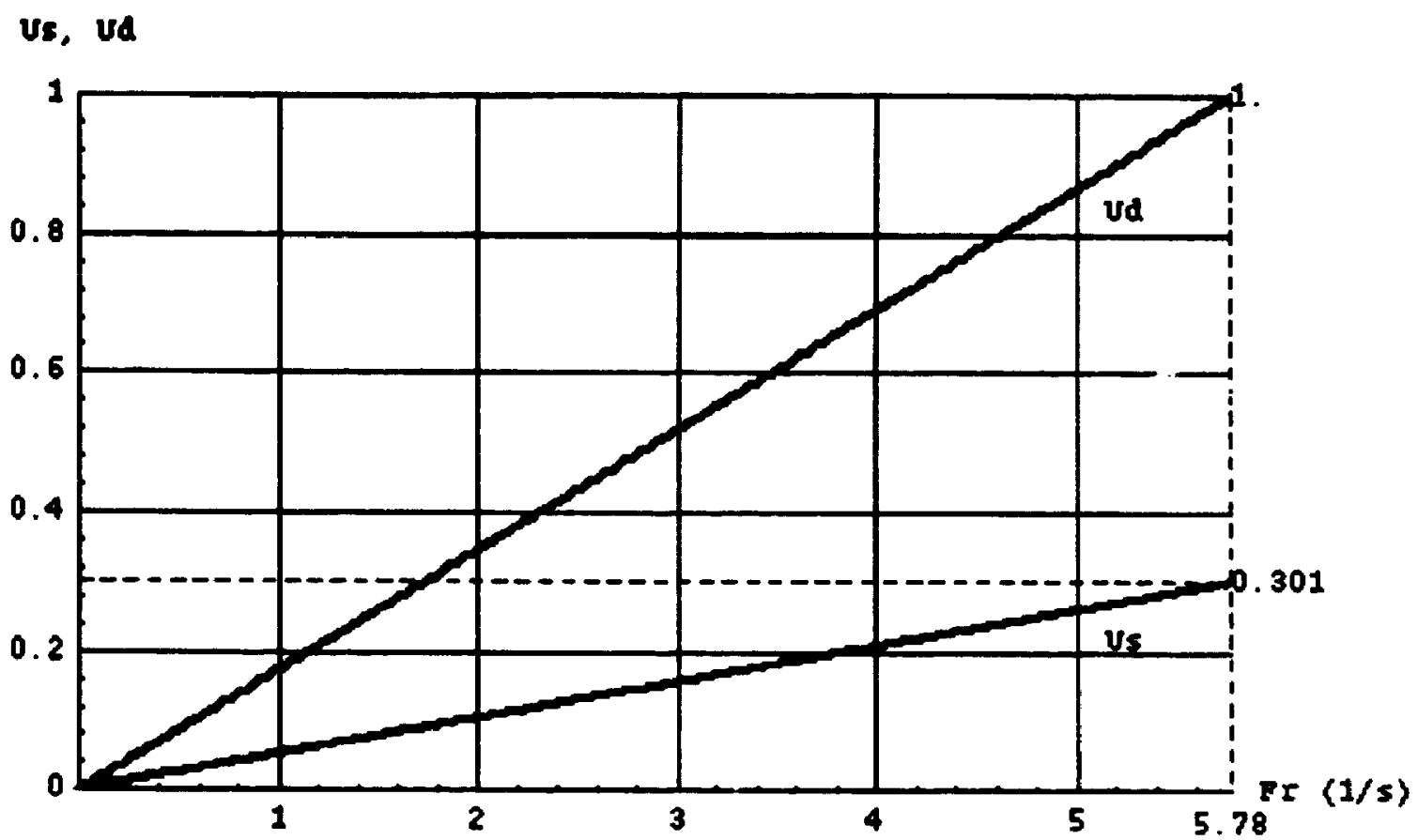

Figure 6.2.4. Utilizations of the server Us and disk Ud, vs remote arrival rate $\mathrm{Fr}$, with 1 local user

In the figures, we refer to the throughput as the throughput of WWW requests instead of the throughput of jobs. The response time is represented in seconds. And the remote arrival rate is represented in the number of requests per second.

This situation in Figure 6.2.1 to Figure 6.2.4 is the analysis of the model for remote request varying from 0 to the saturation $\mathrm{Fr}^{*}$. Since the number of the local users is very small, the system almost work with remote requests.

From the performance, we can see when the remote arrival rate, Fr, is no lose to its saturation, 5.78, the response time only varies slightly with the increment of the remote arrival rate. although the utilizations of server and disk keep increasing quickly. But when the remote arrival rate is reaching its saturation, the utilization of the disk, Ud, and throughput of total requests in the local system, Xs, are also reaching their saturations, 1 and 5.78. respectively, and the responses time of remote requests, $R r$, and the response time of local user requests, Rl, are increasing dramatically. From Figure 6.2.1, we assume that the maximum useful 
response time of remote requests is $\mathbf{2}$ seconds since the response time will increasing dramatically when the response time is more than 2 seconds. At this point, the remote arrival rate is about $5.13 /$ second. Therefore, the system can only work with remote arrival rate up to stout $5.13 /$ second with one local user. Beyond this rate. remote requests should perhaps be discarded.

The throughput of local user requests, $\mathrm{XI}$, in the system is decreasing with the increase of the remote arrival rate. This because there are more jobs in the system when the remote arrival rate is bigger, and so that the waiting time on server or disk becomes bigger. But the decrement of the $\mathrm{Xl}$ is not significant when the system is working in light load. However, when the remote arrival rate is big or close to its saturation, $\mathrm{XI}$ decreases dramatically. This is because the throughput of the system is the summary of the remote arrival rate and local user throughput. And it has to follow the following rule:

$$
(\mathrm{Fr}+\mathrm{X}]) \leq \mathrm{Fr}^{*}
$$

When remote arrival rate $\mathrm{Fr}$ is reaching the saturation $\mathrm{F}^{*}$, the local user throughput $\mathrm{Xl}$ is becoming to 0 . But as we mentionei that the system can only work properly when the remote arrival rate Fr is less than 5.13/second, the local user throughput almost keeps constant in this range so that there is no effect on the local request throughput from the remote arrival time. This is because the local users delay $\mathrm{Zl}$ is very big compare the total demand $\mathrm{D}$. And we have $\mathrm{D} / \mathrm{Zl}=629 / 563757=0.1 \%$ so that the local users are reading or doing other working instead of request to the local server in the most time. And there is not much effect even just before the utilization of the disk reach to 1 .

The total request $L^{\prime}$ roughput in the system, $X s$, is linear and so are the utilizations of server and disk because of the small number of iocal users. This means small number of local users cioesn't affect the remote request significantly.

The utilization of the disk and server reach $\subset 89$ and 0.27 respectively when the arrival rate is 
5.13. These are the $89 \%$ of their maximum useful utilizations.

\subsubsection{Performance Effect of Remote Request Fr with 100 Local Users}

There is only one local user in the performance analysis in section 6.j.1. Normally, there should be more local users. In this section, we will analyze the performance again with 100 local users, each with a personal workstation.

In order to reduce the number of figures in the text, the figures of the performance are shown in Figure D.4.1 to D.4.4 in Appendix D. Again, they are the response time $r f$ the remote requests $\mathrm{Rr}$, the throughput of total requests in the local system $\mathrm{Xs}$, the throughput of the local requests XI, the utilization of the disk Us and the utili: dion of the Ud. Here we just show the response time of remote request in Figure 6.3.1.

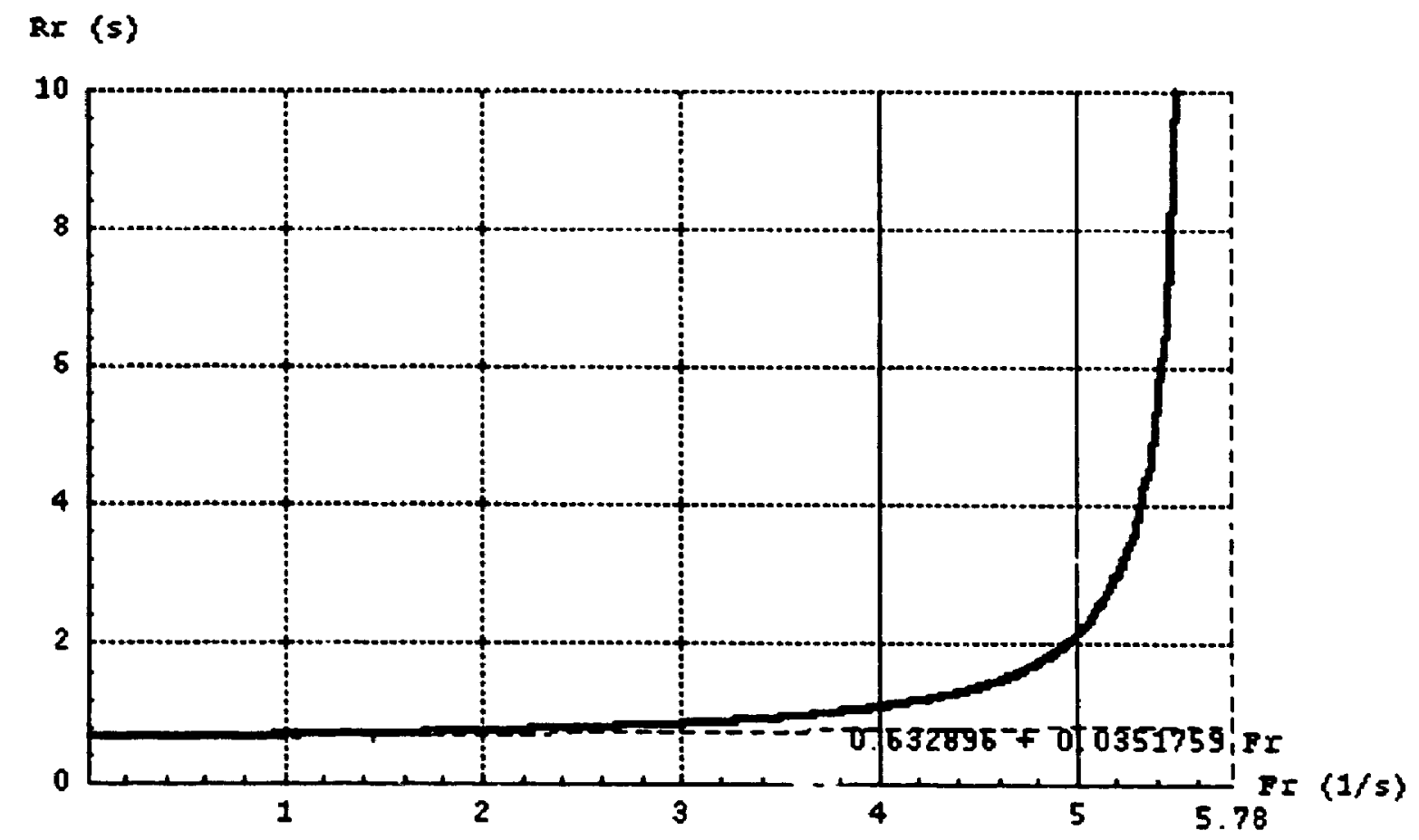

Tigure 6.3.1. Response time of remote request $\mathrm{Rr}$ vs remote arrival rate $\mathrm{Fr}$. with 100 local users

In this situation, both remote and local requests are requesting the local server. If the maxi- 
mum useful response time of remote requests is still 2 seconds, as assumed in section 6.3.1, then the maximum useful arrival rate of remote requests is $4.95 /$ second. Therefore, more than 4.95/second of remote requests should be denied if we want to keep up to 100 local users :0 request the local server. At this point, the remote arrival rate is $4.95 / 5.78=86 \%$ of its saturation.

Compare Figure 6.3.1 and Figure 6.2.1, we can see that the response time of the remote request increase when the number of local users changes from 1 to 100 . And the increasing is also faster with the bigger remote arrival rate, as show in Figure 6.4.1 which give the absolute difference of Figure 6.3.1 and 6.2.1.

$\operatorname{Rr}(100)-\operatorname{Rr}(1)(5)$

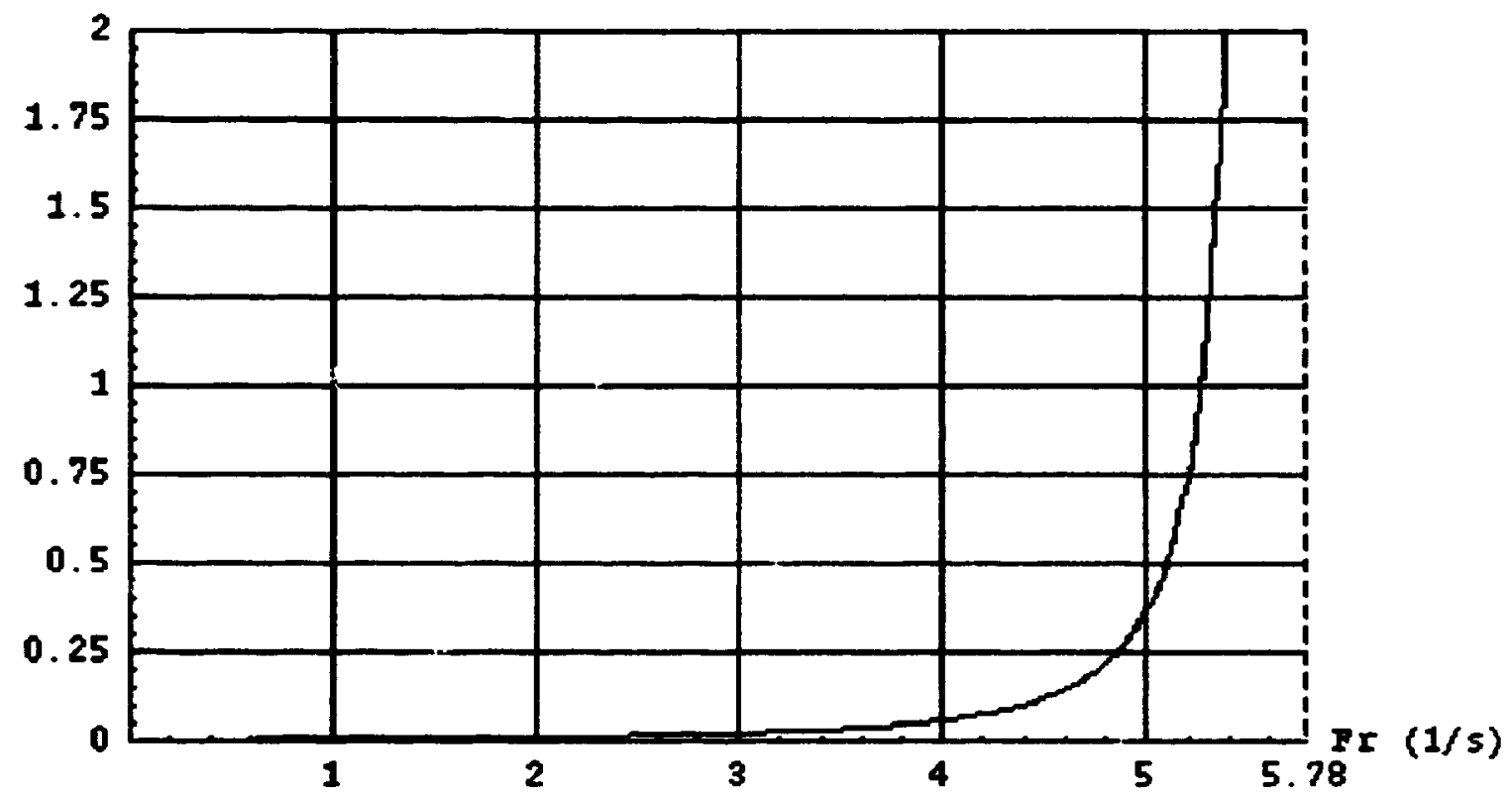

Figure 6.4.1. The difference of remote response time of 1 local user from 100 local users vs the remote arrival rate $\mathrm{Fr}$

When the system is working in light load (e.g. remote arrival rate is less than 4.95), the increasing of remote response time is small, as shown in Figure 6.4.1. But when it is working in the heavy load with remote arrival rate bigger than 4.95 , the increase beccmes dramatic. For example, the relative difference at remote arrival rate 1 is $1 \%$, but it becomes $137 \%$ when 
remote arrival rate is 5.5. This implies that the increasing number of local users have significant interfere on the remote requests only when the system is working in heavy load.

And also, when the remote arrival rate is less than 4.95, the response times between 1 local users and 100 local users are small as shown in Figure 6.4.1. Since most WwW clients like Mosaic and Netscape are window based programs, it is reasonable to consider that every workstation only has one WWW user. In the System and Computer Engineering Department at Carleton University where I am working on this thesis project, there are about 100 workstations which are able to run window based WWW clients, so that the system can only have at most 100 local WWW users at the same time. Therefore, the local WWW users don't have much impact on the WWW system, when the remote arrival rate is less than $4.95 / \mathrm{sec}$.

Another fact we can see, from these figures, is that the model is still stable before the arrival rate reaches its saturation, 5.78, even though the utilization of disk and throughput of the system are keeping in their saturations due to the local user requests (refer to Figure D.4.1 to D.4.4 in Appendix 4). But the response time would be too big. For example, the utilization of disk becomes to 1 when the remote arrival rate is 5.64 , the remote response time is $151 \mathrm{sec}$ onds, as shown in Figure D.4.1 and D.4.4. However, with more local users, the system would only work properly with less remote arrival rate. Otherwise, the response time would be to big to request the server. Therefore, less remote requests would be allowed to request the local server. This situation could be seen more clearly from Figure D.1.1 to D.1.4, in Appendix $D$, which show the performance of the model with 500 local users.

\subsubsection{Local User Performance with No Remote Requests}

In section 6.3.1 and 6.3.2, the performance analysis of the model are for varying rates of the remote requests. In this section, we will analyze performance of the model with varying of the local user class, keeping the remote arrival rate as $0.001 /$ second which is very small, as shown in Figure 6.5.1 to 6.5.4. 


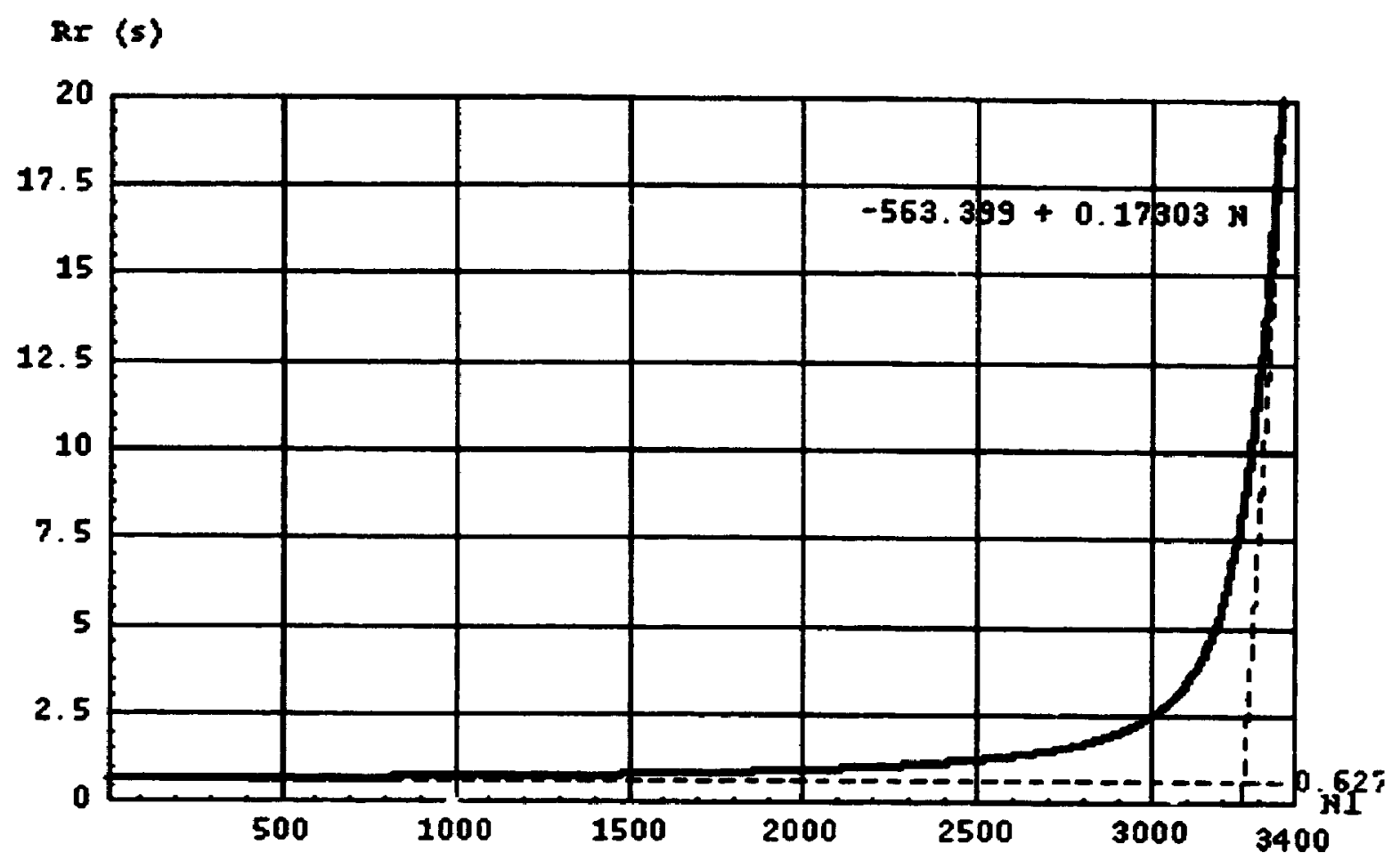

Figure 6.5.1. Response time of remote requests Rr vs the number of local users $\mathrm{Nl}$, with remote arrival rate 0.001

$x s(1 / s)$

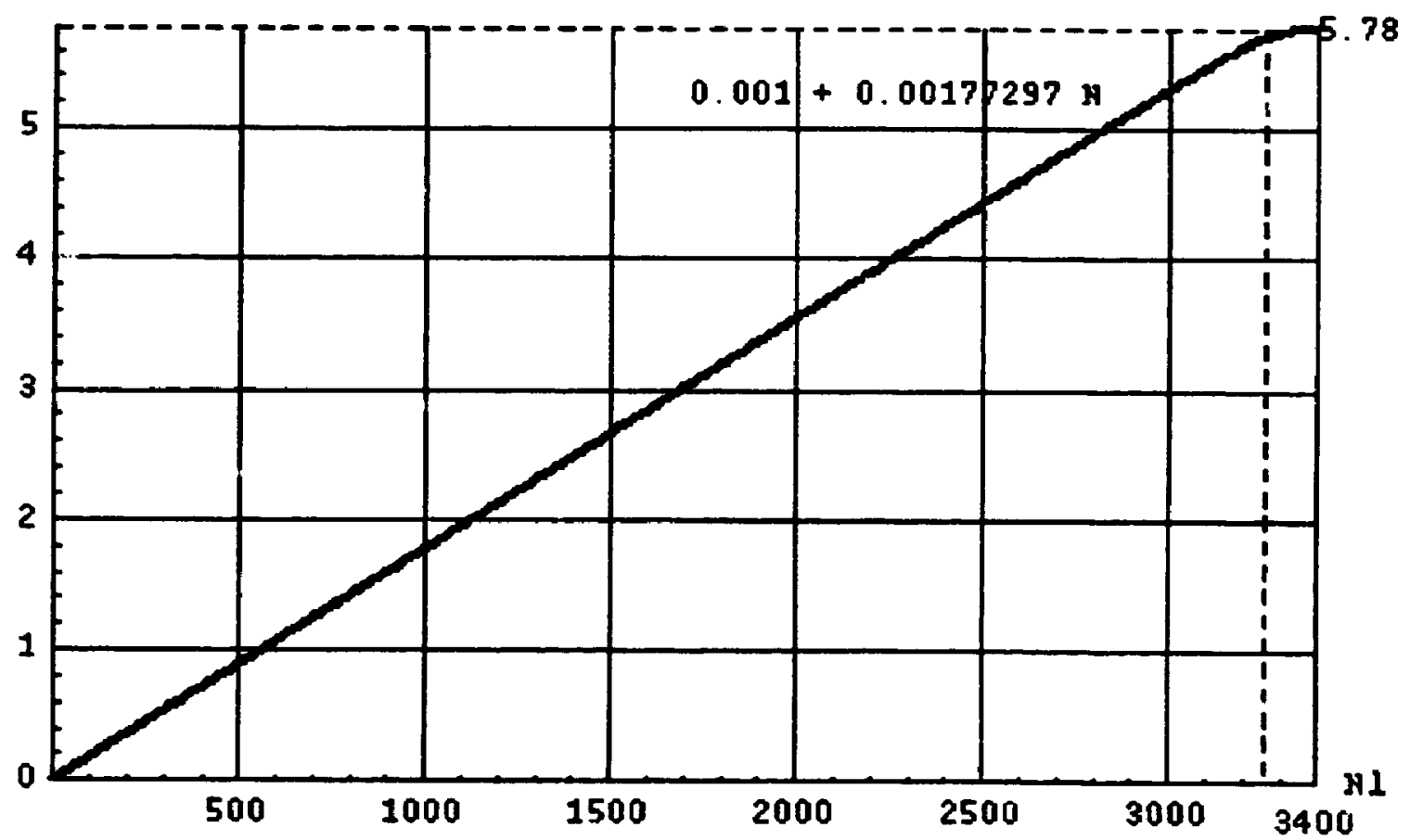

Figure 6.5.2. Total request throughput $\mathrm{Xs}$ vs the number of local users $\mathrm{Nl}$, with remote arrival rate 0.001 
$x 1(1 / 5)$

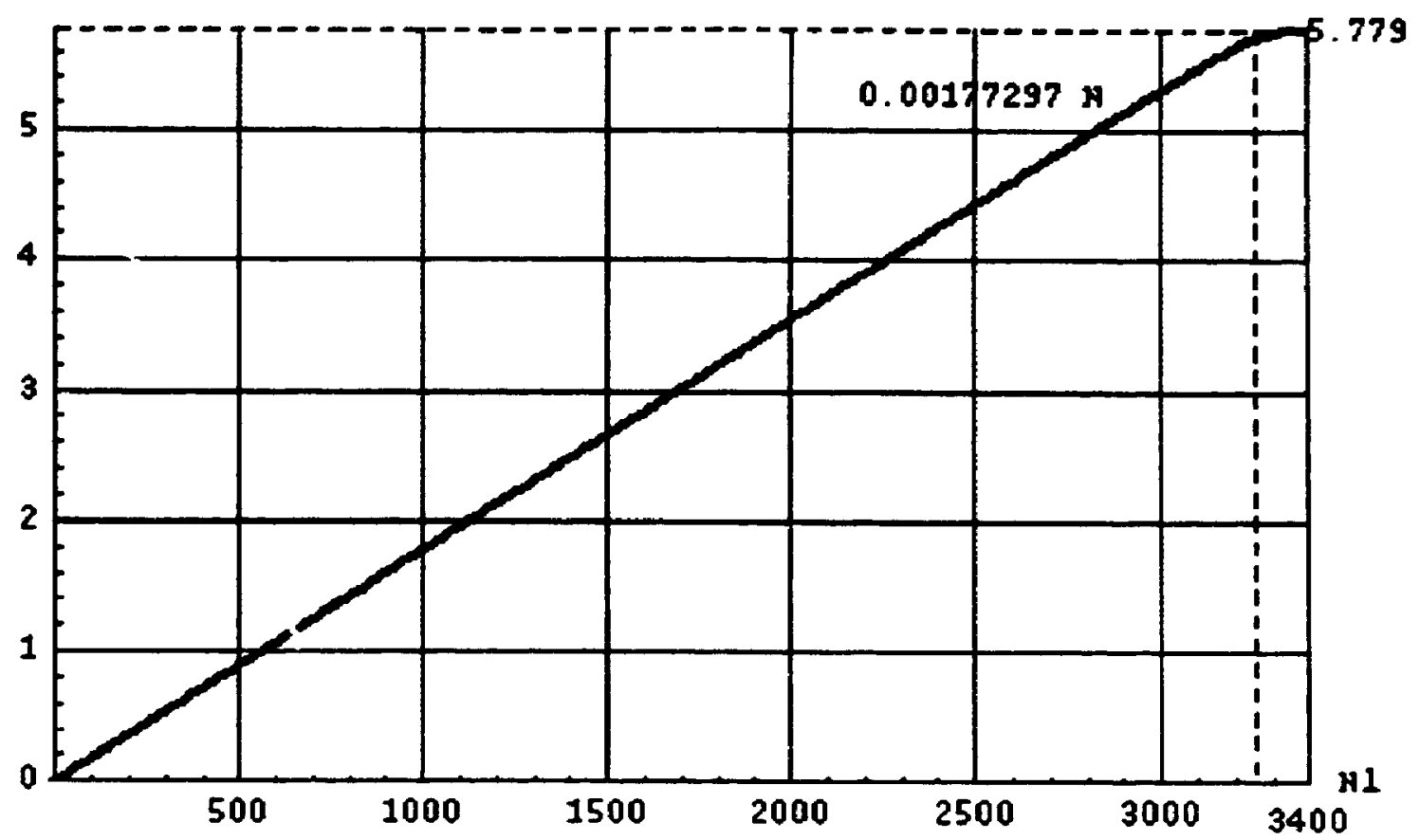

Figure 6.5.3. Loca! request throughput $\mathrm{Xl}$ vs the number of local users $\mathrm{Nl}$, with remote arrival rate 0.001

Us, vd

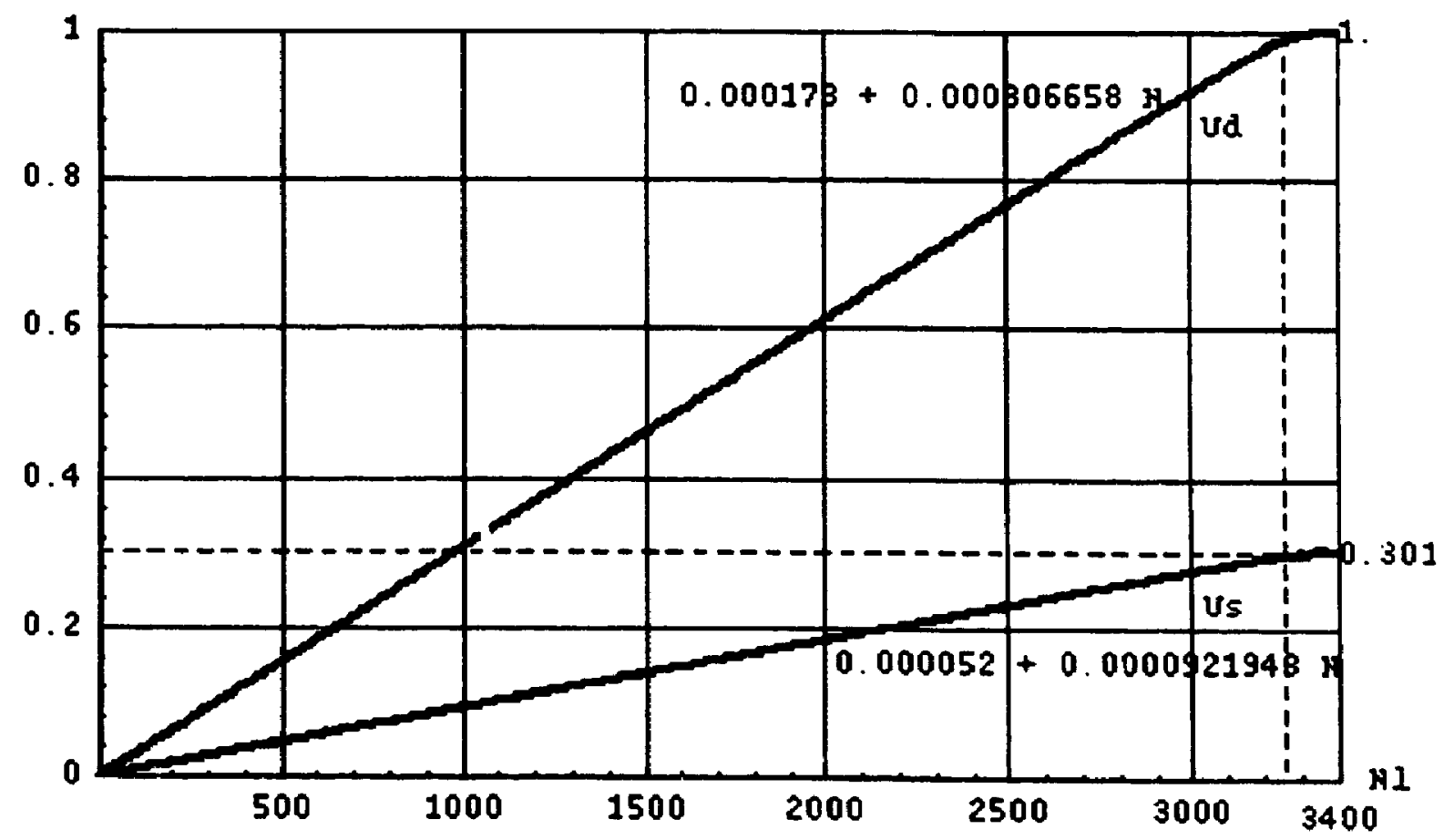

Figure 5 5.4. Utilizations of the server Us and disk Ud, vs the number of local users Nl with remote arrival rate 0.001 
Since the remote arrival rate is very small, the system can be considered as a closed model with only local users. The number of users, $\mathrm{N}$, at the intersection point of the components of the light load optimistic bounds and the heavy load bound is following [21 p.77],

$$
N^{*}=\frac{(D+Z)}{D \max }=\frac{(269+563757)}{173}=3260
$$

there, $D$ is the total demand of all the service centers; $Z$ is the thinking time of local users; Dmax is the largest demand among the queue service centers.

The light optimistic load and heavy load bounds of local response time of local requests are:

$$
\mathrm{Rl} \geq \mathrm{D}=0.269 \mathrm{~s}
$$

$$
\mathrm{RI} \geq(\mathrm{N} 1 * \mathrm{Dmax}-\mathrm{Z})=(0.173 * \mathrm{~N}-564) \mathrm{s}
$$

The remote response time is similar to the local response time except it has extra internet delay which is $0.358 \mathrm{~s}$ for a round trip. And the bounds of the remote response time would be:

$$
\mathrm{Rr} \geq(\mathrm{D}+\mathrm{Di})=(0 .-69+0.358)=0.627 \mathrm{~s}
$$

$$
\mathrm{Rr} \geq(\mathrm{N} 1 * \mathrm{Dmax}-\mathrm{Z}+\mathrm{Di})=(0.173 * \mathrm{~N} \mathrm{l}-564+0.358)=(0.173 * \mathrm{~N} \mathrm{-}-564) \mathrm{s}
$$

there, $\mathrm{Di}$ is the internet delay. 
The light optimistic load and heavy load bounds of throughput of local requests are:

$$
\begin{gathered}
\mathrm{Xl} \leq \frac{\mathrm{Nl}}{\mathrm{D}+\mathrm{Z}}=\frac{\mathrm{N} l}{0.269+564}=0.0398 \mathrm{I} * \mathrm{Nl} / \mathrm{s} \\
\mathrm{Xl} \leq \frac{1}{\mathrm{Dmax}}=\frac{1}{0.173}=5.78 / \mathrm{s}
\end{gathered}
$$

As we mentioned in lust section that the local system have about 100 workstations which are able to run window based WWW clients, which is much less than $\mathrm{Nl}^{*}=3260$ (this is because the thinking time of local user, $Z=564$ second, is so big). Therefore, the WWW system would always work in light load if there are not too many remote requests $(4.95 / \mathrm{seconds}$. for example, as assumed in section 6.3.2). The response times wouldn't be too big, no matter how many workstations are used to run WWW clients at the same time. Besides, if local users exceed $\mathrm{Nl}^{*}=3260$, the response time would grow fast. Therefore, the system can only work properly below $\mathrm{N} 1^{*}=3260$.

\subsubsection{Local User Performance with Remote Arrival Rate 3/second}

In this section, we will give the performance of WWW model for local user with remote arrival rate as $3 /$ seconds which is $3 / 5.78=52 \%$ of the system's saturation. Figure 6.6 .1 shows the response time of remote requests. $i_{2}+d$ all the performance figures, including response time, throughputs and utilizations are given in Figure D.5.1 to D.5.4 in Appendix D, in order to number of figures here. 


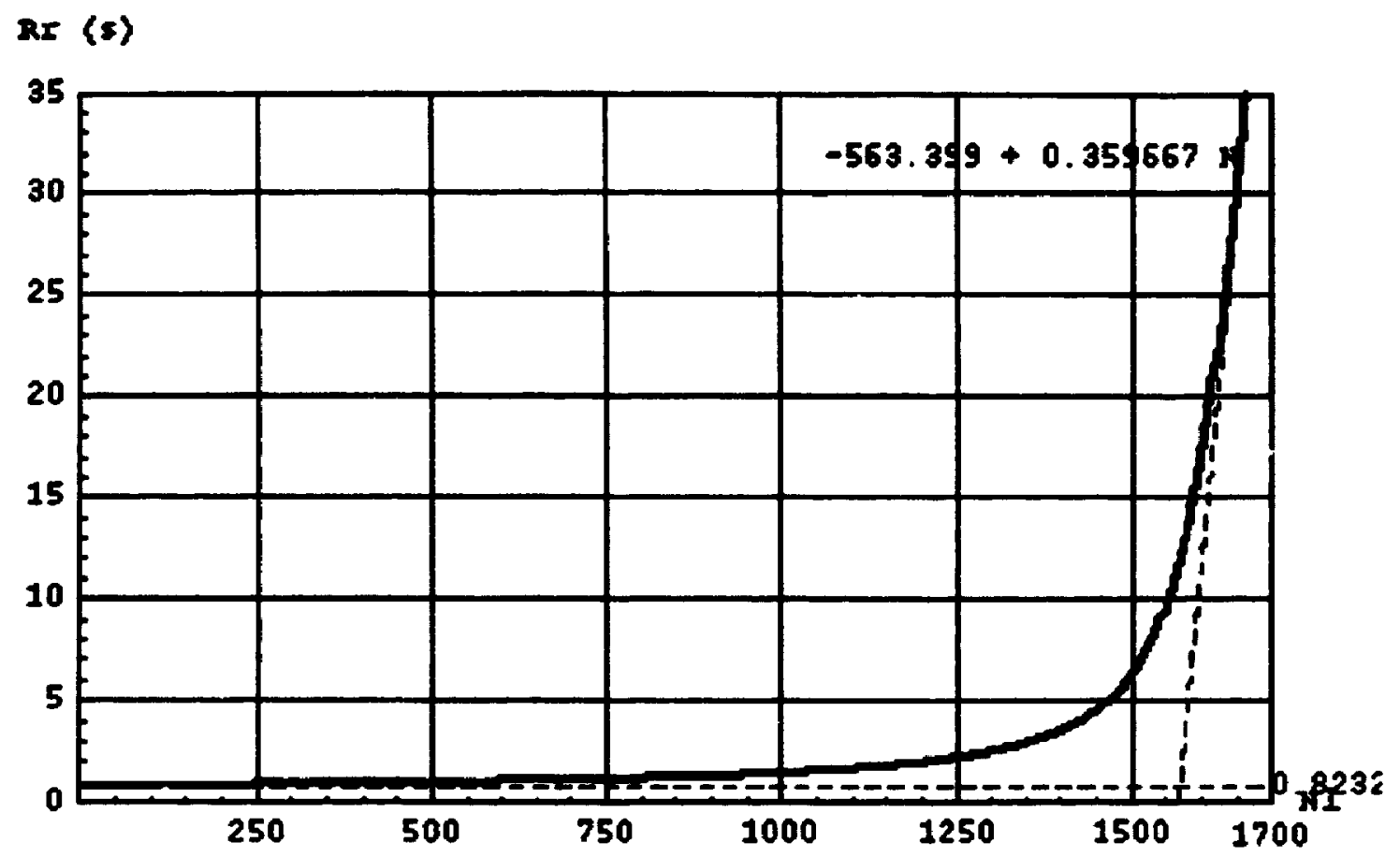

Figure 6.6.1. Response time of remote requests $\mathrm{Rr}$ vs the number of local users Nl, with remote arrival rate 3

When there are remote requests in the local user performance, part of utilizations of server and disk are taken by the remote requests. Since the remote arrival rate is a constant in this situation, we can solve this model by reducing the spoed of the server and disk as mentioned in 6.1. The new demands of server and disk are:

$$
\begin{aligned}
D^{\prime} s & =\frac{D s}{1-F^{*} D s}=\frac{52}{1-3^{*} 0.052}=62 \mathrm{~ms} \\
D^{\prime} d & =\frac{D d}{1-F^{*} D d}=\frac{173}{1-3^{*} 0.173}=360 \mathrm{~ms}
\end{aligned}
$$

The number of users, $\mathbf{N}$, at the intersection point of the components of the light load optimistic bounds and the heavy load bound $\mathrm{N}^{*}$ is

$$
\mathrm{Nl}^{\prime *}=\frac{\left(\mathrm{D}^{\prime}+\mathrm{Z}\right)}{\mathrm{D}^{\prime} \max }=\frac{(466+563757)}{360}=1567
$$


The light optimistic load and heavy load bounds of local and remote response time are::

$$
\begin{gathered}
\mathrm{Rl}^{\prime} \geq \mathrm{D}^{\prime}=0.466 \mathrm{~s} \\
\mathrm{Rl^{ \prime }} \geq\left(\mathrm{N} \mathrm{I}^{*} \mathrm{D}^{\prime} \max -\mathrm{Z}\right)=(0.360 * \mathrm{Nl}-564) \mathrm{s} \\
\mathrm{Rr}^{\prime} \geq\left(\mathrm{D}^{\prime}+\mathrm{Di}\right)=(0.466+0.358)=0.824 \mathrm{~s} \\
\mathrm{Rr}^{\prime} \geq\left(\mathrm{N} \mathbf{l}^{*} \mathrm{D}^{\prime} \max -\mathrm{Z}+\mathrm{Di}\right)=(0.360 * \mathrm{Nl}-564+0.358)=(0.360 * \mathrm{Nl}-563) \mathrm{s}
\end{gathered}
$$

The light optimistic load and heavy load bounds of throughput of local requests are:

$$
\begin{gathered}
\mathrm{Xl} \leq \frac{\mathrm{l}}{\mathrm{D}^{\prime} \max }=\frac{1}{0.360}=2.78 / \mathrm{s} \\
\left.\mathrm{XI}^{\prime} \leq \frac{\mathrm{Nl}}{\mathrm{D}^{\prime}+\mathrm{Z}}=\frac{\mathrm{N} l}{0.466+563.758}=0.0177 * \mathrm{~N}\right] \mathrm{s}
\end{gathered}
$$

Because of the additional remote request workload in the local system, $\mathrm{Nl}^{*}$ becomes $1567 /$ $3260=48 \%$ of the case when there only local users in the system. Also, the response time becomes bigger. And the increment of the response time becomes more significant when the system works in heavy load as shown in Figure 6.7.1. We can see. from Figure 6.7.1, when the system work in heavy load, $\mathrm{Nl}>\mathrm{Nl}^{*}$ for example, the impact of the local users on the system would be significant.

Again, the system is stable even though utilization and throughput of disk reach to their saturations in the heavy load (refer to Figure D.5.1 to D.5.4). For example, the utilization of disk becomes 1 when the number of local users is 1686 , and the response time of remote requests is 43 si:conds. Together with the situation in section 6.3.2, the stability of the system only depends on the remote arrival rate. The system is stable before the remote arrival rate reaches its saturation regardless how many local users are in the system. However, if we consider the reality of the response time, the system shouldn't working in very heavy load. For example, 
$\operatorname{Rr}(100)-\operatorname{Rr}(1)(s)$

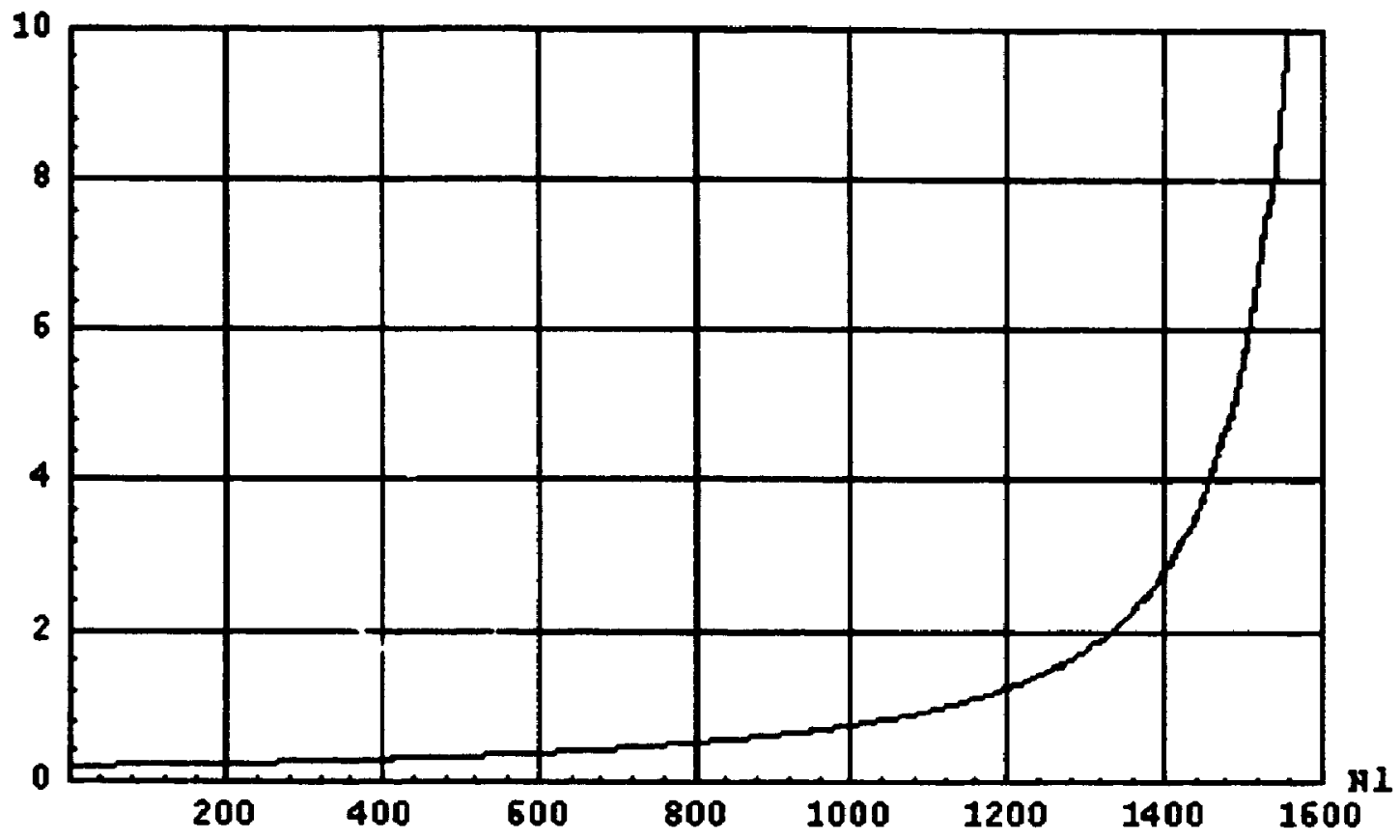

Figure 6.7.1. The difference of remote response time of remote arrival rata 0.001 and 3 vs the number of local users

when remote arrival rate is $5.5 /$ second and the number of local users is 500 , the remote response time would be 1220 seconds. Most users wouldn't wait for so long to retrieve the files.

More performance analysis of the system is shown in Figure D.2.1 to D.2.4. in Appendix D, with remote arrival rate $5 /$ second.

With all the situation we present so far, we can find that the total throughput in which the system could work properly is roughly less than $4.95 / \mathrm{s}$. Otherwise, the response times would grow dramatically even with little more workload.

\subsection{Some Issues About the Parameters and Performance}

One of the issues is that the Local User Delay time is large, as we mentioned before. This is 
very different from the normal thinking time of normal users. Actually, the user thinking/ reading time is about 37 seconds as reported in Table 5.3 and Figure 5.3. However, the Local User Delay time of our model is the total delay between events when the local user requests the local server. In other words, the Local User Delay time is large because it includes a lot of remote operations and other desktop operations. And just because it is large, local users doesn't significantly affect the performance of WWW model.

Another issue we need to mention here is the internet delay. Since we got the delay time from NSFNET Backbones which is only one backbone of the United States, the delay time used in our WWW model seems smaller than it should be. In fact, many WWW traffics are across countries. The delay time also varies in different time of the day. Sometimes it even taken hours to load a file at the rush hour. On the other hand, we had some assumptions in section 6.2 when computing the internet delay. Some researchers suggests that the internet delay of packet is about $100 \mathrm{~ms}$. Reliable statistics on internet delay time are lacking. Fortunately, the internet delay time does not affect our model significantly. When the internet delay time is changed, we can just add the change to the response time of remote requests. The other metrics will keep the same as they were.

The third issue is the file size with the server, which is 7646 in average. This could be very different between different servers. And the changing of the file size affects most of the demands of the model, especially the demands of the server and disk. Therefore, the performance of WWW system would be very different. This issue will be discussed in more detail in next chapter.

\subsection{Summary}

A queuing network model of WWW system with a surrogate server was presented in this chapter.

The parameters needed in this model are found from measurement or from some statistical data. The model is solved using Qnap2 package with both remote requests as an open class 
and local user requests as a closed class. The response times, throughputs and utilizations are given in 4 different ways. With this model, the maximum total request throughput is $5.78 / \mathrm{sec}$ ond. The system would work well with the total request throughput less than about $4.95 /$ seocnd even up to 100 local users are active (since they have little impact). The bottle neck of the system is disk.

The method of modelling in thesis chapter could be used for different WWW servers by just change the value of some parameters since the parameters could be very differences between servers. 


\section{Chapter 7}

\section{The Influence of System Parameters on Performance of WWW System Model}

There are thousands of WWW servers running in different systems. The system parameters between the different servers are all different. In this chapter, some of issues with changing of parameters will be discussed first. Then we will see the effect on response times of requests with different file size and different

\subsection{The Performance With Different File Request Sizes.}

The file sizes with different servers could be very different. Since the disk is a bottleneck in our model, a heavier file request demand per response will cause saturation at lighter loads. Intuitively, a doubling of the size of a retrieval will cut the capacity significantly, since the change of file size in a request will change the disk demand.

The baseline model in Figure 6.1 has an average retrieval size of 7646 bytes. Assuming a packet size of 512 bytes as discussed in section 6.2, this is $\mathrm{PK}=7645 / 512=15$ packets. To consider other values of PK, the parameters in Table 6.1 were assumed to be incurred per packet (this is clearly true for the disk, which is the key resource). That is, they were scaled by the factor of $(512 / 7646)$, as following:

Demands per packet $=(512 / 7646) *($ Demands in Table 6.1 $)$

From the logging data of the measurement with Mosaic, we found that the maximum file size which had been loaded in the measurement of Mosaic was $14,408,388$ bytes. With 512 bytes per packet, the range of files size is from 0 to 28142 packets. We keep the ratio of remote request throughput and the local user request throughput as about 10:1, and give the remote 

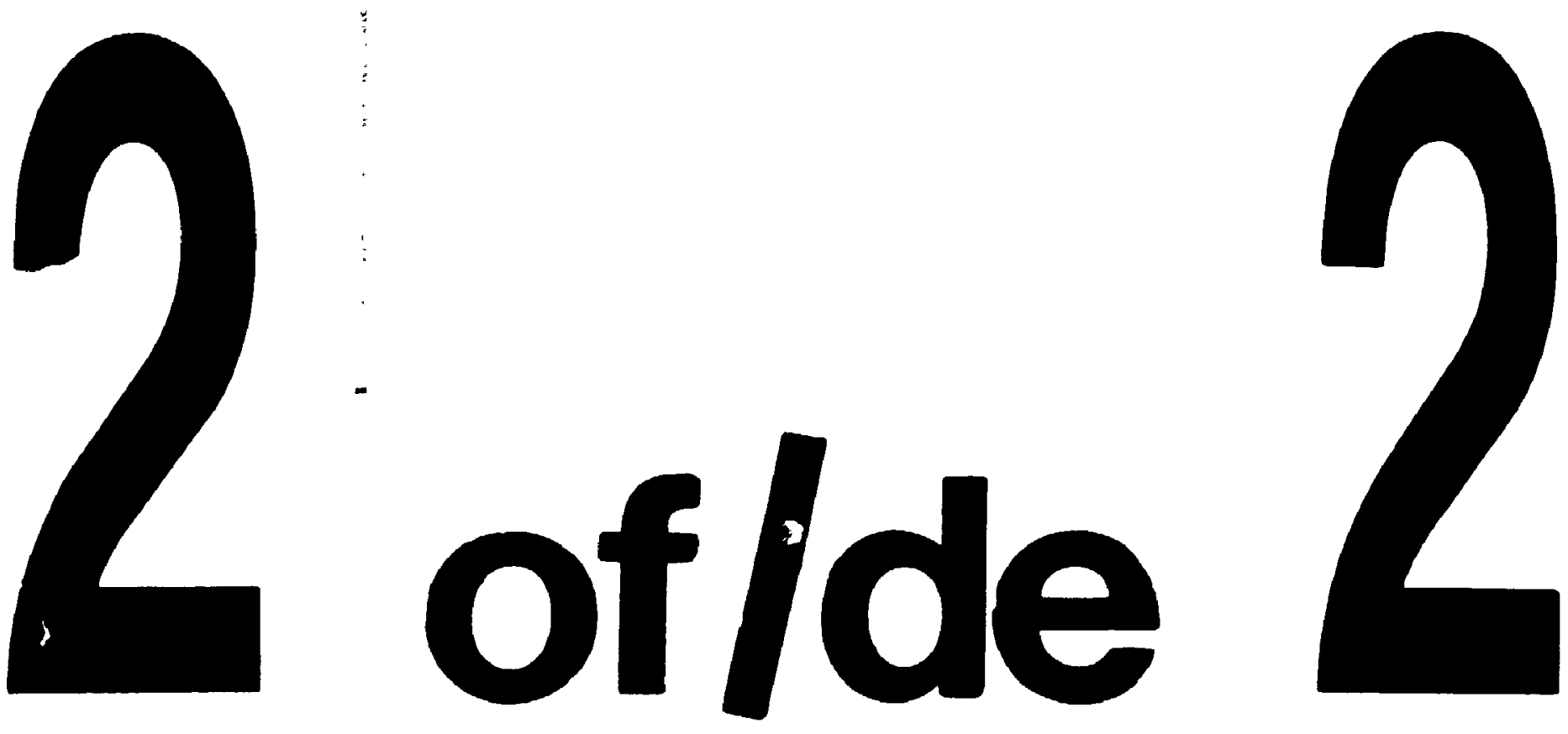

PM-1 31/"X" PHOTOGRAPHIC MICAOCOPY TARGET NES 1010a ANSI/ISO "2 EOUIVALENT

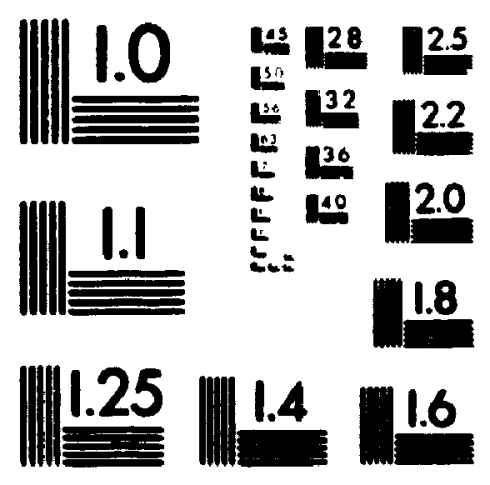

PAECISIONEM RESOLUT. EN TARCETS 
response times vs the number of packets of a file in different cases, as shown in Figure 7.1.1 in which the remote arrival rate $F r$ is 0.5 with 28 local users. and in Figure 7.1.2, in which Fr is 1.77 with 100 local users.

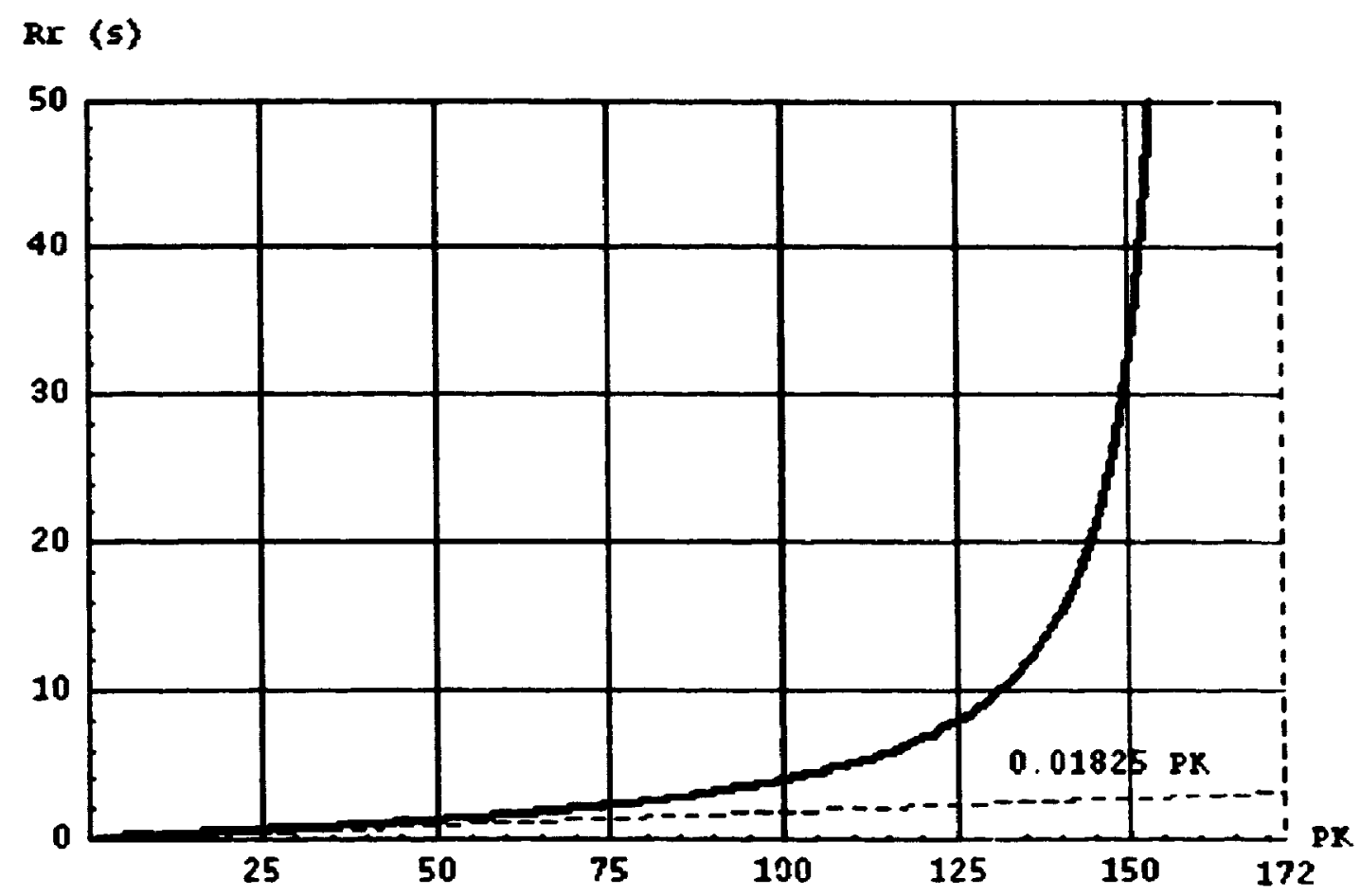

Figure 7.1.1. Response time of remote requests $\operatorname{Rr}$ vs the number of packets of a request $\mathrm{PK}$, with 28 local users and remote arrival rate 0.5 


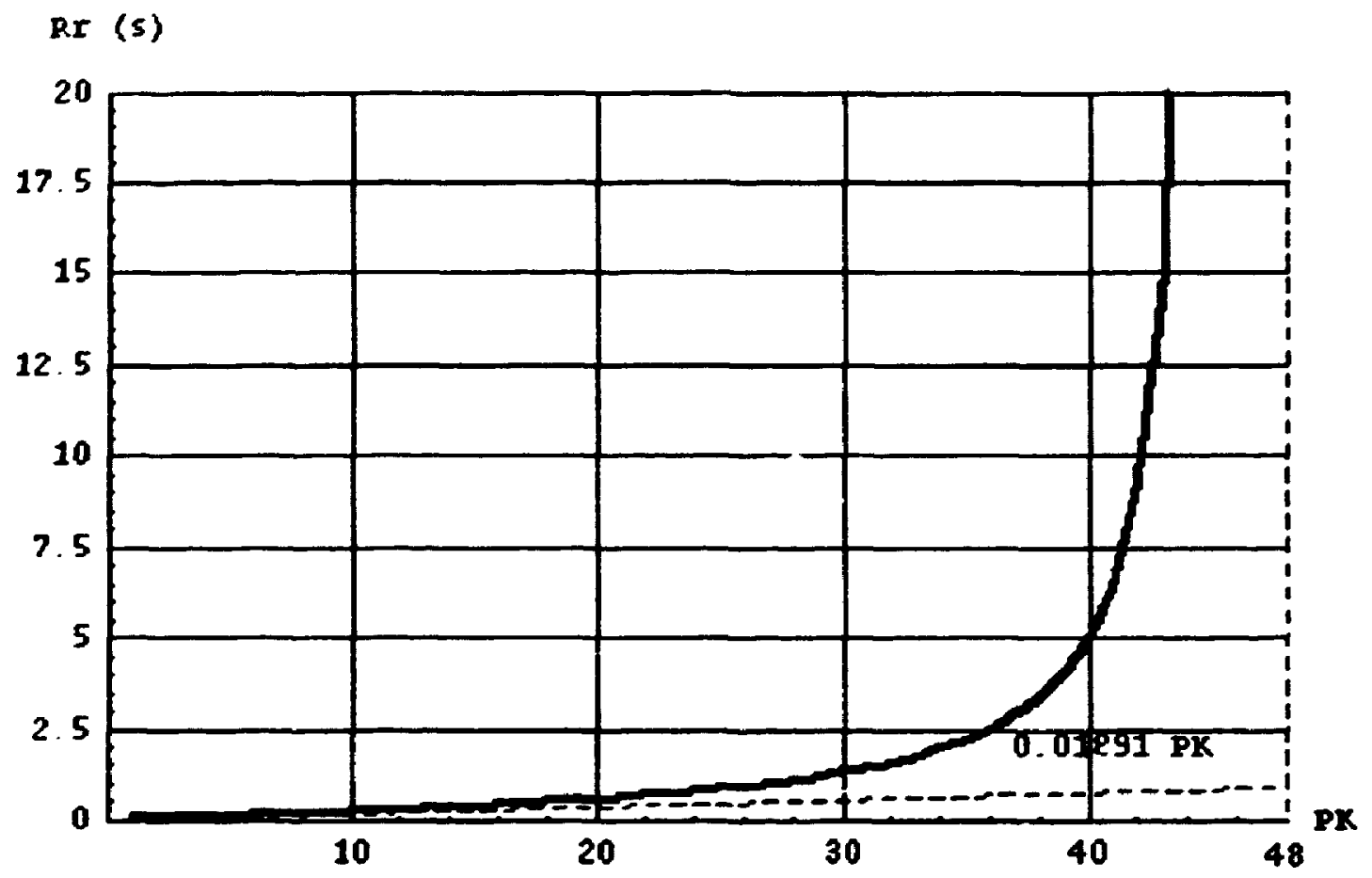

Figure 7.1.2. Response time of remote requests $\operatorname{Rr} v$ s the number of packets of a request PK. with 100 local users and remote arrival rate 1.77

From the figures. we can see that there is a saturation with an increasing number of packets. We already know, from the performance analysis in Chapter 6 , that when the system reaches its saturation, the local request throughpui goes to zero so that the total throughput of the system Xs is the same as the remote arrival rate Fr. And since the disk is the bottleneck, then vie have the following analysis of the saturation:

$$
F_{r}=\frac{1}{\text { Dmax }}=\frac{1}{(\text { Demand per packet of disk }) \times(\text { Number of packets })}
$$

Then for a given Fr, we have the system saturation when PK=PK*:

$$
\mathrm{PK}^{*}=\frac{1}{(\text { Demand per packet of disk }) \times \mathrm{Fr}}=\frac{1}{\left(0.173^{*} 512 / 7646\right) \times \mathrm{Fr}}
$$

In Figure 7.1.1. Fr is 0.5. and the packet saturation PK* is 172. Although the system is stable 
before the saturation, the response time would be too big and grow too fast. Again, with maximum useful remote response time as 2 seconds, as assumed in section 6.3. the packet number is limited to less than 70 . In other words, the system could work properly when the number of packet is about less than 70. But with more requests, the saturation of PK would be smaller. as shown in Figure 7.1.2, in which the remote arrival rate Fr is 1.77 and so the sasuration of packets is 48. In this case, the packet number is at the point of remote response time as 2 seconds. It is better to keep the packet number less than 34 .

Therefore, the file size is important parameter for the system performance. The more requests to the system, the smaller the size of the file should be. This can be seen again in Figure D.3.1 with much more heavy load.

The lines $0.01825^{*} \mathrm{PK}$ in Finger 7.1.1 and $0.01891 * \mathrm{PK}$ in Figure 7.1 .2 give a brief idea about how fast the response times increase with PK when it is small. Although they indicate that $\mathbf{R r}$ increase faster with more requests in Figure 7.1.2 than with less requests in Figure 7.1.1 because 0.01891 is bigger than 0.01825 , the difference is small.

\subsection{Effect of Caching on The Performance}

As we already see from the previous analysis, the bottleneck of the system is the disk. One of the ways to improve the performance of the system is caching. Suppose the server workstation has enough memory, and some of the files stay in the memory cache. When a file, which is in cache memory, is requested for access, the server does not need to access the file from the disk, so that the demand of disk is reduced. Therefore, the performance of the system will be improved.

Let the original disk demand be $\mathrm{Dd}$, and the caching hit ratio is $\mathbf{C}$ which is the ratio of requested from cache memory and the total requests. Then the new disk demand with caching, $J " d$, is reduced by the same percent as the cache hit rate:

$$
D " d=(1-C) \times D d
$$


And then, the saturation of the remote arrival rate $F^{\prime \prime} r^{*}$ is also changed (increased) as the same percent as the cache hit ratio from its basic saturation Fr*:

$$
F^{\prime \prime} r^{*}=\frac{1}{D \max }=\frac{1}{D^{\prime \prime} d}=\frac{1}{(1-C) \times D d}=\frac{1}{(1-C)} \times F^{*}
$$

This indicates that the system capacity is also improved by

$$
\frac{\mathrm{Fr}^{*} \mathrm{r}^{*} \mathrm{Fr}^{*}}{\mathrm{Fr}^{*}}=\frac{\mathrm{C}}{\mathrm{I}-\mathrm{C}}
$$

Suppose the caching hit ratio $\mathrm{C}$ are $\mathbf{0 . 1}, 0.2$ and 0.3 . Then the new disk demands $\mathrm{D}^{\text {"d }} \mathrm{d}$ and the Saturation of remote arrival rate $F^{\prime \prime} r^{*}$ with caching are listed in Table 7.1

Caching hit ratio Disk Demand Saturation of Remote Arrival Rate
0
$173 \mathrm{~ms}$
$5.780(1 / 5)$
0.1
$155.7 \mathrm{~ms}$
$6.423(1 / s)$
0.2
$138.4 \mathrm{~ms}$
$7.225(1 / \mathrm{s})$
0.3
$121.1 \mathrm{~ms}$
$8.258(1 / s)$

Table 7.1. Disk demands and saturation of remote arrival rate with caching

We re-solve the WWW model in section 6.3.2 with the same parameters and with caching. The performance of the model in four different cases, in which the caching hit ratio are $C=0$, 0.1, 0.2 and 0.3 respectively, are shown in Figure D.6.1 to D.6.4 in Appendix D to order to reduce the number of figures in this chapter. Here we only show the performance of remote response time in Figure 7.2.1. 


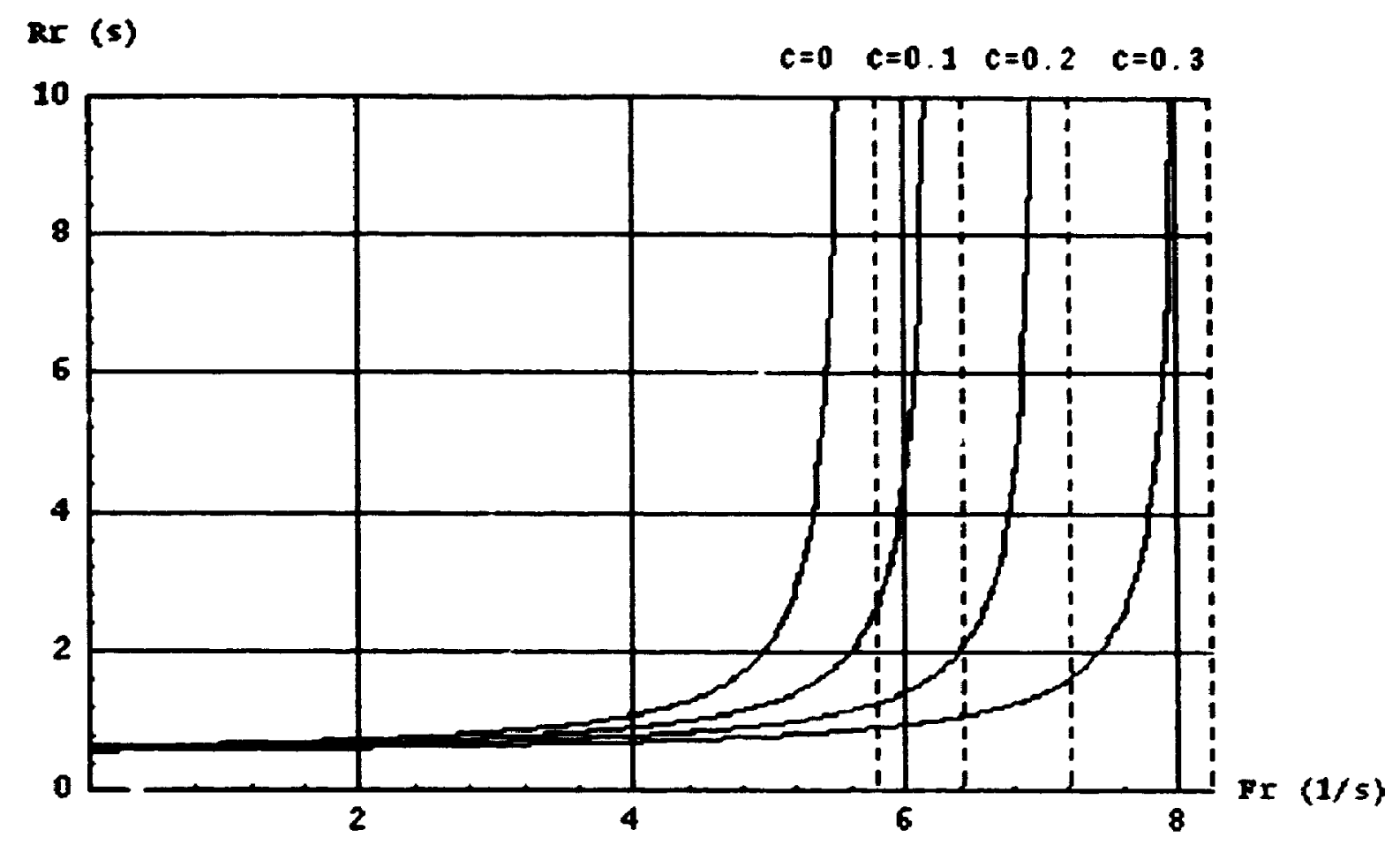

Figure 7.2.1. Response time of remote request $\operatorname{Rr}$ vs remote arrival rate $\mathrm{Fr}$, with 100 local users and caching hit ratio $C=0,0.1,0.2,0.3$.

It is obviously that the performance is improved, especially when the system is in heavy load. The bigger the caching hit ratio, the more improvemen' the performance. The improvements of the saturations of remote arrival rate increase about $11 \%, 25 \%$ and $43 \%$, respectively with caching hit ratios as $0.1,0.2$ and 0.3 respectively (refer to Table 7.1 ).

Again, we assume the maximum useful remote response time is 2 seconds. From Figure 7.2.1, we can see that the system can only work properly when the remote response time is less than 2 seconds. When the remote response time is larger than $\mathbf{2}$ seconds, it would grow dramatically with little more requests. At this point, the performance metrics are shown in Table 7.2. 


$\begin{array}{llllll}\text { C } & 0 & 0.1 & 0.2 & 0.3 & \\ \text { Rr (remote response time) } & 2 & 2 & 2 & 2 & \text { (s) } \\ \text { RI (local user response time) } & 1.642 & 1.642 & 1.642 & 1.642 \text { (s) } \\ \text { Fr (remote arrival rate) } & 4.95 & 5.59 & 6.39 & 7.42 \quad(1 / s) \\ \text { Xs (total throughput) } & 5.127 & 0.767 & 6.567 & 7.587 \\ \text { XI (local request throughput) } & 0.1769 & 0.1769 & 0.1769 & 0.1769 \\ \text { Us (server utilization) } & 0.2666 & 0.3 & 0.3415 & 0.395 \\ \text { Ud (disk utilization) } & 0.887 & 0.8981 & 0.9088 & 0.92\end{array}$

Table 7.2 The performance metrics at the pointer of $\mathrm{Rr}=\mathbf{2}$ seconds, local users is 100 , with caching hit ratio $\mathrm{C}=0,0.1,0.2$ and 0.3 .

From Table 7.2, we can see that most of the performance metrics are improved with caching, except the local user request throughput. The reason that there is no improvement shown on the local user request is that the Local User Delay time is larger, so that the users stay in their workstations without requesting the server for most of the time. The utilizations of server and disk are larger with caching. Together with the improvement of the throughput, this indicate that the system work more efficiently with caching even though we keep the same response time.

In summary, the improvements of the system with caching compared with no caching are listed in Table 7.3. They are shown either in the saturation or in the point of 2 seconds of remote response time.

In table 7.3. $\mathrm{C}$ is the caching hit ratio, $\mathrm{Fr}^{*}$ imp is the improvement of the saturation of remote arrival rate, $\mathrm{Xs}^{*}$ _imp is the improvement of the maximum total request throughput. The improvements of other performance metrics are given at the point when remote response time is $\mathbf{2}$ seconds. as we assumed before. At this point, we define $\mathbf{F r} \mathbf{R r}$ _imp is the improvement of remote arrival rate; $X s_{-} i m p$ is the improvement of total request throughput; $X l \_i m p$ is the improvemen: of local user request throughput. Us_imp is the improvement of the server, Ud_imp is the improvement of disk. 


$\begin{array}{llll}\text { C } & 0.1 & 0.2 & 0.3 \\ \text { Fr*_imp } & 11 \% & 25 \% & 43 \% \\ \text { Fr_Rr_imp } & 13 \%, & 29 \% & 50 \% \\ \text { Xs_imp } & 12 \% & 28 \% & 48 \% \\ \text { Xs*_imp } & 11 \% & 25 \% & 43 \% \\ \text { XI_imp } & 0 \% & 0 \% & 0 \% \\ \text { Us_imp } & 13 \% & 28 \% & 48 \% \\ \text { Ud_imp } & 1 \% & 3 \% & 4 \%\end{array}$

Table 7.3 Improvement of the WWW system with caching, comparing to the case in section $\mathbf{6 . 3 . 2}$

We don't show the improvement for the response time of local user requests because it is similar to the one for the response time of remote requests except the local response time is smaller by the amount of the internet delay than remote requests

\subsection{Summary}

The WWW servers in the internet are different from the one we used for measurement and analysis. Therefore, the performance would be also difterent with different parameters. The most sensitive parameter is the file size to be transferred, to which the demand of the model is related. With an increase of the file size, the performance of the system becomes worse. Caching technique is frequently used to reduce the access to disk. With high caching hit rate, the performance of the system is improved significantly. 


\section{Chapter 8}

\section{Conclusions and Future Studies}

\subsection{Conclusion}

World Wide Web is a powerful and popular information exchange system in the internet. Its use has been increasing dramatically, and it has become the number 1 traffic in the network back bone. Therefore, WWW applications became a major workload component of network and computer systems. The performance analysis of $W W W$ system is necessary. The results of $W W W$ performance study will be useful for design and improvement of WWW software, and useful for system planers.

This thesis focuses on measurement and analysis of World Wide Web system using both measurement and analytic modelling. Although measurement is the most costly technique, it is necessary to use it in order to find the system parameters, and to find the behavior of user ind program. The analytic modelling would give a brief, quick view of the performance of WWW system.

Experimental versions of Mosaic and Lynx were implemented, including software event monitor. This version of Mosaic was run in the local system for 29 days. The data measured are collected either through e-mail or logging files. $C$ and Mathematica programs were written to process and present the data. System accounting monitor is used for measuring the WWW server in our local system. With the built-in logging function of the server, most of the parameters needed in the study are obtained. Other data were obtained either from internet statistics and from our other experiments.

One of the major issue studied is the user behavior and workload characterization of the client. Normally, the clients and servers of WWW system are in different sites of the internet, so 
the client and server are different components of the workload. Because the WWW clients are user interactive software, the user behavior is the important issue. Mosaic client are chosen as a case in this thesis because Mosaic is a typical graphic window-based WWW client. A User Behavior Graph [15] model is used to describe the performance of Mosaic. This model can provide additional information about the workload characterization, user behavior and program performance, although it is not neces_ary for the performance model of WWW system which was built in Chapter 6. In the Mosaic model, most of the user input and some of important program events are described as transition. The completions of actions are states. With the measured data, the model is solved with both the transition probabilities and the system resource utilizations for different states. Cpu time, user reading time, file loading time and file size are described in more detail. Loading file is the most frequent action of the users and is the most system resource consumed state in the model.

Although some researches in WWW clients and user behavior had been done, the model of Mosaic client we built is the first one of WWW client. This model provides more information about the user behavior, program performance and workload parameters.

The Queuing Network Model of WWW system is built. The server measured and modeled in the model is the WWW server in the System and Computer Engineering Department. This is the first full-scale performance model of an entire WWW system. In this model, the requests may be either from the remote users or from the local users. The parameters of model are from our measurement, the internet statistics and from our other experiment. The performarce metrics presented are response time, throughput and utilization. The remote requests and local requests are inputs of the sy stem. Other issues studied include the performance with the different file sizes and the effect of caching on the performance.

We found, from the model, that the WWW server in the System and Computer Engineering Department at Carleton University could accept about 5 requests/second. under present circumstances, and would not be very sensitive to the number of local users. The bottleneck is the server disk, and the performance is very sensitive to the size of files retrieved, presently averaging about $7 \mathrm{~K}$ bytes with the server. The file size the server provides has significant 
impact on the system performance. A doubling in file size would reduce request capacity by half. Thus the requests to graphics file will affect system performance, since graphics file are large. The caching technique can improve the system performance. With caching hit ratio $50 \%$, the system capacity can be as 2 times as the original system.

The model study in chapter 6 and chapter 7 has some limitations. It does not say much about a server which is configured differently from this department's server, although it seems that the server model could be scaled. The representation of Internet delay is based on reported measurements which may not be representative.

\subsection{Further Studies}

To continue the study following the thesis work in the future. Some of them are:

(1) Set distributed WWW clients with Decal and for artificial users

The measurement of WWW applications needs real users. The new version of WWW client application with artificial users would be a choice. Actually, a automatic execution version of Lynx is implemented for the purpose of system performance study. There is no user needed to run it. Instead, there is an artificial user function implemented in the with the original program. The measurement could be done without users so that more lynx processes could be set on the network to get more experiment data. With the measurement tool for distributed application DECALS [40] together, different number of Lynx process could be set on the network automatically, and the events could be monitored, and useful data could be save in logging files for system performance study. But that version of Lynx was done before the User Behavior Graph is done so it did use the parameters we measured. Also, automatic versions of Mosaic or Netscape can be implemented to get the measurement data without users. With this distribured and artificial user version of WWW clients, system performance and be measured with the assigned workload. These measurement could be compared to the performance of WWW model in Figure 6.1. Some other measurement tools could be employed because current version of DECALS has problem deal with many graphic window applications. 
(2) Build more precise User Behavior Graph Model

In the User Behavior Graph model of Mosaic in Figure 4.1, some of states hide the detailed user actions. For example, the "Hotlist" state has more user actions inside of it. The "Hotlist" window is popped up when the "Hotlist" button is pushed. The user actions could be "add". "deleted". "edit" and so no. All of the actions could be modeled as a submodel of "Hotlist" state. This submodel is needed for implementing the automation versions of WWW client applications because the next button to selected depending on the previous button sometime.

(3) Measurement and Analysis of WWW Server Side

The measurement of the server in the thesis is taken with system accounting and built-logging function. These could not give much detail information about what is happening inside of the server. For example, the distribution, the confidence interval and other information are not obtained. For the purpose of improvement of server software and system planning, more detailed measurement and analysis work should be done with the server side. And with more data of the network, all the measurement of client and server would be used to make excellent performance model of WWW system. And the results of the performance study would be more precisely and more useful. 


\section{Reference}

[1] Tim Berners-Lee, Robert Cailliau, Jean-Fracois Groff, and Bernd Pollermann, WorldWide Web: The Information Universe, Electronic Networking: Research, Application and Policy, vol 2, No. Spring 1992, p.52-58.

[2] Tim Berners-Lee, Robert Cailliau, Ari Luotonen, Henrik Frystyk and Arthur Secret, The World-Wide Web, Communications of the ACM, 1994, vol. 37, No. 8, P.76-82

[3] Tim Berners-Lee, Robert Cailliau and Jean-Fracois Groff, The World Wide Web, Compunr Networks and ISDN Systems, Nov. 1992, vol 25, p.454-459

[4] NSFNET performance statistics: gopher://nic.merit.edu:7043/1/nsfnet/statistics

[5] National Center for Supercomputing Applications, A beginner's Guide to HTML, http:// www.ncsa.uiuc.edu/General/Internet/wWw/HTMLPrimer.html.

[6] Michael Grobe, The University of Kansas, HTML Quick Reference, http://www.ncsa.uiuc.edu/General/Internet/WWW/HTMLQuickRef.html.

[7] Dave Raggett, HyperText Markup Language Specification 3.0, http://www.w3.org/hypertext/WWW/MarkUp/html3/CoverPage.html, http://www.w3.org/hypertext/wWW/ MarkUp/html3/html3.txt.

[8] T. Berners-Lee, R. Fielding, H. Frystyk, HYPERTEXT TRANSFER PROTOCOL ․ HTTP/ 1.0, INTERNET-DRAFT, <draft-ietf-http-v10-spec-03.html>, September 4, 1995, http:// www.ics.uci.edu/pub/ietf/http/draft-ietf-http-v10-spec-0, http://www.ics.uci.edu/pub/ietf/ http/draft-ietf-http-v11-spec-00.ps.gz

[9] M. Andreessen and E. Bina, NCSA Mosaic: A Global Hypermedia System, Internet 
Research, Vol. 4 No. 1. Spring 1994 pp. $7-17$ ” 1994 Mecklermedia.

[10] University of Kans3s, About Lynx. http://www.cc.ukans.edu/about_lynx, 10/25/95

[11] Raj Jain, The Art of Computer Systems Performance Analysis Technique's for Experimental Design, Measurement, Simulation, and Modeling, John Wiley \& Sons, Inc., 1991.

[12] Liba Svobodova, Computer Performance Measurement and Evaluation Methods: Analysis and Applications. American Elseview Publishing Company Inc. 1976.

[13] Phillip McKerrow, Performance Measurement of Computer Systems, Addison-Wesley Publishing Company, 1987

[14] J.Pasquale, B.Bittel and D.Kraiman, $A$ Static and Dynamic Workload Characterization Study of the San Diego Supercomputer Center Cray X-MP, in: Proc. 1991 ACM Sigmetrics Conference on Measurement and Modeling of Computer Systems, San Diego, California, USA, Vol. 19 No.1 218-219.

[15] D. Ferrari. On the foundations of artificial workload design. in: Proc. 1984 ACM Sigmetrics Conf. on Measurement and Modeling of Computer Systems, Cambridge. MA (1984) 8-14.

[16] Maria Calzarossa and Domenico Ferrari, A Sensitivity Study of the Clustering -Approach to Workload Modeling, Performance Evaluation 6 (1986) p25-33, North-Holland.

[17] Philip Heidelberger and Stephen S. Lavenberg, Computer Performance Evaluation Methodology, IEEE Transactions on Computers, Vol. c. 33, No. 12, December 1984

[18] John F. Meyer, Performability: a retrospective and some pointers to the future, Performance Evaluation, 14(3\&4), pp.139-156. 
[19] Kishor S. Trivedi and Boudewijn R. Haverkort, Andy Rindos and Varsha Mainkar, Techniques and Tools for Reliability and Performance Evaluation: Problems and Perspectives., Computer Performance Evaluation Modelling Techniques and Tools, Proceeding of 7th International Conference, Vienna, Austria, May 1994, Springer-Verlag, p. 1-24

[20] S. S. Lavenberg, A Perspective on Queueing Models of Computer Performance, Performance Evaluation, vol. 10, pp. 53-76, 1989.

[21] E. D. Lazowska, J. Zahorjan and G. S. Graham and K. C. Sevcik, Quantitative System Performance: Computer System Analysis Using Queueing Network Models, PrenticeHall Inc., Englewood Cliffs, N.J. 07632, 1984.

[22] Peter G. Harrison and Naresh M. Patel, Performance Modelling of Communication Networks and Computer Architectures, Addison-Wesley Publishing Company, England. 1993

1231 Patricia A. Jacobson and Edward D. Lazowska. Analyzing Queueing Networks with Simultaneous Resource Possession. Communications of the ACM, February 1982, volume 25.

124] Tad2. Murata. Petri Nets: Properties, Analysis and Applications, Proceeding of IEEE, Vol. 77, No. 4, April 1989

[25] Giovanni Chiola, Marco Ajmone Marsan, Gianfranco Balbo and Gianni Conte, Generalized Stochastic Petri Nets: A Definition at the Net Level and Its Implications, IEEE Transactions on Software Engineering, Vol. 19, No. 2, February 1993.

[26] C. M. Woodside, Throughput Calculation for Basic Stochastic Rendezvous Networks", Performance Evaluaciot. vol. 9, n. 2, pp. 143-160, April 1989. 
[27] C. M. Woodside, J. E. Neilson, D. C. Petriu and S. Majumdar, The Stochastic Rendezvous Network Model for Pefformance of Synchronous Client-Server-Like Distributed Software, IEEE Transactions on Computer, 1995 Jan. vol. 44 no. 1 pp. 20 - 34

[28] C. M. Woodside, J. E. Neilson, D. C. Petriu and S. Majumdar, A Guide to Rendezvous Network Model for Performance of Distributed Client-Server Software. SCE-91-22. Department of Systems and Computer Engineering, Carleton University, 1991

[29] C. M. Woodside, A 3-View Model for Performance Engineering of Concurrent Software, IEEE Transactions on Software Engineering, Sep. 1995, vol. 21. No. 9, p. 754-767.

[30] Adrian Cockcroft, Sun Performance and Tuning, Sun Microsystems, Inc. 1995

[31] Hans-Werner Braun, Kimberly Claffy, Web traffic characterization: an assessment of the impact of caching, proceeding of The Third International WWW Conference in Darmsadt, Germany, http://www.ncsa.uiuc.edu/SDG/IT94/Proceedings/DDay/claffy/ main.html

[32] Jeff Sedayao, "Mosaic Will Kill My Network!" -Study in Network Traffic Patterns of Mosaic Use, Proceeding of The Third International WWW Conference in Darmsadt, Germany, http://www.ncsa.uiuc.edu/SDG/IT94/Proceedings/DDay/sedayao/mos_traf_paper.html

[33] Lara D. Catledge and J. Pitkow, Characterizing Browsing Strategies in the World-Wide Web, Computer Networks and ISDN Systems, April, 1995, vol.27 No.6, p. 1065-1073, http://www.igd.fhg.de/www/www95/papers/80/userpatterns/UserPatterns.Paper4.formatted.html

[34] J. Pitkow and M. Recker, Results from the first World-Wide Web survey, Computer Networks and ISDN systems, vol. 27, no.2, p.243-254 
[35] J. Pitkow and M. Recker, Using the Web as a Survey Tool: Results from the Second WWW User Survey, Computer Networks and ISDN systems, April, 1995, vol. 27, No. 6, p. 809-822, http://www.igd.fhg.de/www/www95/papers/79/survey/survey_2_paper.html

[36] WWW document, A Beginner's Guide to URLs, http://www.ncsa.uiuc.edu/demoweb/urlprimer.html

[37] Ramez Elmasri and Shamkant B. Navathe, Fundamentals of Database Systems, The Benjamin/Cummings Publishing Company, Inc. 1989.

[38] W.Richard Stevens, TCPIIP Illustrated, Volume 1,Addison-Wesley Professional Computing Series, 1994

[39] Unix Include File: /usr/include/netinet/ip.h (IP_MSS and IP_MAXPACKET).

[40] T.Elgillani, A.Hubbard, C.Schramm and F.Sheikh, DECALS: Distributed Experiment Control Aand Loggin System, User's Guide and Reference Manual, Real-Time and Distributed Systems Group (RADS), Department of Systems and Computer Engineering, Carleton University, Carleton University. 


\section{Appendix A.}

State Occurrences and Their Probabilities of Occurrences in Mosaic Model

Total sessions: 115

state 1: start

state 2: loading

state 3: main_window

state 4: submit_form

state 5: open_URL

state 6: reload

state 7: home

state 8: active_anchor

state 9: back

state 10: forward

state 11: key_press

state 12: hotlist

state 13: add_hotlist

state 14: printing

state 15: saving

state 16: exit
Total state occurrences: 10844

occurrences: 115 probability: 0.010605

occurrences: 2423 probability: 0.223442

occurrences: 4153 probability: 0.382977

occurrences: 108 probability: 0.009959

occurrences: 147 probability: 0.013556

occurrences: 30 probability: 0.002767

occurrences: 10 probability: 0.000922

occurrences: 1859 probability: 0.171431

occurrences: 1282 probability: 0.118222

occurrences: 90 probability: 0.008300

occurrences: 91 probability: 0.008392

occurrences: 122 probability: 0.011250

occurrences: 48 probability: 0.004426

occurrences: 28 probability: 0.002582

occurrences: 223 probability: 0.020564

occurrences: 115 probability: 0.010605

Table A.1. State Occurrences Probabilities of in Mosaic Model 


\section{Appendix B.}

\section{Cpu Time Distributions of Mosaic Model}

The figures in this appendix are histograms to show the distributions of cpu time of the states in the User Behavior Graph model in Figure 4.1. The vertical axis is the number of occurrences felled in the cells. The number of cells is depended on the range of the cpu time. More cells for large range of cpu time. The horizontal axis is the number of cells of the sample cpu time data from minimum to maximum. The pair of number at the right end of horizontal axis is the range of cpu time, that is, (minimum, maximum) seconds. In Figure B.1 for state 1 (start), for example, the pair of number at the horizontal axis is $(1.34,3.76)$, which means the minimum cpu time is 1.34 second and the maximum cpu time is 3.76 seconds. The range of cup time is divided into 5 cells. About 30 samples of cpu tirne data felled into cell 1.

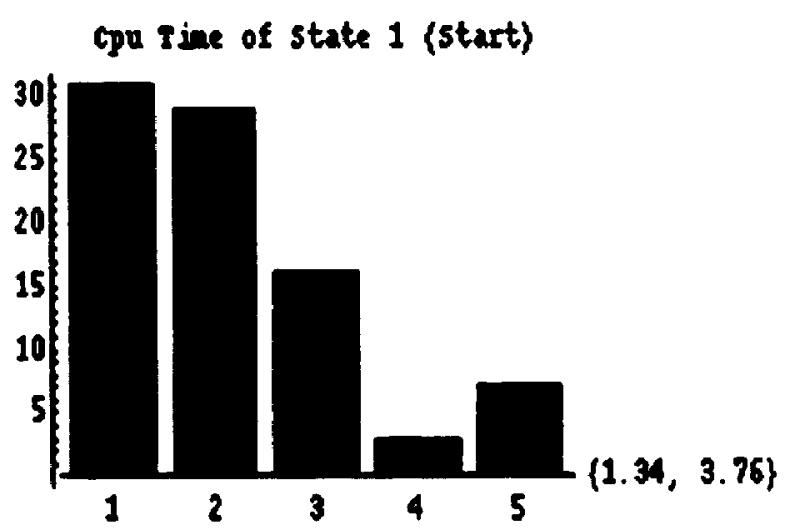

Figure B.1 Distribution of cpu time of "Start" state, 5 equal cells in the range $(1.34,3.76)$ seconds

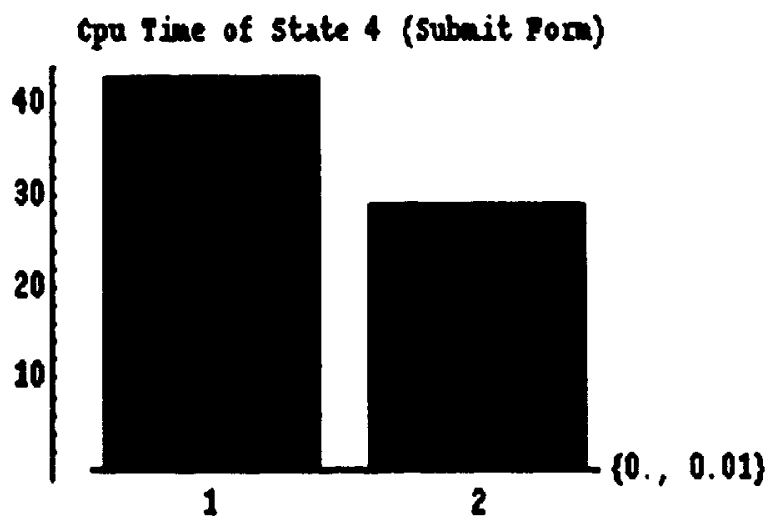

Figure B.4. Distribution of cpu time of "Submit Form" state, 2 equal cells over $(0,0.01)$ seconds 


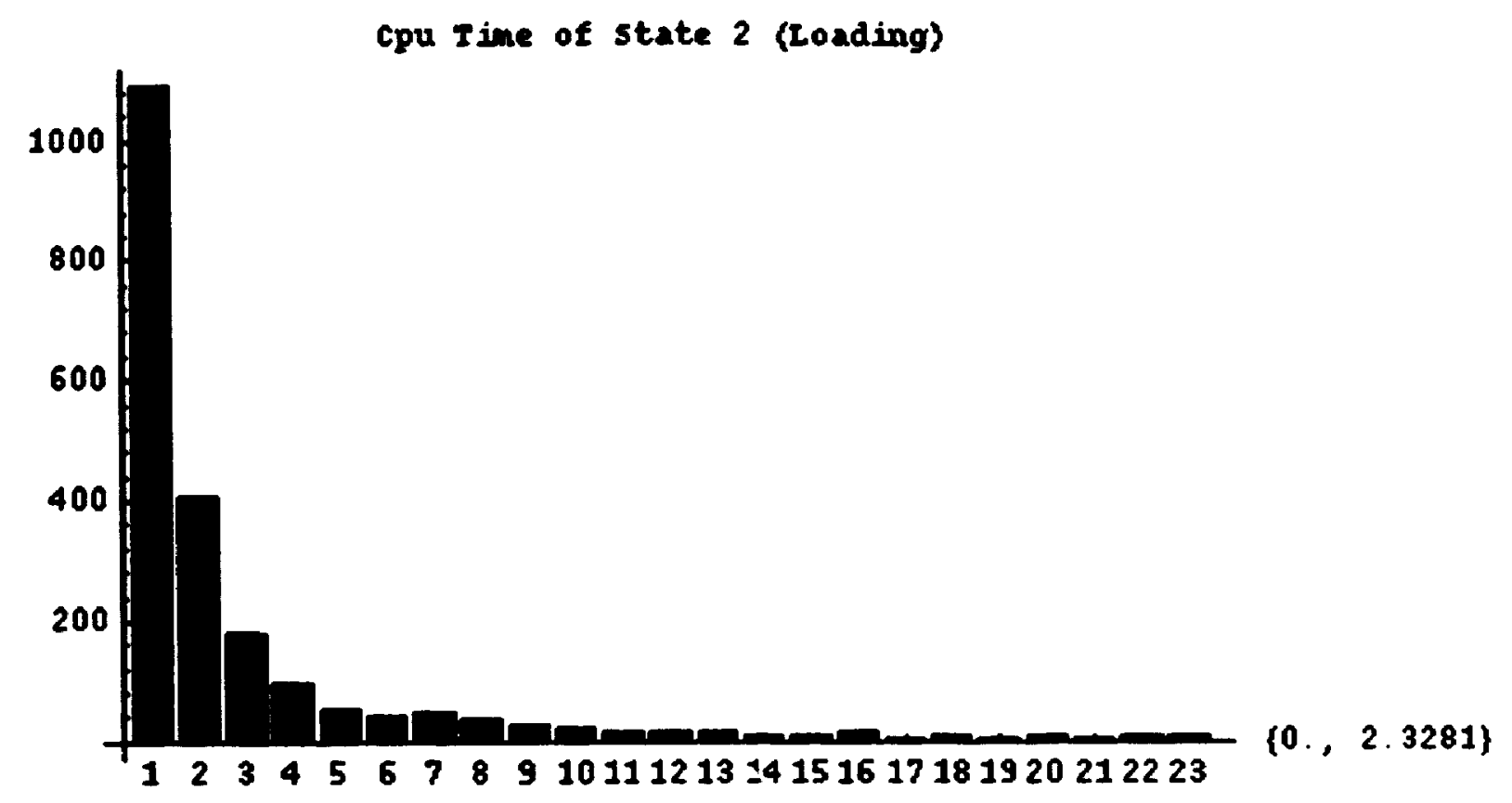

Figure B.2. Distribution of cpu time of "Loading" state, 23 equal cells in the range $(0,2.3231)$ seconds

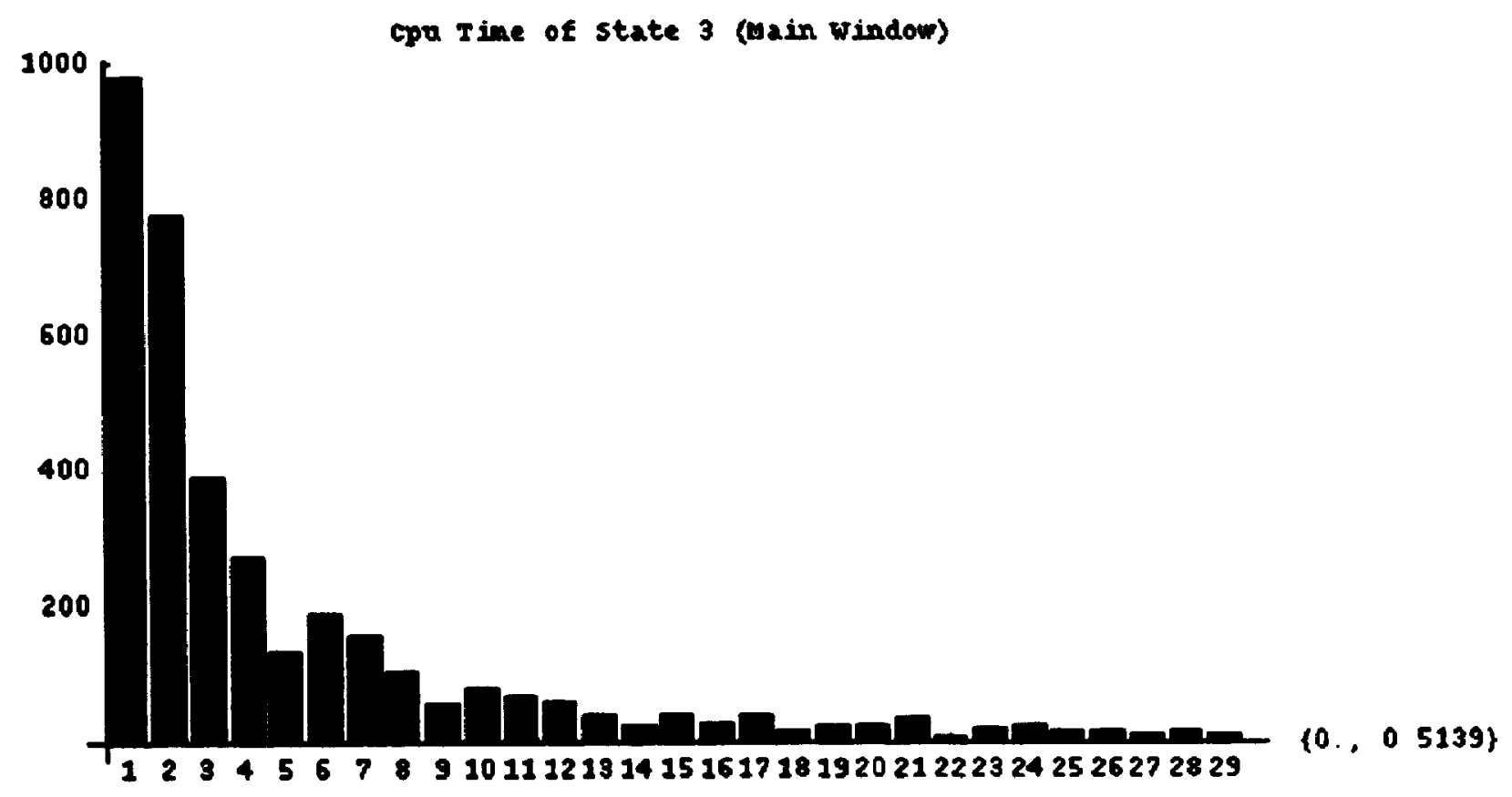

Figure B.3. Distribution of cpu time of "Main Window" state. 29 equal cells in the range $(0,0.5139)$ seconds 
cpo rine of state 5 (open ver)

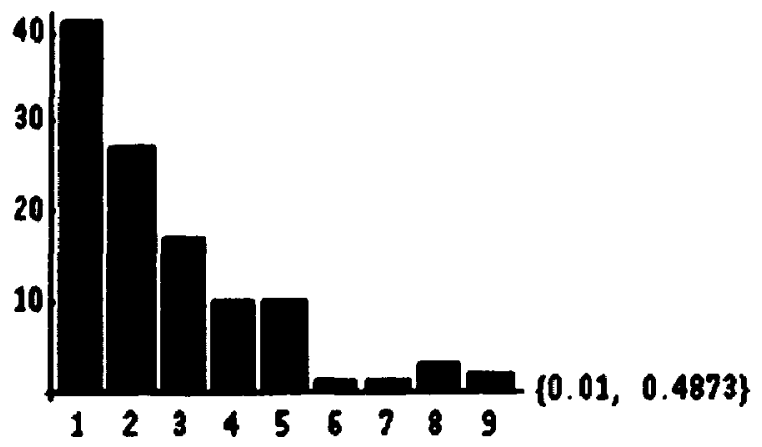

Figure B.5. Distribution of cpu time of "Open URL" state, 9 equal cells over $(0.01,0.4878)$ seconds

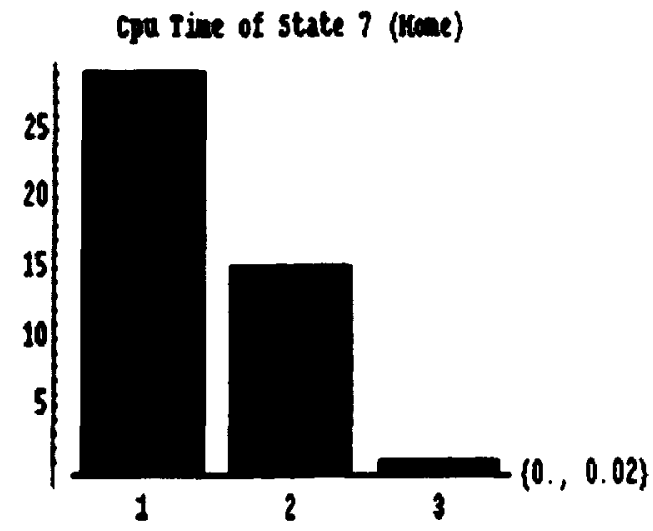

Figure B.7. Distribution of cpu time of "Home" state, 3 equal cells over $(0,0.02)$ seconds

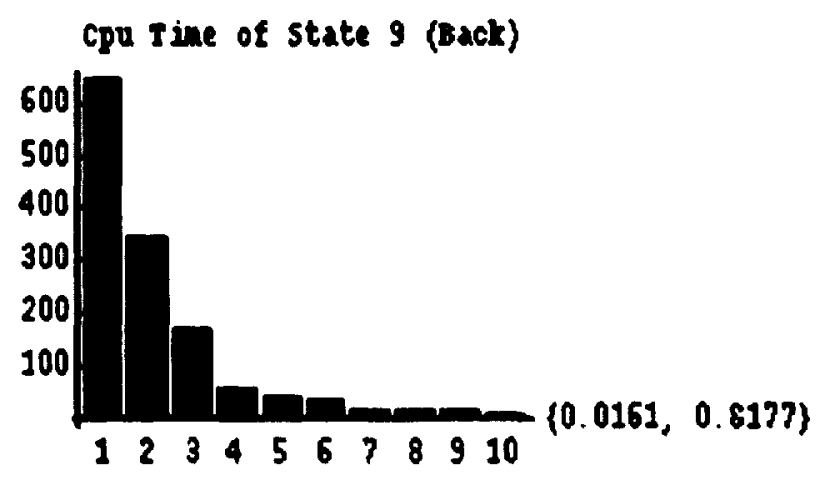

Figure B.9. Distribution of cpu time of "Back" state, 10 equal cells over $(0.0161,0.8177)$ seconds

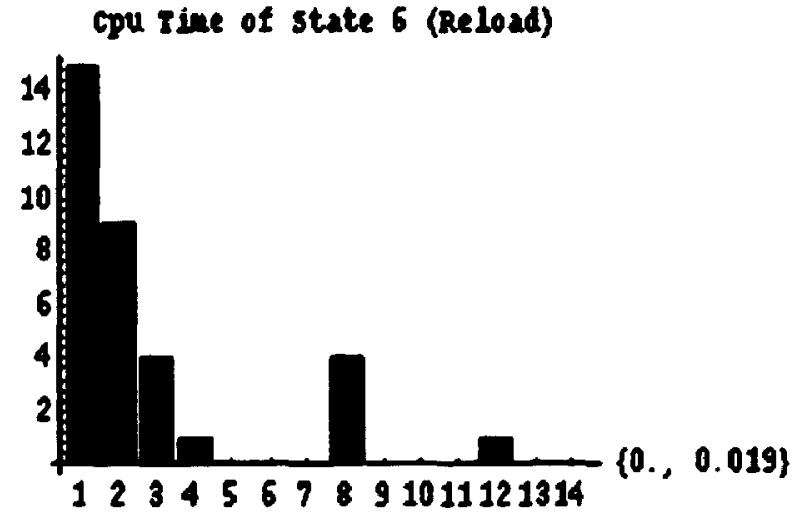

Figure B.6. Distribution of cpu time of "Reload" state. 14 equal cells over $(0,0.019)$ seconds

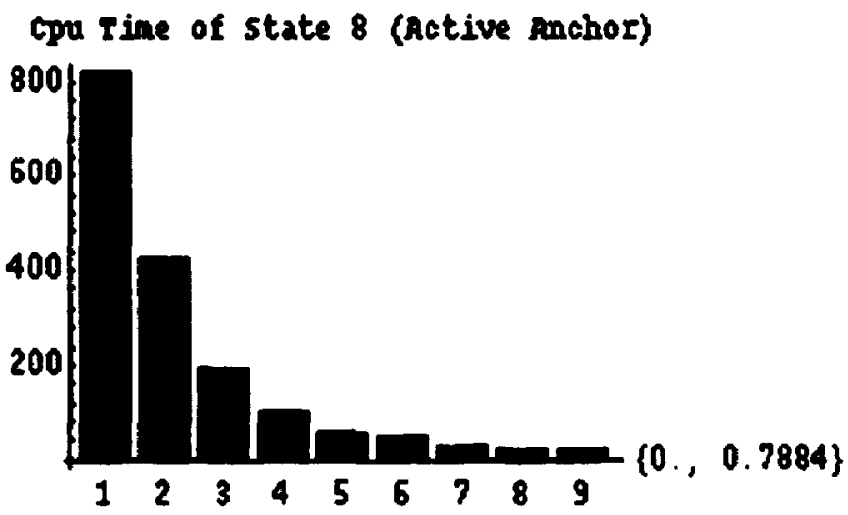

Figure B.8 Distribution of cpu time of "Active Anchor" state, 9 equal cells over $(0,0.7884)$ seconds

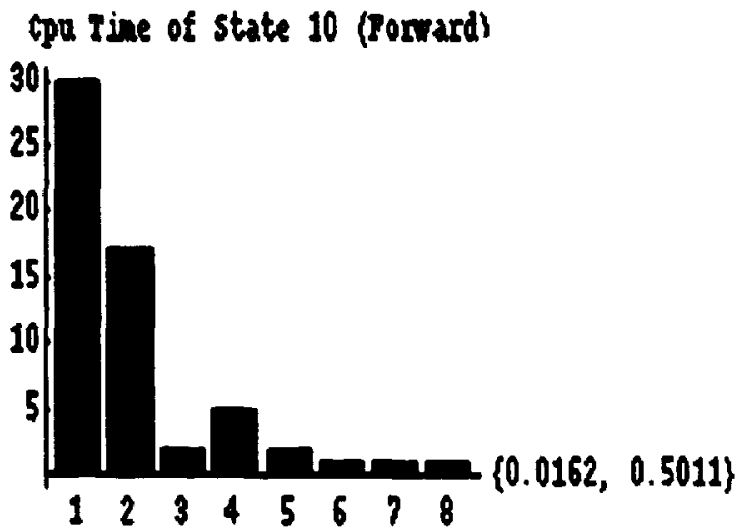

Figure B.10. Distribution of cpu time of "Forward" state, 8 equal cells over $(0.0162,0.5011)$ seconds. 


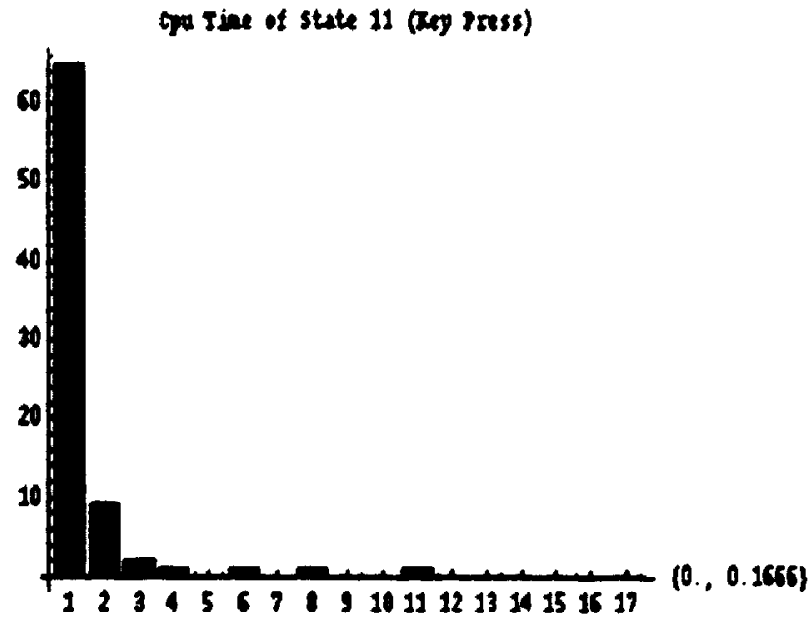

Figure B.11. Distribution of cpu time of "Key Press" state, 17 equal cells over $(0,0.1666)$ seconds

Cpu Iine of State 13 (add Motlist)

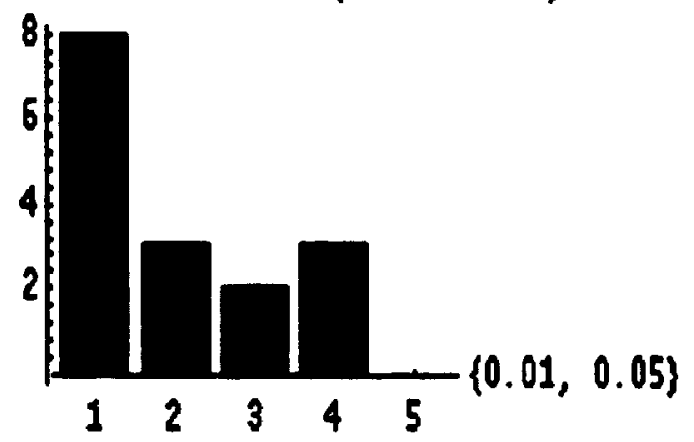

Figure B.13. Distribution of cpu time "Add Hotlist" state, 5 equal cells over $(0.01,0.05)$ seconds

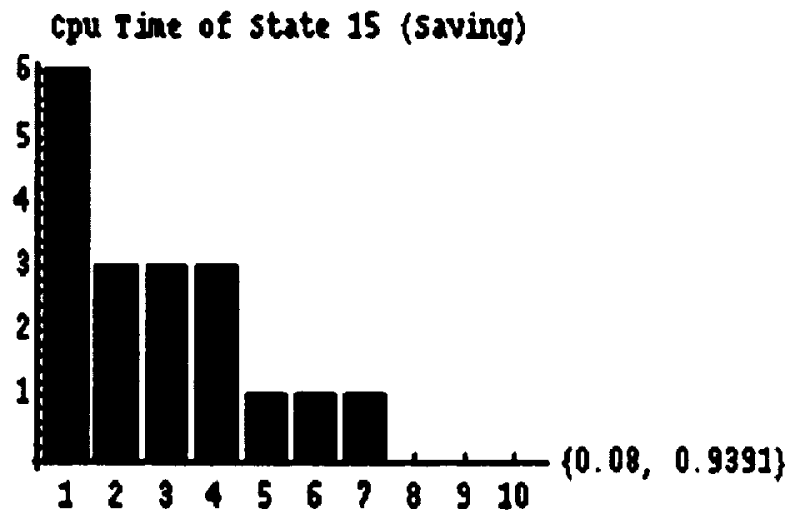

Figure B.15. Distribution of cpu time of "Saving" state, 10 equal cells over $(0.08,0.9397)$ seconds
Cpu tine of state 12 (Motlist)

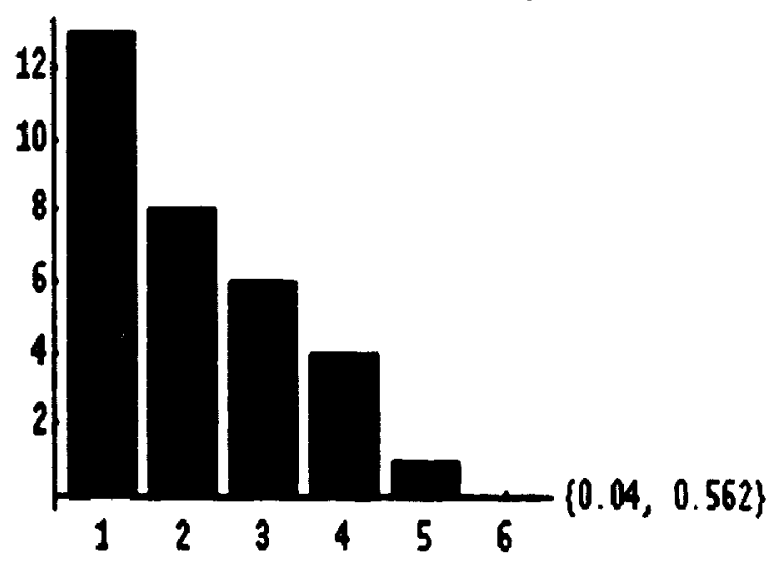

Figure B.12. Distribution of cpu time of "Hotlist" state, 6 equal cells over $(0.04,0.562)$ seconds.

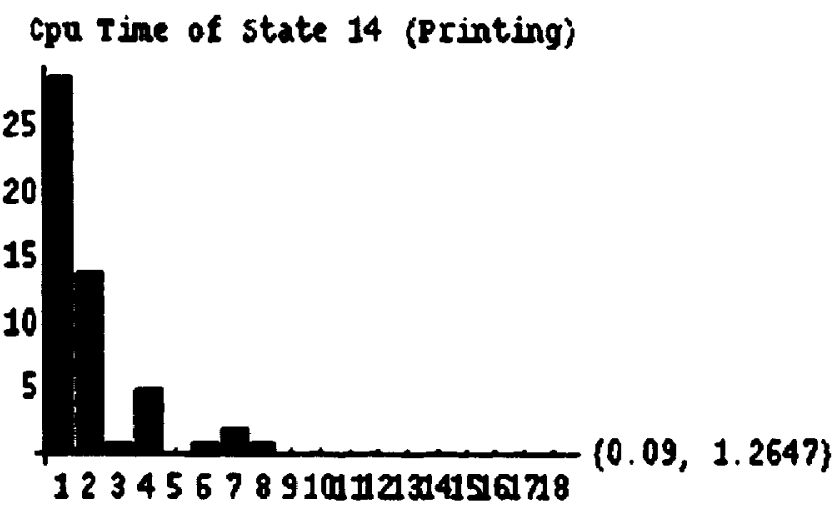

Figure B.14. Distribution of cpu time of "Printing" state, 18 equal cells over $(0.09,1.2647)$ seconds

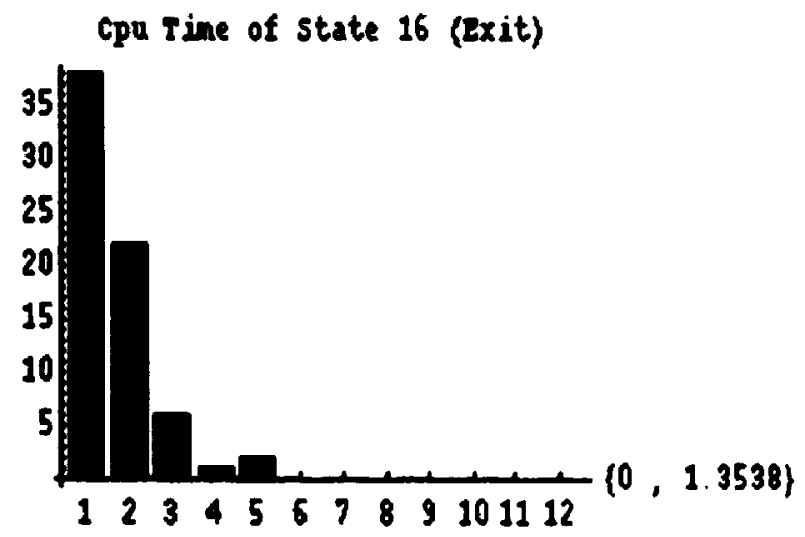

Figure B.16. Distribution of cpu time of "Exit" state, 12 equal cells over $(0,1.3538)$ seconds 


\section{Appendix C}

\section{State Parameter of Mosaic Model}

Appendix $C$ includes the parameters of the states of Mosaic Model in Figure 4.1. The numbers of the first column are parameter numbers as defined below (see 5.3):

$\begin{array}{ll}\text { Number } & \text { Parameter } \\ 1 & \text { elapsed_time } \\ 2 & \text { ru_utime } \\ 3 & \text { ru_stime } \\ 4 & \text { ru_maxrss } \\ 5 & \text { ru_idrss } \\ 6 & \text { ru_minft } \\ 7 & \text { ru_majflt } \\ 8 & \text { ru_nswap } \\ 9 & \text { u_inblock } \\ 10 & \text { ru_oublock } \\ 11 & \text { ru_msgsnd } \\ 12 & \text { ru_msgrcv } \\ 13 & \text { ru_nsignals } \\ 14 & \text { ru_nvcsw } \\ 15 & \text { ru_nivcsw } \\ 16 & \text { Bytes_loaded } \\ 17 & \text { lineCount } \\ 18 & \text { anchorCount }\end{array}$


parameter

1 elapsed_time
2 ru_utine
3 ru_stime
4 ru_maxrss
5 ru_idrss
6 ru_minflt
7 ru_majflt
8 ru_nswap
9 u_inblock
10 ru_oublock
11 ru_msgsnd
12 ru_msgrcv
13 ru_nsignals
14 ru_nvcsw
15 ru_nivcsw

Hean SD

$20.67 \quad 14.2138$

1.4963

0.6214

580.2907

31413. 151!

289.9884

178. 9419

0 .

12.9535

0.9651

227. 7093

405.093

0 .

407.1744

347.4535
0.3993

0.217

113. 0112

32264.5767

125. 7862

85.0985

0.

9. 1779

0.1846

60.6942

80.9393

0 .

111.4758

127. 2266
95* CI

(17.6225, 23.7174\}

$\{1.4107,1.5819\}$

$\{0.5749,0.6679\}$

$\{556.0611,604.5203\}$

$\{84495.6118,98330.6905\}$

\{263.0197, 316.957\}

$\{160.6967,197.187\}$

$\{0 ., 0$.

$\{10.9858,14.9212\}$

$\{0.9255,1.0047\}$

$\{214.6964,240.722 \varepsilon\}$

$\{387.7396,422.4464\}$

\{0., 0.\}

$\{383.274,431.0749\}$

$\{320.1761,374.7309\}$

Table C.1 Parameters of State 1 (Start) in Mosaic Model

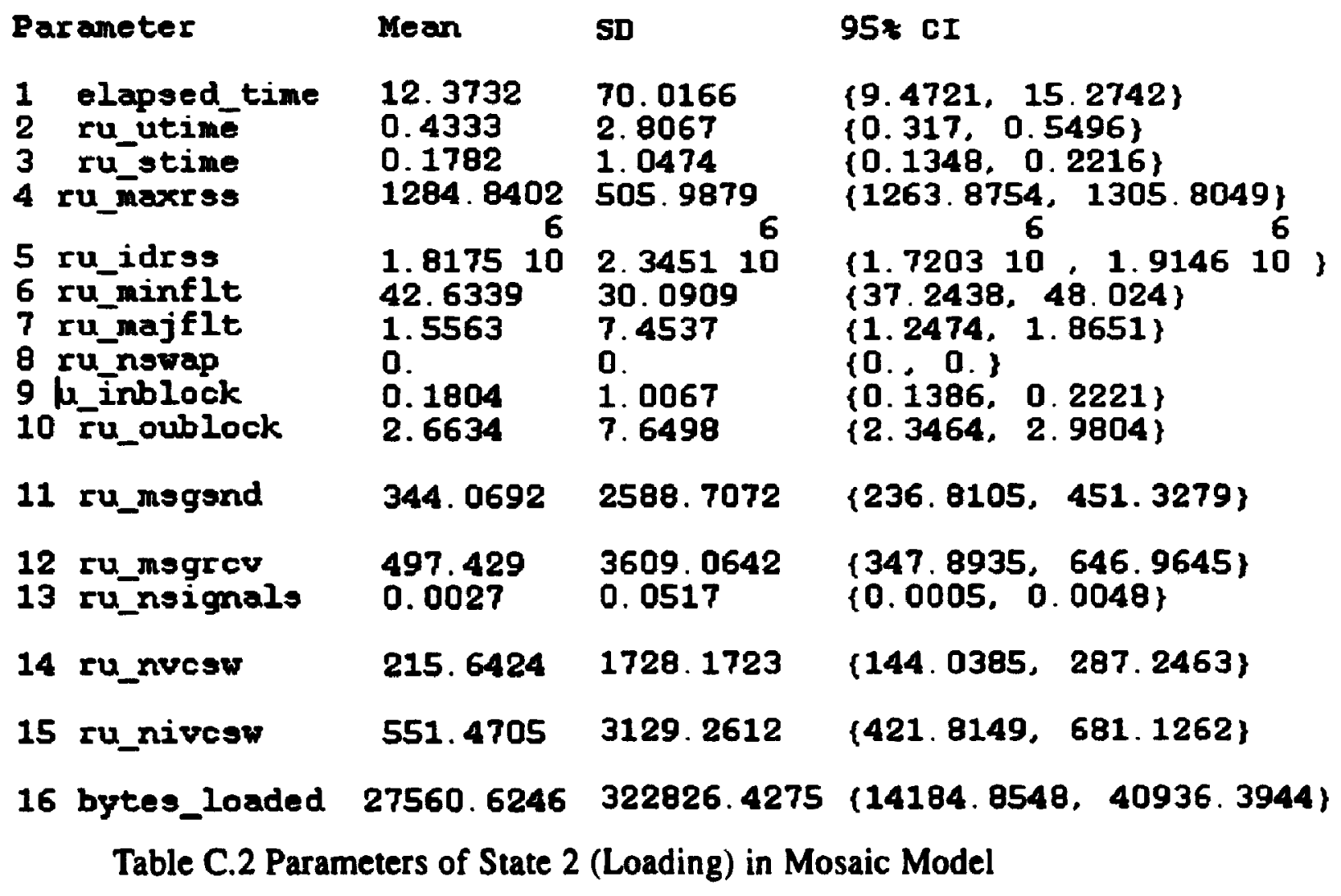




\begin{tabular}{|c|c|c|c|}
\hline Paraneter & Mean & SD & $95 * \mathrm{CI}$ \\
\hline $\begin{array}{l}1 \text { elapsed_time } \\
2 \text { ru_utine } \\
3 \text { ru_stine } \\
4 \text { ru_maxrss }\end{array}$ & $\begin{array}{l}37.0152 \\
0.0999 \\
0.0338 \\
1357.518\end{array}$ & $\begin{array}{l}147.9771 \\
0.2997 \\
0.0705 \\
466.513\end{array}$ & $\begin{array}{l}\{32.3402,41.6902\} \\
\{0.0904,0.1094\} \\
\{0.0315,0.036\} \\
\{1342.7796,1372.2565\}\end{array}$ \\
\hline $\begin{array}{l}5 \text { ru_idrss } \\
6 \text { ru_minflt } \\
7 \text { ru_majflt } \\
8 \text { ru_nswap } \\
9 \text { u_inblock } \\
10 \text { ru_oublock } \\
11 \text { ru_msgsnd } \\
12 \text { ru_msgrco } \\
13 \text { ru_nsignals } \\
14 \text { ru_nvcsw } \\
15 \text { ru_nivcsw } \\
17 \text { linecount } \\
18 \text { anchorCount }\end{array}$ & $\begin{array}{l}1.996710 \\
33.6092 \\
0.9525 \\
0 . \\
0.0099 \\
0.1838 \\
42.1161 \\
137.1288 \\
0 . \\
129.677 \\
33.0989 \\
88.8972 \\
31.336\end{array}$ & $\begin{array}{l}2.345110 \\
110.8486 \\
12.7434 \\
0 . \\
0.1609 \\
3.4605 \\
91.6131 \\
242.0182 \\
0 . \\
205.5729 \\
2090512 \\
230.544 \\
82.4732\end{array}$ & $\begin{array}{l}\{1.922610,2.070810\} \\
\{30.1072,37.1112\} \\
\{0.5499,1.3551\} \\
\{0.0 .\} \\
\{0.0048,0.0149\} \\
\{0.0745,0.2932\} \\
\{39.2218,45.0104\} \\
\{129.4828,144.7748\} \\
\{0.0 .\} \\
\{123.1823,136.1716\} \\
\{26.4944,39.7035\} \\
\{81.6136,96.1807\} \\
\{28.7305,33.9416\}\end{array}$ \\
\hline
\end{tabular}

Table C.3 Parameters of State 3 (Main Window) in Mosaic Model

\begin{tabular}{|c|c|c|c|}
\hline Paraneter & Kean & SD & 95* CI \\
\hline $\begin{array}{l}1 \text { elapsed_time } \\
2 \text { ru_utine } \\
3 \text { ru_stine } \\
4 \text { ru_naxrss }\end{array}$ & $\begin{array}{l}0.0065 \\
0.004 \\
0.0011 \\
1630.7639\end{array}$ & $\begin{array}{l}0.0058 \\
0.0049 \\
0.0032 \\
459.8415\end{array}$ & $\begin{array}{l}\{0.0051,0.0079\} \\
\{0.0029,0.0052\} \\
\{0.0004,0.0019\} \\
\{1522.7064,1738.8213\}\end{array}$ \\
\hline $\begin{array}{l}5 \text { ru_idrss } \\
6 \text { ru_minflt } \\
7 \text { ru_majflt } \\
8 \text { ru_nswap } \\
9 \text { u_inblock } \\
10 \text { ru_oublock } \\
11 \text { ru_msgsnd } \\
12 \text { ru_msgrcy } \\
13 \text { ru_nsignals } \\
14 \text { ru_nvcsw } \\
15 \text { ru_nivesw }\end{array}$ & $\begin{array}{l}2.974510 \\
1.5833 \\
0.0278 \\
0 . \\
0 . \\
0.0833 \\
1 . \\
0 . \\
0 . \\
0.0694 \\
0.2917\end{array}$ & $\begin{array}{l}\text { 2. } 989710 \\
6.3039 \\
0.1655 \\
0 . \\
0 . \\
0.2783 \\
0 . \\
0 . \\
0 . \\
0.256 \\
0.7207\end{array}$ & $\begin{array}{l}\{2.27210,3.677110\} \\
\{0.102,3.0647\} \\
\{-0.0111,0.0667\} \\
\{0 ., 0 .\} \\
\{0 ., 0 .\} \\
\{0.0179,0.1487\} \\
\{1 ., 1 .\} \\
\{0 ., 0 .\} \\
\{0.0 .\} \\
\{0.0093,0.1296\} \\
\{0.1223,0.461\}\end{array}$ \\
\hline
\end{tabular}

Table C.4 Parameters of State 4 (Submit Form) in Mosaic Model 


\section{Paraneter}

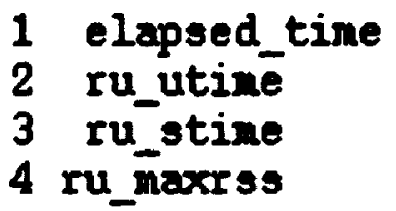

5 ru idrss

6 ru minflt

7 ru_najflt

8 ru_nstrap

9 u ínblock

10 ru_oublock

11 ru msgond

12 ru_msgrev

13 ru_nsignals

14 ru_nocsw

15 ru_nivcsw
Yean SD

38.8164

0.1125

0.062

1109. 0508

69. 6854

0. 1812

0.0849

422.0354

$845165.9492 \quad 1.399310$ 42.6864

86.4228

2. 2797

0 .

0.0339

9. 325

0.

0. 2239

0.0508

0. 2565

91.7203

174.5169

0.

204. 8814

56.5678
81.9788

176.508

0 .

186.0239

63.4747
95* CI

\{26.1117, 51.5211\}

$\{0.0794,0.1455\}$

$\{0.0465,0.0775\}$

\{1032.1075, 1185. 9942\}

6 (1032.1075, 6

\{590044.9234, 1.1003 10\}

\{26.9303, 58.4426\}

$\{0.5796,3.9797\}$

$\{0 ., 0$.

$\{-0.0069,0.0747\}$

$\{0.0041,0.0976\}$

\{76. 7744, 106.6663\}

\{142. 3369, 206.697\}

\{0., 0.\}

$\{170.9664,238.7963\}$

$\{44.9954,68.1402\}$

Table C.5 Parameters of State 5 (Open Url) in Mosaic Model

Paraneter

1 elapsed_tine
2 ru_utime
3 ru_stime
4 ru maxrss

5 ru idrss

6 ru_minflt

7 ru_majelt

8 ru_nswap

9 u_inblock

10 ru_oublock

11 ru_msgend

12 ru_msgrev

13 ru_nsignals

14 ru_nucsw

15 ru_nivesw
Mean

0.0202

0.002

0 .

1144.8

675238.2

0 .

0.2

0 .

0.2

0.2

1.

0 .

0.

0.2

0.4
SD

0.0401

0.0045

0 .

451.413

786561.6664

0 .

0.4472

0 .

0.4472

0.4472

0 .

0 .

0.

0.4472

0.8944
95* CI

$\{-0.0295,0.07\}$

$\{-0.0036,0.0076\}$

$\{0 ., 0$.

\{584.2967, 1705.3033\}

6

$-301407.1035,1.651910\}$

\{0., 0. $\}$

$\{-0.3553,0.7553\}$

$\{0 ., 0$.

$\{-0.3553,0.7553\}$

$\{-0.3553,0.7553\}$

$\{1 ., 1$.

$\{0 ., 0$.

$\{0 ., 0$.

$\{-0.3553,0.7553\}$

$\{-0.7106,1.5106\}$

Table C.6 Parameters of State 6 (Reload) in Mosaic Model 


\begin{tabular}{|c|c|c|c|}
\hline araneter & Mean & SD & 95* CI \\
\hline $\begin{array}{l}\text { elapsed_time } \\
\text { ru_utime }\end{array}$ & $\begin{array}{l}0.0191 \\
0.0029\end{array}$ & $\begin{array}{l}0.0523 \\
0.0046\end{array}$ & $\begin{array}{ll}\{0.0034, & 0.0348\} \\
\{0.0016, & 0.0043\}\end{array}$ \\
\hline $\begin{array}{l}\text { ru_stime } \\
\text { ru_maxrss }\end{array}$ & $\begin{array}{l}0.0009 \\
1151.8222 \\
6\end{array}$ & $\begin{array}{l}0.0029 \\
479.8771 \\
6\end{array}$ & $\begin{array}{l}\{0 ., 0.0018\} \\
\{1007.6512,1295.9933\}\end{array}$ \\
\hline $\begin{array}{l}\text { ru_idrss } \\
\text { ru_minflt } \\
\text { ru_majflt } \\
\text { ru_nswap } \\
\text { u_inblock } \\
0 \text { ru_oublock } \\
1 \text { ru_msgsnd } \\
2 \text { ru_msgrce } \\
3 \text { ru_nsignals } \\
4 \text { ru_nucsw } \\
5 \text { ru_nivcsw }\end{array}$ & $\begin{array}{l}1.555810 \\
7.8222 \\
0.7333 \\
0 . \\
0.0222 \\
0.0222 \\
0.9778 \\
0 . \\
0 . \\
0.8 \\
0.2444\end{array}$ & $\begin{array}{l}2.432610 \\
15.2529 \\
2.9802 \\
0 . \\
0.1491 \\
0.1491 \\
0.1491 \\
0 . \\
0 . \\
2.9741 \\
0.6794\end{array}$ & $\begin{array}{l}\{824982.3854,2.286710\} \\
\{3.2398,12.4047\} \\
\{-0.162,1.6287\} \\
\{0.0 .\} \\
\{-0.0226,0.067\} \\
\{-0.0226,0.067\} \\
\{0.933,1.0226\} \\
\{0.0 .\} \\
\{0.0 .\} \\
\{-0.0935,1.6935\} \\
\{0.0403,0.4486\}\end{array}$ \\
\hline
\end{tabular}

Table C.7 Parameters of State 7 (Home) in Mosaic Model

$\begin{array}{llll}\text { Paraneter } & \text { Mean } & \text { SD } & 95 * \text { CI } \\ 1 \text { elapsed_time } & 0.5136 & 0.8245 & \{0.4753,0.5519\} \\ 2 \text { ru_utime } & 0.1983 & 0.4536 & \{0.1773,0.2194\} \\ 3 \text { ru_stime } & 0.0215 & 0.0266 & \{0.0203,0.0227\} \\ 4 \text { ru_maxrss } & 79.0717 & 317.8483 & \{64.3208,93.8226\} \\ 5 \text { ru_idrss } & 104151.453 & 673474.8361 & \{72896.3865,135406.5194\} \\ 6 \text { ru_minflt } & 20.8539 & 37.3279 & \{19.1215,22.5862\} \\ 7 \text { ru_majflt } & 0.4507 & 3.0991 & \{0.3069,0.5946\} \\ 8 \text { ru_nswap } & 0 . & 0 . & \{0.0 .\} \\ 9 \text { u_inblock } & 0.0213 & 0.1482 & \{0.0144,0.0282\} \\ 10 \text { ru_oublock } & 0.0997 & 0.3107 & \{0.0852,0.1141\} \\ 11 \text { ru_msgsnd } & 29.0997 & 43.5328 & \{27.0794,31.12\} \\ 12 \text { ru_msgrce } & 27.5134 & 37.2174 & \{25.7862,29.2406\} \\ 13 \text { ru_nsignals } & 0.0437 & 1.8457 & \{-0.042,0.1293\} \\ 14 \text { ru_nvcsw } & 80.6667 & 60.9816 & \{64.0219,97.3114\} \\ 15 \text { ru_nivcsw } & 20.3807 & 34.9197 & \{18.7602,22.0013\}\end{array}$

Table C. 8 Parameters of utate 8 (Active Anchor) in Mosaic Model 
Parameter

1 elapsed_tine
2 ru_utine
3 ru_stime
4 ru_maxrss
5 ru_idrss
6 ru_minflt
7 ru_majflt
8 ru_nswap
9 u_inblock
10 ru_oublock
11 ru_msgsnd
12 ru_msgrcv

13 ru_nsignals

14 ru_nvcsw

15 ru_nivesw
Mean

0.4149

0. 1498

0.0192

1329. 4871

$2.1967 \quad \begin{array}{r}6 \\ 10\end{array}$

27. 8554

0.3257

0.

T. 6385

0.0198

21. 9559

15. 9513

1053. 6111

9.4033

13. 1697
SD

0.5324

0.2375

0.0245

577.6424

6

2.408410

65.3205

1. 7933

0 .

276.5029

1. 1811

218.5495

472. 0913

38192.4945

367.2424

19. 0258
95* CI

$\{0.3861,0.4437\}$

$\{0.1369,0.1626\}$

$\{0.0179,0.0205\}$

\{1298.2258, 1360.7484\} 6

$\{2.066310,2.32710\}$

(24. 3203, 31. 3905)

$\{0.2287,0.4228\}$

\{0., 0. \}

$\{-? .3255,22.6025\}$

$\{-0.0441,0.0837\}$

$\{10.1282,33.7835\}$

$\{-9.5977,41.5003\}$

$\{-1013.3205,3120.5428\}$

$\{-10.4714,29.2781\}$

$\{12.1401,14.1994\}$

Table C.9 Parameters of State 9 (Back) in Mosaic Model

\begin{tabular}{|c|c|c|c|}
\hline Paraneter & Mean & SD & $95 \% \mathrm{CI}$ \\
\hline $\begin{array}{l}1 \text { elapsed_time } \\
2 \text { ru_utime } \\
3 \text { ru_stime } \\
4 \text { ru_maxrss }\end{array}$ & $\begin{array}{l}0.3361 \\
0.16 \\
0.0121 \\
1365.0317 \\
\end{array}$ & $\begin{array}{l}0.3841 \\
0.2336 \\
0.0161 \\
436.2526 \\
6\end{array}$ & $\begin{array}{l}\{0.2394,0.4328\} \\
\{0.1012,0.2189\} \\
\{0.0081,0.0162\} \\
\{1255.163,1474.9005\} \\
6{ }_{6}{ }^{2},\end{array}$ \\
\hline $\begin{array}{l}5 \text { ru_idrss } \\
6 \text { ru_minflt } \\
7 \text { ru_majflt } \\
8 \text { ru_nswap } \\
9 \text { u_inblock } \\
10 \text { ru_oublock } \\
11 \text { ru_msgsnd } \\
12 \text { ru_msgrcy } \\
13 \text { ru_nsignals } \\
14 \text { ru_nvcsw } \\
15 \text { ru_nivcsw }\end{array}$ & $\begin{array}{l}2.045210 \\
12.0159 \\
0.2698 \\
0 . \\
0.0317 \\
0.0952 \\
24.0952 \\
22.127 \\
0 . \\
12.9365 \\
14.9841\end{array}$ & $\begin{array}{l}2.19310 \\
46.1182 \\
2.1418 \\
0 . \\
0.252 \\
0.2959 \\
36.7488 \\
20.9049 \\
0 . \\
23.911 \\
24.2424\end{array}$ & $\begin{array}{l}\{1.492910,2.597510 \\
\{0.4012,23.6306\} \\
\{-0.2696,0.8092\} \\
\{0.0 .\} \\
\{-0.0317,0.0952\} \\
\{0.0207,0.1698\} \\
\{14.8402,33.3503\} \\
\{16.8621,27.3918\} \\
\{0.0 .\} \\
\{6.9146,18.9584\} \\
\{8.8788,21.0895\}\end{array}$ \\
\hline
\end{tabular}

Table C.10 Parameters of State 10 (Forward) in Mosaic Model 


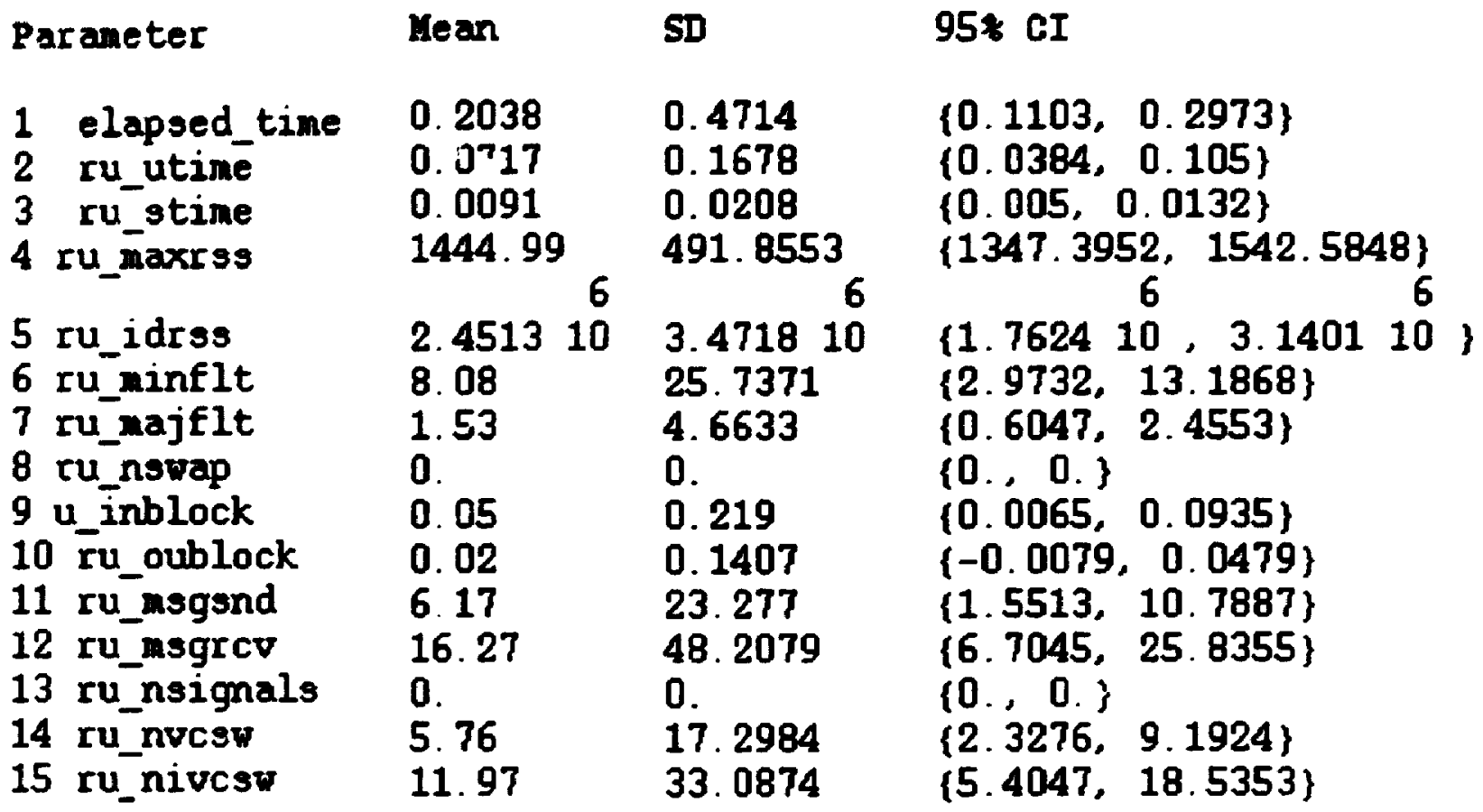

Table C.11 Parameters of State 11 (Key Press) in Mosaic Model

\begin{tabular}{|c|c|c|c|}
\hline Paraneter & Mean & SD & 95* CI \\
\hline $\begin{array}{l}1 \text { elapsed_time } \\
2 \text { ru_utine } \\
3 \text { ru_stine } \\
4 \text { ru_maxrss }\end{array}$ & $\begin{array}{l}25.4717 \\
0.2412 \\
0.0585 \\
610.2059\end{array}$ & $\begin{array}{l}29.5638 \\
0.4048 \\
0.0554 \\
758.6918\end{array}$ & $\begin{array}{l}\{15.1564,35.787\} \\
\{0.0999,0.3824\} \\
\{0.0392,0.0779\} \\
\{345.4858,874.926\}\end{array}$ \\
\hline $\begin{array}{l}5 \text { ru_idrss } \\
6 \text { ru_minflt } \\
7 \text { ru_majflt } \\
8 \text { ru_nswap } \\
9 \text { u_inblock } \\
10 \text { ru_oublock } \\
11 \text { ru_msgsnd } \\
12 \text { ru_msgrev } \\
13 \text { ru_nsignals } \\
14 \text { ru_nucsw } \\
15 \text { ru_nivcsw }\end{array}$ & $\begin{array}{l}1.424210 \\
80.9412 \\
6.3235 \\
0 . \\
0.0882 \\
0.5882 \\
44.2353 \\
78 . \\
0 . \\
83.1176 \\
16.6176\end{array}$ & $\begin{array}{l}2.857410 \\
88.1438 \\
14.2014 \\
0 . \\
0.3788 \\
1.0479 \\
27.5627 \\
46.4888 \\
0 . \\
48.9624 \\
15.2316\end{array}$ & $\begin{array}{l}\{427172.069,2.421210\} \\
\{50.1864,111.696\} \\
\{1.3684,11.2786\} \\
\{0.0 .\} \\
\{-0.0439,0.2204\} \\
\{0.2226,0.9539\} \\
\{34.6182,53.8524\} \\
\{61.7793,94.2207\} \\
\{0.0 .\} \\
\{66.0338,100.2014\} \\
\{11.3031,21.9322\}\end{array}$ \\
\hline
\end{tabular}

Table C.12 Parameters of State 12 (Hotlist) in Mosaic $M$ del 
Mean SD

95* CI

\begin{tabular}{|c|c|c|c|}
\hline $\begin{array}{l}1 \text { elapsed_time } \\
2 \text { ru_utine } \\
3 \text { ru_stine } \\
4 \text { ru_maxrss }\end{array}$ & $\begin{array}{l}0.163 \\
0.0094 \\
0.0181 \\
1589.25\end{array}$ & $\begin{array}{l}0.1015 \\
0.0077 \\
0.0138 \\
358.6507\end{array}$ & $\begin{array}{l}\{0.109,0.2171\} \\
\{0.0053,0.0135\} \\
\{0.0108,0.0255\} \\
\{1398.1385,1780.3615\}\end{array}$ \\
\hline $\begin{array}{l}5 \text { ru_idrss } \\
6 \text { ru_minflt } \\
7 \text { ru_majflt } \\
8 \text { ru_nswap } \\
9 \text { u_inblock } \\
10 \text { ru_oublock } \\
11 \text { ru_nsgsnd } \\
12 \text { ru_msgrco } \\
13 \text { ru_nsignals } \\
14 \text { ru_nvcsw } \\
15 \text { ru nivcsw }\end{array}$ & $\begin{array}{l}2.331610 \\
21.9375 \\
2.625 \\
0 . \\
0.875 \\
1.0625 \\
0 . \\
0 . \\
0 . \\
9.25 \\
1.125\end{array}$ & $\begin{array}{l}2.217210 \\
18.7988 \\
5.005 \\
0 . \\
1.7464 \\
0.25 \\
0 . \\
0 . \\
0 . \\
5.3229 \\
2.029\end{array}$ & $\begin{array}{l}\{1.1502,10,3.513110\} \\
\{11.9203,31.9547\} \\
\{-0.042,5.292\} \\
\{0.0 .\} \\
\{-0.0556,1.8056\} \\
\{0.9293,1.1957\} \\
\{0.0 .\} \\
\{0.0 .\} \\
\{0.0 .\} \\
\{6.4136,12.0864\} \\
\{0.0438,2.2062\}\end{array}$ \\
\hline
\end{tabular}

Table C.13 Parameters of State 13 (Add Hotlist) in Mosaic Model

\begin{tabular}{|c|c|c|c|}
\hline Paraneter & Kean & SD & $95 * C I$ \\
\hline $\begin{array}{l}1 \text { elapsed_time } \\
2 \text { ru_utime } \\
3 \text { ru_stime } \\
4 \text { ru_maxrss }\end{array}$ & $\begin{array}{l}5.8677 \\
0.0935 \\
0.1163 \\
1166.9444 \\
6\end{array}$ & $\begin{array}{l}9.7104 \\
0.1419 \\
0.0527 \\
? .50 .5724\end{array}$ & $\begin{array}{l}\{3.2173,8.5181\} \\
\{0.0548,0.1323\} \\
\{0.1019,0.1307\} \\
\{1098,5513,1235.3376\}\end{array}$ \\
\hline $\begin{array}{l}5 \text { ru_idrss } \\
6 \text { ru_minflt } \\
7 \text { ru_majflt } \\
8 \text { ru_nswap } \\
9 \text { u_inblock } \\
10 \text { ru_oublock } \\
11 \text { ru_msgsnd } \\
12 \text { ru_msgrco } \\
13 \text { ru_nsignals } \\
14 \text { ru_nocsw } \\
15 \text { ru_nivcsw }\end{array}$ & $\begin{array}{l}1.085610 \\
207.2222 \\
1.5 \\
0 . \\
0.8333 \\
13.8704 \\
26.7407 \\
52.7407 \\
0 . \\
80.6667 \\
12.7963\end{array}$ & $\begin{array}{l}766433.1515 \\
66.0233 \\
3.8936 \\
0 . \\
2.09 \\
3.5557 \\
22.5574 \\
52.2989 \\
0 . \\
60.9816 \\
18.4824\end{array}$ & $\begin{array}{l}\{876384.6311,1.294810\} \\
\{189.2013,225.2431\} \\
\{0.4372,2.5628\} \\
\{0.0 .\} \\
\{0.2629,1.4038\} \\
\{12.8998,14.8409\} \\
\{20.5838,32.8977\} \\
\{38.4659,67.0156\} \\
\{0 ., 0 .\} \\
\{64.0219,97.3114\} \\
\{7.7516,17.841\}\end{array}$ \\
\hline
\end{tabular}

Table C.14 Parameters of State 14 (Printing) in Mosaic Model 


\begin{tabular}{|c|c|c|c|}
\hline Paraneter & Lean & SD & $95 * \mathrm{CI}$ \\
\hline $\begin{array}{l}1 \text { elapsed_tine } \\
2 \text { ru_utine } \\
3 \text { ru_stime } \\
4 \text { ru_maxrss }\end{array}$ & $\begin{array}{l}24.639 \\
0.2205 \\
0.1147 \\
1377.8947\end{array}$ & $\begin{array}{l}15.6013 \\
0.1645 \\
0.1137 \\
179.2565\end{array}$ & $\begin{array}{l}\{17.1194,32.1586\} \\
\{0.1413,0.2998\} \\
\{0.0599,0.1695\} \\
\{1291.4959,1464.2936\} \\
6\end{array}$ \\
\hline $\begin{array}{l}5 \text { ru_idrss } \\
6 \text { ru_minflt } \\
7 \text { ru_majflt } \\
8 \text { ru_nswap } \\
9 \text { u_inblock } \\
10 \text { ru_oublock } \\
11 \text { ru_msgsnd } \\
12 \text { ru_msgrcv } \\
13 \text { ru_nsignals } \\
14 \text { ru_nvcsw } \\
15 \text { ru_nivcsw }\end{array}$ & $\begin{array}{l}1.503910 \\
114.0526 \\
5.7368 \\
0 . \\
0.1579 \\
1.5263 \\
66.3158 \\
133.5789 \\
0 . \\
218.7368 \\
64.2632\end{array}$ & $\begin{array}{l}1.039210 \\
89.1537 \\
9.0728 \\
0 . \\
0.5015 \\
1.6455 \\
43.4358 \\
90.4939 \\
0 . \\
154.1424 \\
94.6061\end{array}$ & $\begin{array}{l}\{1.00310,2.004810\} \\
\{71.0819,157.0233\} \\
\{1.3639,10.1098\} \\
\{0 ., 0 .\} \\
\{-0.0838,0.3996\} \\
\{0.7332,2.3194\} \\
\{45.3804,87.2512\} \\
\{89.9623,177.1956\} \\
\{0.0 .\} \\
\{144.4426,293.0311\} \\
\{18.6645,109.8618\}\end{array}$ \\
\hline
\end{tabular}

Table C.15 Parameters of State 15 (Saving) in Mosaic Model

\begin{tabular}{|c|c|c|c|}
\hline arameter & Mean & SD & 95* CI \\
\hline $\begin{array}{l}1 \text { elapsed_time } \\
2 \text { ru_utime } \\
3 \text { ru_stime } \\
4 \text { ru_maxrss }\end{array}$ & $\begin{array}{l}18.3482 \\
0.0934 \\
0.0665 \\
1402.1143 \\
6\end{array}$ & $\begin{array}{l}102.2149 \\
0.1649 \\
0.0597 \\
494.4723 \\
6\end{array}$ & $\begin{array}{l}\{-6.0241,42.7205\} \\
\{0.0541,0.1328\} \\
\{0.0523,0.0807\} \\
\{1284.2115,1520.0171\} \\
6\end{array}$ \\
\hline $\begin{array}{l}5 \text { ru_idrss } \\
6 \text { ru_minflt } \\
7 \text { ru_majflt } \\
8 \text { ru_nswap } \\
9 \text { u_inblock } \\
10 \text { ru_oublock } \\
11 \text { ru_msgsnd } \\
12 \text { ru_msgrcv } \\
13 \text { ru_nsignals } \\
14 \text { ru_nvcsw } \\
15 \text { ru_nivcsw }\end{array}$ & $\begin{array}{l}2.110910 \\
71.2286 \\
3.7143 \\
0 . \\
0.4857 \\
10.5857 \\
14.0286 \\
48.6857 \\
0 . \\
69.6571 \\
25.0571\end{array}$ & $\begin{array}{l}2.86210 \\
158.7846 \\
8.584 \\
0 . \\
1.032 \\
7.9006 \\
43.6245 \\
149.4143 \\
0 . \\
131.1461 \\
58.6384\end{array}$ & $\begin{array}{l}\{1.428510,2.793410\} \\
\{33.3677,109.0894\} \\
\{1.6675,5.7611\} \\
\{0.0 .\} \\
\{0.2396,0.7318\} \\
\{8.7019,12.4696\} \\
\{3.6267,24.4305\} \\
\{13.0591,84.3123\} \\
\{0.0 .\} \\
\{38.3865,100.9278\} \\
\{11.0753,39.039\}\end{array}$ \\
\hline
\end{tabular}

Table C.16 Parameters of State 16 (Exit) in Mosaic Model 


\section{Appendix D.}

\section{More Performance Data of WWW Model}

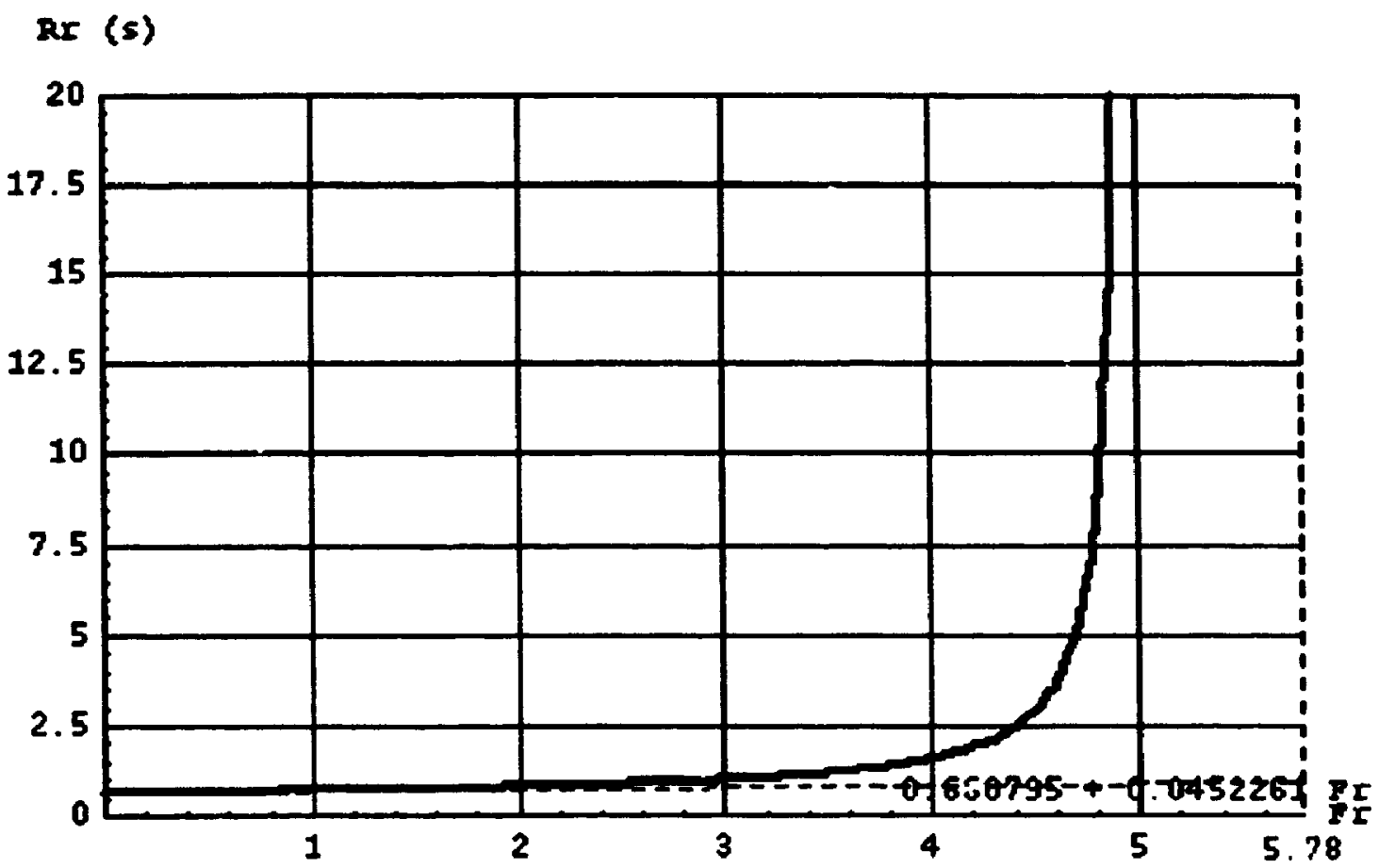

Figure D.1.1. Response Time of remote requests $R r$ vs arrival rate $F r$, with $\mathbf{5 0 0}$ local user 


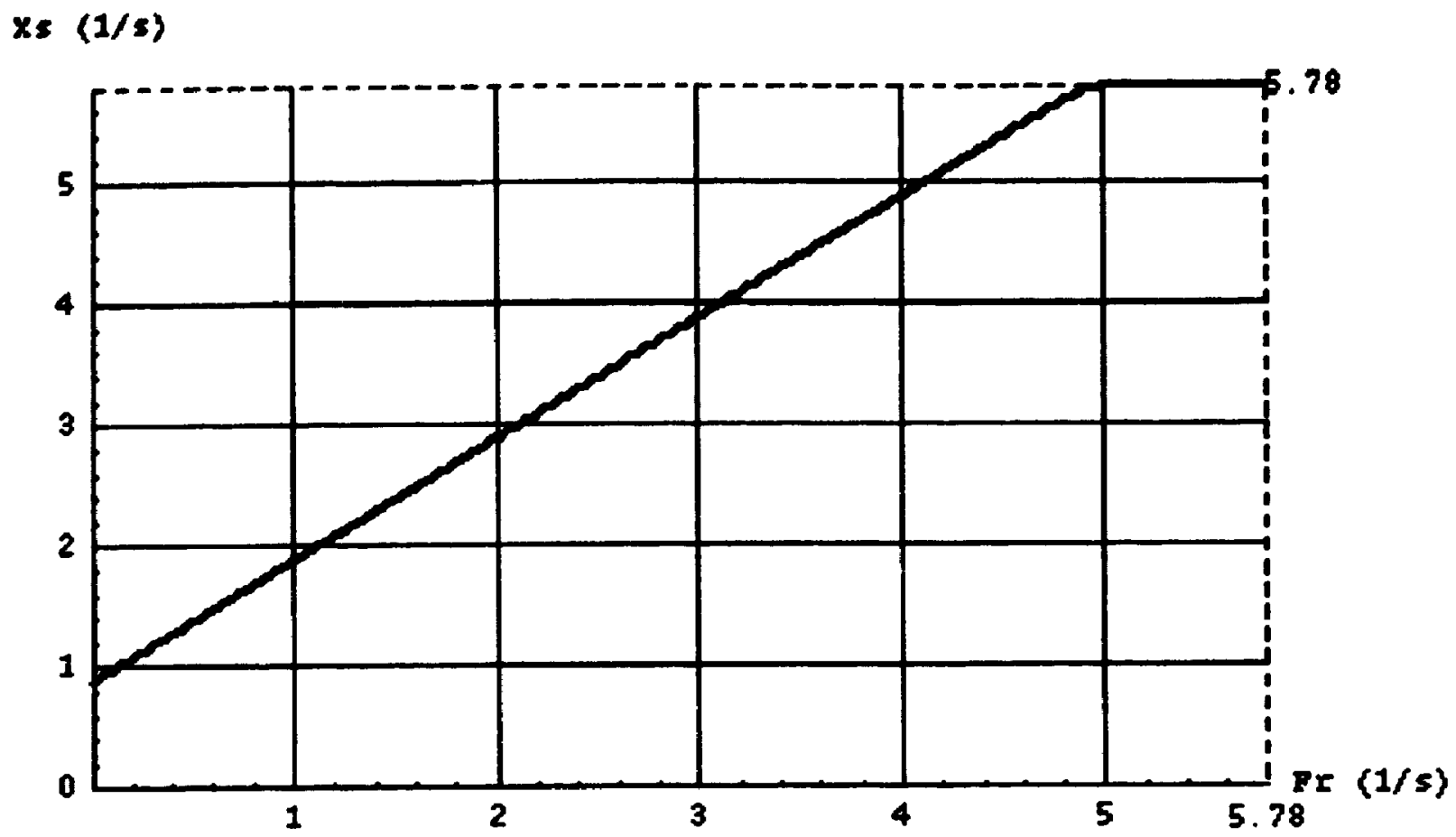

Figure D.1.2. Total request throughput Xs vs arrival rate Fr, with 500 local users

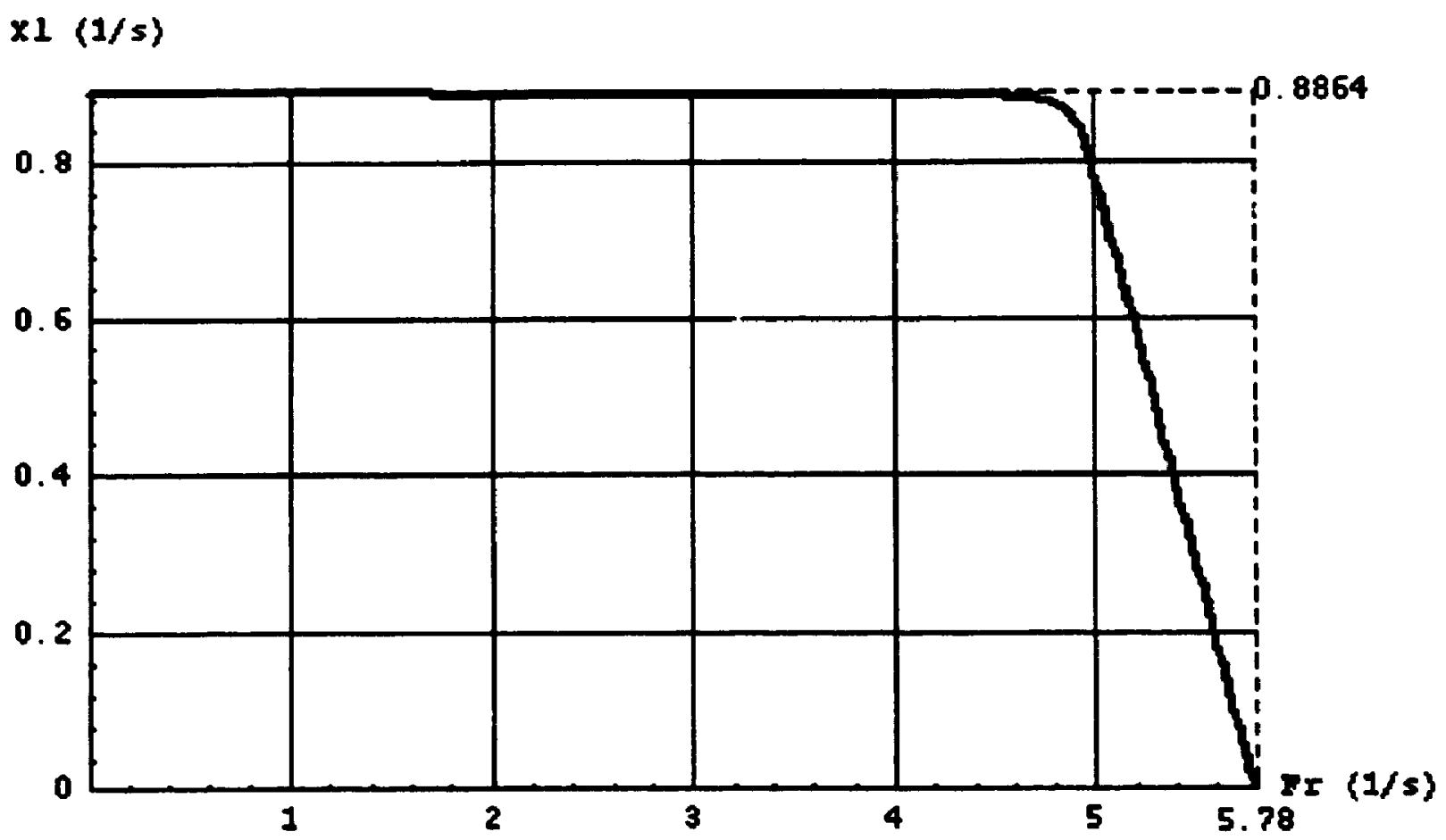

Figure D.1.3. Local request throughput $\mathrm{XI}$ vs arrival rate Fr, with 500 local users 


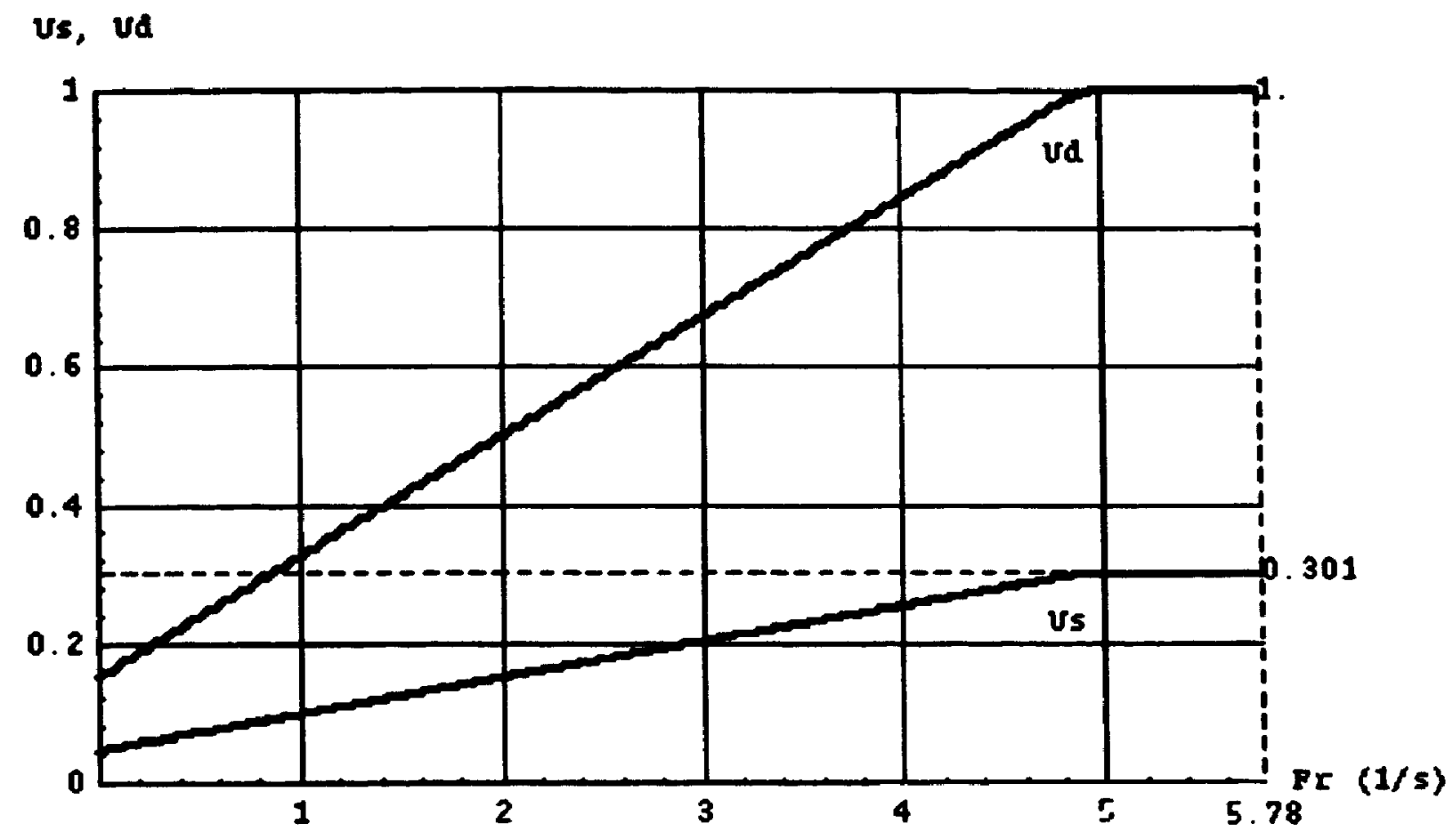

Figure D.1.4. Utilizations of the server Us and disk Ud, vs remote arrival rate $F r$, with 500 local users

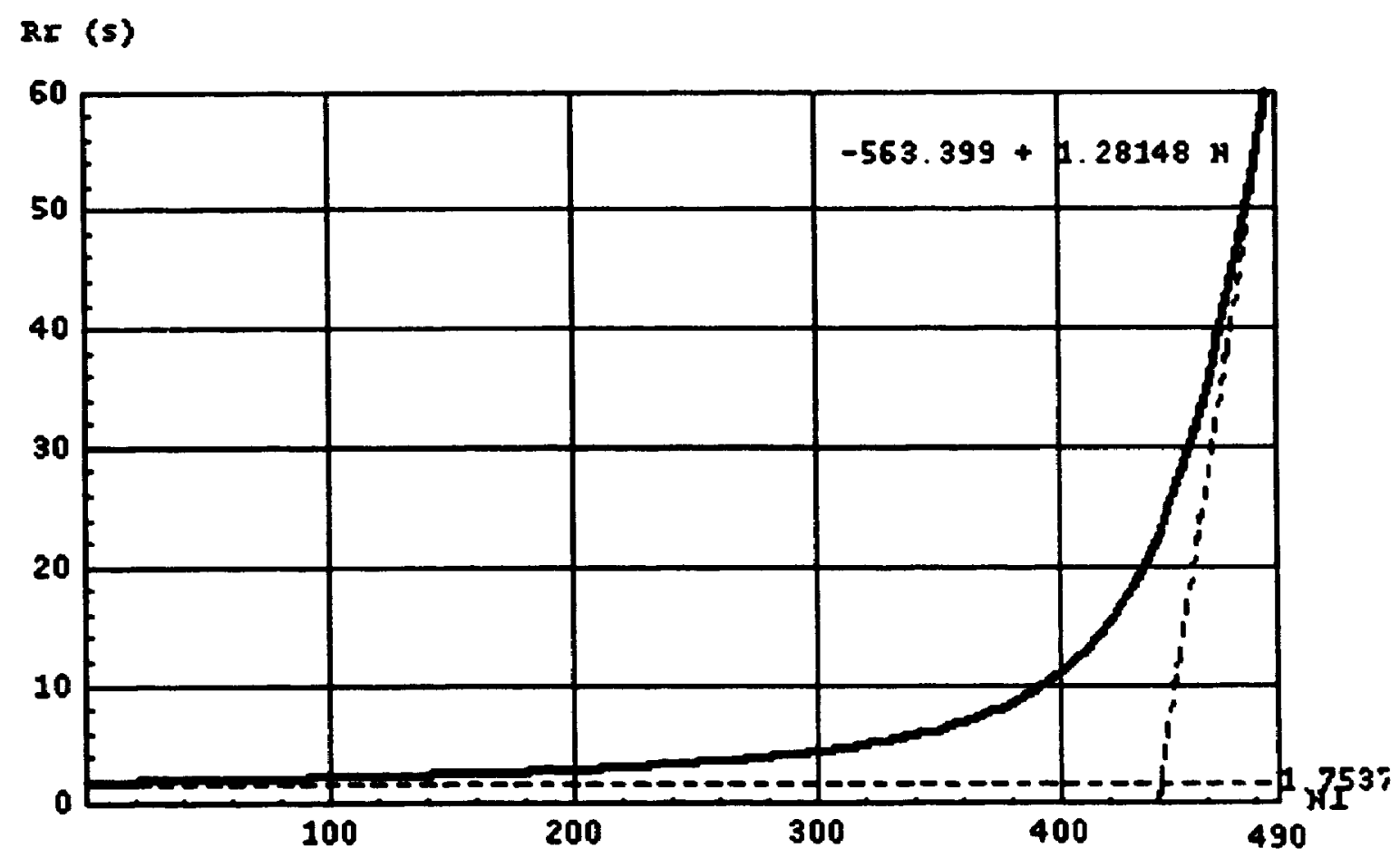

Figure D.2.1. Response time of remote requests Rr vs the number of local users NI, with remote arrival rate 5. 


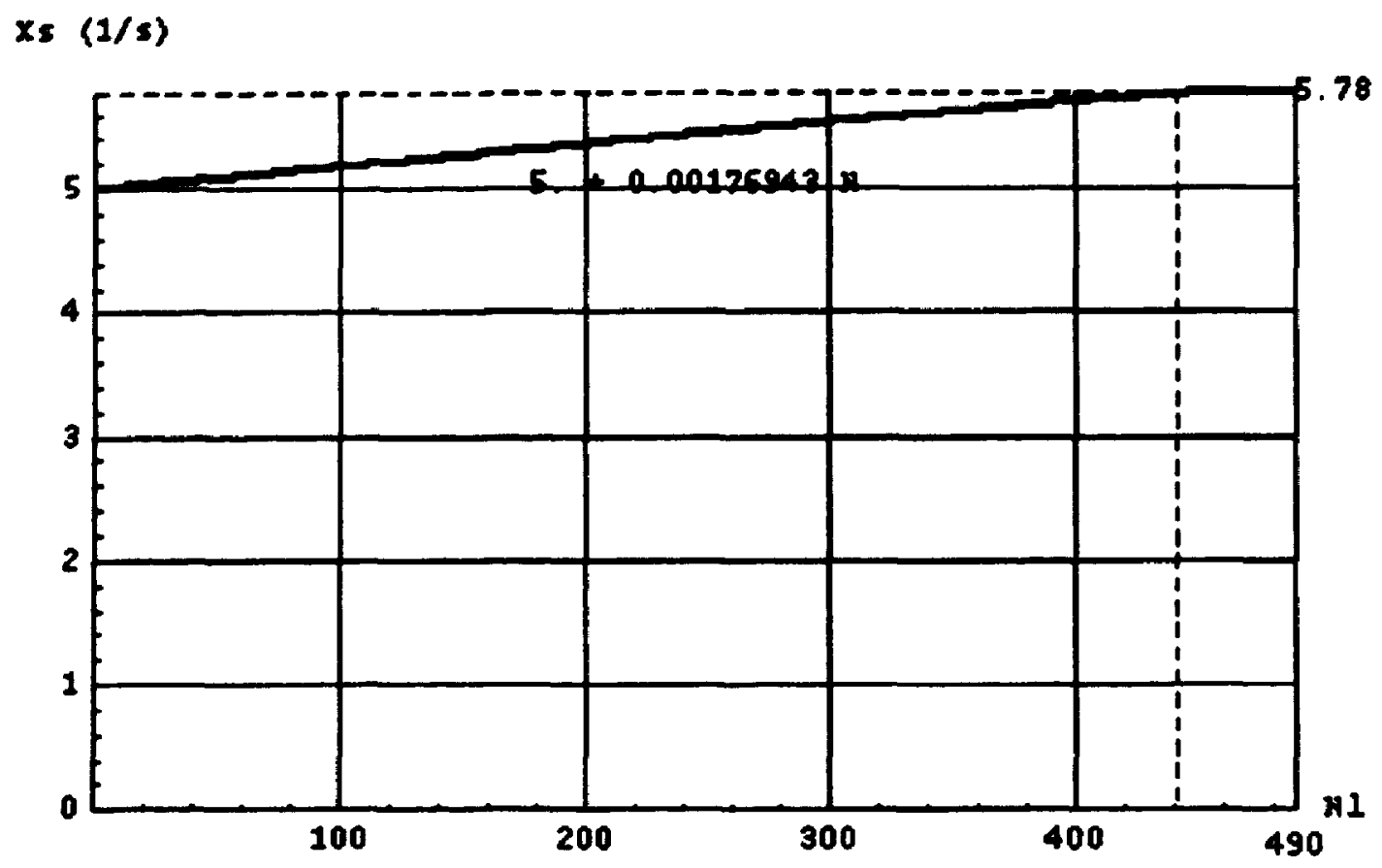

Figure D.2.2. Total request throughput Xs vs the number of local users Nl, with remote arrival rate 5 .

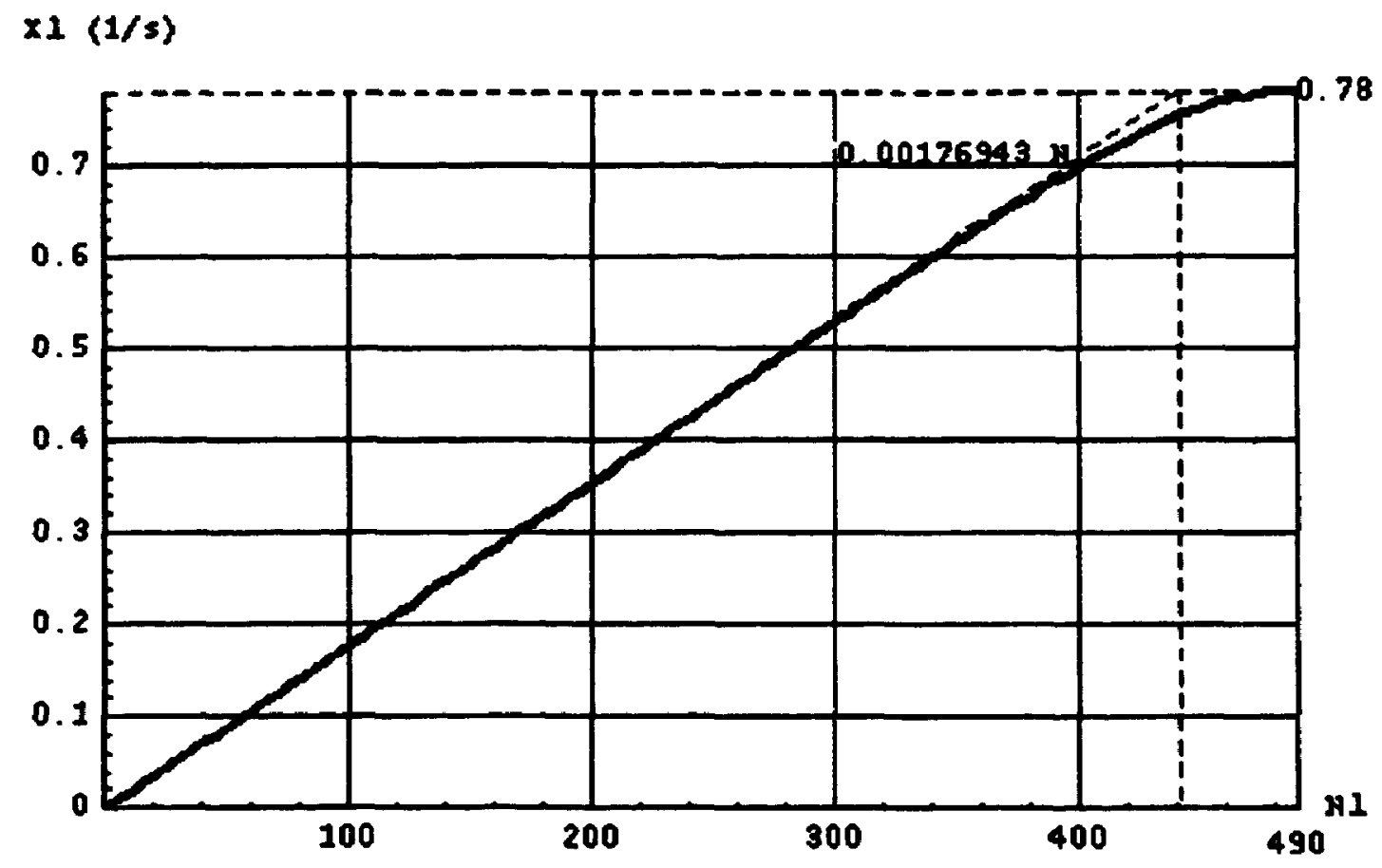

Figure D.2.3. Local request throughput $\mathrm{Xl}$ vs the number of local users $\mathrm{Nl}$, with remote arrival rate 5 . 


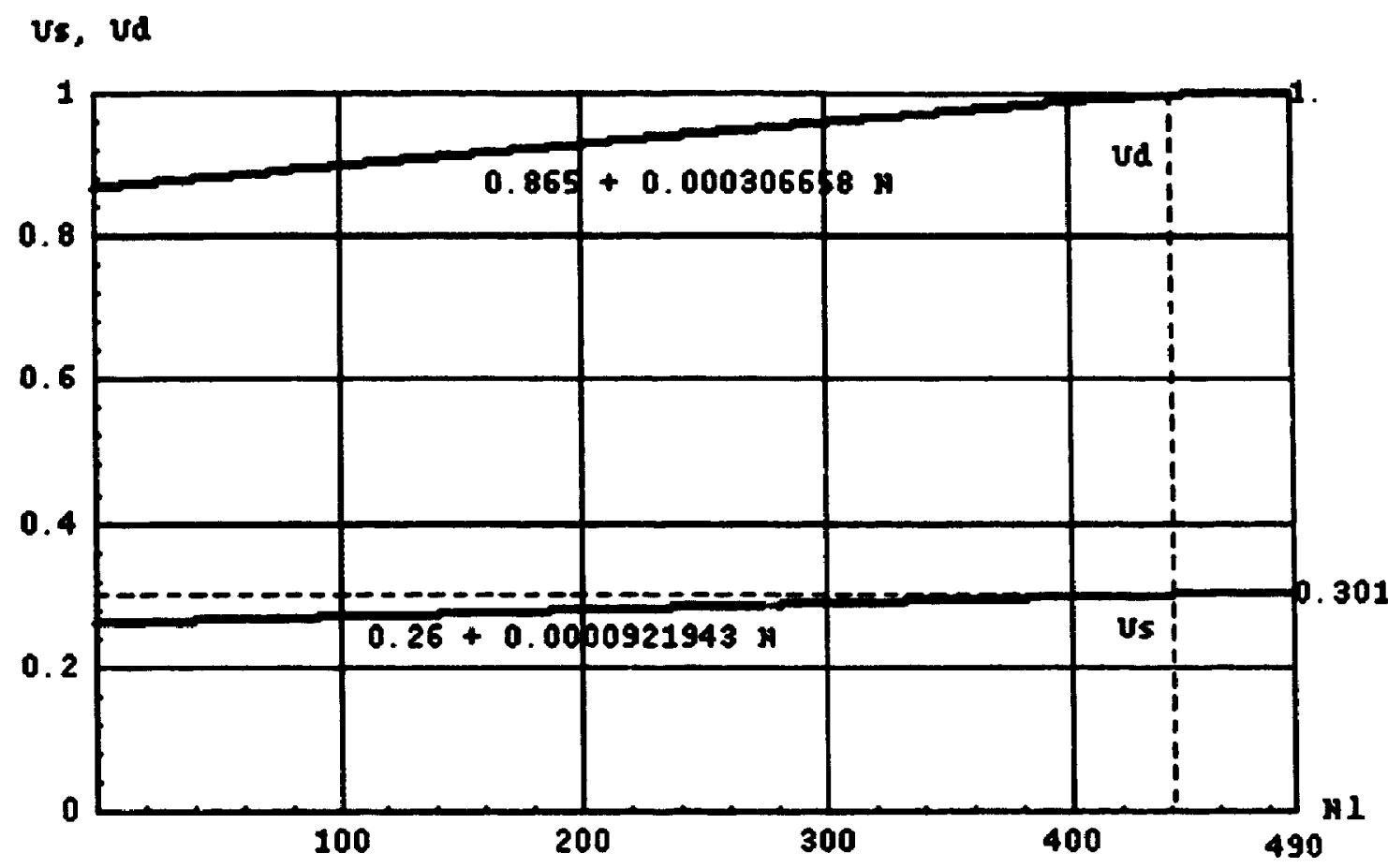

Figure D.2.4. Utilizations of the server Us and disk Ud, vs the number of local users NI with remote arrival rate 5 .

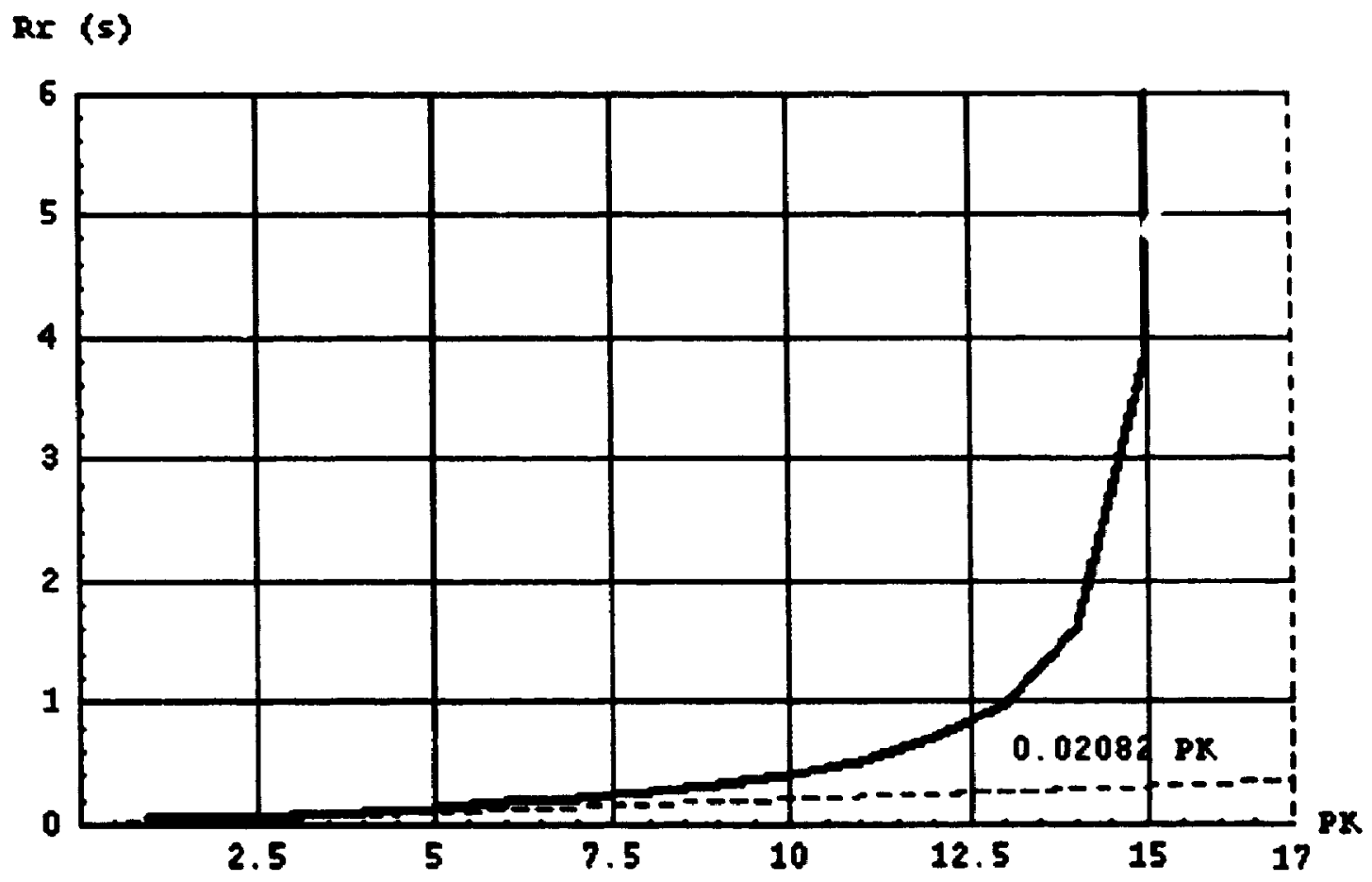

Figure D.3. Response time of remote requests $\operatorname{Rr}$ vs the number of packets of a request PK, with $\mathbf{2 8 0}$ local users and remote arrival rate 5 


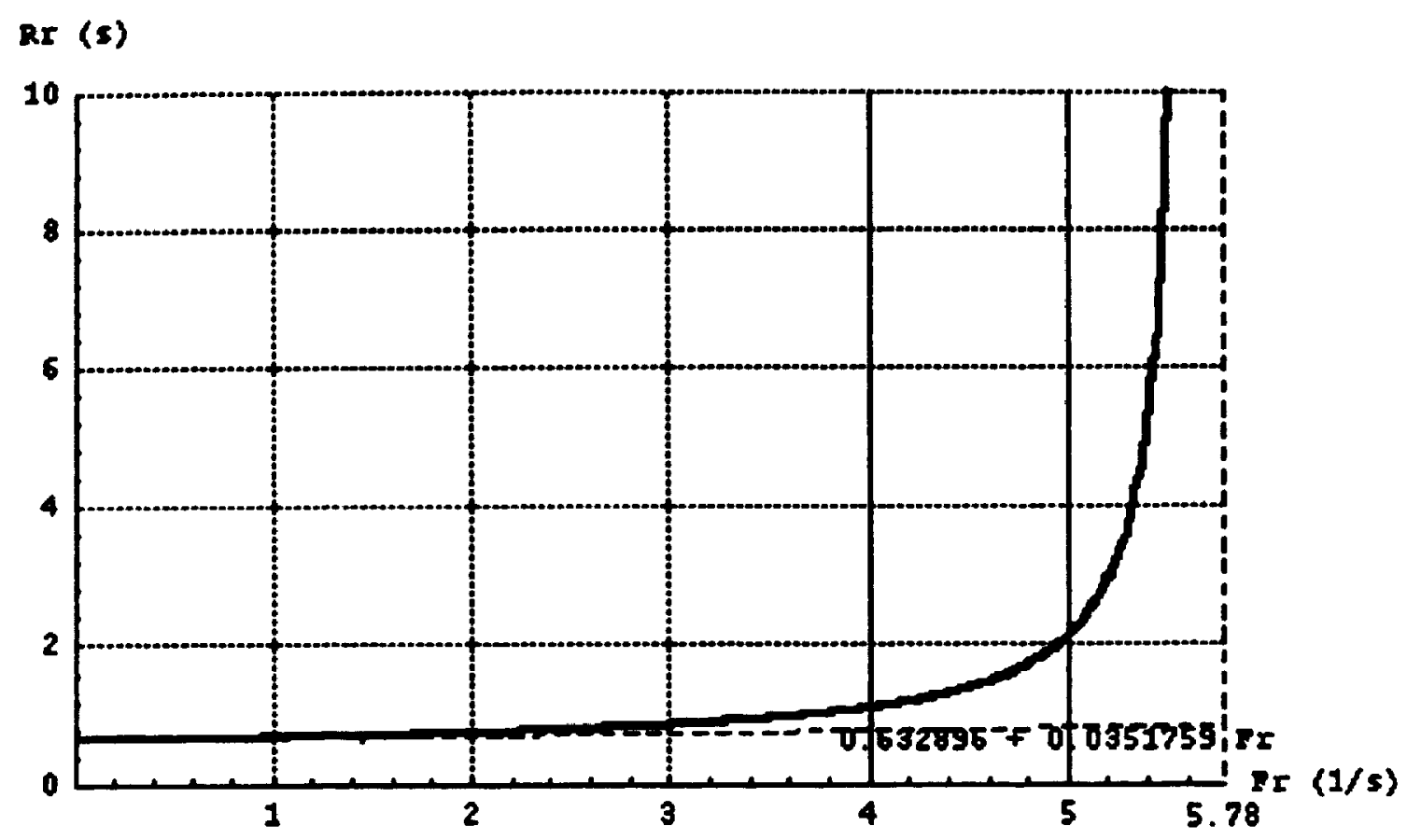

Figure D.4.1. Response time of remote request $R r$ vs remote arrival rate Fr, with 100 local users

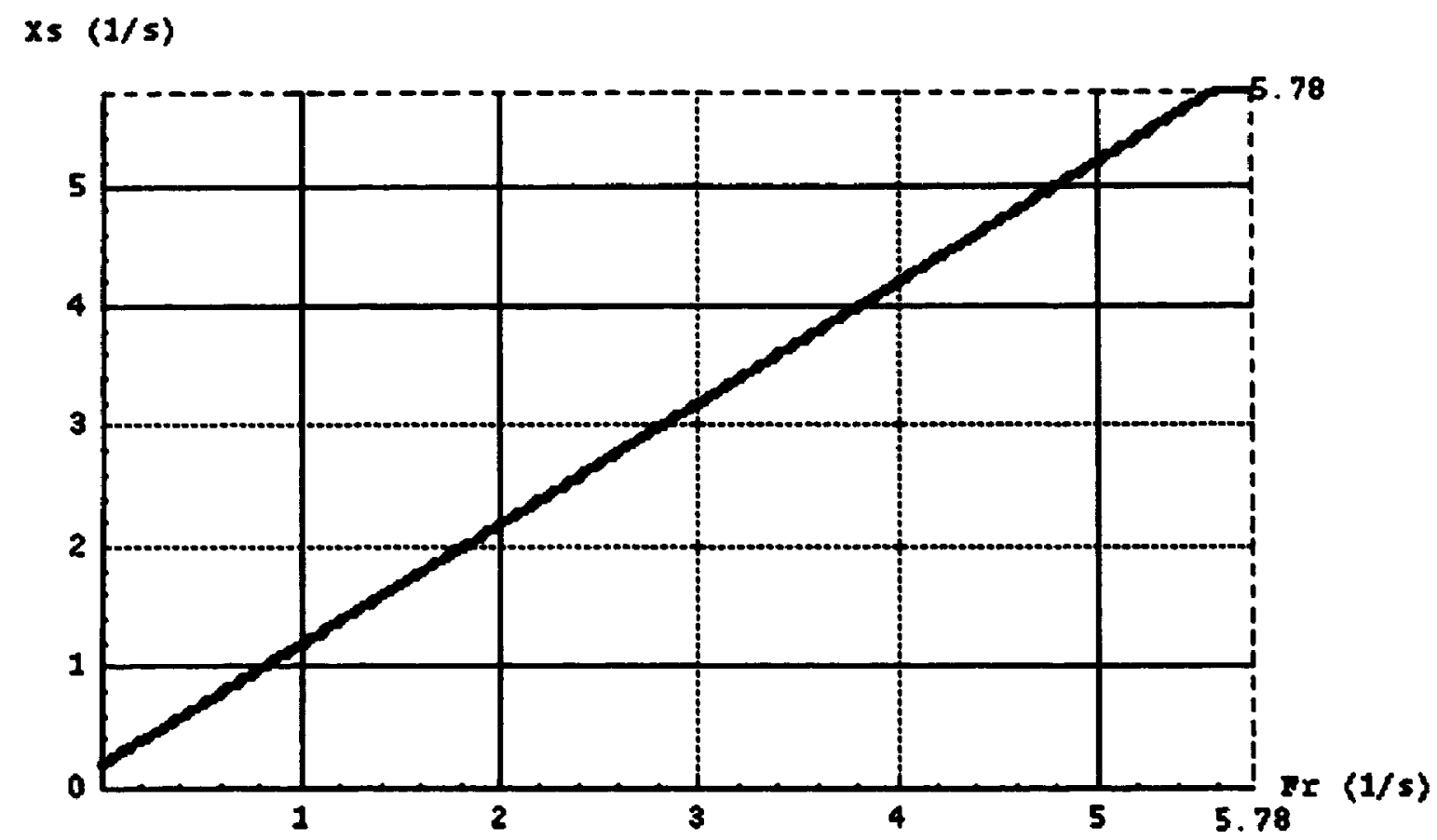

Figure D.4.2. Total request throughput $\mathrm{Xs}$ vs remote arrival rate Fr, with 100 local users. 
$x 1(1 / 8)$

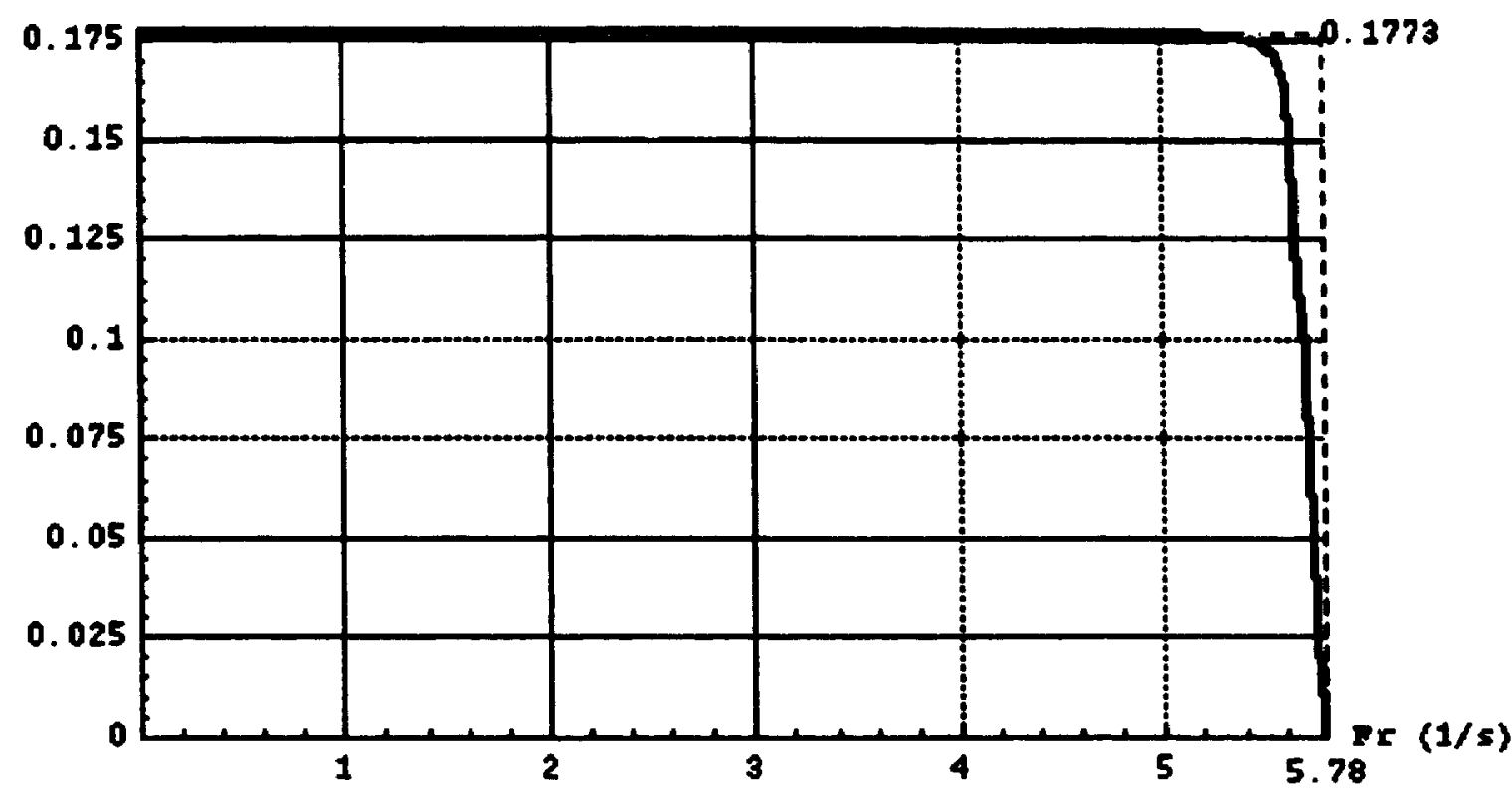

Figure D.4.3. Local request throughput $X 1$ vs remote arrival rate $F r$, with 100 local users.

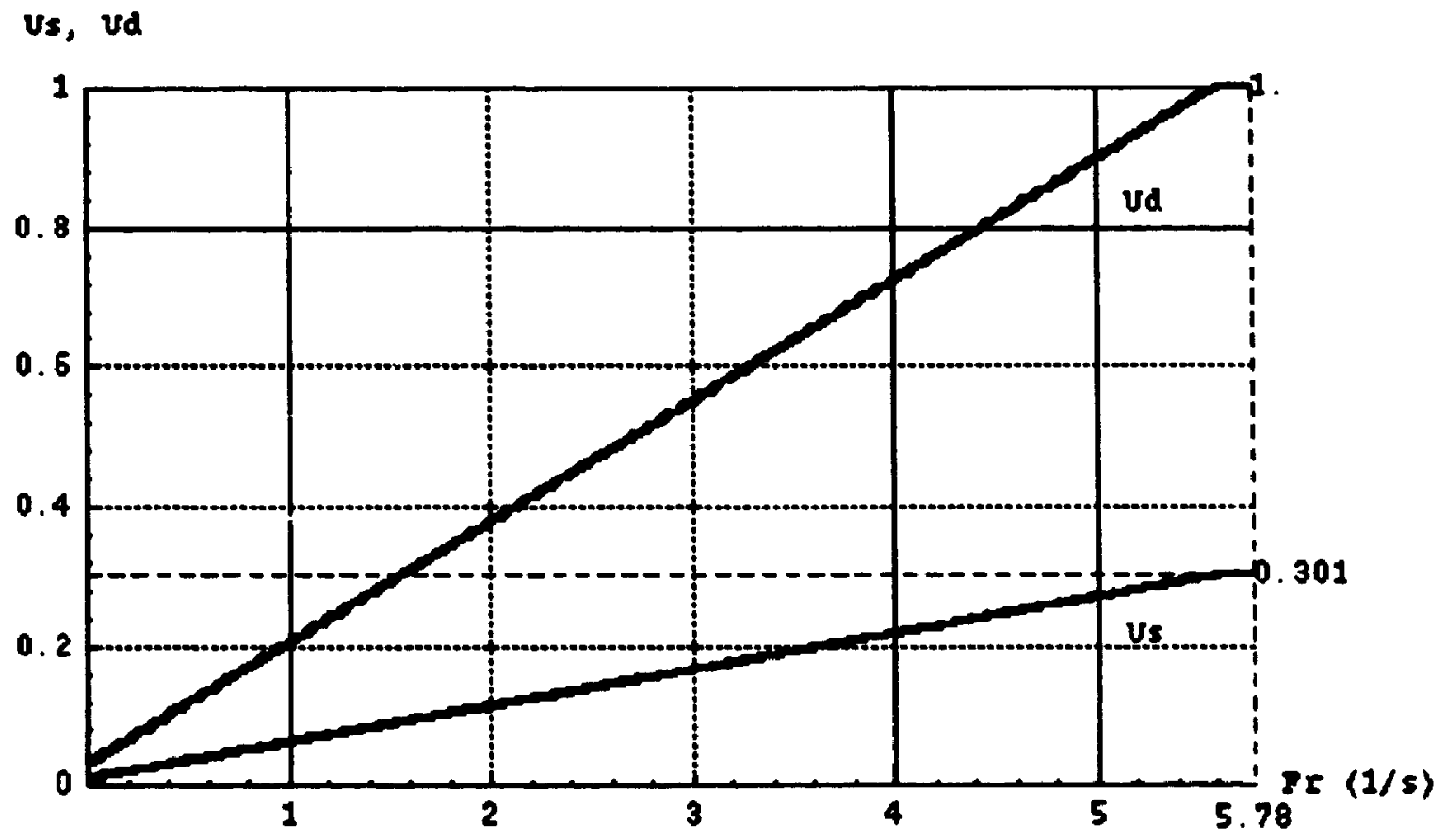

Figure D.4.4. Utilizations of the server Us and disk Ud, vs remote arrival rate $F r$, with 100 local users. 


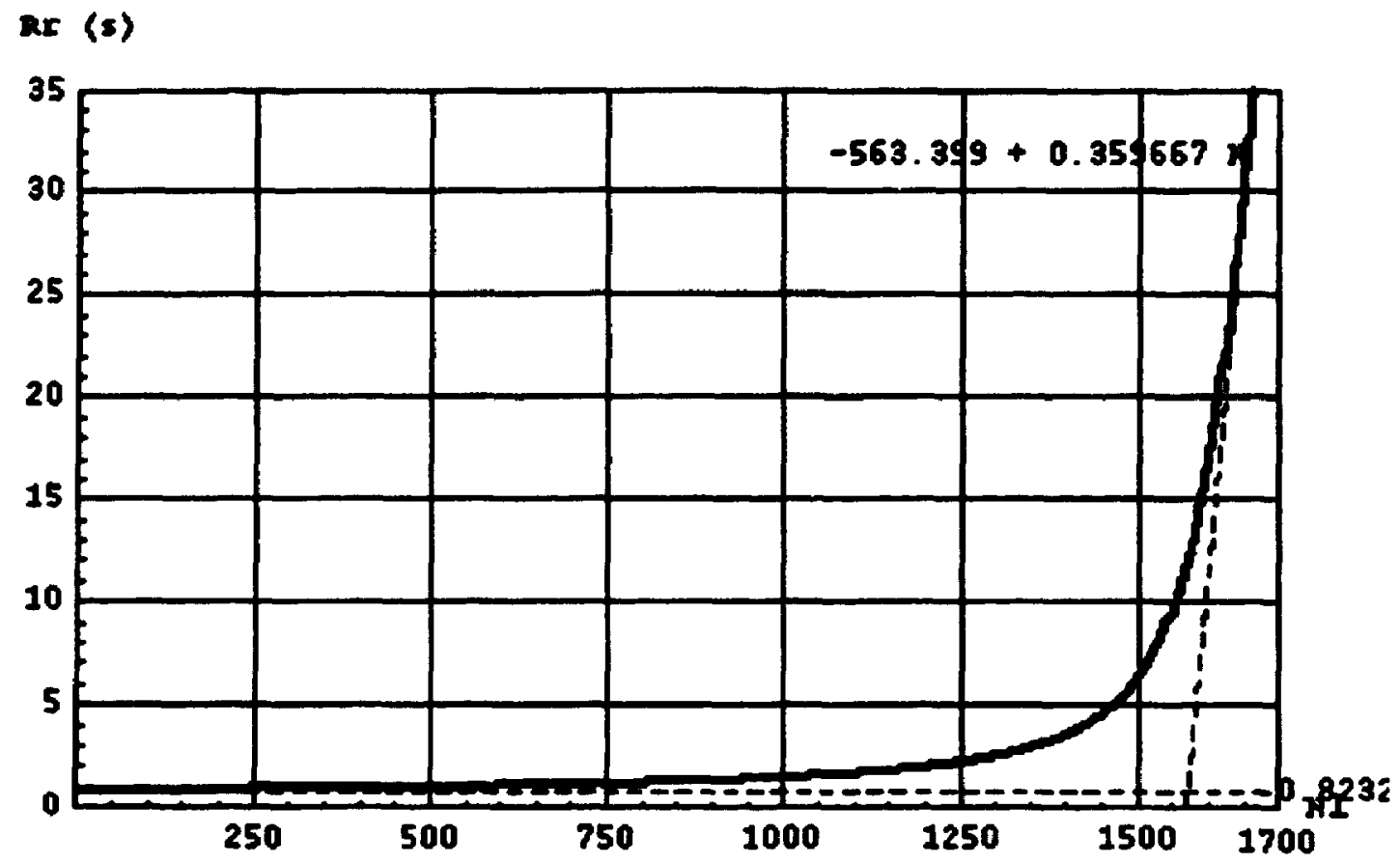

Figure D.5.1. Response time of remote requests Rr vs the number of local users NI, with remote arrival rate 3

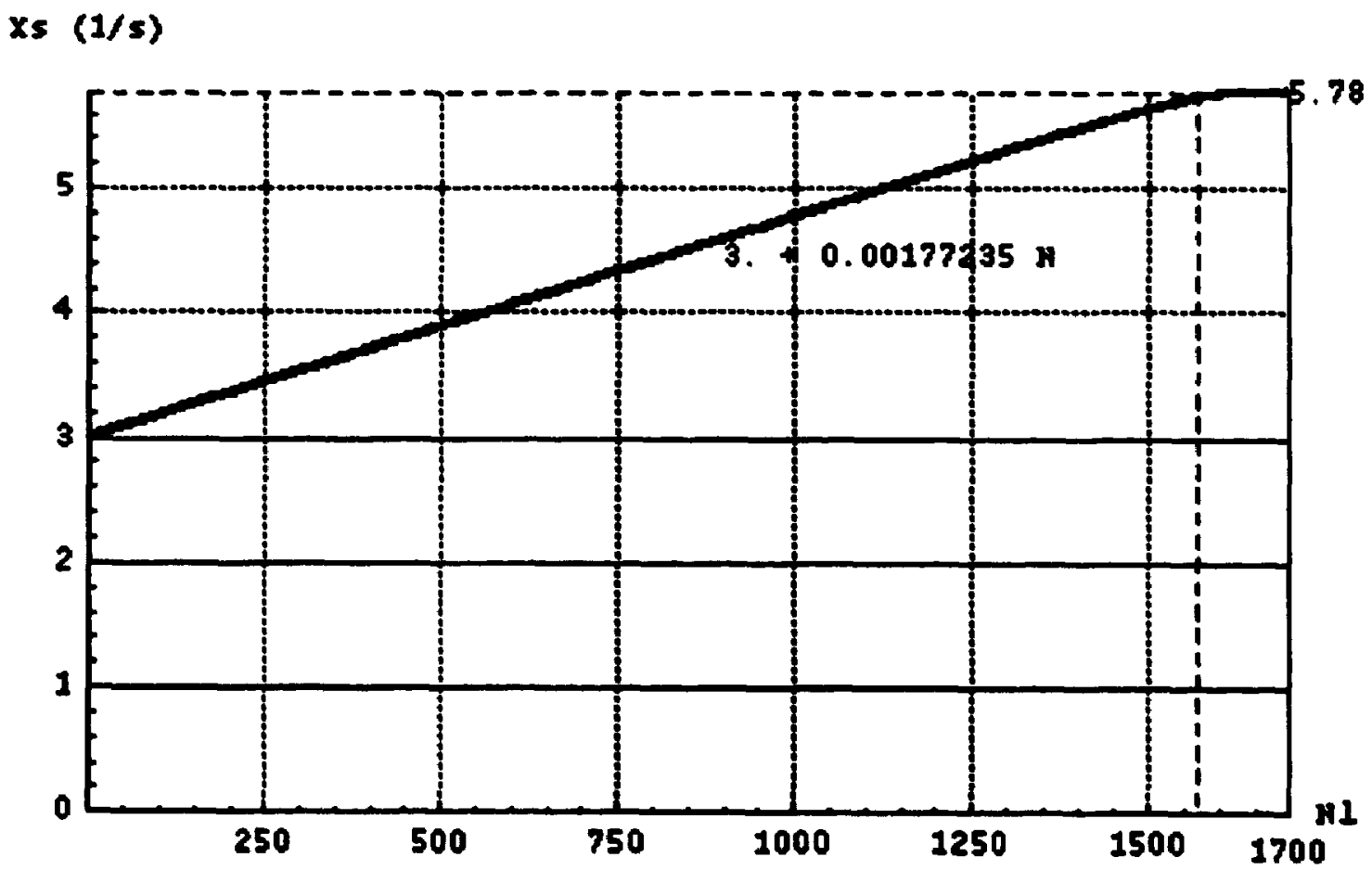

Figure D.5.2. Total request throughput $\mathrm{Xs}$ vs the number of local users $\mathrm{Nl}$, with remote arrival rate 3 
$x 1(1 / s)$

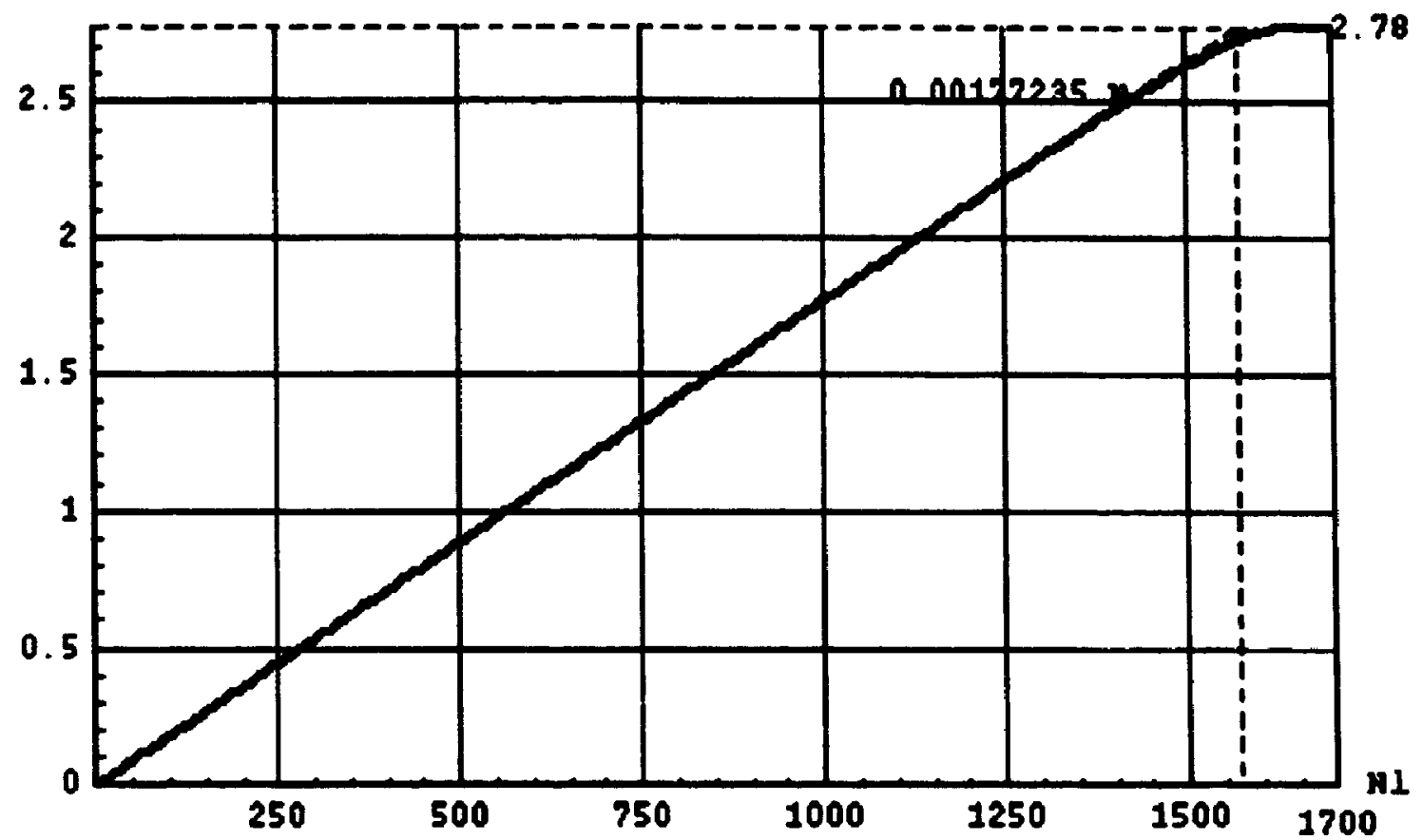

Figure D.5.3. Local request throughput $\mathrm{XI}$ vs the number of local users $\mathrm{NI}$, with remote arrival rate 3

Us, ud

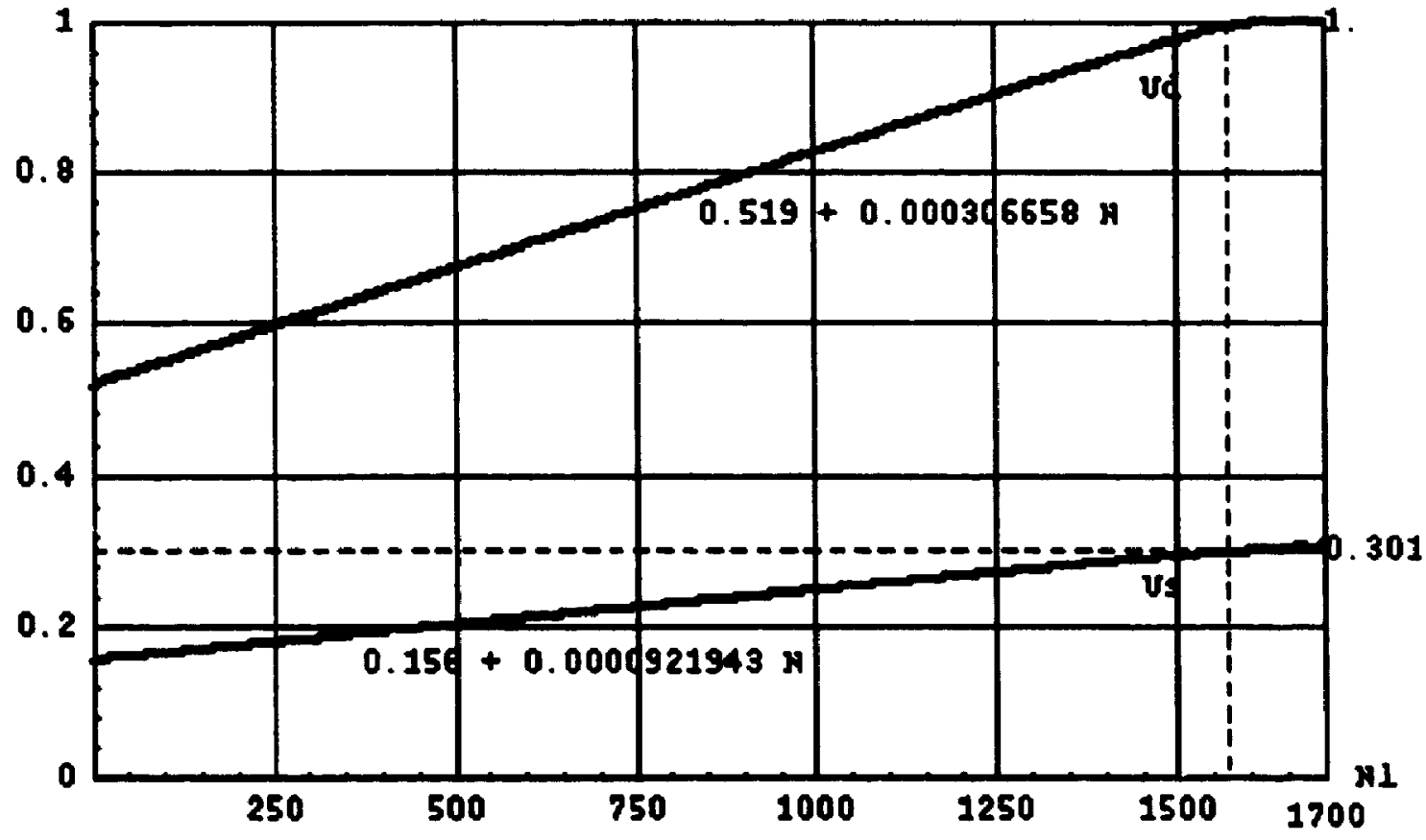

Figure D.5.4. Utilizations of the server Us and disk Ud, vs the number of local users $\mathrm{Nl}$ with remote arrival rate 3 


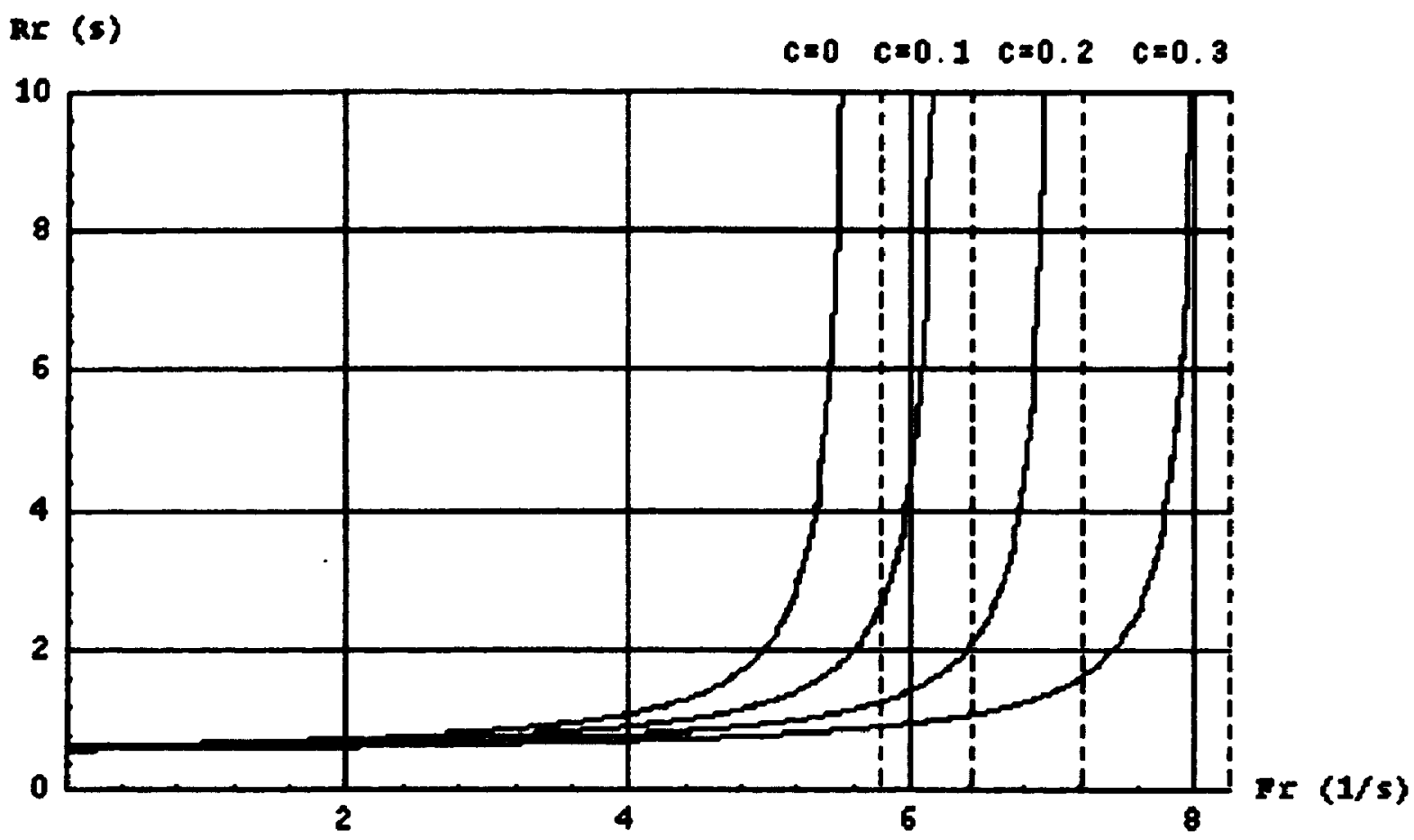

Figure D.6.1. Response time of remote request $\mathrm{Rr}$ vs remote arrival rate $\mathrm{Fr}$, with 100 local users and caching hit ratio $C=0,0.1,0.2,0.3$.

$x s(1 / 5)$

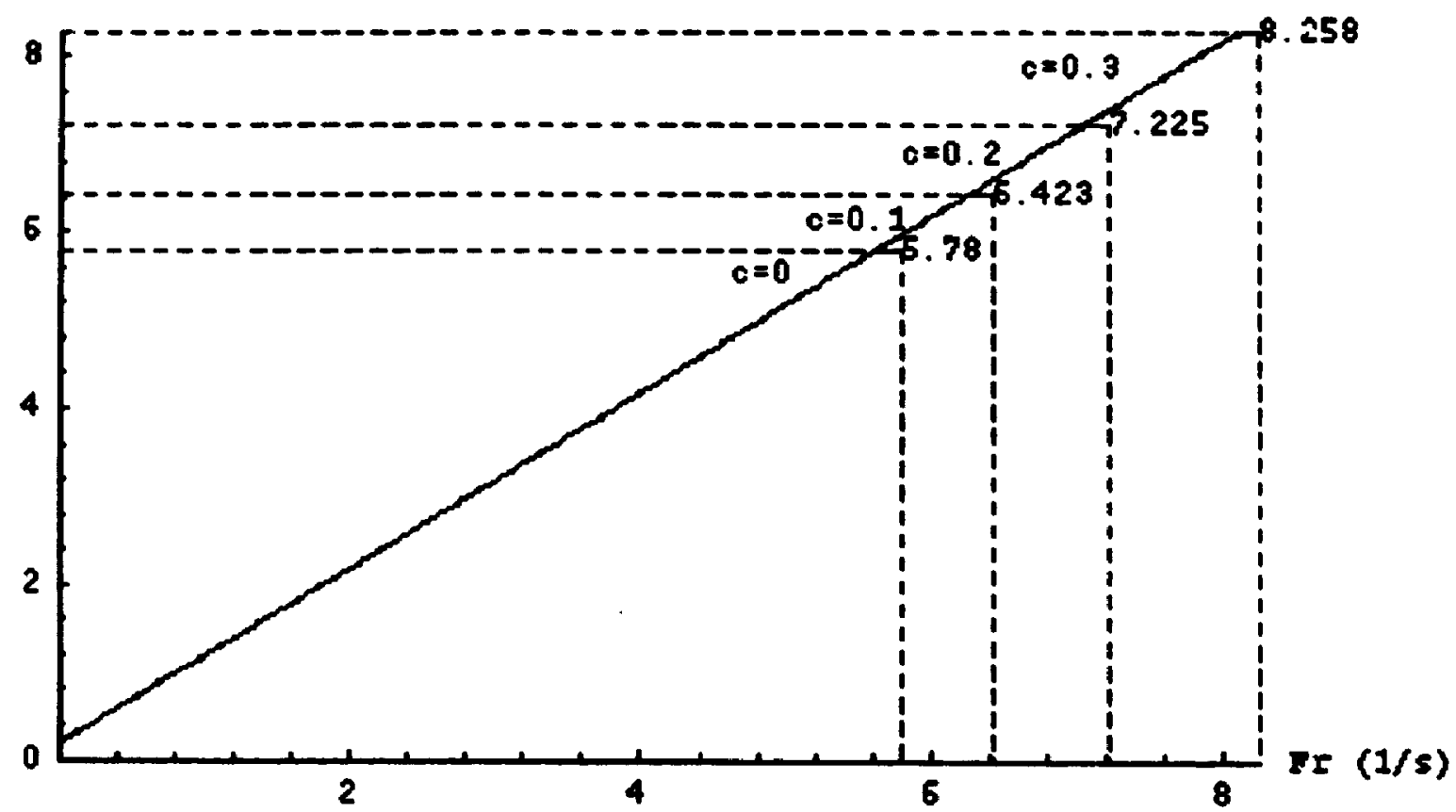

Figure D.6.2. Total request throughput $\mathrm{Xs}$ vs remote arrival rate $\mathrm{Fr}$, with 100 local users and caching hit ratio $C=0,0.1,0.2,0.3$. 
$x 1$ (1/s)

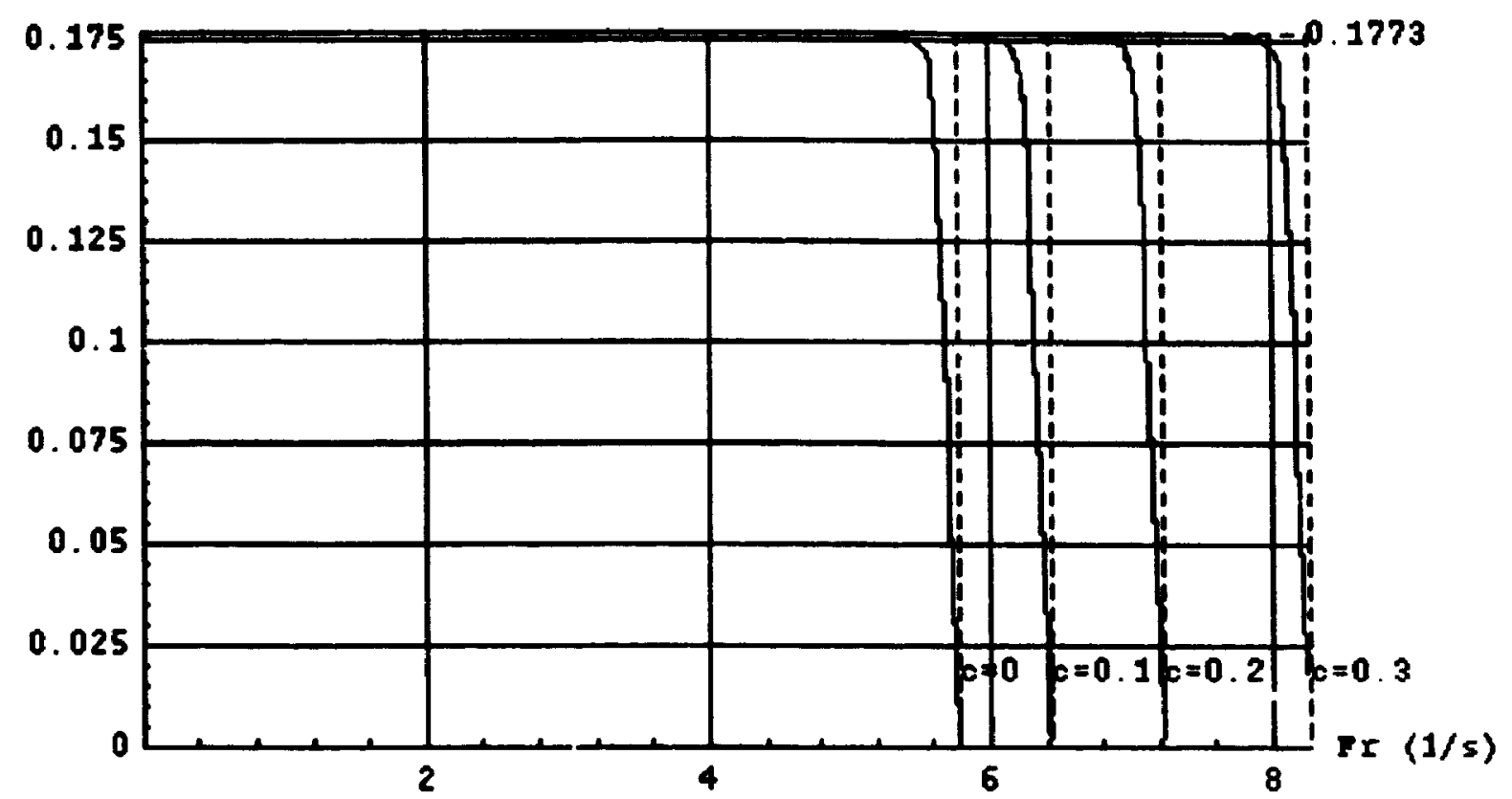

Figure D.6.3. Local request 'hroughput $\mathrm{XI}$ vs remote arrival rate $\mathrm{Fr}$, with 100 local users and caching hit ratio $C=0,0.1,0.2,0.3$.

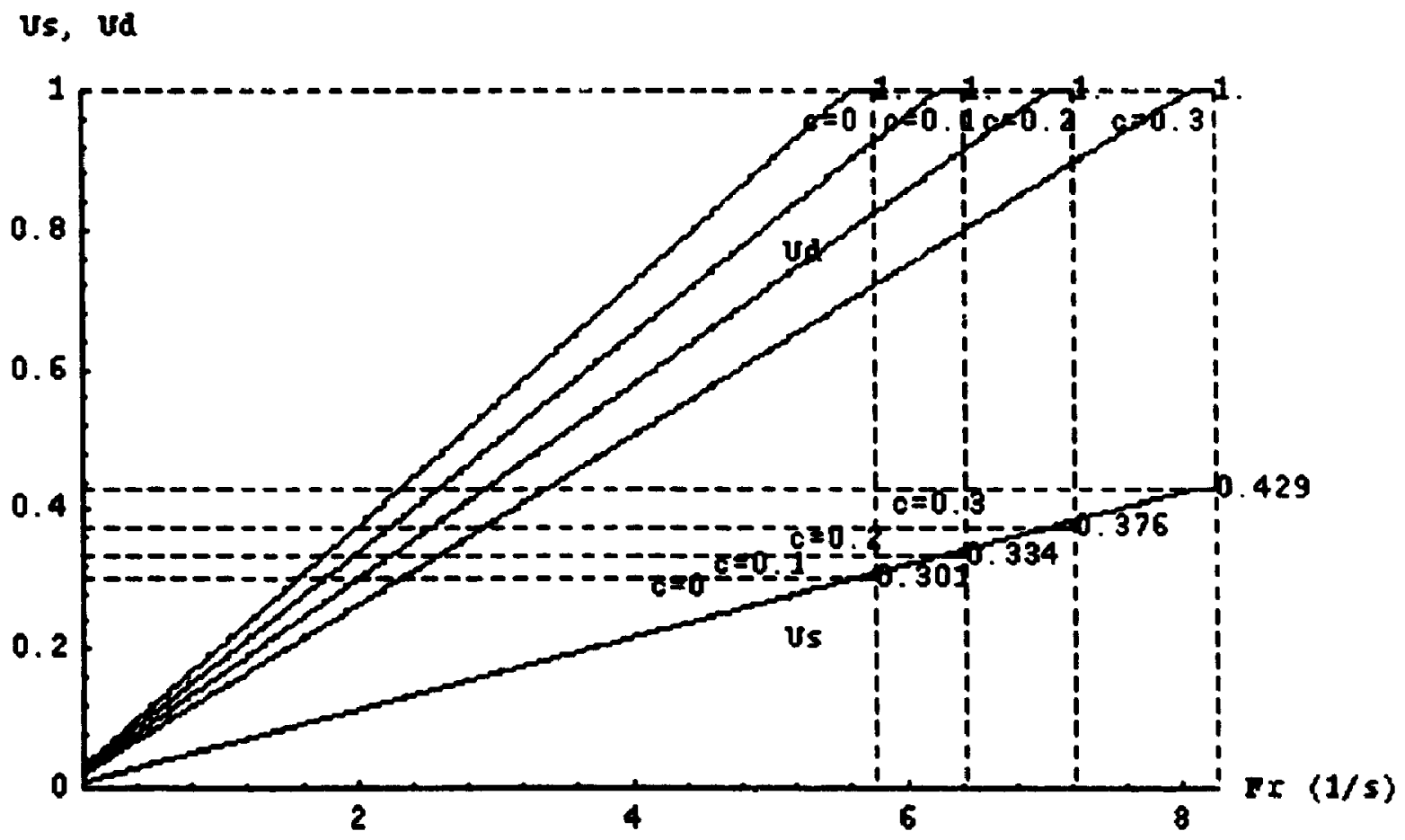

Figure D.6.4. Utilizations of the server Us and disk Ud, vs remote arrival rate Fr, with 100 local users and caching hit ratio $\mathrm{C}=0,0.1,0.2,0.3$. 

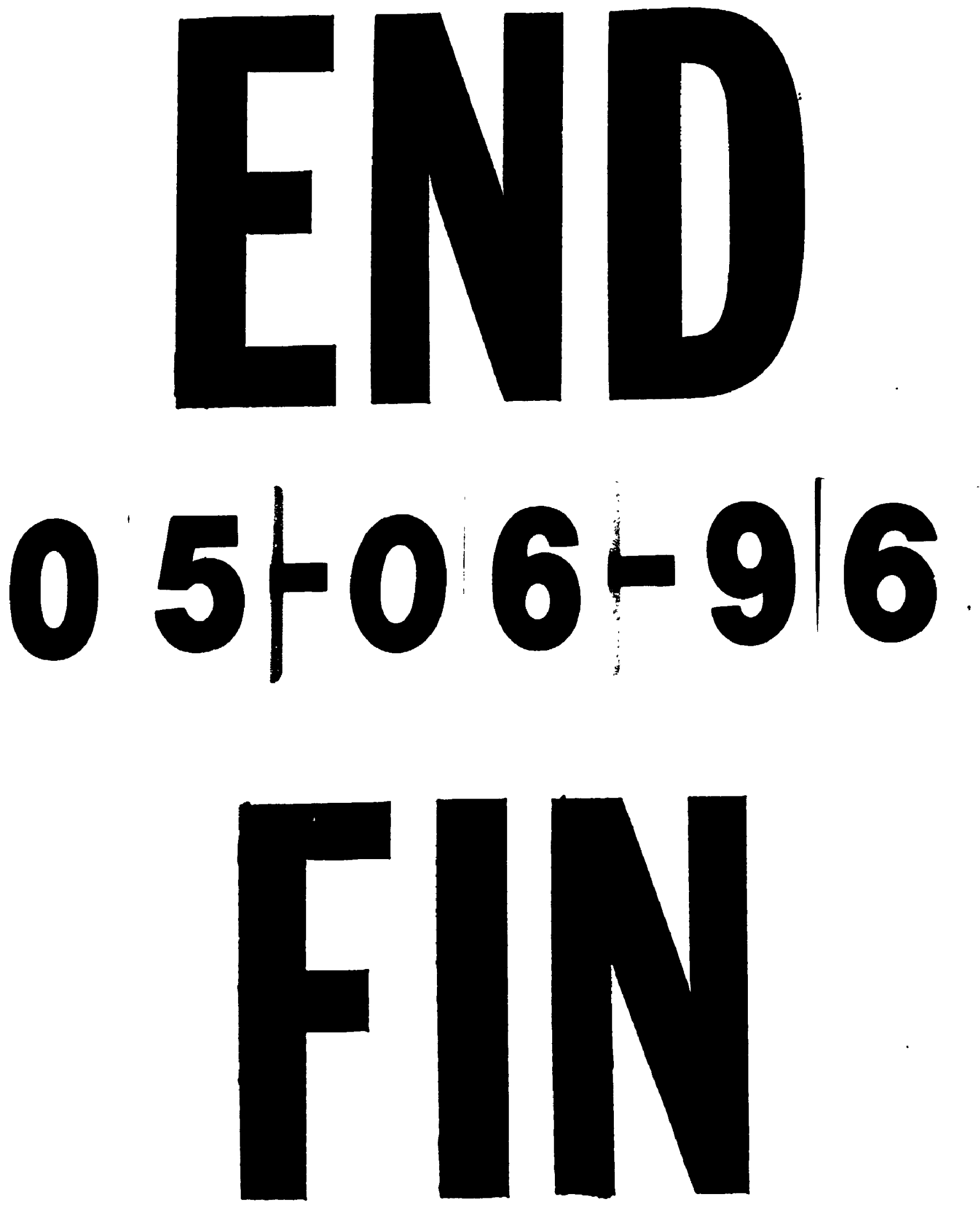